\title{
Ni-Catalyzed Enantioselective Intermolecular Hydroamination of Branched 1,3-Dienes Using Primary Aliphatic Amines
}

\author{
Gaël Tran, Wen Shao, Clément Mazet* \\ Department of Organic Chemistry, University of Geneva \\ Quai Ernest Ansermet, 30 - 1211 Geneva 4, Switzerland \\ clement.mazet@unige.ch
}

\section{Table of Contents}

1. General information

2. Reaction optimization

3. General procedure for the amination reaction

4. Substrate scope

4.1. Diene scope

4.2. Amine scope

5. Rh-catalyzed deallylation of 3ap

6. Improved sets of reaction conditions

7. Supporting organometallics

7.1. NMR monitoring

7.2. Synthesis of $\left[\left(L_{1}\right)_{2} \mathrm{Ni}\right](7)$

S72

7.3. Synthesis of $\left[\left(L_{1}\right) \mathrm{Ni}(\mathrm{cod})\right](8)$

$\mathrm{S} 72$

7.4. Synthesis of $\left[\left(L_{1}\right) \mathrm{Ni}(1 \mathrm{a})\right](9)$

S74

7.5. Synthesis of $\left[\left(L_{1}\right) \mathrm{Ni}\left(\eta^{3}-\mathrm{Allyl}\right)\right]\left[\mathrm{OCH}_{2} \mathrm{CF}_{3}\right](10)$

S75

7.6. Synthesis of $\left[\left(L_{1}\right) \mathrm{Ni}(3 \mathrm{aa})\right](11)$

S76

7.7. Reaction of $\left[\left(\mathrm{L}_{1}\right) \mathrm{Ni}\left(\eta^{3}\right.\right.$-allyl $\left.)\right]\left[\mathrm{OCH}_{2} \mathrm{CF}_{3}\right](10)$ with $\mathrm{BnNH}_{2}$

S77

8. Amine exchange experiment

S80

9. Deuterium labeling experiments

9.1. Model catalytic hydroamination using TFE- $d_{1}$ and $B_{n N D_{2}}$

S81

9.2. Post-reaction deuterium incorporation experiment

S84

9.3. Synthesis of 10- $d_{n}$ using TFE- $d_{1}-{ }^{2} H$ NMR monitoring

$\mathrm{S} 85$

9.4. Synthesis of $10-d_{n}$ using TFE- $d_{1}$ and $B n N D_{2-}{ }^{1} \mathrm{H}$ NMR monitoring

S86

10. Kinetic study

11. $\mathrm{BnNH}_{2}$ NMR titration by TFE

12. NMR Spectra of organic compounds

13. NMR Spectra or organometallic compounds 


\section{General Information}

Unless otherwise noted, all reactions were carried out under air. All liquid amines were distilled and degassed by three successive "freeze-pump-thaw" cycles, followed by drying over activated $4 \AA \mathrm{MS}$. THF, $\mathrm{Et}_{2} \mathrm{O} \mathrm{CH}_{2} \mathrm{Cl}_{2}$, toluene, and pentane were degassed by $\mathrm{N}_{2}$ bubbling and dried over activated alumina columns. Mesitylene was dried and distilled over $\mathrm{CaH}_{2}$ and degassed by three successive "freeze-pump-thaw" cycles. $\mathrm{Ni}(\operatorname{cod})_{2}$ was dissolved in toluene (ca. $1 \mathrm{~g} / 20 \mathrm{~mL})$, filtered over Celite, and recrystallized at $-78{ }^{\circ} \mathrm{C}$. Unless otherwise specified, all the dienes were prepared according to reported literature procedures. ${ }^{1}$ All other reagents were purchased from Aldrich, Fluka, Acros or Strem and used without purification. NMR spectra were recorded on AMX-400 and AMX-500 Bruker Avance spectrometers at $298 \mathrm{~K} .{ }^{1} \mathrm{H}$ and ${ }^{13} \mathrm{C}\left\{{ }^{1} \mathrm{H}\right\} \mathrm{NMR}$ chemical shifts are given in ppm relative to $\mathrm{SiMe}_{4}$, with the solvent resonance used as internal reference. ${ }^{1} \mathrm{H} \mathrm{NMR}$ spectra were referenced to $\mathrm{CHCl}_{3}(\delta=$ $7.26 \mathrm{ppm})$, methanol $(\delta=3.31 \mathrm{ppm})$ and toluene $(\delta=6.98 \mathrm{ppm})$ and ${ }^{13} \mathrm{C}\left\{{ }^{1} \mathrm{H}\right\}$ NMR spectra were referenced to $\mathrm{CDCl}_{3}(\delta=77.16 \mathrm{ppm})$, methanol- $d_{4}(\delta=49.00 \mathrm{ppm})$ and toluene- $d_{8}(\delta=$ $20.40 \mathrm{ppm}) \cdot{ }^{31} \mathrm{P}\left\{{ }^{1} \mathrm{H}\right\} \mathrm{NMR}$ chemical shifts are reported in ppm relative to $\mathrm{H}_{3} \mathrm{PO}_{4} \cdot{ }^{19} \mathrm{~F}\left\{{ }^{1} \mathrm{H}\right\} \mathrm{NMR}$ chemical shifts are reported in ppm relative to $\mathrm{CFCl}_{3}$. Infrared spectra were obtained on a Perkin-Elmer 1650 FT-IR spectrometer using neat samples on a diamond ATR Golden Gate sampler. HRMS were obtained on a Xevo G2 Tof spectrometer (Ionization mode: ESI positive polarity; Mobile phase: $\mathrm{MeOH} 100 \mu \mathrm{l} / \mathrm{min}$ ). Mass spectrum is calibrated by the use of the MS lockspray system (LeuEnk calibration solution). The enantiomeric excesses (ee's) were determined by HPLC, SFC and GC analyses. HPLC analyses were performed on a Shimadzu CTO-20AA with column DAICEL OD-H, OJ-H, AD-H and IC. SFC analyses were performed on a Waters Acquity UPC2 with columns OD-3, OJ-3, OZ-3, OB-H, AZ-3, AD, AS$3, A Y-H$. Retention times $\left(t_{R}\right)$ are given in minutes. GC analyses were performed on HP6890, column HYDRODEX $\gamma$-DiMOM, HYDRODEX $\beta$-3P and HYDRODEX TBDM, $50 \mathrm{~m}$. Optical rotations were recorded using an OMNI Lab JASCO P-1030 polarimeter using 589 $\mathrm{nm}$ emission band of a sodium lamp. Thin layer chromatography (TLC) was performed on plates of silica precoated with $0.25 \mathrm{~mm}$ Kieselgel $60 \mathrm{~F}_{254}$ from Merck. Flash chromatography was performed using silica gel SiliaFlash ${ }^{8}$ P60 (230-400 mesh) from Silicycle. 


\section{Reaction optimization}
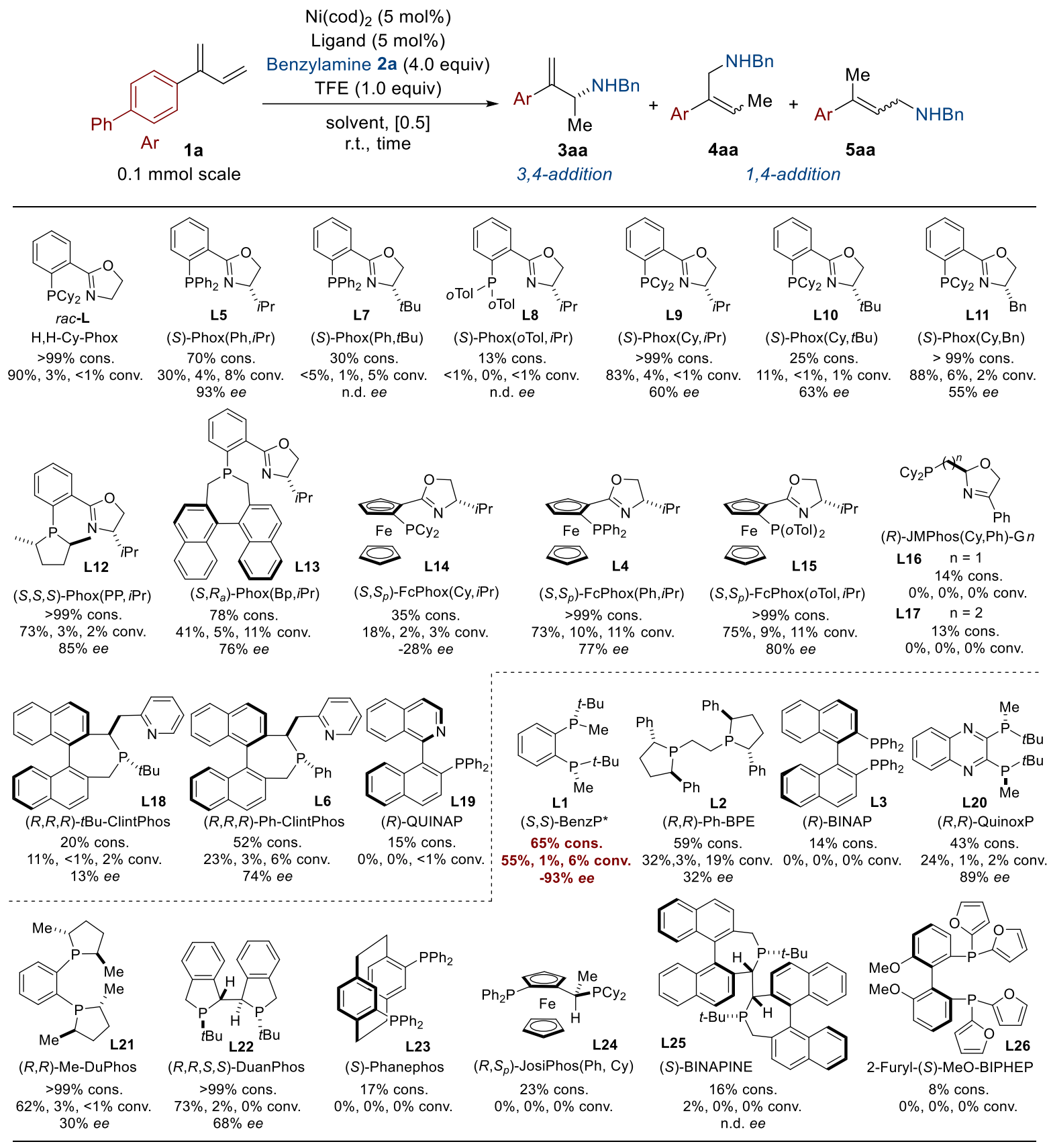

Table S1: Ligand screening. ${ }^{a}$ Reaction conditions: all reactions were performed with diene 1a $\left(0.10 \mathrm{mmol}, 1.0\right.$ equiv), benzylamine 2a $\left(0.40 \mathrm{mmol}, 4.0\right.$ equiv), $\mathrm{Ni}(\mathrm{cod})_{2}(0.005 \mathrm{mmol}, 5$ mol\%), ligand (0.005 mmol, $5 \mathrm{~mol} \%)$, 2,2,2-trifluoroethanol (0.10 mmol, 1.0 equiv) in mesitylene $(0.20 \mathrm{~mL}, 0.5 \mathrm{M})$ at room temperature. Consumption of 1a, conversions of 3aa, 4aa and 5aa were assessed by ${ }^{1} \mathrm{H}$ NMR of the crude reaction mixture using an internal standard. n.d. $=$ not determined. 


\begin{tabular}{|c|c|c|c|c|c|c|c|c|c|c|}
\hline $0.1 \mathrm{~m}$ & $\begin{array}{l}\text { 1a } \\
\text { mol scale }\end{array}$ & $\begin{array}{r}\mathrm{Ni}(\operatorname{cod})_{2}(5 \mathrm{~mol} \\
(R, R) \text {-BenzP L1 }(5 \mathrm{n} \\
\text { Benzylamine } 2 \mathrm{a}(\mathrm{x} \\
\text { Additive (y equi } \\
\begin{array}{c}\text { solvent, [0.5] } \\
\text { r.t., time }\end{array}\end{array}$ & $\begin{array}{l}\text { ol\%) } \\
\text { uiv) } \\
\longrightarrow\end{array}$ & $\begin{array}{l}3 \mathbf{a a} \\
- \text {-addit }\end{array}$ & & $\int_{4}^{\mathrm{NHBn}} \mathrm{Me}+$ & $5 \mathbf{a}$ & & $\begin{array}{r}\mathrm{L}_{1} \\
(R, R)-\end{array}$ & enz \\
\hline Entry & Solvent & Additive & $\begin{array}{c}\text { Time } \\
\text { (h) }\end{array}$ & $x$ & $y$ & $\begin{array}{c}\text { 1a Cons. } \\
(\%)\end{array}$ & $\begin{array}{l}\text { 3aa } \\
(\%)\end{array}$ & $\begin{array}{l}\text { 4aa } \\
(\%)\end{array}$ & $\begin{array}{l}\text { 5aa } \\
(\%)\end{array}$ & $\begin{array}{c}\text { ee 3aa } \\
(\%)\end{array}$ \\
\hline $1^{b}$ & Mes. & TFE & 24 & 4 & 1 & 65 & 55 & 1 & 6 & -93 \\
\hline $2^{b}$ & Mes. & TFE & 48 & 4 & 1 & $>99$ & 72 & 2 & 8 & -93 \\
\hline 3 & PhMe & TFE & 24 & 4 & 1 & 60 & 52 & $<1$ & 8 & 91 \\
\hline 4 & Mes. & TFE & 48 & 4 & 1 & $>99$ & 79 & 3 & 11 & 93 \\
\hline 5 & THF & TFE & 48 & 4 & 1 & 57 & 43 & 2 & 4 & 95 \\
\hline 6 & $\mathrm{Et}_{2} \mathrm{O}$ & TFE & 48 & 4 & 1 & 46 & 36 & 1 & 3 & 95 \\
\hline 7 & $\mathrm{Et}_{2} \mathrm{O}$ & TFE & 48 & 4 & 1 & $>99$ & 74 & 2 & 8 & 93 \\
\hline 8 & $\mathrm{CH}_{2} \mathrm{Cl}_{2}$ & TFE & 48 & 4 & 1 & 8 & 0 & 0 & 0 & -- \\
\hline 9 & Mes. & TFE & 48 & 2 & 1 & 30 & 17 & $<1$ & 2 & 86 \\
\hline $10^{c}$ & Mes. & TFE & 24 & 4 & 1 & $>99$ & 70 & 3 & 11 & 87 \\
\hline 11 & Mes & $\mathrm{EtOH}$ & 24 & 4 & 1 & 4 & 0 & 0 & 0 & -- \\
\hline 12 & Mes. & $\mathrm{Ph}_{2} \mathrm{P}(\mathrm{O}) \mathrm{OH}$ & 24 & 4 & 1 & $>99$ & 88 & 3 & 4 & 90 \\
\hline 13 & $\mathrm{Et}_{2} \mathrm{O}$ & $\mathrm{Ph}_{2} \mathrm{P}(\mathrm{O}) \mathrm{OH}$ & 24 & 4 & 1 & 80 & 72 & 2 & 2 & 83 \\
\hline 14 & Mes. & $\mathrm{Ph}_{2} \mathrm{P}(\mathrm{O}) \mathrm{OH}$ & 48 & 2 & 1 & $>99$ & 84 & 3 & 3 & 88 \\
\hline 15 & Mes. & $\mathrm{Ph}_{2} \mathrm{P}(\mathrm{O}) \mathrm{OH}$ & 48 & 4 & 0.2 & $>99$ & 92 & 3 & 4 & 91 \\
\hline 16 & Mes. & $\mathrm{Ph}_{2} \mathrm{P}(\mathrm{O}) \mathrm{OH}$ & 48 & 2 & 0.2 & $>99$ & 90 & 3 & 2 & 91 \\
\hline
\end{tabular}

Table S2: Other condition optimization. ${ }^{a}$ Reaction conditions: all reactions were performed with diene $1 \mathrm{a}(0.10 \mathrm{mmol}, 1.0$ equiv), benzylamine $2 \mathrm{a}(0.40 \mathrm{mmol}, 4.0$ equiv or $0.20 \mathrm{mmol}, 2.0$ equiv), Ni(cod) $)_{2}(0.005 \mathrm{mmol}, 5 \mathrm{~mol} \%),(R, R)-$ BenzP* $^{*}$ L1 $(0.005 \mathrm{mmol}, 5$ mol\%), 2,2,2-trifluoroethanol $(0.10 \mathrm{mmol}, 1.0$ equiv) or diphenylphosphonic acid $(0.10 \mathrm{mmol}$, 1.0 equiv or $0.020 \mathrm{mmol}, 0.20$ equiv) in solvent $(0.20 \mathrm{~mL}, 0.5 \mathrm{M})$ at room temperature. Consumption of $\mathbf{1 a}$ and conversions were assessed by ${ }^{1} \mathrm{H}$ NMR of the crude reaction mixture using an internal standard. ${ }^{b}$ Using $(S, S)-$ BenzP $^{*}$ as ligand. ${ }^{c}$ At $40{ }^{\circ} \mathrm{C}$. Mes. = mesitylene, $\mathrm{THF}=$ tetrahydrofuran, TFE $=$ 2,2,2-trifluoroethanol. 


\section{General procedure for the amination reaction}

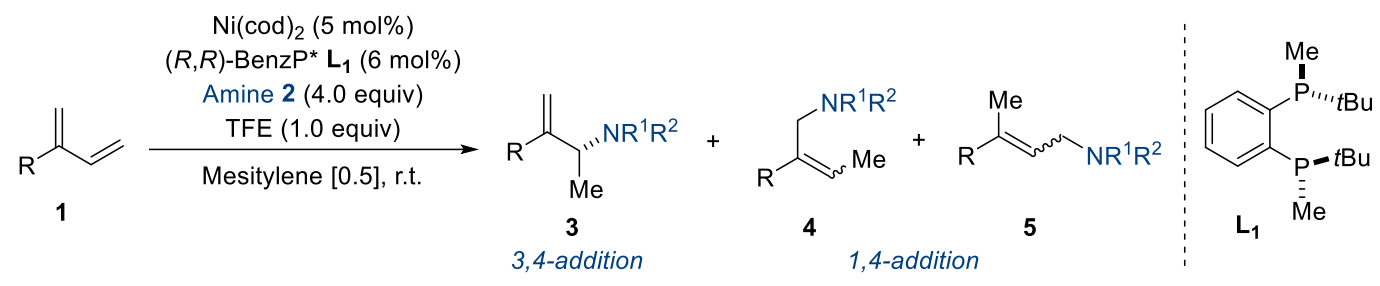

In a $\mathrm{N}_{2}$-filled glovebox, $\mathrm{Ni}(\mathrm{cod})_{2}(0.0125 \mathrm{mmol}, 3.4 \mathrm{mg}, 5 \mathrm{~mol} \%)$ and $(R, R)$-BenzP* $\mathbf{L}_{\mathbf{1}}(0.015$ $\mathrm{mmol}, 4.2 \mathrm{mg}, 6 \mathrm{~mol} \%$ ) were charged in a $5 \mathrm{~mL}$ Schlenk tube and dissolved in anhydrous mesitylene $(0.50 \mathrm{~mL}, 0.50 \mathrm{M})$. After stirring at room temperature for $10 \mathrm{~min}$, the appropriate diene 1 (0.25 mmol, 1.0 equiv), the appropriate amine 2 (1.00 mmol, 4.0 equiv) and TFE $(0.25 \mathrm{mmol}, 19 \mu \mathrm{L}, 1.0$ equiv) were added sequentially. The tube was sealed, taken out of the glovebox and the reaction mixture was stirred at room temperature. After complete consumption of diene $\mathbf{1}$ (determined by TLC), the reaction mixture was filtered over a short pad of silica gel, washed with ethyl acetate $(10 \mathrm{~mL})$ and concentrated under vacuum to afford the crude mixture. The conversion and isomeric ratio were determined by ${ }^{1} \mathrm{H}$ NMR analysis of the crude reaction mixture using $\mathrm{CH}_{2} \mathrm{Br}_{2}$ as an internal standard. The residue was purified by silica gel column chromatography to afford the analytically pure hydroamination product 3 .

Note: All racemates were prepared according to a similar procedure using $\mathrm{H}, \mathrm{H}-\mathrm{Cy}$-Phox rac- $L$ as ligand (mesitylene [0.5], room temperature, $24 \mathrm{~h}$ ). 


\section{Substrate scope}

\subsection{Scope in diene}
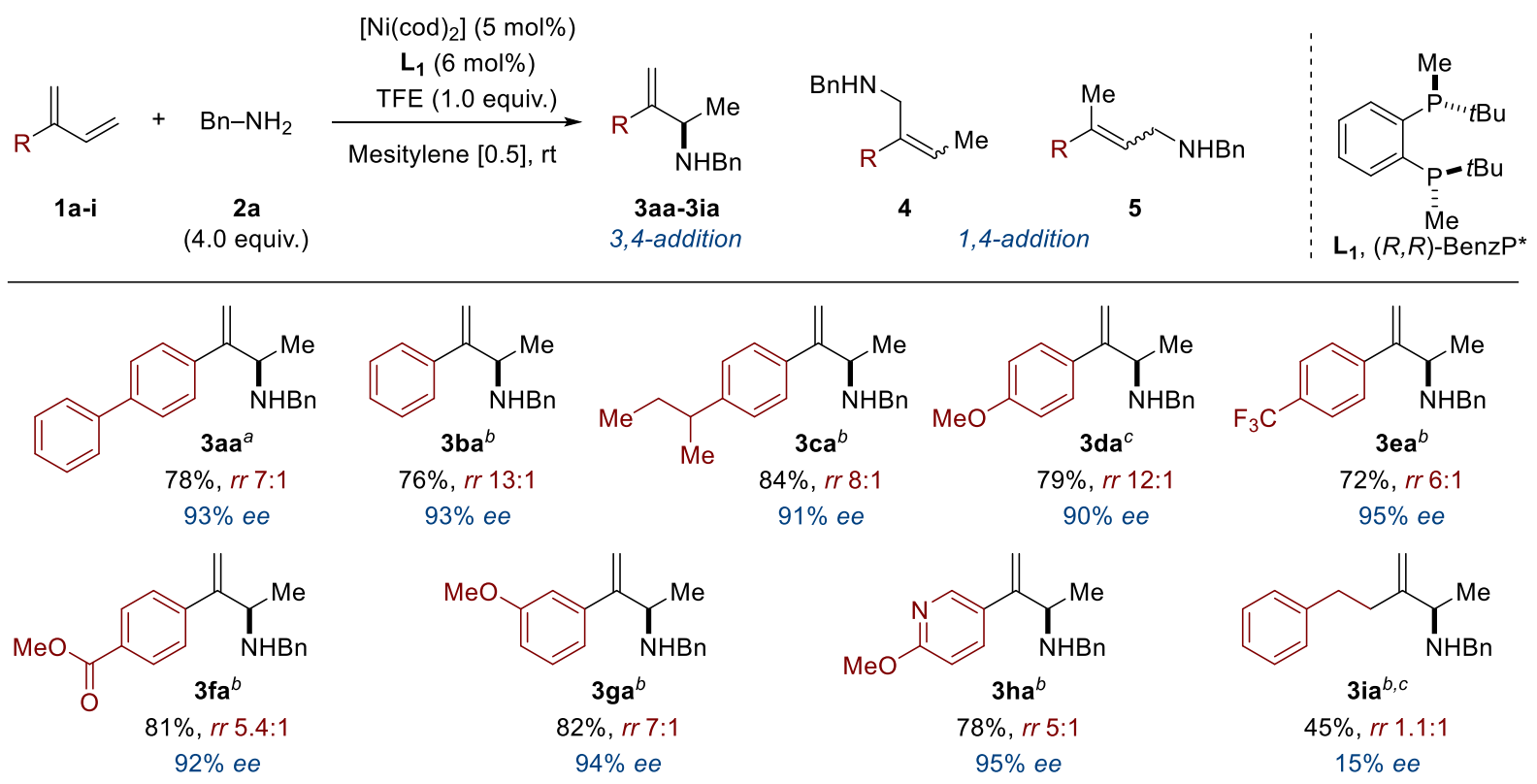

${ }^{a} 48$ h. ${ }^{b} 72$ h. ${ }^{c} 120$ h. ${ }^{d} \mathrm{Ph}_{2} \mathrm{P}(\mathrm{O}) \mathrm{OH}$ instead of TFE

Figure S1. Scope of the Ni-catalyzed hydroamination of 2-substituted 1,3-dienes Variation of the diene component. $0.25-0.40 \mathrm{mmol}$ scale. Yields of 3,4-addition product after purification. Regioselectivity expressed as the ratio between 3,4- and 1,4-addition products as determined by ${ }^{1} \mathrm{H}$ NMR using an internal standard $(3:[4+5])$. Enantiomeric excess determined by GC or HPLC equipped with chiral columns. ${ }^{a} 48$ h. ${ }^{b} 72$ h. ${ }^{c} 120 \mathrm{~h}$. 


\section{(R)-3-([1,1'-Biphenyl]-4-yl)-N-benzylbut-3-en-2-amine 3aa}

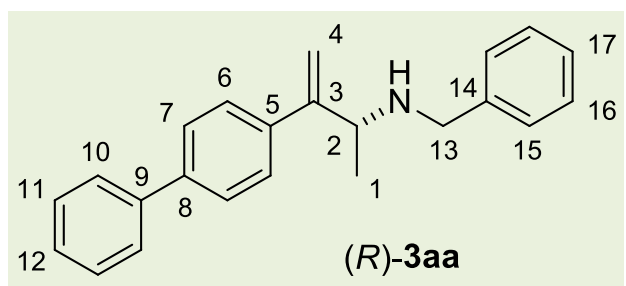

Synthesized at room temperature for $48 \mathrm{~h}$ following the general procedure using 4-(buta-1,3-dien-2-yl)1,1'-biphenyl 1a (51.6 mg, $0.25 \mathrm{mmol}, 1.0$ equiv), benzylamine 2a (109 $\mu \mathrm{L}, 1.00 \mathrm{mmol}, 4.0$ equiv), $\mathrm{Ni}(\operatorname{cod})_{2}$ (3.4 mg, $\left.0.0125 \mathrm{mmol}, 5 \mathrm{~mol} \%\right),(R, R)-$ BenzP* $^{*}(4.2 \mathrm{mg}, 0.015 \mathrm{mmol}, 6 \mathrm{~mol} \%)$, 2,2,2-trifluoroethanol (19 $\mu \mathrm{L}, 0.25 \mathrm{mmol}, 1.0$ equiv) and mesitylene $(0.50 \mathrm{~mL}, 0.5 \mathrm{M})$. Consumption of $1 \mathrm{a}:>99 \%$, conversion of 3aa: $79 \%$, r.r. $=$ 7:1. Purification by flash chromatography over silica gel (pentane:EtOAc $=5: 1$ ) led to the desired product as a yellow solid $(61.1 \mathrm{mg}, 78 \%$ yield, $93 \%$ ee) .

${ }^{1} \mathbf{H}$ NMR $\left(400 \mathrm{MHz}, \mathrm{CDCl}_{3}\right) \delta(\mathrm{ppm})=7.63\left(\mathrm{~d},{ }^{3} \mathrm{~J}_{\mathrm{HH}}=7.4 \mathrm{~Hz}, 2 \mathrm{H}, \mathrm{H}-10\right), 7.58\left(\mathrm{~d},{ }^{3} J_{\mathrm{HH}}=8.2\right.$ $\mathrm{Hz}, 2 \mathrm{H}, H-7), 7.52-7.43(\mathrm{~m}, 4 \mathrm{H}, \mathrm{H}-6+H-11), 7.40-7.25(\mathrm{~m}, 6 \mathrm{H}, \mathrm{H}-12+\mathrm{H}-15+\mathrm{H}-16+\mathrm{H}-17)$, $5.40(\mathrm{~s}, 1 \mathrm{H}, H-4), 5.38(\mathrm{~s}, 1 \mathrm{H}, H-4), 3.91\left(\mathrm{~d},{ }^{2} \mathrm{~J}_{\mathrm{HH}}=13.1 \mathrm{~Hz}, 1 \mathrm{H}, H-13\right), 3.86-3.73(\mathrm{~m}, 2 \mathrm{H}$, $\left.H-13^{\prime}+H-2\right), 1.65(\mathrm{~s}, 1 \mathrm{H}, \mathrm{NH}), 1.29\left(\mathrm{~d},{ }^{3} \mathrm{~J}_{\mathrm{HH}}=6.6 \mathrm{~Hz}, 3 \mathrm{H}, H-1\right)$.

${ }^{13} \mathrm{C}\left\{{ }^{1} \mathrm{H}\right\}$ NMR $\left(100 \mathrm{MHz}, \mathrm{CDCl}_{3}\right) \delta(\mathrm{ppm})=151.5(C-3), 140.9(C-9), 140.7(C-14), 140.3(C-$ 8), $140.2(C-5), 128.9(\mathrm{CH}-11), 128.5(\mathrm{CH}-16), 128.4(\mathrm{CH}-15), 127.5(\mathrm{CH}-6), 127.4(\mathrm{C}-12)$, $127.14(\mathrm{CH}-10), 127.06(\mathrm{CH}-7+\mathrm{CH}-17), 113.0\left(\mathrm{CH}_{2}-4\right), 56.7(\mathrm{CH}-2), 51.5\left(\mathrm{CH}_{2}-13\right), 21.8$ $\left(\mathrm{CH}_{3}-1\right)$.

m.p. $=44.2-46.0^{\circ} \mathrm{C}$.

HRMS (ESI+): calculated [M+H] $]^{+}$for $\mathrm{C}_{23} \mathrm{H}_{24} \mathrm{~N}^{+}:$314.1904; found: 314.1939.

IR (neat) $v\left(\mathrm{~cm}^{-1}\right): 3025,2924,2853,1623,1487,1452,1369,1152,1120,1077,1004,916$, $841,771,740,694$.

HPLC: $93 \%$ ee, chiral stationary column: OD-H, mobile phase: hexane/ $\mathrm{PrOH}=97 / 3,1.0$ $\mathrm{mL} / \mathrm{min}, 254 \mathrm{~nm}, 30^{\circ} \mathrm{C}, \mathrm{t}_{\mathrm{R}}$ (major) $=7.2 \mathrm{~min}, \mathrm{t}_{\mathrm{R}}($ minor $)=15.7 \mathrm{~min}$.

$[\alpha]^{20} \mathrm{D}=+7.0\left(c 1.0, \mathrm{CHCl}_{3}\right)$. 
<Chromatogram>

mAU

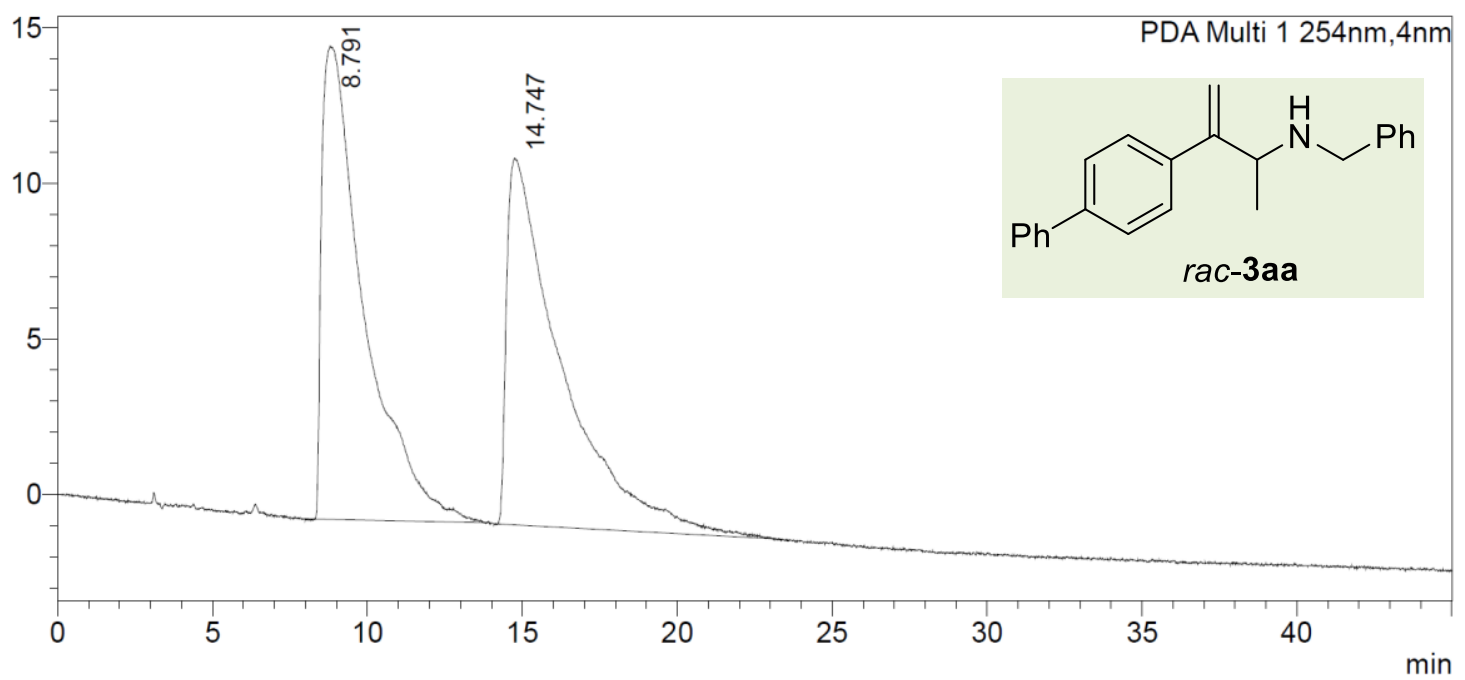

<Peak Table>

PDA Ch1 254nm

\begin{tabular}{|l|l}
\hline Peak\# Ret. Time & Area\% \\
\hline
\end{tabular}

\begin{tabular}{|r|r|r|}
\hline 1 & 8.791 & 49.954 \\
\hline 2 & 14.747 & 50.046 \\
\hline Total & & 100.000 \\
\hline
\end{tabular}

<Chromatogram>

mAU

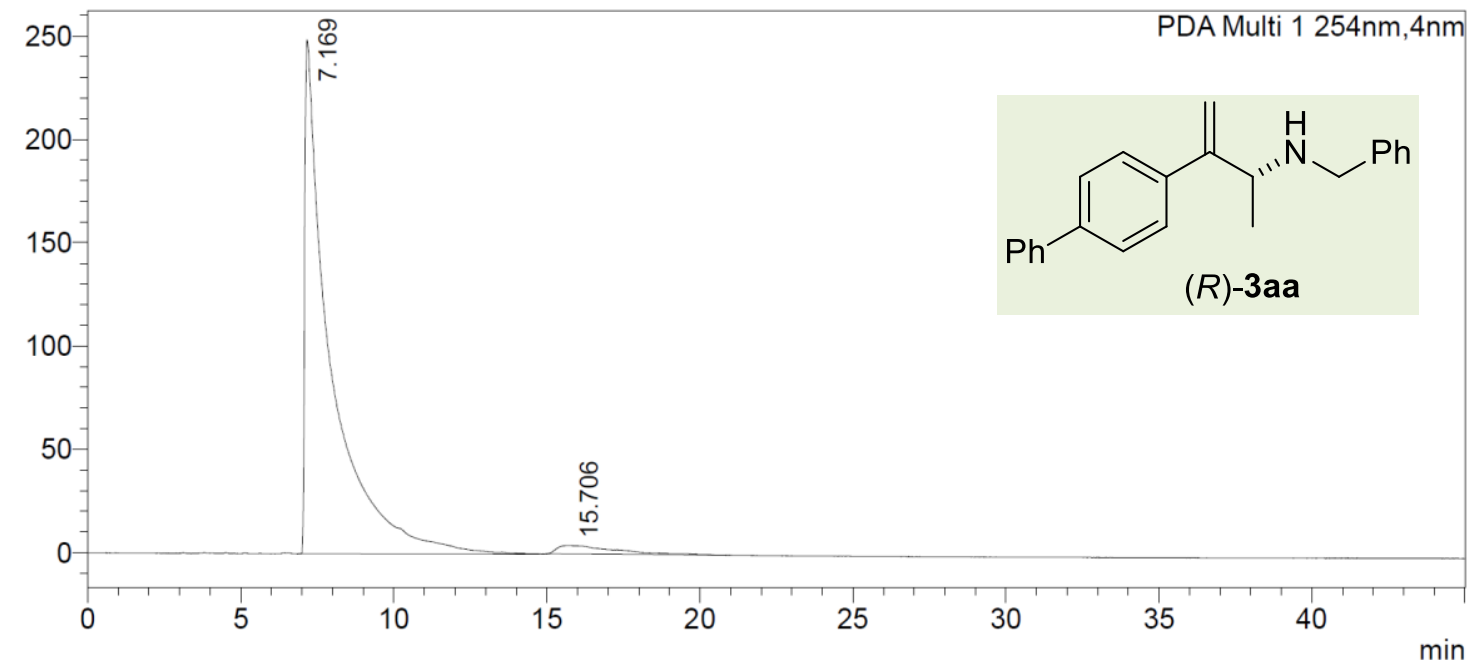

<Peak Table>

PDA Ch1 254nm

\begin{tabular}{|l|l} 
Peak\# Ret. Time & Area\%
\end{tabular}

\begin{tabular}{|r|r|r|}
\hline 1 & 7.169 & 96.402 \\
\hline 2 & 15.706 & 3.598 \\
\hline Total & & 100.000 \\
\hline
\end{tabular}




\section{(R)-N-Benzyl-3-phenylbut-3-en-2-amine 3ba}

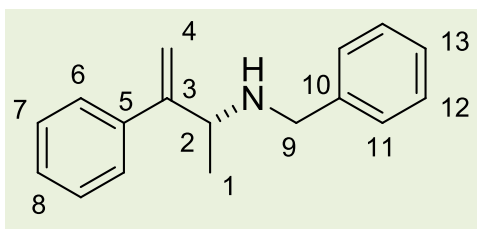

$(R)-3 \mathbf{b a}$

Synthesized at room temperature for $72 \mathrm{~h}$ following the general

procedure using buta-1,3-dien-2-ylbenzene $\mathbf{1 b}$ (33.0 $\mathrm{mg}, 0.25$ mmol, 1.0 equiv), benzylamine $2 \mathrm{a}(109 \mu \mathrm{L}, 1.00 \mathrm{mmol}, 4.0$ equiv), $\mathrm{Ni}(\mathrm{cod})_{2}$ (3.4 mg, $\left.0.0125 \mathrm{mmol}, 5 \mathrm{~mol} \%\right),(R, R)$-BenzP* (4.2 mg, $0.015 \mathrm{mmol}, 6 \mathrm{~mol} \%)$, 2,2,2-trifluoroethanol (19 $\mu \mathrm{L}$, $0.25 \mathrm{mmol}, 1.0$ equiv) and mesitylene $(0.50 \mathrm{~mL}, 0.5 \mathrm{M})$. Consumption of $\mathbf{1 b}$ : $>95 \%$, conversion of $3 \mathrm{ba}: 76 \%$, r.r. $=13: 1$. Purification by flash chromatography over silica gel (pentane:EtOAc $=4: 1)$ led to the desired product as a pale yellow oil $(45.0 \mathrm{mg}, 76 \%$ yield, $93 \%$ ee).

${ }^{1} \mathbf{H}$ NMR $\left(500 \mathrm{MHz}, \mathrm{CDCl}_{3}\right) \delta(\mathrm{ppm})=7.41-7.36(\mathrm{~m}, 2 \mathrm{H}, \mathrm{H}-A r), 7.35-7.22(\mathrm{~m}, 8 \mathrm{H}, \mathrm{H}-A r)$, $5.35-5.29(\mathrm{~m}, 2 \mathrm{H}, \mathrm{H}-4), 3.87\left(\mathrm{~d},{ }^{2} \mathrm{~J}_{\mathrm{HH}}=13.1 \mathrm{~Hz}, 1 \mathrm{H}, \mathrm{H}-9\right), 3.81-3.72(\mathrm{~m}, 2 \mathrm{H}, \mathrm{H}-9+\mathrm{H}-2)$, $1.51(\mathrm{~s}, 1 \mathrm{H}, \mathrm{NH}), 1.23\left(\mathrm{~d},{ }^{3} \mathrm{JH}=6.6 \mathrm{~Hz}, 3 \mathrm{H}, \mathrm{H}-1\right)$.

${ }^{13} \mathrm{C}\left\{{ }^{1} \mathrm{H}\right\}$ NMR $\left(130 \mathrm{MHz}, \mathrm{CDCl}_{3}\right) \delta(\mathrm{ppm})=152.0(C-3), 141.4(C-5), 140.8(C-10), 128.5(\mathrm{CH}-$ $\mathrm{Ar}), 128.3(2 \times \mathrm{CH}-\mathrm{Ar}), 127.5(\mathrm{CH}-8), 127.2(\mathrm{CH}-12), 127.0(\mathrm{CH}-13), 112.9\left(\mathrm{CH}_{2}-4\right), 56.8(\mathrm{CH}-$ 2), $51.5\left(\mathrm{CH}_{2}-9\right), 21.8\left(\mathrm{CH}_{3}-1\right)$.

HRMS (ESI ${ }^{+}$: calculated $[\mathrm{M}+\mathrm{H}]^{+}$for $\mathrm{C}_{17} \mathrm{H}_{20} \mathrm{~N}^{+}: 238.1591$; found: 238.1590 .

IR (neat) $v\left(\mathrm{~cm}^{-1}\right): 3027,2967,2929,1627,1600,1493,1452,1369,1315,1150,1072,1027$, 906, 778, 734, 695.

Chiral GC: $93 \%$ ee, chiral stationary column: Hydrodex $\gamma$-DiMOM, mobile phase: $\mathrm{H}_{2}$ (45 $\mathrm{cm} / \mathrm{s}$ ), temperature gradient: $145{ }^{\circ} \mathrm{C}$ isothermal, $t_{R}($ minor $)=153.5 \mathrm{~min}, t_{R}$ (major) $=155.1$ $\min$.

$[\alpha]^{20}=+3.1\left(c 0.47, \mathrm{CH}_{2} \mathrm{Cl}_{2}\right)$. 

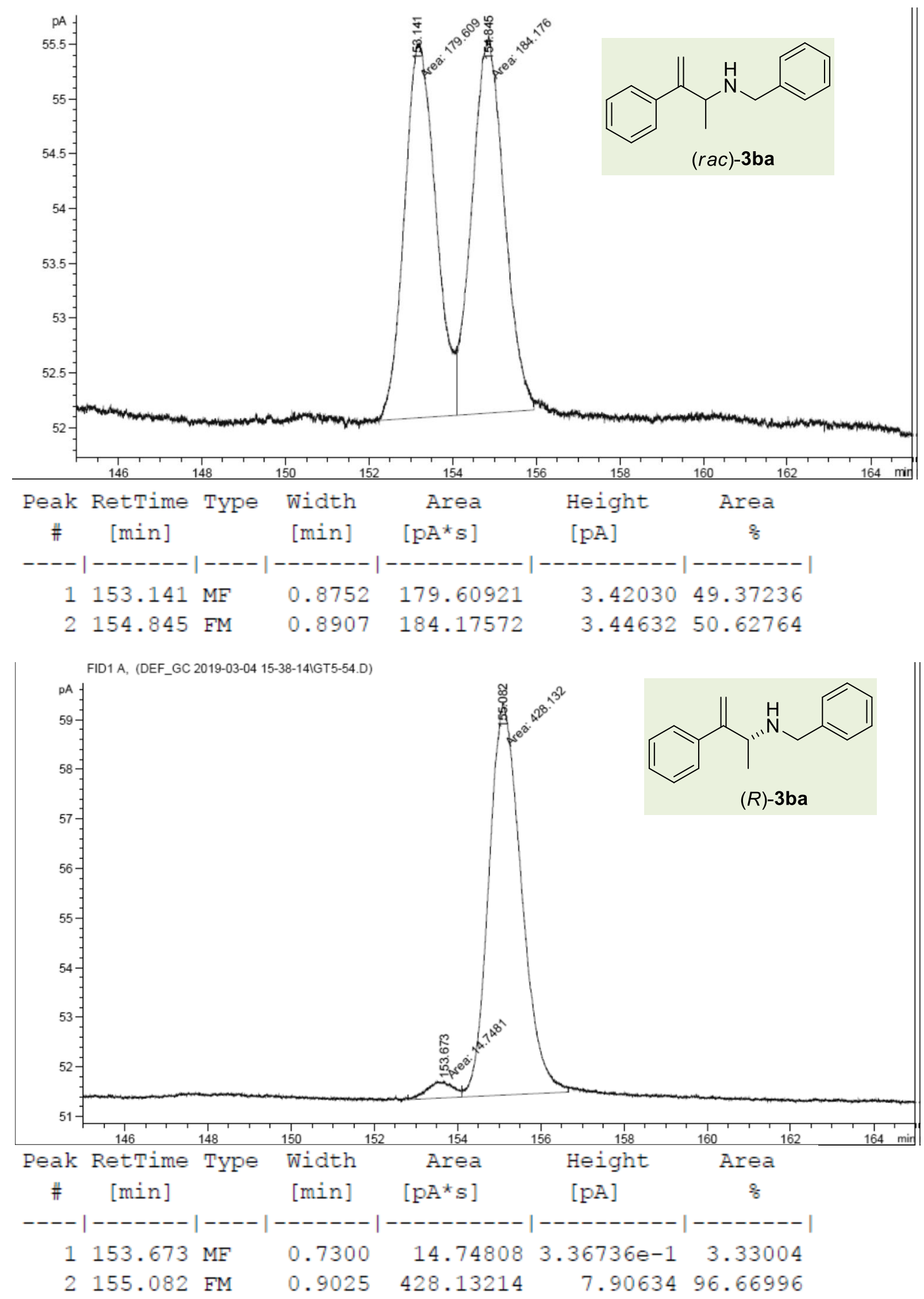


\section{(R)-N-Benzyl-3-(4-isobutylphenyl)but-3-en-2-amine 3ca}

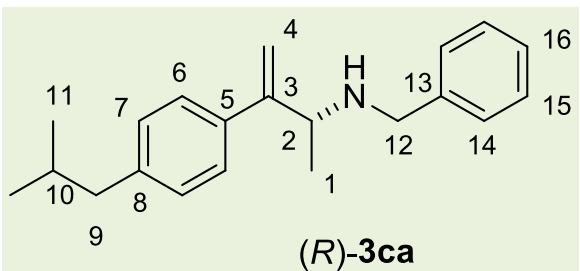

Synthesized at room temperature for $48 \mathrm{~h}$ following the general procedure using 1-(buta-1,3-dien-2-yl)-4isobutylbenzene 1c $(75.0 \mathrm{mg}, 0.40 \mathrm{mmol}, 1.0$ equiv), benzylamine 2a (175 $\mu \mathrm{L}, 1.60 \mathrm{mmol}, 4.0$ equiv), $\mathrm{Ni}(\mathrm{cod})_{2}$ $(5.5 \mathrm{mg}, 0.020 \mathrm{mmol}, 5 \mathrm{~mol} \%),(R, R)-$ BenzP* $^{*}(6.8 \mathrm{mg}$, $0.024 \mathrm{mmol}, 6 \mathrm{~mol} \%), 2,2,2$-trifluoroethanol (29 $\mu \mathrm{L}, 0.40 \mathrm{mmol}, 1.0$ equiv) and mesitylene $(0.80 \mathrm{~mL}, 0.5 \mathrm{M})$. Consumption of 1c: $>95 \%$, conversion of $3 \mathrm{ca}: 84 \%$, r.r. $=8: 1$. Purification by flash chromatography over silica gel (pentane:EtOAc $=4: 1$ ) led to the desired product as a pale yellow oil ( $99.0 \mathrm{mg}, 84 \%$ yield, $91 \%$ ee).

${ }^{1} \mathrm{H}$ NMR $\left(500 \mathrm{MHz}, \mathrm{CDCl}_{3}\right) \delta(\mathrm{ppm})=7.34-7.27(\mathrm{~m}, 6 \mathrm{H}, \mathrm{H}-15+\mathrm{H}-14+\mathrm{H}-6), 7.26-7.22$ $(\mathrm{m}, 1 \mathrm{H}, \mathrm{H}-16), 7.10\left(\mathrm{~d},{ }^{3} \mathrm{JHH}_{\mathrm{HH}}=8.1 \mathrm{~Hz}, 2 \mathrm{H}, \mathrm{H}-7\right), 5.33-5.28(\mathrm{~m}, 2 \mathrm{H}, \mathrm{H}-4), 3.85\left(\mathrm{~d},{ }^{2}{ }^{\mathrm{HH}}=\right.$ 13.1 Hz, 1H, H-12), $3.79-3.71(\mathrm{~m}, 2 \mathrm{H}, \mathrm{H}-2+\mathrm{H}-12), 2.47\left(\mathrm{~d},{ }^{3} \mathrm{~J}_{\mathrm{HH}}=7.2 \mathrm{~Hz}, 3 \mathrm{H}, \mathrm{H}-9\right), 1.87$ (heptapp, $\left.{ }^{3} \mathrm{JHH}_{\mathrm{HH}}=6.6 \mathrm{~Hz}, 1 \mathrm{H}, \mathrm{H}-10\right), 1.52(\mathrm{~s}, 1 \mathrm{H}, \mathrm{N}-\mathrm{H}), 1.24\left(\mathrm{~d},{ }^{3} \mathrm{~J}_{\mathrm{HH}}=6.6 \mathrm{~Hz}, 3 \mathrm{H}, \mathrm{H}-1\right), 0.92$ (d, ${ }^{3} \mathrm{JHH}=6.6 \mathrm{~Hz}, 6 \mathrm{H}, \mathrm{H}-11$ )

${ }^{13} \mathrm{C}\left\{{ }^{1} \mathrm{H}\right\}$ NMR $\left(130 \mathrm{MHz}, \mathrm{CDCl}_{3}\right) \delta(\mathrm{ppm})=151.9(C-3), 141.1(C-8), 140.9(C-13), 129.1(C-$ 5), $128.5(\mathrm{CH}-\mathrm{Ar}), 128.4(\mathrm{CH}-\mathrm{Ar}), 1267.0(\mathrm{CH}-16), 126.8(\mathrm{CH}-\mathrm{Ar}), 112.2\left(\mathrm{CH}_{2}-4\right), 56.7(\mathrm{CH}-$ 2), $51.5\left(\mathrm{CH}_{2}-12\right), 45.2\left(\mathrm{CH}_{2}-9\right), 30.4(\mathrm{CH}-10), 22.6\left(\mathrm{CH}_{3}-11\right), 21.8\left(\mathrm{CH}_{3}-1\right)$.

HRMS (ESI+): calculated $[\mathrm{M}+\mathrm{H}]^{+}$for $\mathrm{C}_{21} \mathrm{H}_{28} \mathrm{~N}^{+}:$:294.2217; found: 294.2247 .

IR (neat) $\vee\left(\mathrm{cm}^{-1}\right): 3026,2955,1625,1510,1495,1454,1367,1151,1120,906,847,801$, $731,697$.

HPLC: $91 \%$ ee, chiral stationary column: AD-H, mobile phase: hexane/ $\mathrm{PrOH}=99 / 1,1.0$ $\mathrm{mL} / \mathrm{min}, 254 \mathrm{~nm}, 30^{\circ} \mathrm{C}, \mathrm{t}_{\mathrm{R}}($ minor $)=5.3 \mathrm{~min}, \mathrm{t}_{\mathrm{R}}$ (major) $=5.9 \mathrm{~min}$.

$[\alpha]^{20} \mathrm{D}=+2.8\left(c 0.74, \mathrm{CHCl}_{3}\right)$. 
$<$ Chromatogram>

mAU

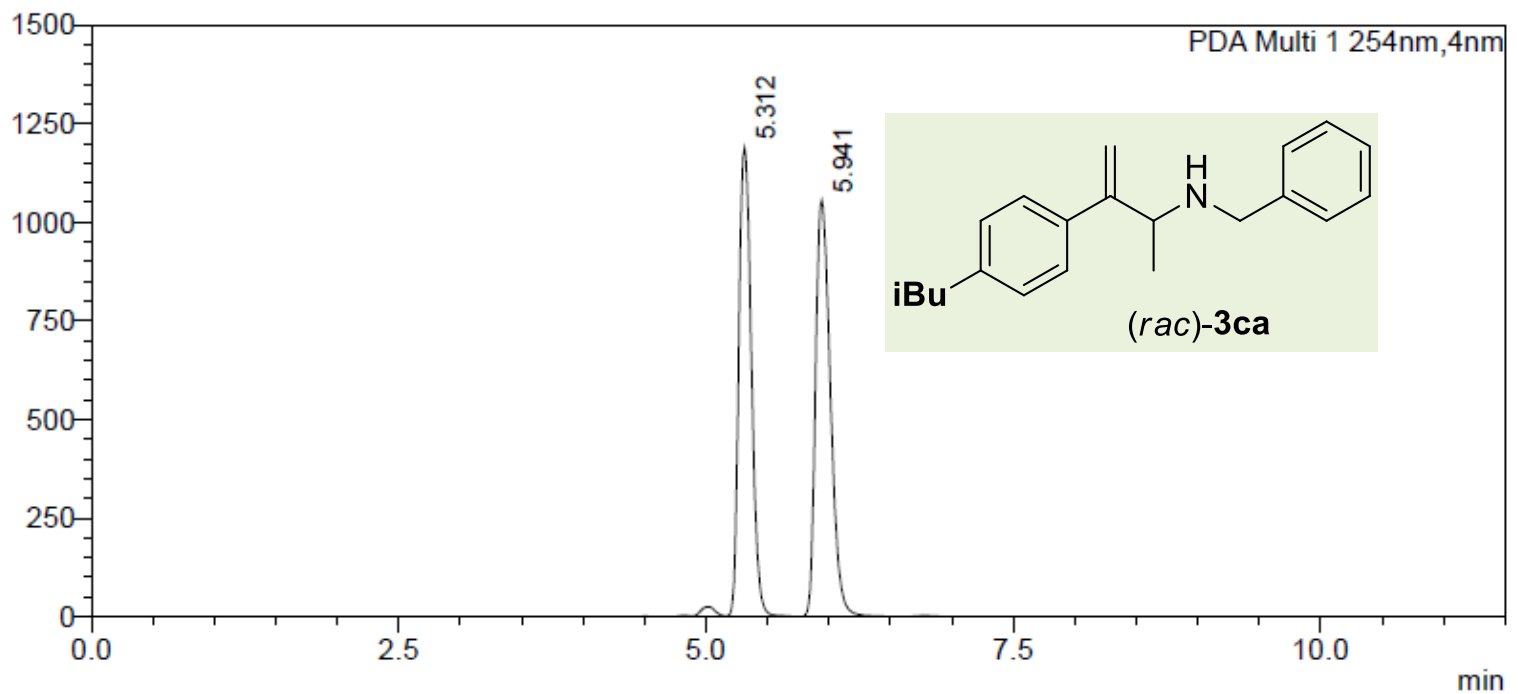

PDA Ch1 254nm

\begin{tabular}{|l|l|}
\hline Peak\# & Ret. Time \\
\hline
\end{tabular}

\begin{tabular}{|r|r|r|}
\hline 1 & 5.312 & 48.725 \\
\hline 2 & 5.941 & 51.275 \\
\hline Total & & 100.000 \\
\hline
\end{tabular}

$<$ Chromatogram $>$

mAU

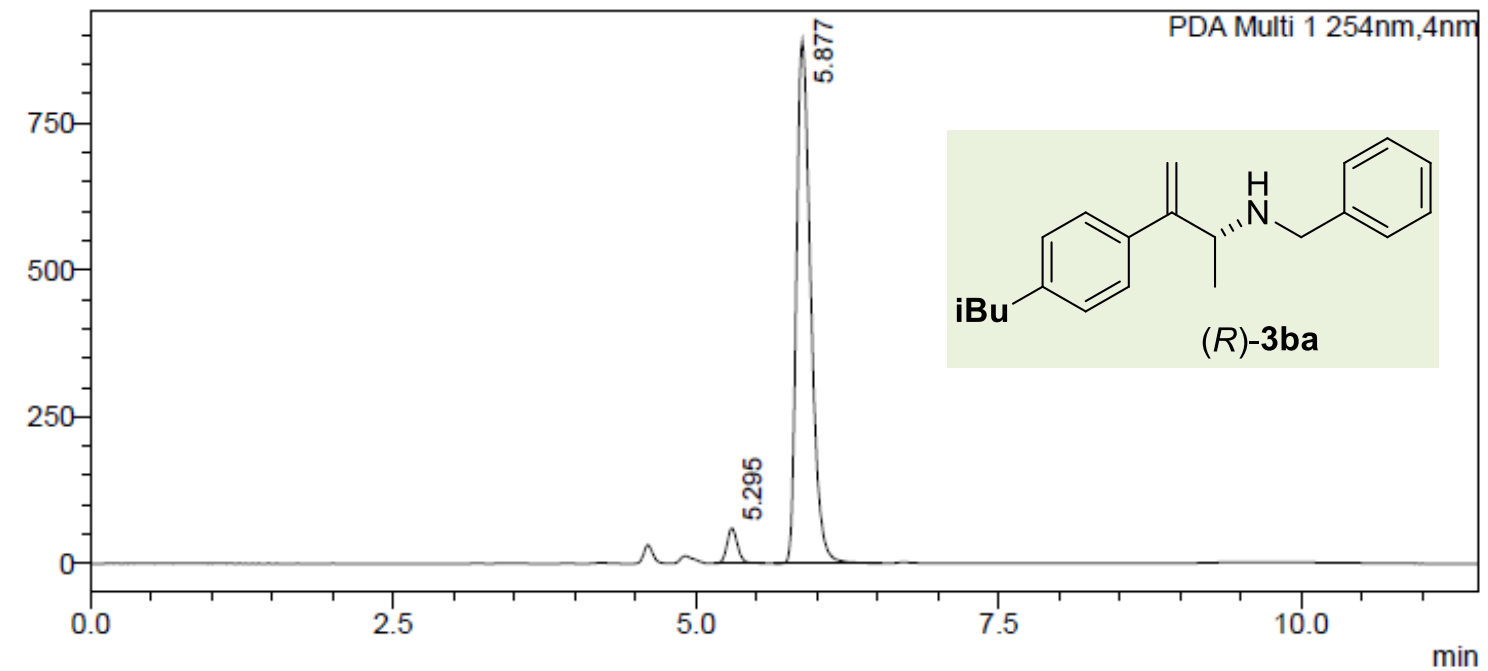

<Peak Table>

PDA Ch1 254nm

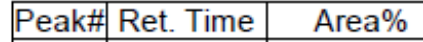

\begin{tabular}{|r|r|r|}
\hline 1 & 5.295 & 4.721 \\
\hline 2 & 5.877 & 95.279 \\
\hline Total & & 100.000 \\
\hline
\end{tabular}


(R)-N-Benzyl-3-(4-methoxyphenyl)but-3-en-2-amine 3da

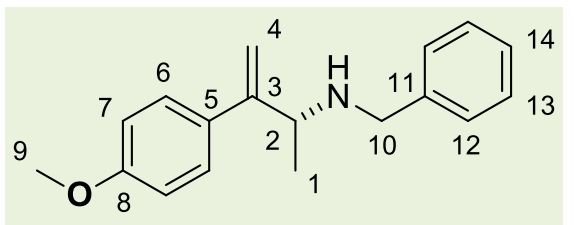

$(R)-3 \mathrm{da}$

Synthesized at room temperature for $120 \mathrm{~h}$ following the general procedure using 1-(buta-1,3-dien-2-yl)-4methoxybenzene 1d (40.0 mg, $0.25 \mathrm{mmol}, 1.0$ equiv), benzylamine 2a $\left(110 \mu \mathrm{L}, 1.00 \mathrm{mmol}, 4.0\right.$ equiv), $\mathrm{Ni}(\operatorname{cod})_{2}$ (3.4 mg, $0.0125 \mathrm{mmol}, 5 \mathrm{~mol} \%),(R, R)$-BenzP* (4.2 mg, $0.015 \mathrm{mmol}, 6 \mathrm{~mol} \%), 2,2,2$-trifluoroethanol (19 $\mu \mathrm{L}, 0.25 \mathrm{mmol}, 1.0$ equiv) and mesitylene $(0.50 \mathrm{~mL}, 0.5 \mathrm{M})$. Consumption of $\mathbf{1 d :} 91 \%$, conversion of $3 \mathrm{da}: 81 \%, r . r .=12: 1$. Purification by flash chromatography over silica gel (pentane:EtOAc $=3: 2$ ) led to the desired product as a pale yellow oil $(52.0 \mathrm{mg}, 79 \%$ yield, $90 \%$ ee).

${ }^{1} \mathbf{H}$ NMR $\left(500 \mathrm{MHz}, \mathrm{CDCl}_{3}\right) \delta(\mathrm{ppm})=7.37-7.29(\mathrm{~m}, 6 \mathrm{H}, \mathrm{H}-6+\mathrm{H}-12+\mathrm{H}-13), 7.26-7.22$ $(\mathrm{m}, 1 \mathrm{H}, \mathrm{H}-14), 6.88-6.83(\mathrm{~m}, 2 \mathrm{H}, \mathrm{H}-7), 5.26-5.24(\mathrm{~m}, 2 \mathrm{H}, \mathrm{H}-4), 3.85\left(\mathrm{~d},{ }^{2} \mathrm{~J}_{\mathrm{HH}}=13.1 \mathrm{~Hz}\right.$, $1 \mathrm{H}, \mathrm{H}-10), 3.82(\mathrm{~s}, 3 \mathrm{H}, \mathrm{H}-9), 3.76\left(\mathrm{~d},{ }^{2} \mathrm{JHH}_{\mathrm{HH}}=13.1 \mathrm{~Hz}, 1 \mathrm{H}, \mathrm{H}-10\right), 3.72\left(\mathrm{q},{ }^{3} \mathrm{JHH}_{\mathrm{HH}}=6.6 \mathrm{~Hz}, 1 \mathrm{H}\right.$, $\mathrm{H}-2), 1.53(\mathrm{~s}, 1 \mathrm{H}, \mathrm{N}-H), 1.23\left(\mathrm{~d},{ }^{3} \mathrm{~J}_{\mathrm{HH}}=6.6 \mathrm{~Hz}, 3 \mathrm{H}, \mathrm{H}-1\right)$.

${ }^{13} \mathrm{C}\left\{{ }^{1} \mathrm{H}\right\}$ NMR $\left(130 \mathrm{MHz}, \mathrm{CDCl}_{3}\right) \delta(\mathrm{ppm})=159.2(C-8), 151.3(C-3), 140.9(C-11), 133.7$ (C-5), $128.5(\mathrm{CH}-A r), 128.3(\mathrm{CH}-A r), 128.2(\mathrm{CH}-A r), 127.0(\mathrm{CH}-14), 113.7\left(\mathrm{CH}_{2}-7\right), 111.7$ $(\mathrm{CH}-4), 56.8(\mathrm{CH}-2), 55.4\left(\mathrm{CH}_{3}-9\right), 51.5\left(\mathrm{CH}_{2}-10\right), 21.7\left(\mathrm{CH}_{3}-1\right)$.

HRMS $\left(\mathrm{ESI}^{+}\right)$: calculated $[\mathrm{M}+\mathrm{H}]^{+}$for $\mathrm{C}_{18} \mathrm{H}_{22} \mathrm{NO}^{+}:$268.1696; found: 268.1731.

IR (neat) $v\left(\mathrm{~cm}^{-1}\right):$ 3029, 2928, 1607, 1509, 1454, 1369, 1292, 1243, 1179, 1150, 1113, 1032, 903, 834, 733, 697.

HPLC: $90 \%$ ee, chiral stationary column: $A D-H$, mobile phase: hexane/ $\mathrm{PrOH}=98 / 2,1.0$ $\mathrm{mL} / \mathrm{min}, 290 \mathrm{~nm}, 30^{\circ} \mathrm{C}, \mathrm{t}_{\mathrm{R}}($ minor $)=6.0 \mathrm{~min}, \mathrm{t}_{\mathrm{R}}$ (major) $=6.6 \mathrm{~min}$.

$[\alpha]^{20} \mathrm{D}=+4.2\left(c 1.0, \mathrm{CH}_{2} \mathrm{Cl}_{2}\right)$. 
$<$ Cnromatogram>

mAU

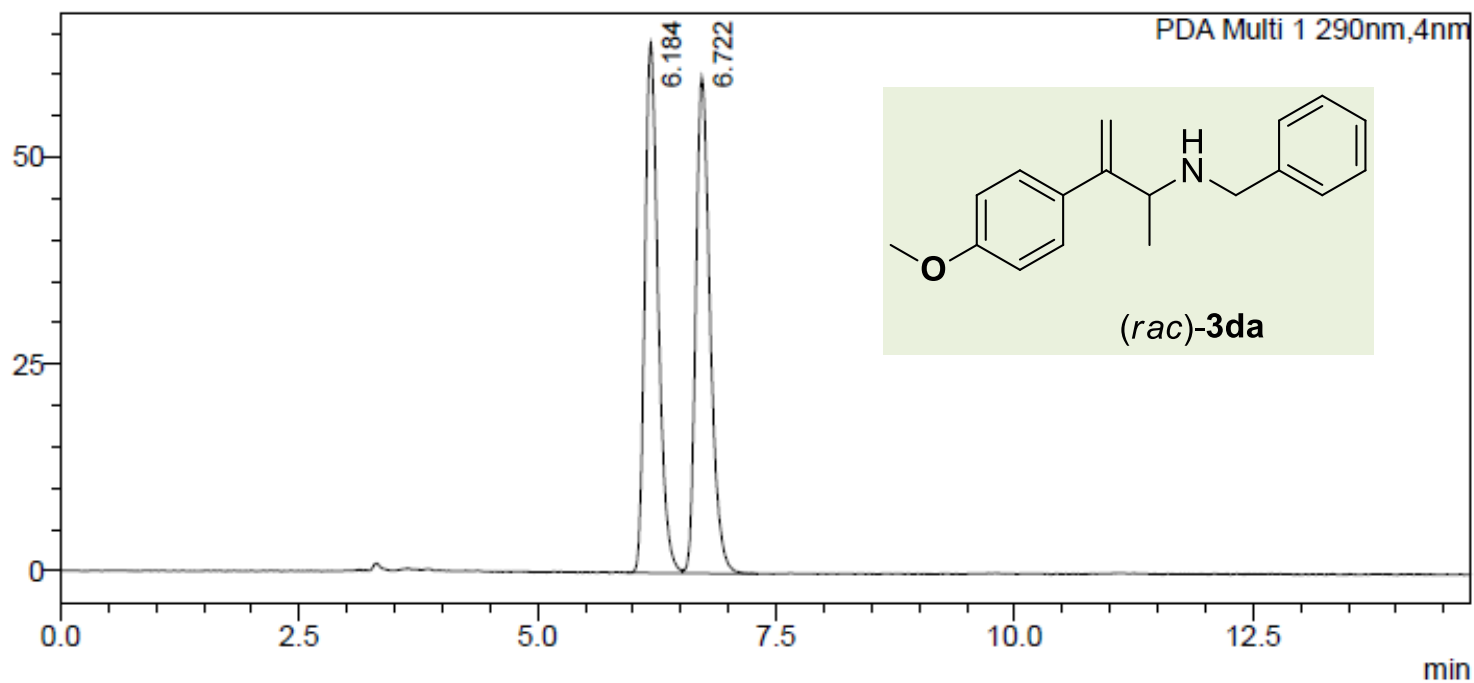

<Peak Table>

PDA Ch1 290nm

\begin{tabular}{|r|r|}
\hline Peak\# & Ret. Time \\
\hline
\end{tabular}

\begin{tabular}{|c|c|c|}
\hline 1 & 6104 & 40908 \\
\hline 2 & $\frac{0.104}{6727}$ & \\
\hline$\frac{2}{\text { Total }}$ & 0.122 & 100.000 \\
\hline
\end{tabular}

$<$ Chromatogram>

mAU

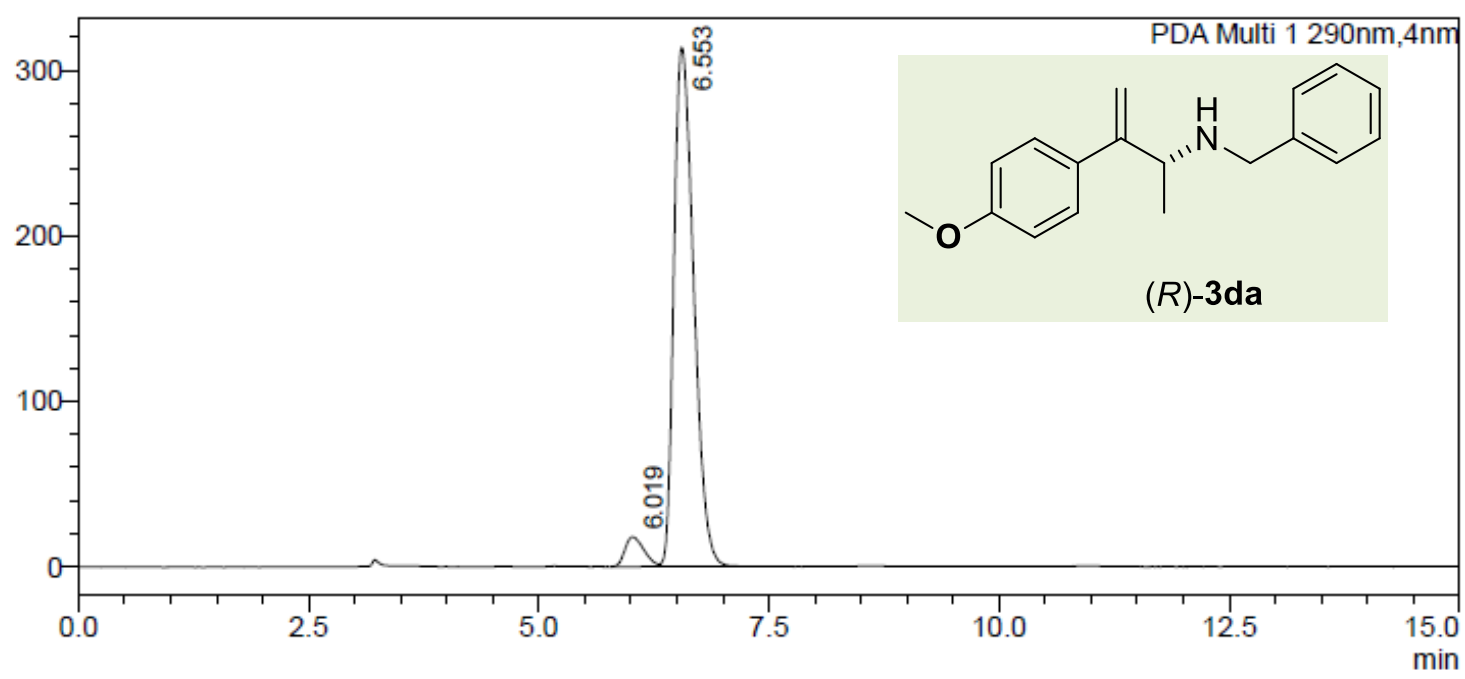

<Peak Table>

PDA Ch1 290nm

\begin{tabular}{|r|r|r|}
\hline Peak\# & Ret. Time & \multicolumn{1}{c|}{ Area\% } \\
\hline 1 & 6.019 & 5.266 \\
\hline 2 & 6.553 & 94.734 \\
\hline Total & & 100.000 \\
\hline
\end{tabular}




\section{(R)-N-Benzyl-3-(4-(trifluoromethyl)phenyl)but-3-en-2-amine 3ea}

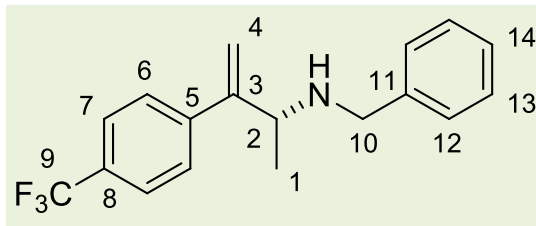

$(R)-3$ ea

Synthesized at room temperature for $72 \mathrm{~h}$ following the general procedure using 1-(buta-1,3-dien-2-yl)-4(trifluoromethyl)benzene 1 e $(50.0 \mathrm{mg}, 0.25 \mathrm{mmol}, 1.0$ equiv), benzylamine $2 \mathrm{a}(110 \mu \mathrm{L}, 1.00 \mathrm{mmol}, 4.0$ equiv), $\mathrm{Ni}(\operatorname{cod})_{2}$ (3.4 mg, $\left.0.0125 \mathrm{mmol}, 5 \mathrm{~mol} \%\right),(R, R)-B e n z \mathrm{P}^{*}$ (4.2 mg, $0.015 \mathrm{mmol}, 6 \mathrm{~mol} \%$ ), 2,2,2-trifluoroethanol (19 $\mu \mathrm{L}, 0.25 \mathrm{mmol}, 1.0$ equiv) and mesitylene $(0.50 \mathrm{~mL}, 0.5 \mathrm{M})$. Consumption of 1e: $>95 \%$, conversion of 3ea: $83 \%$, r.r. $=6: 1$. Purification by flash chromatography over silica gel (pentane:EtOAc $=4: 1$ ) led to the desired product as a pale yellow oil ( $59.0 \mathrm{mg}, 77 \%$ yield, $95 \%$ ee).

${ }^{1} \mathrm{H}$ NMR $\left(500 \mathrm{MHz}, \mathrm{CDCl}_{3}\right) \delta(\mathrm{ppm})=7.57\left(\mathrm{~d},{ }^{3} J_{\mathrm{HH}}=8.2 \mathrm{~Hz}, 2 \mathrm{H}, \mathrm{H}-7\right), 7.51\left(\mathrm{~d},{ }^{3} J_{\mathrm{HH}}=8.2 \mathrm{~Hz}\right.$, $2 \mathrm{H}, \mathrm{H}-6), 7.35-7.28(\mathrm{~m}, 4 \mathrm{H}, \mathrm{H}-12+\mathrm{H}-13), 7.27-7.22(\mathrm{~m}, 1 \mathrm{H}, \mathrm{H}-14), 5.41\left(\mathrm{t},{ }^{2} J_{\mathrm{HH}}=1.1 \mathrm{~Hz}\right.$, $2 \mathrm{H}, \mathrm{H}-4), 5.35\left(\mathrm{~d},{ }^{2} J_{\mathrm{HH}}=1.1 \mathrm{~Hz}, 1 \mathrm{H}, \mathrm{H}-4\right), 3.87\left(\mathrm{~d},{ }^{2} J_{\mathrm{HH}}=13.2 \mathrm{~Hz}, 1 \mathrm{H}, \mathrm{H}-10\right), 3.81-3.70(\mathrm{~m}$, $2 \mathrm{H}, \mathrm{H}-10+\mathrm{H}-2), 1.44(\mathrm{~s}, 1 \mathrm{H}, \mathrm{N}-\mathrm{H}), 1.22\left(\mathrm{~d},{ }^{3} \mathrm{~J}_{\mathrm{HH}}=6.6 \mathrm{~Hz}, 3 \mathrm{H}, \mathrm{H}-1\right)$.

${ }^{13} \mathbf{C}\left\{{ }^{1} \mathrm{H}\right\}$ NMR $\left(130 \mathrm{MHz}, \mathrm{CDCl}_{3}\right) \delta(\mathrm{ppm})=151.3(C-3), 145.1(C-5), 140.8(C-11), 129.7$ $\left({ }^{2} J_{\mathrm{CF}}, \mathrm{q}, J=32 \mathrm{~Hz}, \mathrm{C}-8\right), 128.6(\mathrm{CH}-\mathrm{Ar}), 128.3(\mathrm{CH}-\mathrm{Ar}), 127.7(\mathrm{CH}-6), 127.1(\mathrm{CH}-14), 125.2$ $\left({ }^{3} J_{\mathrm{CF}}, J=4 \mathrm{~Hz}, \mathrm{CH}-7\right), 124.5\left(\mathrm{q},{ }^{1} \mathrm{~J}_{\mathrm{CF}}=272 \mathrm{~Hz}, C-9\right), 57.2(\mathrm{CH}-2), 51.6\left(\mathrm{CH}_{2}-10\right), 21.7$ $\left(\mathrm{CH}_{3}-1\right)$.

${ }^{19} \mathrm{~F}\left\{{ }^{1} \mathrm{H}\right\}$ NMR $\left(280 \mathrm{MHz}, \mathrm{CDCl}_{3}\right) \delta(\mathrm{ppm})=-62.49$.

HRMS $\left(\mathrm{ESI}^{+}\right)$: calculated $[\mathrm{M}+\mathrm{H}]^{+}$for $\mathrm{C}_{18} \mathrm{H}_{19} \mathrm{~F}_{3} \mathrm{~N}^{+}:$:306.1465; found: 306.1461 .

IR (neat) $v\left(\mathrm{~cm}^{-1}\right): 3030,2970,1616,1495,1453,1404,1372,1322,1163,1118,1065,1015$, $915,846,735,698$.

Chiral GC: $95 \%$ ee, chiral stationary column: Hydrodex $\beta-3 P$, mobile phase: $\mathrm{H}_{2}(45 \mathrm{~cm} / \mathrm{s})$, temperature gradient: $130{ }^{\circ} \mathrm{C}$ isothermal, $t_{R}($ minor $)=90.9 \mathrm{~min}, t_{R}($ major $)=93.1 \mathrm{~min}$.

$[\alpha]^{20}=+6.6\left(c 1.34, \mathrm{CH}_{2} \mathrm{Cl}_{2}\right)$. 


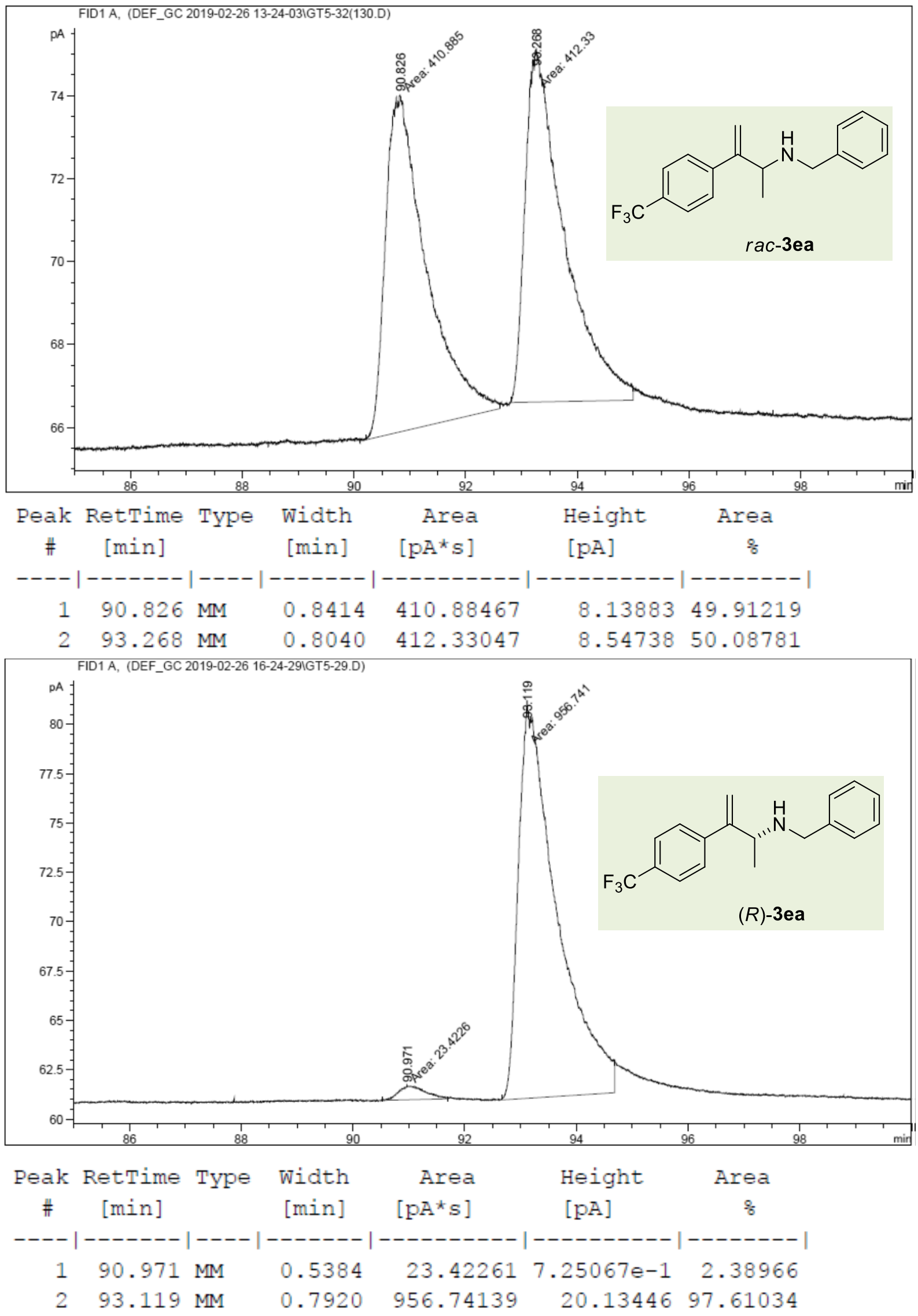




\section{(R)-N-Benzyl-3-(3-methoxyphenyl)but-3-en-2-amine 3fa}

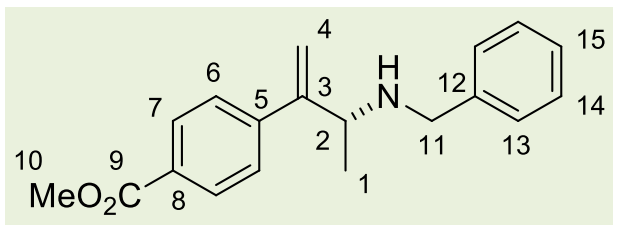

$(R)-3$ fa

Synthesized at room temperature for $72 \mathrm{~h}$ following the general procedure using methyl 4-(buta-1,3-dien-2yl)benzoate 1 f $(47.0 \mathrm{mg}, 0.25 \mathrm{mmol}, 1.0$ equiv), benzylamine 2a (110 $\mathrm{LL}, 1.00 \mathrm{mmol}, 4.0$ equiv), $\mathrm{Ni}(\operatorname{cod})_{2}(3.4 \mathrm{mg}, 0.0125 \mathrm{mmol}, 5 \mathrm{~mol} \%),(R, R)$-BenzP* (4.2 mg, $0.015 \mathrm{mmol}, 6 \mathrm{~mol} \%$ ), 2,2,2-trifluoroethanol (19 $\mu \mathrm{L}, 0.25 \mathrm{mmol}, 1.0$ equiv) and mesitylene $(0.50 \mathrm{~mL}, 0.5 \mathrm{M})$. Consumption of 1f: $>95 \%$, conversion of $3 \mathbf{f a :} 85 \%$, r.r. $=5.4: 1$. Purification by flash chromatography over silica gel (pentane:EtOAc $=4: 1$ ) led to the desired product as a pale yellow oil ( $60.0 \mathrm{mg}, 81 \%$ yield, $92 \%$ ee).

${ }^{1} \mathrm{H}$ NMR $\left(500 \mathrm{MHz}, \mathrm{CDCl}_{3}\right) \delta(\mathrm{ppm})=8.01-7.95(\mathrm{~m}, 2 \mathrm{H}, \mathrm{H}-7), 7.46\left(\mathrm{~d},{ }^{3} \mathrm{~J}_{\mathrm{HH}}=8.4 \mathrm{~Hz}, 2 \mathrm{H}, \mathrm{H}-\right.$ 6), $7.36-7.29(\mathrm{~m}, 4 \mathrm{H}, \mathrm{H}-13+\mathrm{H}-14), 7.28-7.22(\mathrm{~m}, 1 \mathrm{H}, \mathrm{H}-15), 5.42(\mathrm{~s}, 1 \mathrm{H}, \mathrm{H}-4), 5.39(\mathrm{~s}$, $1 \mathrm{H}, \mathrm{H}-4), 3.92(\mathrm{~s}, 3 \mathrm{H}, \mathrm{H}-10), 3.87\left(\mathrm{~d},{ }^{2}{ }^{\mathrm{HH}}=13.1 \mathrm{~Hz}, 1 \mathrm{H}, \mathrm{H}-11\right), 3.79-3.69(\mathrm{~m}, 2 \mathrm{H}, \mathrm{H}-2+\mathrm{H}-$ 11), $1.51(\mathrm{~s}, 1 \mathrm{H}, \mathrm{N}-\mathrm{H}), 1.22\left(\mathrm{~d},{ }^{3} \mathrm{~J}_{\mathrm{HH}}=6.6 \mathrm{~Hz}, 3 \mathrm{H}, \mathrm{H}-1\right)$.

${ }^{13} \mathbf{C}\left\{{ }^{1} \mathrm{H}\right\}$ NMR $\left(130 \mathrm{MHz}, \mathrm{CDCl}_{3}\right) \delta(\mathrm{ppm})=167.1(C-9), 151.2(C-3), 146.0(C-5), 140.6$ (C-12), $129.7(\mathrm{CH}-7), 129.2(\mathrm{C}-8), 128.6(\mathrm{CH}-\mathrm{Ar}), 128.3(\mathrm{CH}-\mathrm{Ar}), 127.17(\mathrm{CH}-\mathrm{Ar}), 127.15$ $(\mathrm{CH}-\mathrm{Ar}), 114.6\left(\mathrm{CH}_{2}-4\right), 56.7(\mathrm{CH}-2), 52.2\left(\mathrm{CH}_{2}-11\right), 51.4\left(\mathrm{CH}_{3}-10\right), 21.7\left(\mathrm{CH}_{3}-1\right)$.

HRMS $\left(E S I^{+}\right)$: calculated $[\mathrm{M}+\mathrm{H}]^{+}$for $\mathrm{C}_{19} \mathrm{H}_{21} \mathrm{NO}_{2}{ }^{+}$: 296.1645; found: 296.1646.

IR (neat) $\vee\left(\mathrm{cm}^{-1}\right): 3028,2953,1718,1601,1435,1274,1183,1107,1017,967,912,862$, $782,697$.

HPLC: $92 \%$ ee, chiral stationary column: OD-H, mobile phase: hexane/ $\mathrm{PrOH}=95 / 5,1.0$ $\mathrm{mL} / \mathrm{min}, 290 \mathrm{~nm}, 30^{\circ} \mathrm{C}, \mathrm{t}_{\mathrm{R}}$ (major) $=7.8 \mathrm{~min}, \mathrm{t}_{\mathrm{R}}($ minor $)=9.9 \mathrm{~min}$.

$[\alpha]^{20}=+3.5\left(c 0.96, \mathrm{CH}_{2} \mathrm{Cl}_{2}\right)$. 
$<$ Chromatogram $>$

mAU

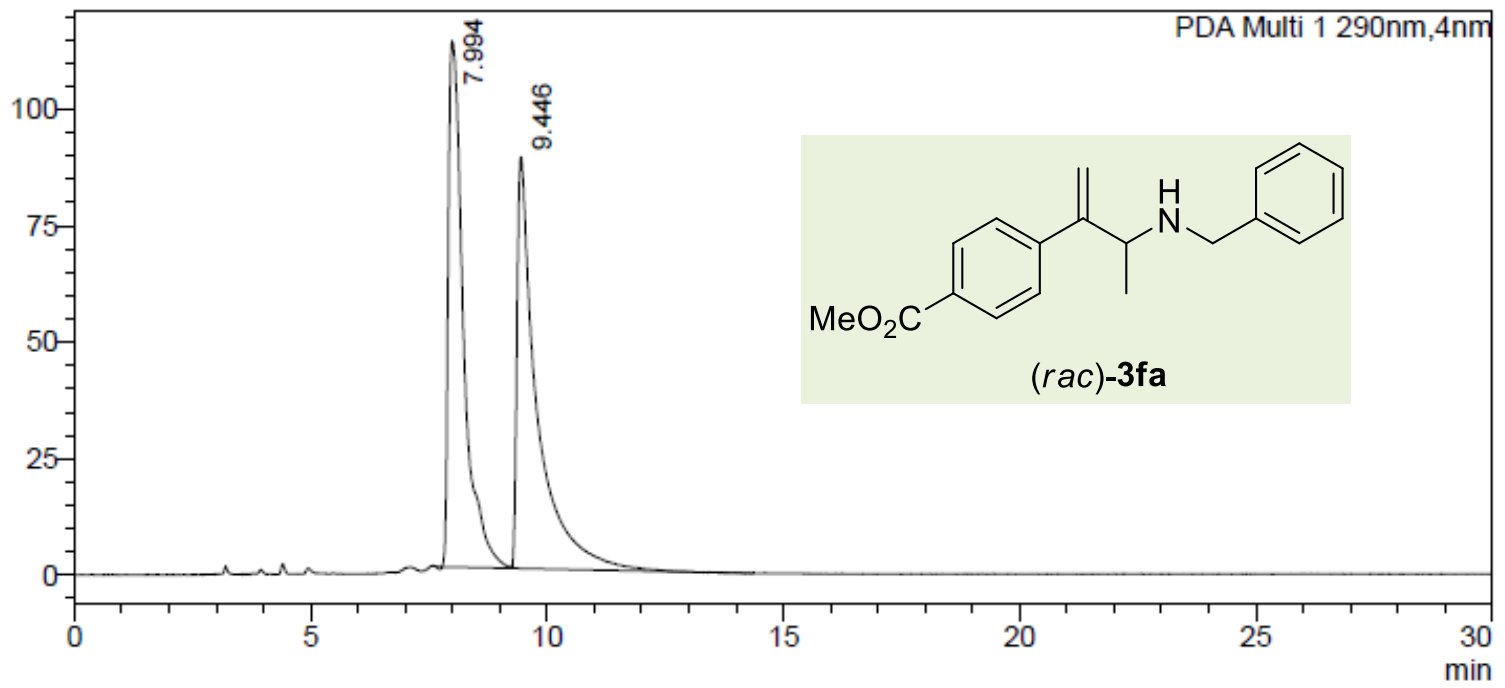

<Peak Table>

PDA Ch1 290nm

Peak\# Ret. Time $\quad$ Area\%

\begin{tabular}{|r|r|r|}
\hline 1 & 7.994 & 49.271 \\
\hline 2 & 9.446 & 50.729 \\
\hline Total & & 100.000 \\
\hline
\end{tabular}

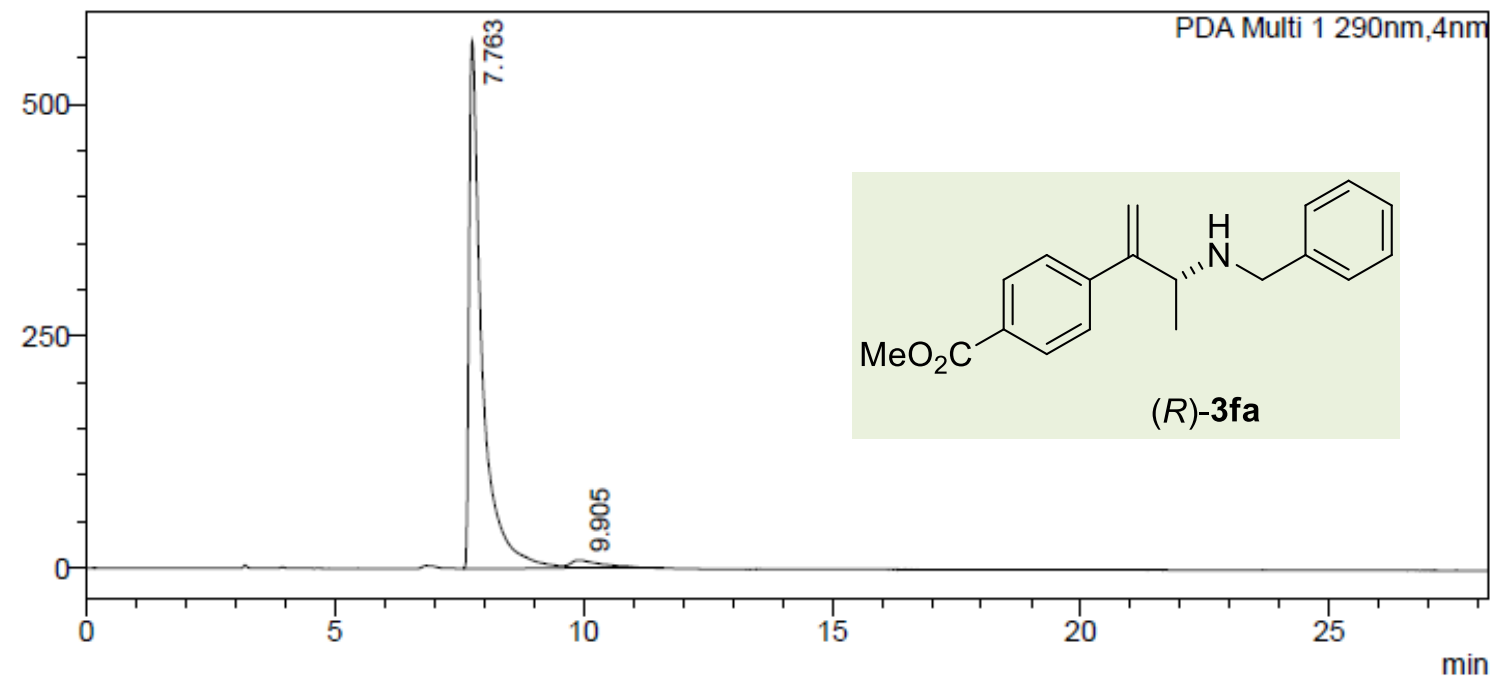

<Peak Table>

PDA Ch1 290nm

\begin{tabular}{|r|r|r|}
\hline Peak\# & Ret. Time & \multicolumn{1}{c|}{ Area\% } \\
\hline 1 & 7.763 & 96.180 \\
\hline 2 & 9.905 & 3.820 \\
\hline Total & & 100.000 \\
\hline
\end{tabular}




\section{(R)-N-Benzyl-3-(3-methoxyphenyl)but-3-en-2-amine 3ga}

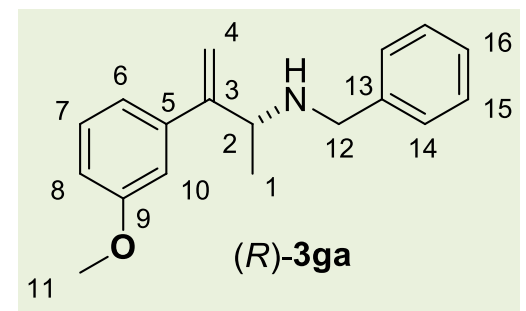

Synthesized at room temperature for $72 \mathrm{~h}$ following the general procedure using 1-(buta-1,3-dien-2-yl)-3methoxybenzene $\mathbf{1 g}(64.0 \mathrm{mg}, 0.40 \mathrm{mmol}, 1.0$ equiv), benzylamine $2 \mathbf{a}\left(175 \mu \mathrm{L}, 1.60 \mathrm{mmol}, 4.0\right.$ equiv), $\mathrm{Ni}(\operatorname{cod})_{2}(5.5$ $\mathrm{mg}, 0.020 \mathrm{mmol}, 5 \mathrm{~mol} \%),(R, R)-B^{2} \mathrm{BnP}^{*}(6.8 \mathrm{mg}, 0.024$ mmol, 6 mol\%), 2,2,2-trifluoroethanol ( $29 \mu \mathrm{L}, 0.40 \mathrm{mmol}, 1.0$ equiv) and mesitylene $(0.80 \mathrm{~mL}$, $0.5 \mathrm{M}$ ). Consumption of $\mathbf{1 g}>95 \%$, conversion of $3 \mathrm{ga}: 88 \%$, r.r. $=7: 1$. Purification by flash chromatography over silica gel (pentane:EtOAc $=3: 2$ ) led to the desired product as a pale yellow oil ( $88.0 \mathrm{mg}, 82 \%$ yield, $94 \%$ ee).

${ }^{1} \mathrm{H}$ NMR $\left(500 \mathrm{MHz}, \mathrm{CDCl}_{3}\right) \delta(\mathrm{ppm})=7.34-7.30(\mathrm{~m}, 4 \mathrm{H}, \mathrm{H}-14+\mathrm{H}-15), 7.26-7.21(\mathrm{~m}, 2 \mathrm{H}$, $\mathrm{H}-7+\mathrm{H}-16), 6.98-6.95(\mathrm{~m}, 1 \mathrm{H}, \mathrm{H}-6), 6.95-6.93(\mathrm{~m}, 1 \mathrm{H}, \mathrm{H}-10), 6.84$ (ddd, ${ }^{3} \mathrm{JHH}_{\mathrm{HH}}=8.1,{ }^{4} \mathrm{JHH}$ $=2.5,0.8 \mathrm{~Hz}, 1 \mathrm{H}, \mathrm{H}-8), 5.32(\mathrm{~s}, 2 \mathrm{H}, \mathrm{H}-4), 3.87\left(\mathrm{~d},{ }^{2} \mathrm{JH}=13.0 \mathrm{~Hz}, 1 \mathrm{H}, \mathrm{H}-12\right), 3.80(\mathrm{~s}, 3 \mathrm{H}, \mathrm{H}-$ 11), $3.76\left(\mathrm{~d},{ }^{2} \mathrm{~J}_{\mathrm{HH}}=13.0 \mathrm{~Hz}, 1 \mathrm{H}, \mathrm{H}-12\right), 3.72\left(\mathrm{q},{ }^{3} \mathrm{~J}_{\mathrm{HH}}=6.6 \mathrm{~Hz}, 1 \mathrm{H}, \mathrm{H}-2\right), 1.54(\mathrm{~s}, 1 \mathrm{H}, \mathrm{N}-H)$, $1.23\left(\mathrm{~d},{ }^{3} \mathrm{JHH}=6.6 \mathrm{~Hz}, 3 \mathrm{H}, \mathrm{H}-1\right)$.

${ }^{13} \mathrm{C}\left\{{ }^{1} \mathrm{H}\right\}$ NMR $\left(130 \mathrm{MHz}, \mathrm{CDCl}_{3}\right) \delta(\mathrm{ppm})=159.6(C-9), 151.9(C-3), 142.9(C-5), 140.8(C-$ 13), $129.3(\mathrm{CH}-7), 128.5(\mathrm{CH}-A r), 128.3(\mathrm{CH}-A r), 127.0(\mathrm{CH}-16), 119.7\left(\mathrm{CH}_{2}-4\right), 113.04(\mathrm{CH}-$ Ar), $112.97(\mathrm{CH}-\mathrm{Ar}), 112.8(\mathrm{CH}-\mathrm{Ar}), 56.8(\mathrm{CH}-2), 55.4\left(\mathrm{CH}_{3}-11\right), 51.4\left(\mathrm{CH}_{2}-12\right), 21.7\left(\mathrm{CH}_{3}-1\right)$. HRMS $\left(\mathrm{ESI}^{+}\right)$: calculated $[\mathrm{M}+\mathrm{H}]^{+}$for $\mathrm{C}_{18} \mathrm{H}_{22} \mathrm{NO}^{+}:$268.1696; found: 268.1731.

IR (neat) $\vee\left(\mathrm{cm}^{-1}\right): 3064,2962,2833,1597,1575,1487,1453,1428,1369,1315,1285,1221$, 1145, 1045, 907, 784, 730, 696.

HPLC: $94 \%$ ee, chiral stationary column: OJ-H, mobile phase: hexane/ $\mathrm{PrOH}=99.5 / 0.05$, $0.50 \mathrm{~mL} / \mathrm{min}, 254 \mathrm{~nm}, 30^{\circ} \mathrm{C}, \mathrm{t}_{\mathrm{R}}$ (major) $=37.0 \mathrm{~min}, \mathrm{t}_{\mathrm{R}}$ (minor) $=42.1 \mathrm{~min}$.

$[\alpha]^{20} \mathrm{D}=+2.1\left(\mathrm{c} 0.84, \mathrm{CHCl}_{3}\right)$. 


\section{<Chromatogram>}

mAU

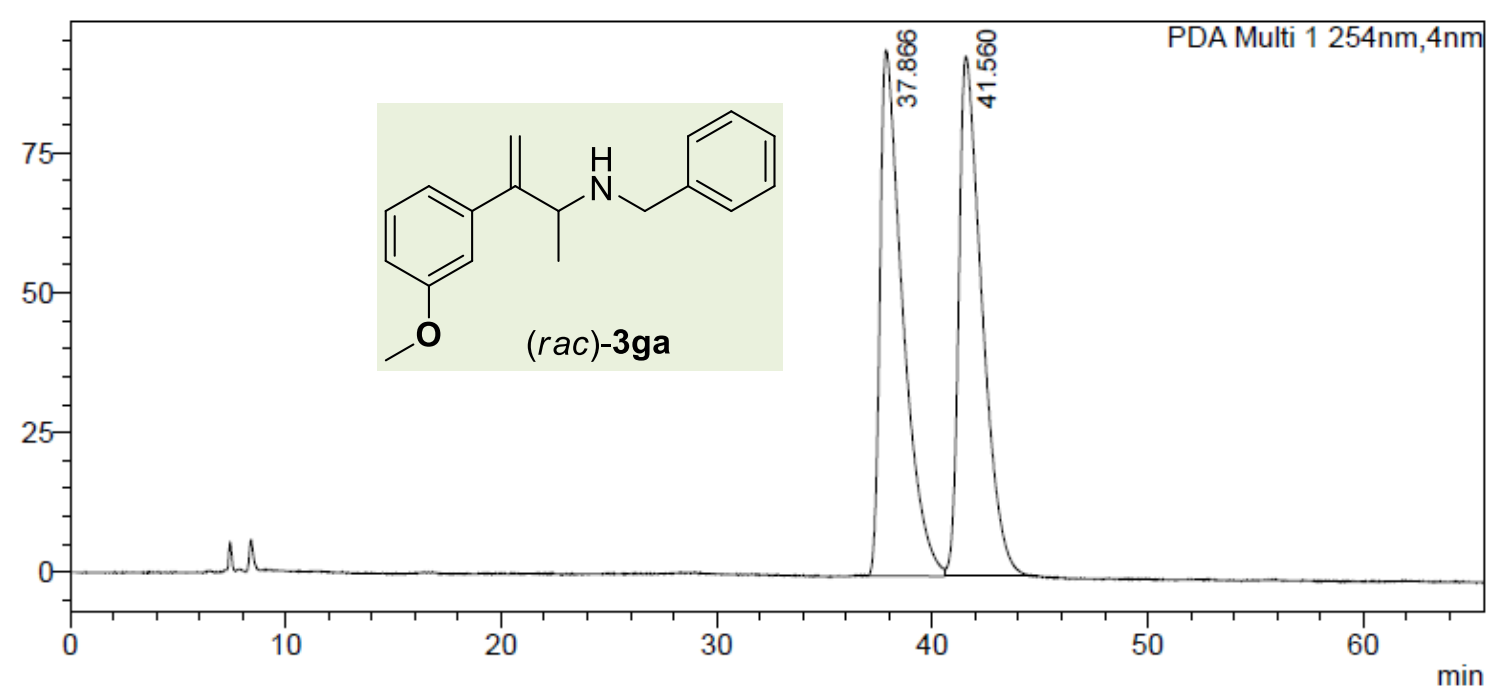

PDA Ch1 254nm

\begin{tabular}{|r|r|r|}
\hline Peak\# & Ret. Time & Area\% \\
\hline 1 & 37.866 & 49.795 \\
\hline 2 & 41.560 & 50.205 \\
\hline Total & & 100.000 \\
\hline
\end{tabular}

\section{$<$ Chromatogram>}

mAU

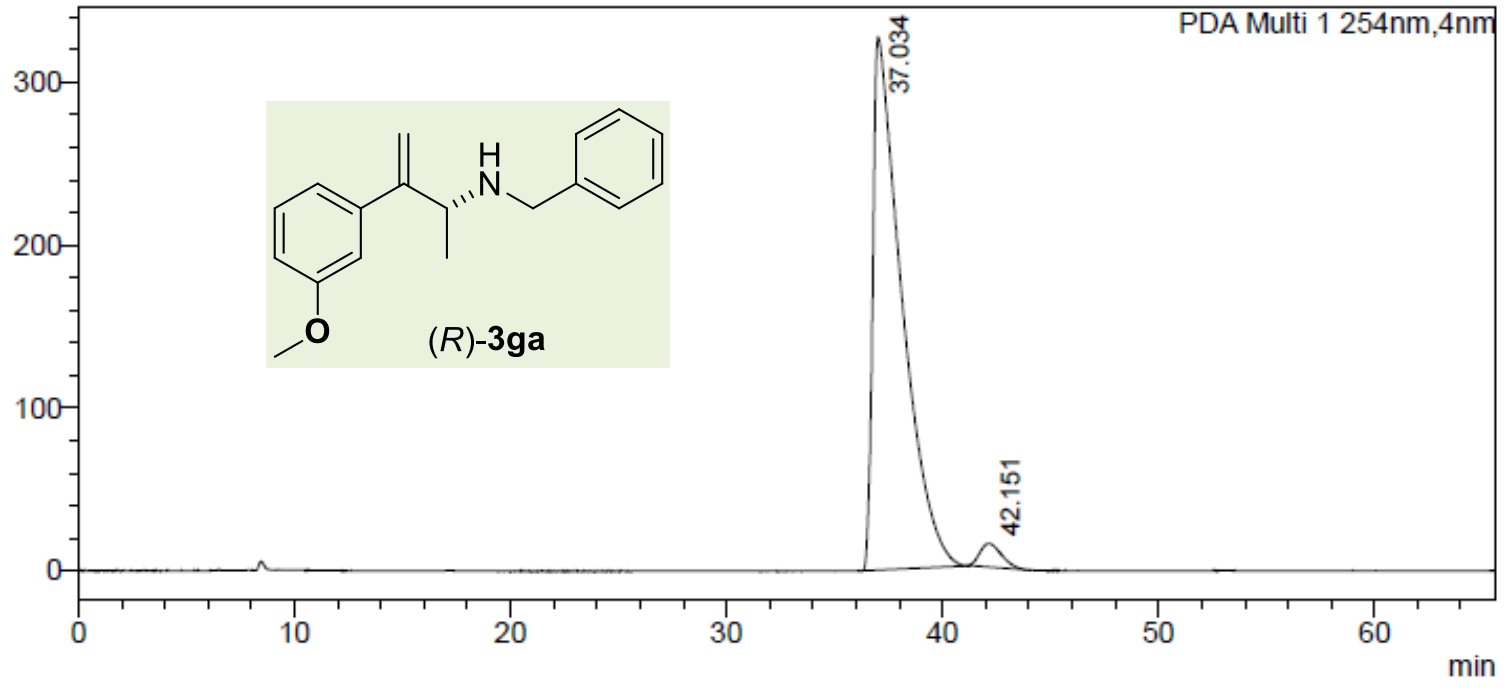

<Peak Table>

PDA Ch1 254nm

\begin{tabular}{|r|r|r|}
\hline Peak\# & Ret. Time & \multicolumn{1}{c|}{ Area\% } \\
\hline 1 & 37.034 & 96.797 \\
\hline 2 & 42.151 & 3.203 \\
\hline Total & & 100.000 \\
\hline
\end{tabular}




\section{(R)-N-Benzyl-3-(6-methoxypyridin-3-yl)but-3-en-2-amine 3ha}

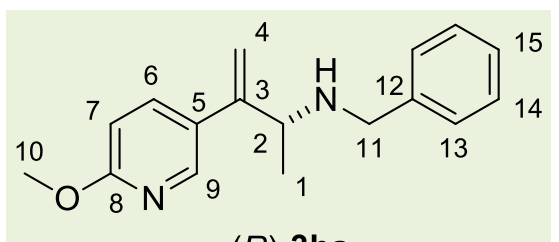

$(R)-3$ ha

Synthesized at room temperature for $72 \mathrm{~h}$ following the general procedure using 5-(buta-1,3-dien-2-yl)-2methoxypyridine $1 \mathrm{~h}$ (65.0 $\mathrm{mg}, 0.40 \mathrm{mmol}, 1.0$ equiv), benzylamine $2 \mathrm{a}\left(175 \mu \mathrm{L}, 1.60 \mathrm{mmol}, 4.0\right.$ equiv), $\mathrm{Ni}(\mathrm{cod})_{2}$ (5.5 mg, $0.020 \mathrm{mmol}, 5 \mathrm{~mol} \%),(R, R)-\operatorname{BenzP}^{*}(6.8 \mathrm{mg}$, $0.024 \mathrm{mmol}, 6 \mathrm{~mol} \%)$, 2,2,2-trifluoroethanol (29 $\mu \mathrm{L}, 0.40 \mathrm{mmol}, 1.0$ equiv) and mesitylene $(0.80 \mathrm{~mL}, 0.5 \mathrm{M})$. Consumption of $\mathbf{1 h}:>95 \%$, conversion of 3 ha: $80 \%$, r.r. $=5: 1$. Purification by flash chromatography over silica gel (pentane:EtOAc $=3: 2$ ) led to the desired product as a clear colorless oil $(84.0 \mathrm{mg}, 78 \%$ yield, $95 \%$ ee).

${ }^{1} \mathrm{H}$ NMR $\left(500 \mathrm{MHz}, \mathrm{CDCl}_{3}\right) \delta(\mathrm{ppm})=8.22\left(\mathrm{~d},{ }^{4} \mathrm{~J}_{\mathrm{HH}}=2.1 \mathrm{~Hz}, 1 \mathrm{H}, \mathrm{H}-9\right), 7.65\left(\mathrm{dd},{ }^{3} \mathrm{JHH}_{\mathrm{HH}}=8.7\right.$, $\left.{ }^{4} J_{\mathrm{HH}}=2.5 \mathrm{~Hz}, 1 \mathrm{H}, \mathrm{H}-6\right), 7.36-7.28(\mathrm{~m}, 4 \mathrm{H}, \mathrm{H}-13+\mathrm{H}-14), 7.29-7.22(\mathrm{~m}, 1 \mathrm{H}, \mathrm{H}-15), 6.70$ $\left(\mathrm{dd},{ }^{3} \mathrm{~J}_{\mathrm{HH}}=8.7,{ }^{5} \mathrm{~J}_{\mathrm{HH}}=0.6 \mathrm{~Hz}, 1 \mathrm{H}, \mathrm{H}-7\right), 5.30(\mathrm{~s}, 1 \mathrm{H}, \mathrm{H}-4), 5.26\left(\mathrm{~d},{ }^{2} J_{\mathrm{HH}}=1.2 \mathrm{~Hz}, 1 \mathrm{H}, \mathrm{H}-4\right)$, $3.94(\mathrm{~s}, 3 \mathrm{H}, \mathrm{H}-10), 3.86\left(\mathrm{~d},{ }^{2} J_{\mathrm{HH}}=13.2 \mathrm{~Hz}, 1 \mathrm{H}, \mathrm{H}-11\right), 3.74\left(\mathrm{~d},{ }^{2} J_{\mathrm{HH}}=13.2 \mathrm{~Hz}, 1 \mathrm{H}, \mathrm{H}-11\right)$, $3.67\left(\mathrm{q},{ }^{3} \mathrm{~J}_{\mathrm{HH}}=6.3 \mathrm{~Hz}, 1 \mathrm{H}, \mathrm{H}-2\right), 1.48(\mathrm{~s}, 1 \mathrm{H}, \mathrm{N}-H), 1.21\left(\mathrm{~d},{ }^{3} \mathrm{~J}_{\mathrm{HH}}=6.6 \mathrm{~Hz}, 3 \mathrm{H}, \mathrm{H}-1\right)$.

${ }^{13} \mathrm{C}\left\{{ }^{1} \mathrm{H}\right\}$ NMR $\left(130 \mathrm{MHz}, \mathrm{CDCl}_{3}\right) \delta(\mathrm{ppm})=163.7(C-8), 148.5(C-3), 145.2(\mathrm{CH}-9), 140.7$ (C-12), $137.7(\mathrm{CH}-6), 129.8(\mathrm{C}-5), 128.6(\mathrm{CH}-\mathrm{Ar}), 128.3(\mathrm{CH}-\mathrm{Ar}), 127.1(\mathrm{CH}-15), 113.4\left(\mathrm{CH}_{2}-\right.$ 4), $110.3(\mathrm{CH}-7), 57.2(\mathrm{CH}-2), 53.6\left(\mathrm{CH}_{3}-10\right), 51.5\left(\mathrm{CH}_{2}-11\right), 21.6\left(\mathrm{CH}_{3}-1\right)$.

HRMS (ESI+): calculated [M+H] for $\mathrm{C}_{17} \mathrm{H}_{20} \mathrm{~N}_{2} \mathrm{O}^{+}$: 269.1649; found: 269.1689.

IR (neat) $\vee\left(\mathrm{cm}^{-1}\right): 3027,2971,624,1599,1490,1455,1366,1282,1248,1126,1024,907$, 832, 734, 697.

HPLC: $95 \%$ ee, chiral stationary column: $\mathrm{OJ}-\mathrm{H}$, mobile phase: hexane/ $\mathrm{PrOH}=99.5 / 0.05$, $0.50 \mathrm{~mL} / \mathrm{min}, 254 \mathrm{~nm}, 30^{\circ} \mathrm{C}, \mathrm{t}_{\mathrm{R}}$ (major) $=40.8 \mathrm{~min}, \mathrm{t}_{\mathrm{R}}$ (minor) $=44.8 \mathrm{~min}$.

$[\alpha]^{20} \mathrm{D}=+16.7\left(c 1.01, \mathrm{CHCl}_{3}\right)$. 


\section{<Cnromatogram>}

mAU

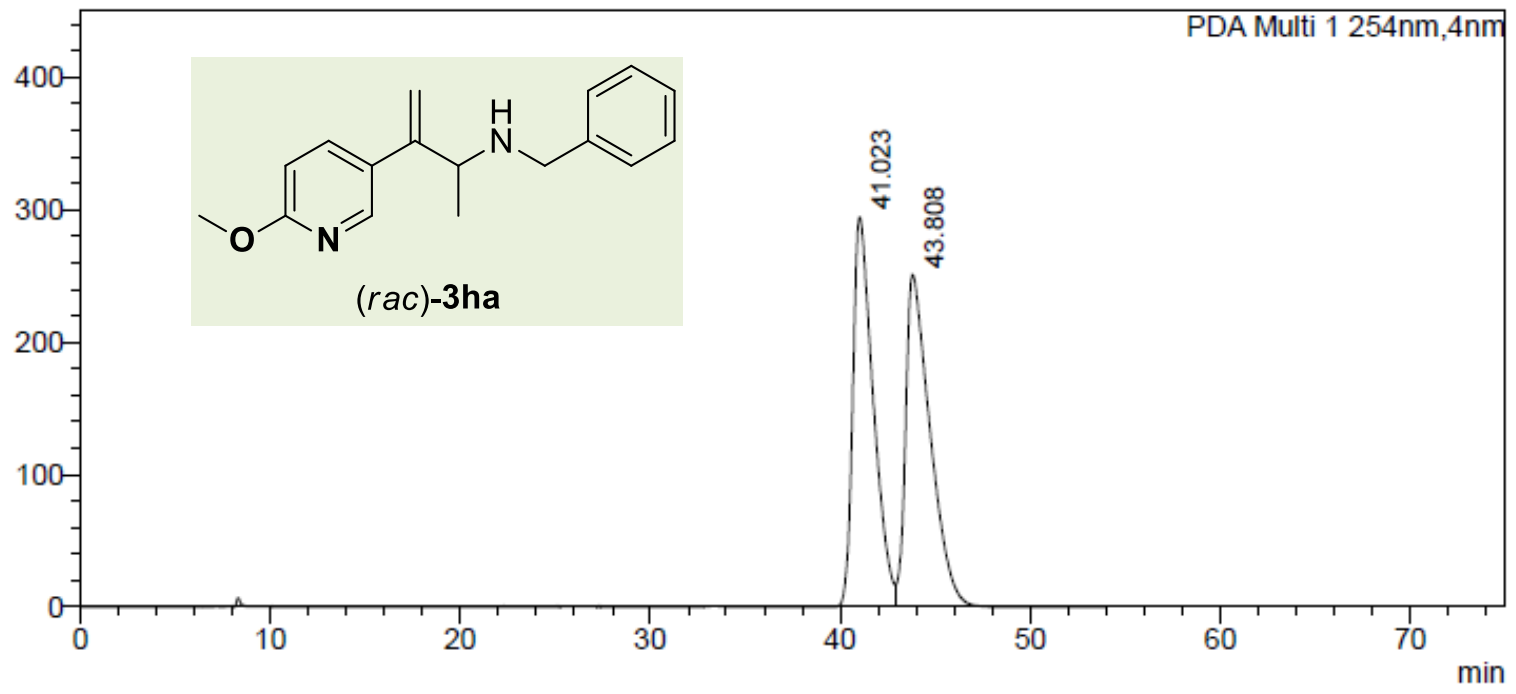

<Peak Table>

PDA Ch1 254nm

\begin{tabular}{|r|r|r|}
\hline Peak\# & Ret. Time & \multicolumn{1}{|c|}{ Area\% } \\
\hline 1 & 41.023 & 49.349 \\
\hline 2 & 43.808 & 50.651 \\
\hline Total & & 100.000 \\
\hline
\end{tabular}

<Chromatogram>

mAU

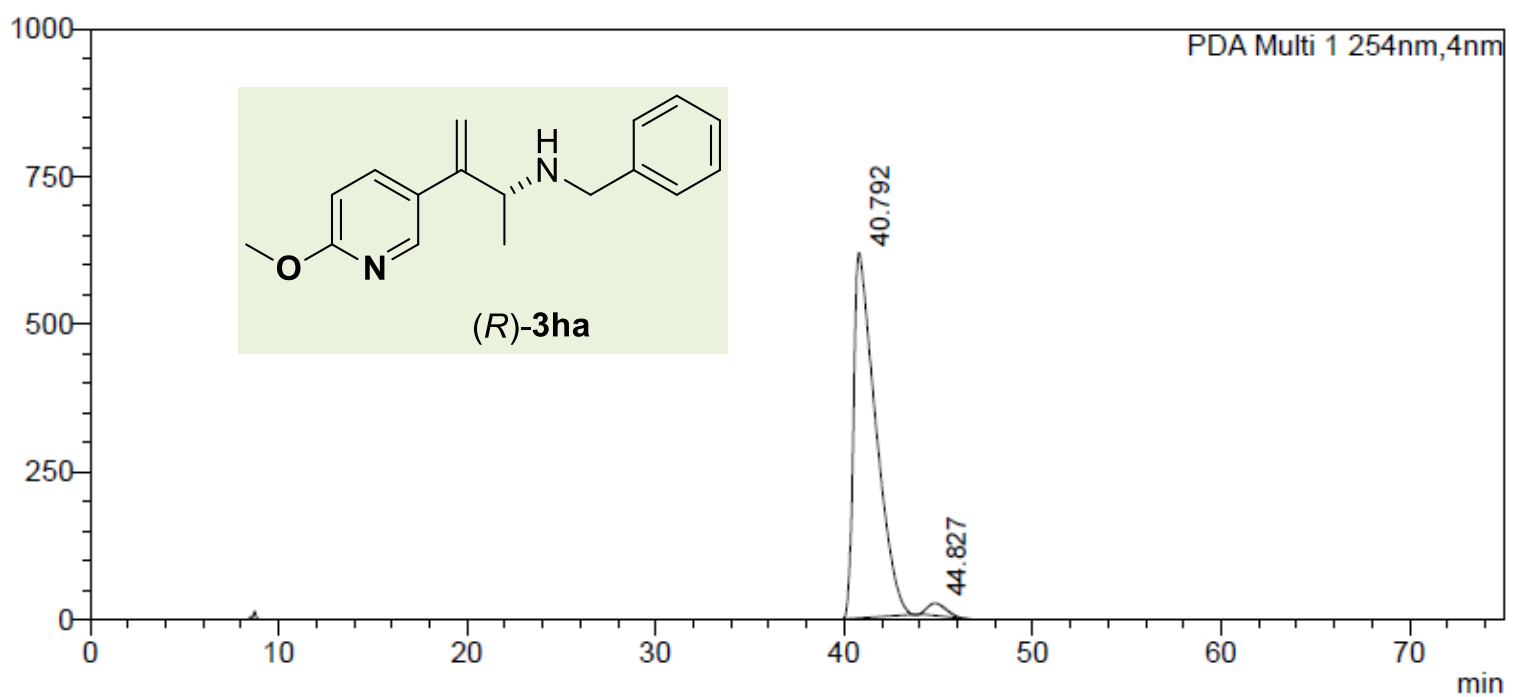

<Peak Table>

PDA Ch1 254nm

\begin{tabular}{|r|r|r|}
\hline Peak\# & Ret. Time & \multicolumn{1}{c|}{ Area $\%$} \\
\hline 1 & 40.792 & 97.244 \\
\hline 2 & 44.827 & 2.756 \\
\hline Total & & 100.000 \\
\hline
\end{tabular}




\section{(R)-N-Benzyl-3-methylene-5-phenylpentan-2-amine 3ia}

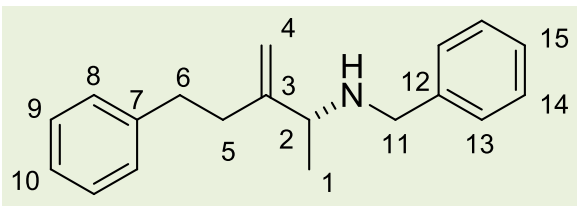

$(R)-3 \mathbf{i a}$

Synthesized at room temperature for $72 \mathrm{~h}$ following the general procedure using (3-methylenepent-4-en-1yl)benzene 1i (40.0 mg, 0.25 mmol, 1.0 equiv), benzylamine $2 \mathrm{a}\left(110 \mu \mathrm{L}, 1.00 \mathrm{mmol}, 4.0\right.$ equiv), $\mathrm{Ni}(\mathrm{cod})_{2}$ (3.4 mg, $0.0125 \mathrm{mmol}, 5 \mathrm{~mol} \%),(R, R)$-BenzP* (4.2 mg, $0.015 \mathrm{mmol}, 6 \mathrm{~mol} \%), \mathrm{Ph}_{2} \mathrm{P}(\mathrm{O}) \mathrm{OH}$ (55.0 mg, $0.25 \mathrm{mmol}, 1.0$ equiv) and mesitylene ( $0.50 \mathrm{~mL}$, $0.5 \mathrm{M}$ ). Consumption of $1 \mathrm{i}: 93 \%$, conversion of 3ia: $48 \%$, r.r. $=1.1: 1$. Purification by flash chromatography over silica gel (pentane:EtOAc $=4: 1$ ) led to the desired product as a pale yellow oil ( $30.0 \mathrm{mg}, 45 \%$ yield, $15 \%$ ee).

${ }^{1} \mathrm{H}$ NMR $\left(300 \mathrm{MHz}, \mathrm{CDCl}_{3}\right) \delta(\mathrm{ppm})=7.29-7.13(\mathrm{~m}, 10 \mathrm{H}, \mathrm{H}-A r), 5.01(\mathrm{~s}, 1 \mathrm{H}, \mathrm{H}-4), 4.90(\mathrm{q}$, $\left.{ }^{2} J_{H H}=1.4 \mathrm{~Hz}, 1 \mathrm{H}, \mathrm{H}-4\right), 3.67\left(\mathrm{~d},{ }^{2} J_{\mathrm{HH}}=13.1 \mathrm{~Hz}, 1 \mathrm{H}, \mathrm{H}-11\right), 3.56\left(\mathrm{~d},{ }^{2} J_{\mathrm{HH}}=13.1 \mathrm{~Hz}, 1 \mathrm{H}\right.$, $\mathrm{H}-11$ ), 3.23 (q, $\left.{ }^{3} \mathrm{JHH}_{\mathrm{H}}=6.6 \mathrm{~Hz}, 1 \mathrm{H}, \mathrm{H}-2\right), 2.85-2.67(\mathrm{~m}, 2 \mathrm{H}, \mathrm{H}-5), 2.43-2.21(\mathrm{~m}, 2 \mathrm{H}, \mathrm{H}-6)$, $1.26(\mathrm{~s}, 1 \mathrm{H}, \mathrm{N}-\mathrm{H}), 1.14\left(\mathrm{~d},{ }^{3} \mathrm{JHH}_{\mathrm{H}}=6.6 \mathrm{~Hz}, 3 \mathrm{H}, \mathrm{H}-1\right)$.

${ }^{13} \mathrm{C}\left\{{ }^{1} \mathrm{H}\right\}$ NMR $\left(75 \mathrm{MHz}, \mathrm{CDCl}_{3}\right) \delta(\mathrm{ppm})=151.7(C-3), 142.5(C-7), 141.0(C-12), 128.52$ $(\mathrm{CH}-A r), 128.48(\mathrm{CH}-\mathrm{Ar}), 128.46(\mathrm{CH}-\mathrm{Ar}), 128.3(\mathrm{CH}-\mathrm{Ar}), 126.9(\mathrm{CH}-10$ or $\mathrm{CH}-15), 125.9$ $(\mathrm{CH}-10$ or $\mathrm{CH}-15), 110.0\left(\mathrm{CH}_{2}-4\right), 58.8(\mathrm{CH}-2), 51.6\left(\mathrm{CH}_{2}-11\right), 34.7\left(\mathrm{CH}_{2}-6\right), 33.0\left(\mathrm{CH}_{2}-5\right)$, $21.4\left(\mathrm{CH}_{3}-1\right)$.

HRMS (ESI+): calculated $[\mathrm{M}+\mathrm{H}]^{+}$for $\mathrm{C}_{19} \mathrm{H}_{24} \mathrm{~N}^{+}: 266.1914$; found: 266.1937 .

IR (neat) $\vee\left(\mathrm{cm}^{-1}\right): 3027,2925,1643,1604,1495,1453,1369,1317,1202,1120,1029,897$, $738,695$.

HPLC: $15 \%$ ee, chiral stationary column: $\mathrm{OJ}-\mathrm{H}$, mobile phase: hexane/iPrOH $=99 / 1,1.0$ $\mathrm{mL} / \mathrm{min}, 254 \mathrm{~nm}, 30^{\circ} \mathrm{C}, \mathrm{t}_{\mathrm{R}}$ (major) $=13.2 \mathrm{~min}, \mathrm{t}_{\mathrm{R}}($ minor $)=14.7 \mathrm{~min}$.

$[\alpha]^{20} \mathrm{D}=+1.8\left(c 0.355, \mathrm{CH}_{2} \mathrm{Cl}_{2}\right)$. 
$<$ Chromatogram>

mAU

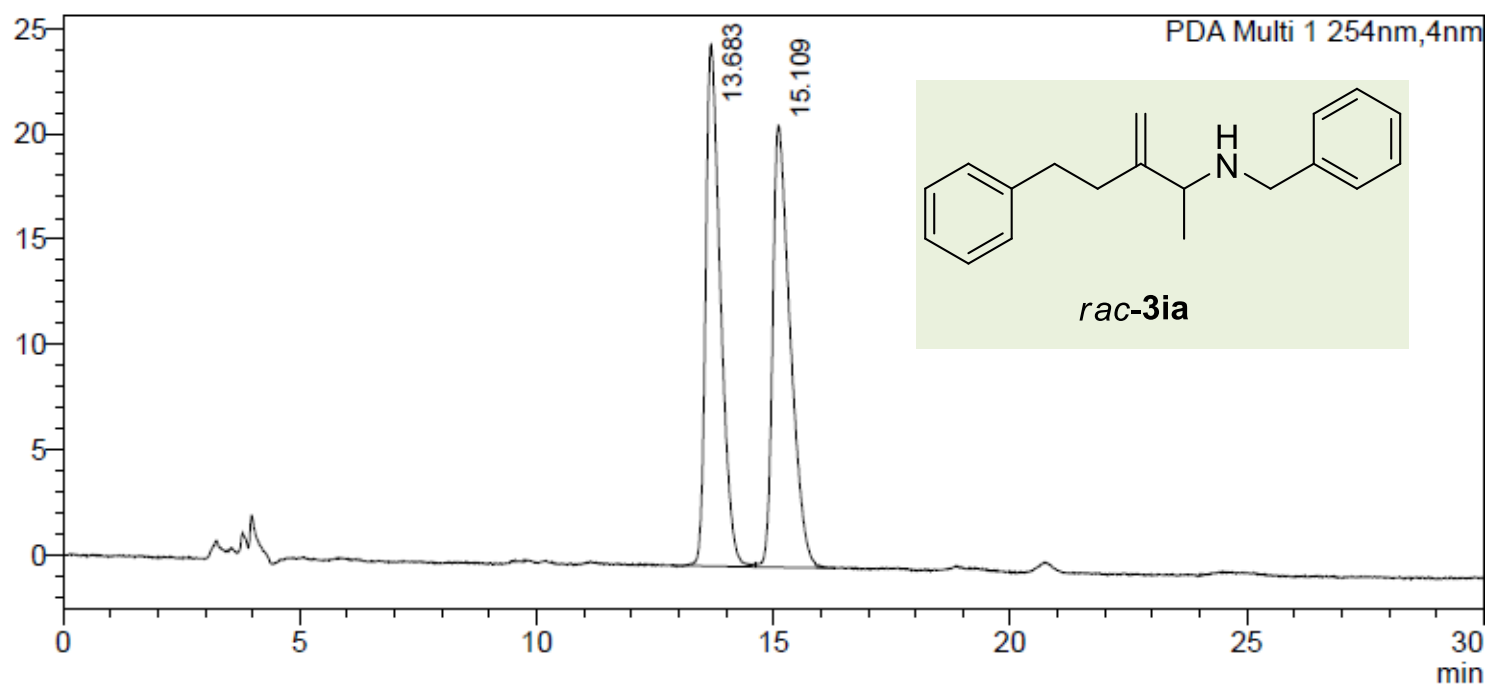

<Peak Table>

PDA Ch1 254nm \begin{tabular}{|r|r|}
\hline Peak\# & Ret. Time \\
\hline
\end{tabular}

\begin{tabular}{|r|r|r|}
\hline 1 & 13.683 & 49.778 \\
\hline 2 & 15.109 & 50.222 \\
\hline Total & & 100.000 \\
\hline
\end{tabular}

$<$ Chromatogram>

mAU

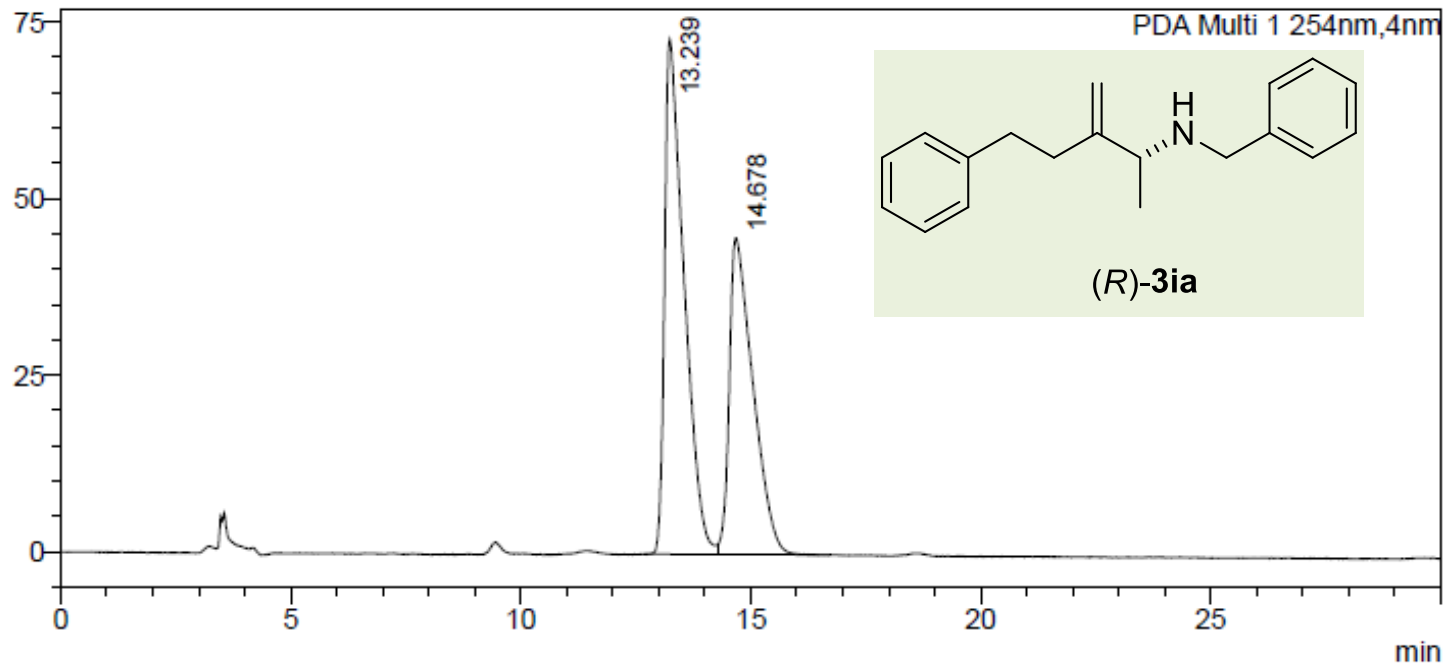

PDA Ch1 254nm

\begin{tabular}{|r|r|r|}
\hline Peak\# & Ret. Time & \multicolumn{1}{|c|}{ Area\% } \\
\hline 1 & 13.239 & 57.325 \\
\hline 2 & 14.678 & 42.675 \\
\hline Total & & 100.000 \\
\hline
\end{tabular}




\subsection{Scope in amines}

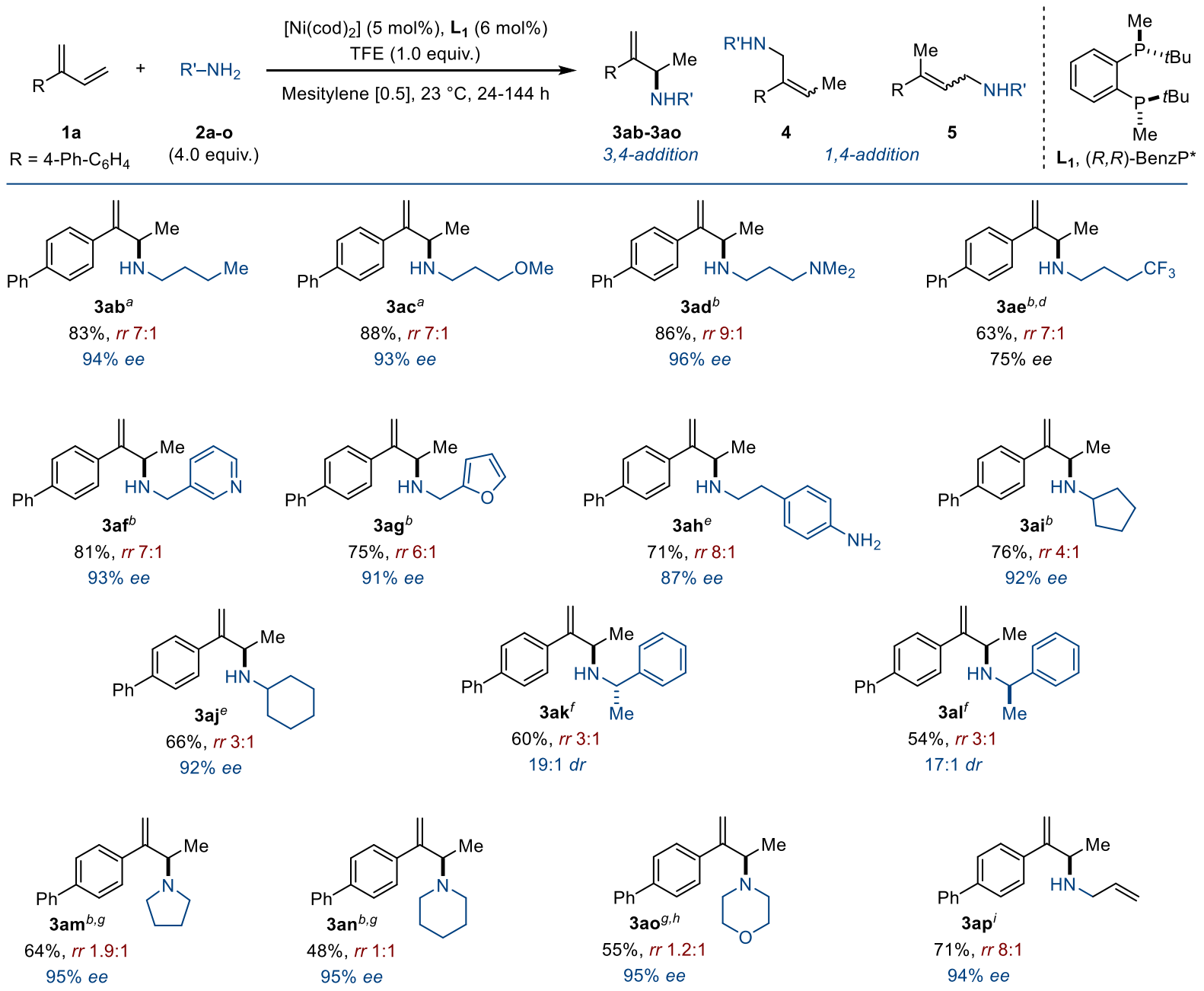

Figure S2. Scope of the Ni-catalyzed hydroamination of 2-substituted 1,3-dienes Variation of the amine component. $0.25-0.40 \mathrm{mmol}$ scale. Yields of 3,4-addition product after purification. Regioselectivity expressed as the ratio between 3,4- and 1,4-addition products as determined by ${ }^{1} \mathrm{H}$ NMR using an internal standard $(3:[4+5])$. Enantiomeric excess determined by GC or HPLC equipped with chiral columns. ${ }^{a} 48$ h. ${ }^{b} 72$ h. ${ }^{c} 120$ h. ${ }^{d} 40$ ${ }^{\circ} \mathrm{C} .{ }^{e} 96$ h. ${ }^{f} 144$ h. ${ }^{g} 0.2$ equiv. $\mathrm{Ph}_{2} \mathrm{P}(\mathrm{O}) \mathrm{OH}$ instead of TFE. ${ }^{h} 24 \mathrm{~h} .{ }^{i} 20 \mathrm{~h}$ 


\section{(R)-3-([1,1'-Biphenyl]-4-yl)-N-butylbut-3-en-2-amine 3ab}

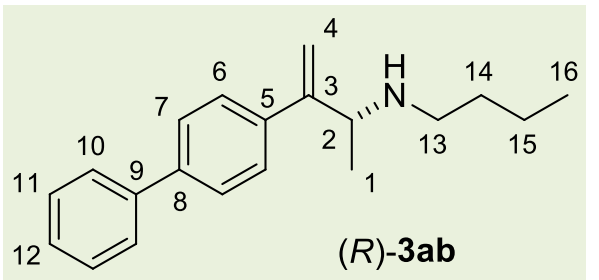

Synthesized at room temperature for $48 \mathrm{~h}$ in $0.40 \mathrm{mmol}$

scale following the general procedure using 4-(buta-1,3dien-2-yl)-1,1'-biphenyl 1a (82.5 mg, $0.40 \mathrm{mmol}, 1.0$ equiv), $n$-butylamine $\mathbf{2 b}(158 \mu \mathrm{L}, 1.60 \mathrm{mmol}, 4.0$ equiv), $\mathrm{Ni}(\operatorname{cod})_{2}(5.5 \mathrm{mg}, 0.020 \mathrm{mmol}, 5 \mathrm{~mol} \%),(R, R)-B e n z \mathrm{P}^{*}$ (6.8 mg, $0.024 \mathrm{mmol}, 6 \mathrm{~mol} \%$ ), 2,2,2-trifluoroethanol (30 $\mu \mathrm{L}, 0.40 \mathrm{mmol}, 1.0$ equiv) and mesitylene $(0.80 \mathrm{~mL}, 0.5 \mathrm{M})$. Consumption of 1a: $>99 \%$, conversion of 3ab: $87 \%$, r.r. $=7: 1$. Purification by flash chromatography over silica gel (pentane:EtOAc $=3: 1$ ) led to the desired product as a colorless viscous oil ( $93.0 \mathrm{mg}, 83 \%$ yield, $94 \%$ ee).

${ }^{1} \mathrm{H}$ NMR $\left(400 \mathrm{MHz}, \mathrm{CDCl}_{3}\right) \delta(\mathrm{ppm})=7.64-7.54(\mathrm{~m}, 4 \mathrm{H}, \mathrm{H}-10+\mathrm{H}-7), 7.51-7.40(\mathrm{~m}, 4 \mathrm{H}, \mathrm{H}-$ $6+H-11), 7.39-7.30(\mathrm{~m}, 1 \mathrm{H}, H-12), 5.33(\mathrm{~s}, 1 \mathrm{H}, H-4), 5.29(\mathrm{~s}, 1 \mathrm{H}, H-4), 3.74\left(\mathrm{q},{ }^{3} J_{\mathrm{HH}}=6.5\right.$ $\mathrm{Hz}, 1 \mathrm{H}, H-2), 2.77-2.53(\mathrm{~m}, 2 \mathrm{H}, H-13), 1.54-1.46(\mathrm{~m}, 2 \mathrm{H}, H-14), 1.41-1.22(\mathrm{~m}, 3 \mathrm{H}, H-$ $15+\mathrm{NH}), 1.25\left(\mathrm{~d},{ }^{3} \mathrm{~J}_{\mathrm{HH}}=6.5 \mathrm{~Hz}, 3 \mathrm{H}, H-1\right), 0.91\left(\mathrm{t},{ }^{3} J_{\mathrm{HH}}=7.3 \mathrm{~Hz}, 3 \mathrm{H}, H-16\right)$.

${ }^{13} \mathrm{C}\left\{{ }^{1} \mathrm{H}\right\}$ NMR $\left(100 \mathrm{MHz}, \mathrm{CDCl}_{3}\right) \delta(\mathrm{ppm})=151.9(C-3), 140.9(C-9), 140.4(C-5), 140.3(C-8)$, $128.9(\mathrm{CH}-11), 127.5(\mathrm{CH}-6), 127.4(\mathrm{CH}-12), 127.13(\mathrm{CH}-10), 127.05(\mathrm{CH}-7), 112.5\left(\mathrm{CH}_{2}-4\right)$, $57.5(\mathrm{CH}-2), 47.4\left(\mathrm{CH}_{2}-13\right), 32.6\left(\mathrm{CH}_{2}-14\right), 21.8\left(\mathrm{CH}_{3}-1\right), 20.7\left(\mathrm{CH}_{2}-15\right), 14.2\left(\mathrm{CH}_{3}-16\right)$.

HRMS (ESI+): calculated [M+H] ${ }^{+}$for $\mathrm{C}_{20} \mathrm{H}_{26} \mathrm{~N}^{+}:$:280.2060; found: 280.2067.

IR (neat) $v\left(\mathrm{~cm}^{-1}\right):$ 3030, 2958, 2926, 2870, 1625, 1601, 1486, 1448, 1370, 1137, 1077, 1007, 907, 842, 769, 739, 695.

HPLC: $94 \%$ ee, chiral stationary column: $\mathrm{OJ}-\mathrm{H}$, mobile phase: hexane/iPrOH $=97 / 3,1.0$ $\mathrm{mL} / \mathrm{min}, 254 \mathrm{~nm}, 30^{\circ} \mathrm{C}, \mathrm{t}_{\mathrm{R}}($ minor $)=6.2 \mathrm{~min}, \mathrm{t}_{\mathrm{R}}($ major $)=7.5 \mathrm{~min}$.

$[\alpha]^{20} \mathrm{D}=-3.8\left(c \quad 0.1, \mathrm{CHCl}_{3}\right)$. 


\section{<Chromatogram >}

mAU

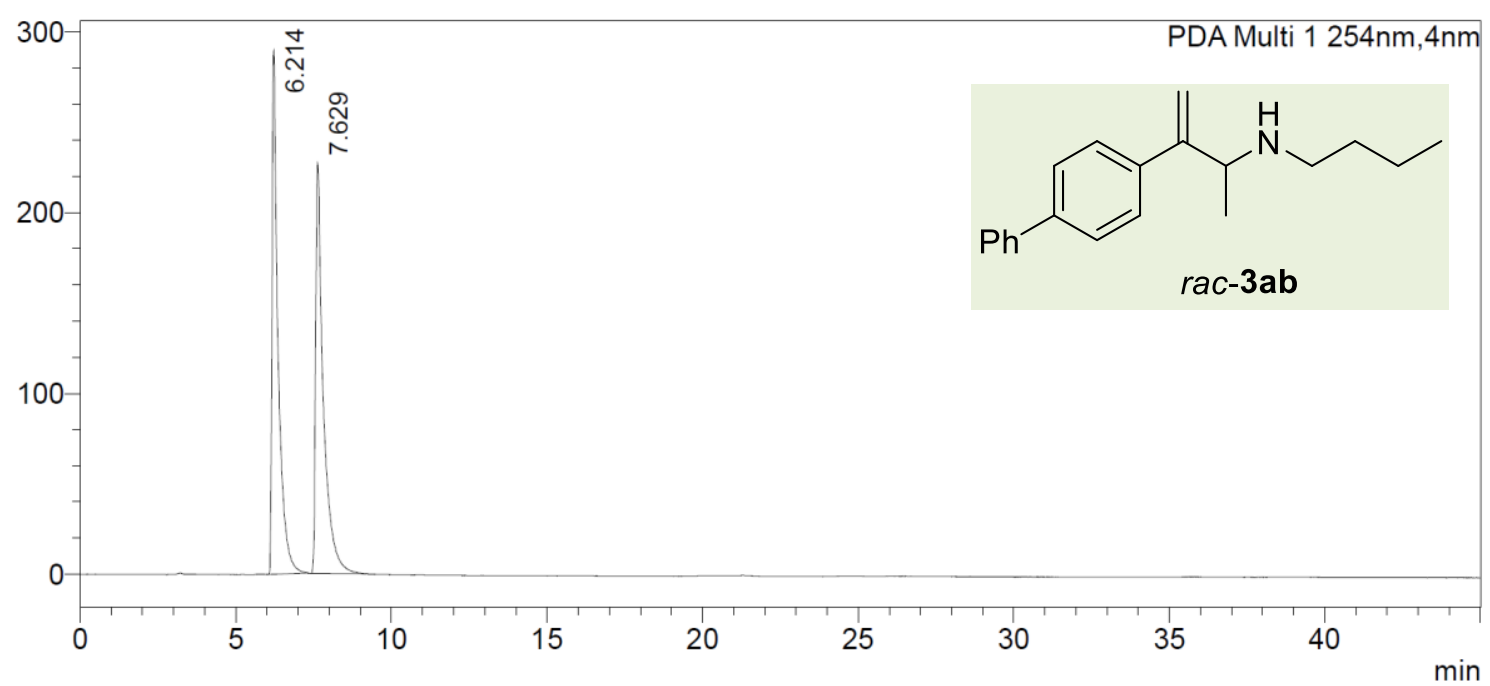

<Peak Table>

PDA Ch1 254nm

\begin{tabular}{|l|l}
\hline Peak\# Ret. Time & Area\% \\
\hline
\end{tabular}

\begin{tabular}{|r|r|r|}
\hline 1 & 6.214 & \multicolumn{1}{|c|}{ Area $\%$} \\
\hline 2 & 7.629 & 49.901 \\
\hline Total & & 100.000 \\
\hline
\end{tabular}

\section{$<$ Chromatogram $>$}

mAU

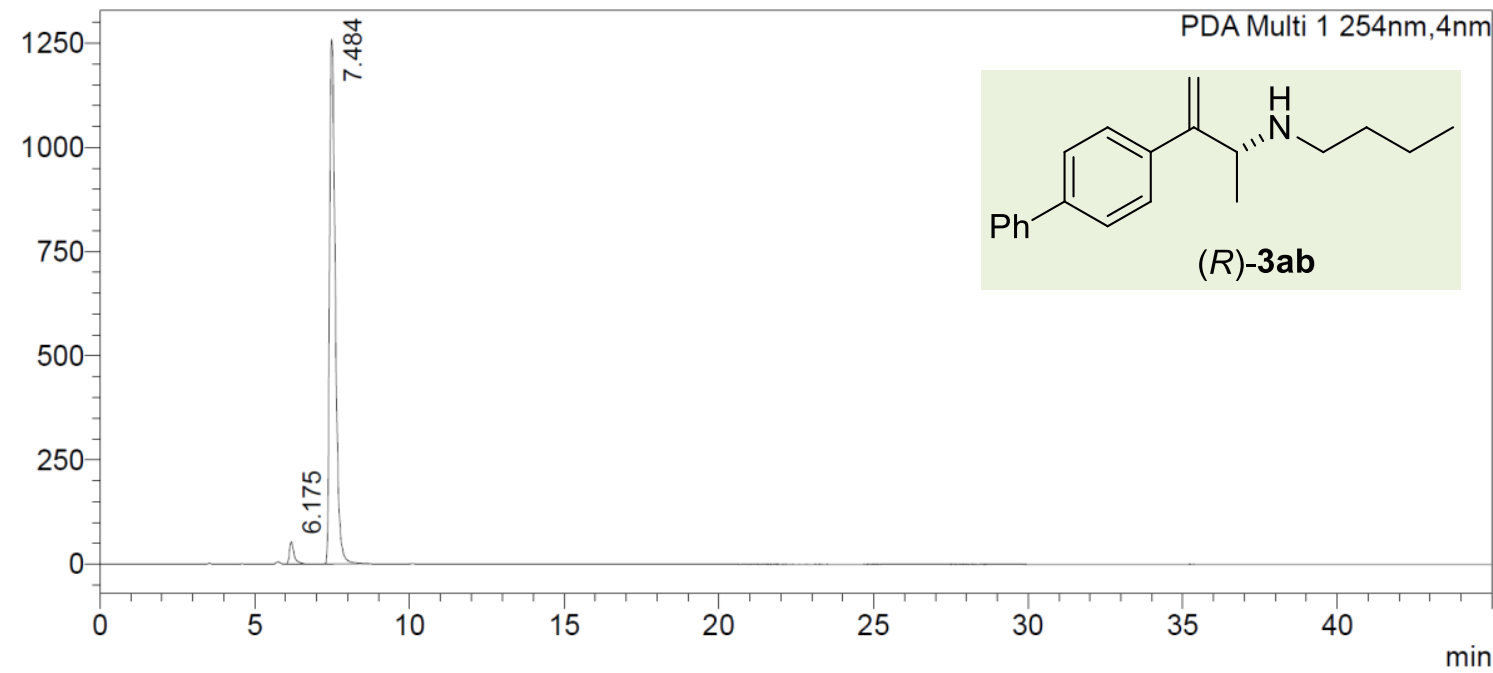

<Peak Table>

PDA Ch1 254nm

\begin{tabular}{|r|r|r|}
\hline Peak\# & Ret. Time & \multicolumn{1}{|c|}{ Area\% } \\
\hline 1 & 6.175 & 3.231 \\
\hline 2 & 7.484 & 96.769 \\
\hline Total & & 100.000 \\
\hline
\end{tabular}




\section{(R)-3-([1,1'-Biphenyl]-4-yl)-N-(3-methoxypropyl)but-3-en-2-amine 3ac}

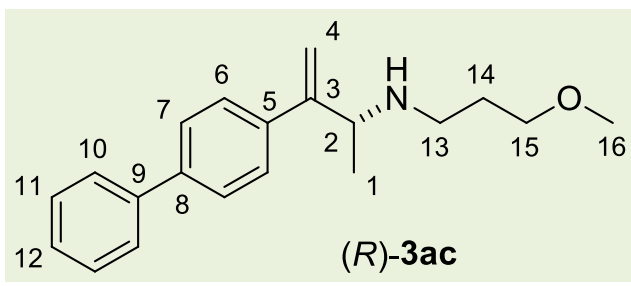

Synthesized at room temperature for $72 \mathrm{~h}$ following the general procedure using 4-(buta-1,3-dien-2-yl)1,1'-biphenyl 1a (51.6 mg, 0.25 mmol, 1.0 equiv), 3methoxypropylamine 2c $(102 \mu \mathrm{L}, 1.00 \mathrm{mmol}, 4.0$ equiv), $\mathrm{Ni}(\mathrm{cod})_{2}$ (3.4 mg, $\left.0.0125 \mathrm{mmol}, 5 \mathrm{~mol} \%\right)$, $(R, R)$-BenzP* (4.2 mg, $0.015 \mathrm{mmol}, 6 \mathrm{~mol} \%)$, 2,2,2-trifluoroethanol (19 $\mu \mathrm{L}, 0.25 \mathrm{mmol}, 1.0$ equiv) and mesitylene (0.50 mL, $0.5 \mathrm{M})$. Consumption of 1a: $>99 \%$, conversion of 3ac: $87 \%$, r.r. $=7: 1$. Purification by flash chromatography over silica gel $\left(\mathrm{CH}_{2} \mathrm{Cl}_{2}: \mathrm{MeOH}=20: 1\right)$ led to the desired product as a yellow viscous oil $(65.3 \mathrm{mg}, 88 \%$ yield, $93 \%$ ee).

${ }^{1} \mathrm{H}$ NMR $\left(400 \mathrm{MHz}, \mathrm{CDCl}_{3}\right) \delta(\mathrm{ppm})=7.64-7.54(\mathrm{~m}, 4 \mathrm{H}, \mathrm{H}-7+\mathrm{H}-10), 7.50-7.41(\mathrm{~m}, 4 \mathrm{H}, \mathrm{H}-$ $6+H-11), 7.38-7.31(\mathrm{~m}, 1 \mathrm{H}, H-12), 5.35\left(\mathrm{~d},{ }^{2} J_{H H}=1.0 \mathrm{~Hz}, 1 \mathrm{H}, H-4\right), 5.30(\mathrm{~s}, 1 \mathrm{H}, H-4), 3.78$ (q, $\left.{ }^{3} J_{H H}=6.5 \mathrm{~Hz}, 1 \mathrm{H}, H-2\right), 3.45\left(\mathrm{t},{ }^{3} \mathrm{~J}_{\mathrm{HH}}=6.2 \mathrm{~Hz}, 2 \mathrm{H}, H-15\right), 3.29(\mathrm{~s}, 3 \mathrm{H}, H-16), 2.87-2.69$ (m, 2H, H-13), $2.42(\mathrm{br}, 1 \mathrm{H}, \mathrm{NH}), 1.81\left(\mathrm{p},{ }^{3} \mathrm{~J}_{\mathrm{HH}}=6.5 \mathrm{~Hz}, 2 \mathrm{H}, \mathrm{H}-14\right), 1.28\left(\mathrm{~d},{ }^{3} \mathrm{~J}_{\mathrm{HH}}=6.6 \mathrm{~Hz}\right.$, $3 \mathrm{H}, \mathrm{H}-1)$.

${ }^{13} \mathrm{C}\left\{{ }^{1} \mathrm{H}\right\}$ NMR $\left(100 \mathrm{MHz}, \mathrm{CDCl}_{3}\right) \delta(\mathrm{ppm})=151.3(C-3), 140.9(C-8), 140.4(C-9), 140.2(C-5)$, $128.9(\mathrm{CH}-11), 127.5(\mathrm{CH}-7), 127.4(\mathrm{CH}-12), 127.12(\mathrm{CH}-6), 127.10(\mathrm{CH}-10), 113.0\left(\mathrm{CH}_{2}-4\right)$, $71.6\left(\mathrm{CH}_{2}-15\right), 58.8\left(\mathrm{CH}_{3}-16\right), 57.5(\mathrm{CH}-2), 44.9\left(\mathrm{CH}_{2}-13\right), 30.1\left(\mathrm{CH}_{2}-14\right), 21.4\left(\mathrm{CH}_{3}-1\right)$.

HRMS $\left(\mathrm{ESI}^{+}\right)$: calculated $[\mathrm{M}+\mathrm{H}]^{+}$for $\mathrm{C}_{20} \mathrm{H}_{26} \mathrm{NO}^{+}:$296.2009; found: 296.2069.

IR (neat) $v\left(\mathrm{~cm}^{-1}\right): 3030,2925,2867,1626,1598,1486,1448,1387,1191,1116,1077,1038$, 1006, 907, 844, 771, 741, 697.

HPLC: $93 \%$ ee, chiral stationary column: $\mathrm{OJ}-\mathrm{H}$, mobile phase: hexane/iPrOH $=95 / 5,1.0$ $\mathrm{mL} / \min , 254 \mathrm{~nm}, 30^{\circ} \mathrm{C}, \mathrm{t}_{\mathrm{R}}$ (minor) $=10.7 \mathrm{~min}, \mathrm{t}_{\mathrm{R}}($ major $)=13.5 \mathrm{~min}$.

$[\alpha]^{20} \mathrm{D}=+9.4\left(c 1.0, \mathrm{CHCl}_{3}\right)$. 
$<$ Chromatogram $>$

mAU

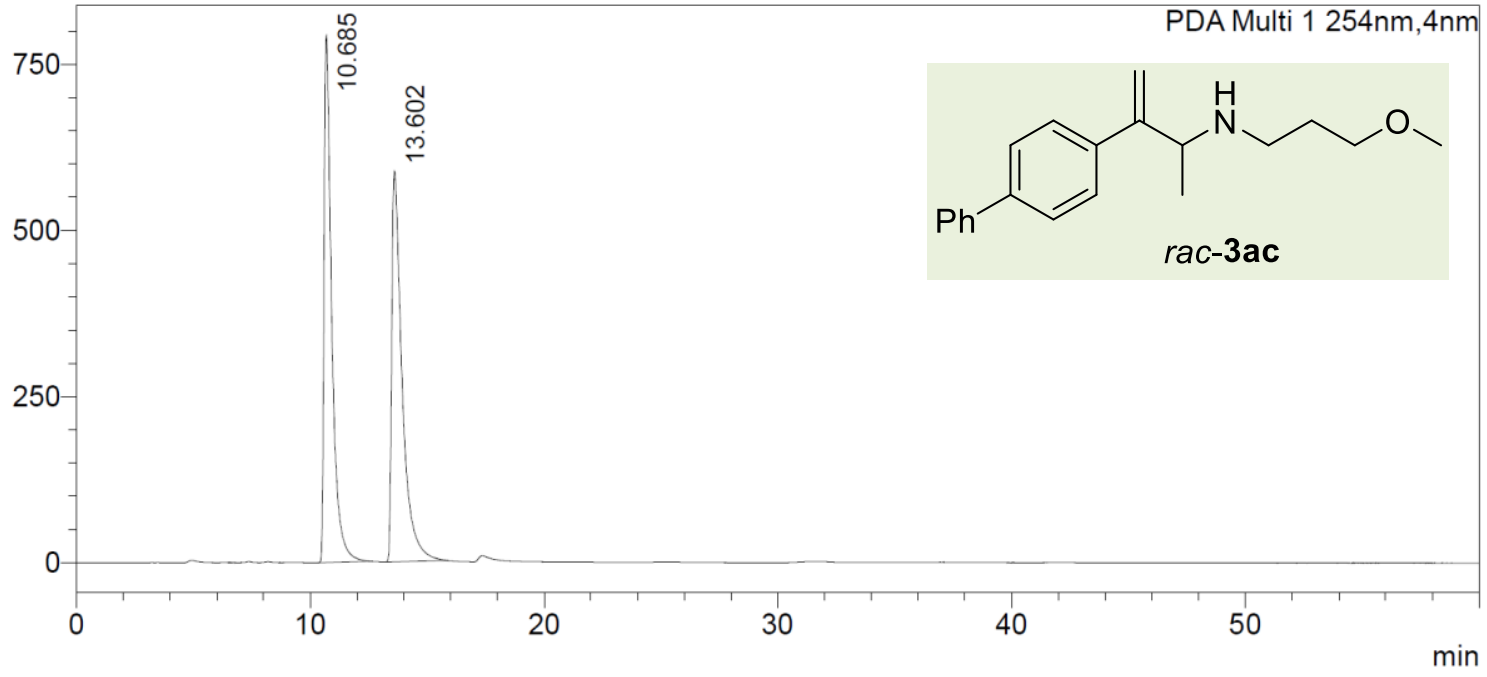

<Peak Table>

PDA Ch1 254nm

\begin{tabular}{|r|r|r|}
\hline Peak\# & Ret. Time & \multicolumn{1}{|c|}{ Area\% } \\
\hline 1 & 10.685 & 49.996 \\
\hline 2 & 13.602 & 50.004 \\
\hline Total & & 100.000 \\
\hline
\end{tabular}

$<$ Chromatogram>

mAU

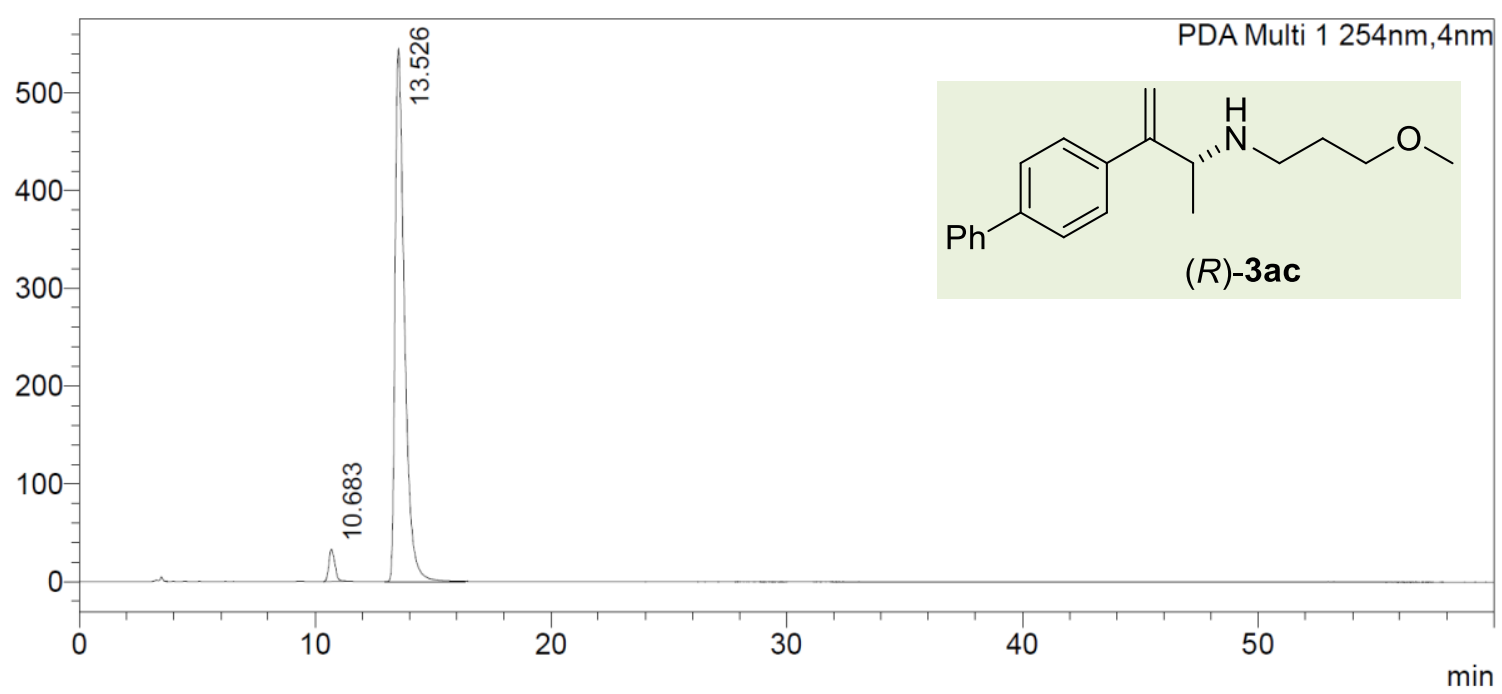

<Peak Table>

PDA Ch1 254nm

Peak\# Ret. Time $\quad$ Area\%

\begin{tabular}{|r|r|r|}
\hline 1 & 10.683 & 3.654 \\
\hline 2 & 13.526 & 96.346 \\
\hline Total & & 100.000 \\
\hline
\end{tabular}




\section{(R)- $N^{\top}$-(3-([1,1'-Biphenyl]-4-yl)but-3-en-2-yl)- $N^{\beta}, N^{\beta}$-dimethylpropane-1,3-diamine 3ad}

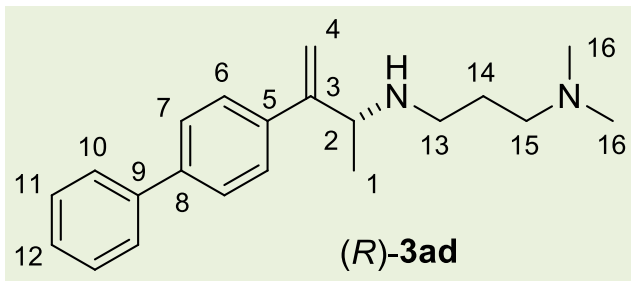

Synthesized at room temperature for $72 \mathrm{~h}$ following the general procedure using 4-(buta-1,3-dien-2-yl)1,1'-biphenyl 1a (51.6 mg, $0.25 \mathrm{mmol}, 1.0$ equiv), 3(dimethylamino)-1-propylamine $\mathbf{2 d}(126 \mu \mathrm{L}, 1.00$ mmol, 4.0 equiv), $\mathrm{Ni}(\operatorname{cod})_{2}(3.4 \mathrm{mg}, 0.0125 \mathrm{mmol}, 5$ mol\%), $(R, R)$-BenzP* (4.2 mg, $0.015 \mathrm{mmol}, 6 \mathrm{~mol} \%$ ), 2,2,2-trifluoroethanol (19 $\mu \mathrm{L}, 0.25$ mmol, 1.0 equiv) and mesitylene ( $0.50 \mathrm{~mL}, 0.5 \mathrm{M})$. Consumption of $1 \mathrm{a}$ : $>99 \%$, conversion of 3ad: $88 \%$, r.r. $=9: 1$. Purification by flash chromatography over silica gel $\left(\mathrm{CH}_{2} \mathrm{Cl}_{2}: \mathrm{MeOH}=\right.$ $3: 1$, with $1 \% \mathrm{Et}_{3} \mathrm{~N}$ ) led to the desired product as a yellow viscous oil $(66.2 \mathrm{mg}, 86 \%$ yield, $96 \%$ ee).

${ }^{1} \mathbf{H}$ NMR $\left(400 \mathrm{MHz}, \mathrm{CDCl}_{3}\right) \delta(\mathrm{ppm})=7.63-7.54(\mathrm{~m}, 4 \mathrm{H}, \mathrm{H}-7+\mathrm{H}-10), 7.50-7.41(\mathrm{~m}, 4 \mathrm{H}, \mathrm{H}-$ $6+H-11), 7.37-7.31(\mathrm{~m}, 1 \mathrm{H}, H-12), 5.32\left(\mathrm{~d},{ }^{2} \mathrm{~J}_{\mathrm{HH}}=1.1 \mathrm{~Hz}, 1 \mathrm{H}, H-4\right), 5.27(\mathrm{~s}, 1 \mathrm{H}, H-4) 3.73$ (q, $\left.{ }^{3} \mathrm{~J}_{\mathrm{HH}}=6.5 \mathrm{~Hz}, 1 \mathrm{H}, \mathrm{H}-2\right), 2.78-2.61(\mathrm{~m}, 2 \mathrm{H}, \mathrm{H}-13), 2.38-2.26(\mathrm{~m}, 2 \mathrm{H}, \mathrm{H}-15), 2.21(\mathrm{~s}, 6 \mathrm{H}$, $H-16), 1.68\left(\mathrm{p},{ }^{3} \mathrm{JHH}_{\mathrm{HH}}=7.1 \mathrm{~Hz}, 2 \mathrm{H}, H-14\right), 1.26(\mathrm{~s}, 1 \mathrm{H}, \mathrm{NH}), 1.24\left(\mathrm{~d},{ }^{3} J_{\mathrm{HH}}=6.6 \mathrm{~Hz}, 3 \mathrm{H}, \mathrm{H}-1\right)$.

${ }^{13} \mathrm{C}\left\{{ }^{1} \mathrm{H}\right\}$ NMR $\left(100 \mathrm{MHz}, \mathrm{CDCl}_{3}\right) \delta(\mathrm{ppm})=151.7(C-3), 140.9(C-9), 140.34(C-8), 140.30(C-$ 5), $128.9(\mathrm{CH}-11), 127.5(\mathrm{CH}-6), 127.4(\mathrm{CH}-12), 127.12(\mathrm{CH}-10), 127.05(\mathrm{CH}-7), 112.6\left(\mathrm{CH}_{2}-\right.$ 4), $58.3\left(\mathrm{CH}_{2}-15\right), 57.5(\mathrm{CH}-2), 46.1\left(\mathrm{CH}_{2}-13\right), 45.7\left(\mathrm{CH}_{3}-16\right), 28.4\left(\mathrm{CH}_{2}-14\right), 21.7\left(\mathrm{CH}_{3}-1\right)$.

HRMS $\left(\mathrm{ESI}^{+}\right)$: calculated $[\mathrm{M}+\mathrm{H}]^{+}$for $\mathrm{C}_{21} \mathrm{H}_{29} \mathrm{~N}_{2}{ }^{+}$: 309.2326; found: 296.2354.

IR (neat) $v\left(\mathrm{~cm}^{-1}\right): 3345,3029,2941,2764,1629,1589,1486,1462,1379,1265,1150,1081$, 1040, 1006, 909, 842, 769, 740, 697.

HPLC: $96 \%$ ee, chiral stationary column: AD-H, mobile phase: hexane/ $\mathrm{PrOH}=99 / 1,1.0$ $\mathrm{mL} / \mathrm{min}, 254 \mathrm{~nm}, 30^{\circ} \mathrm{C}, \mathrm{t}_{\mathrm{R}}$ (major) $=11.1 \mathrm{~min}, \mathrm{t}_{\mathrm{R}}$ (minor) $=14.7 \mathrm{~min}$. (After protecting the secondary amine with Boc (tert-butyloxycarbonyl) group, the enantiomeric excess of protected product can be determined by chiral AD-H column)

$[\alpha]^{20} \mathrm{D}=-2.7\left(c \quad 0.2, \mathrm{CHCl}_{3}\right)$. 
$<$ Chromatogram $>$

mAU

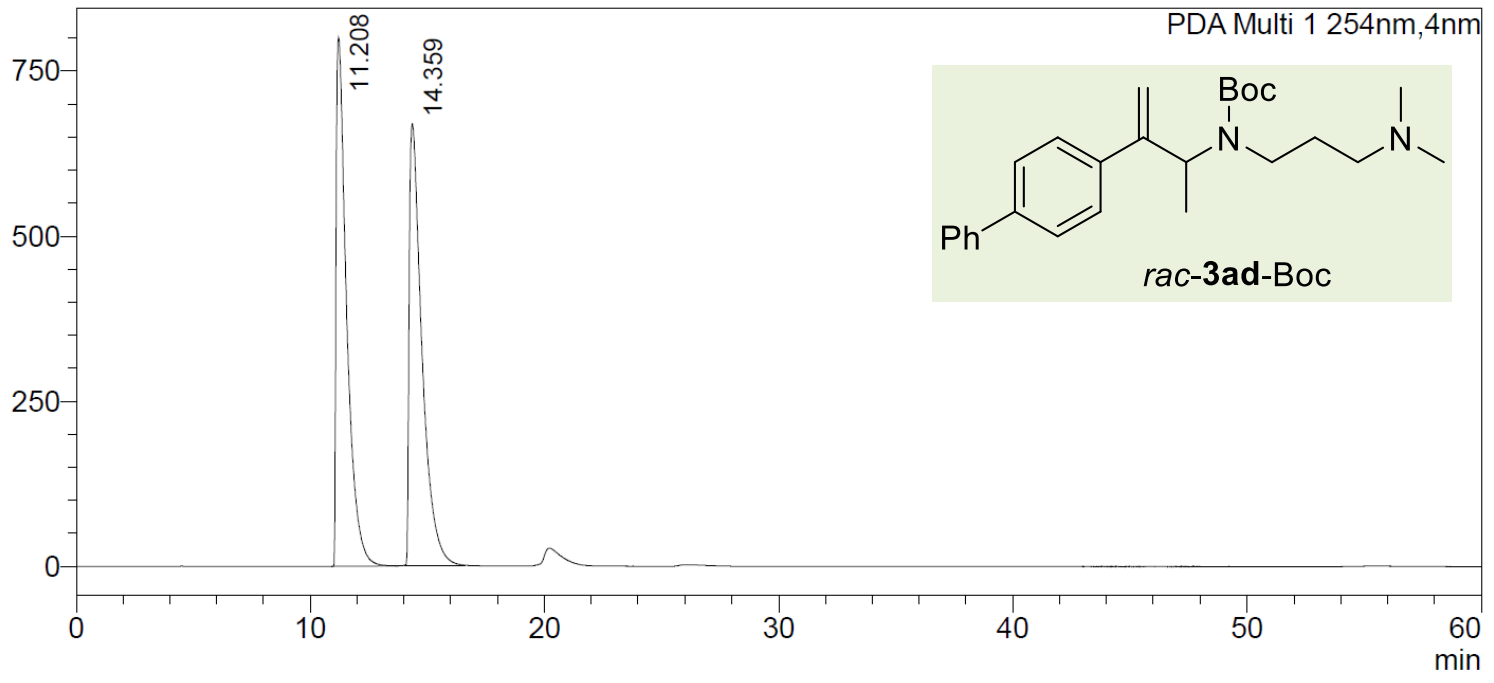

<Peak Table>

PDA Ch1 254nm

\begin{tabular}{|r|r|r|}
\hline Peak\# & Ret. Time & \multicolumn{1}{|c|}{ Area $\%$} \\
\hline 1 & 11.208 & 49.900 \\
\hline 2 & 14.359 & 50.100 \\
\hline Total & & 100.000 \\
\hline
\end{tabular}

$<$ Chromatogram $>$

mAU

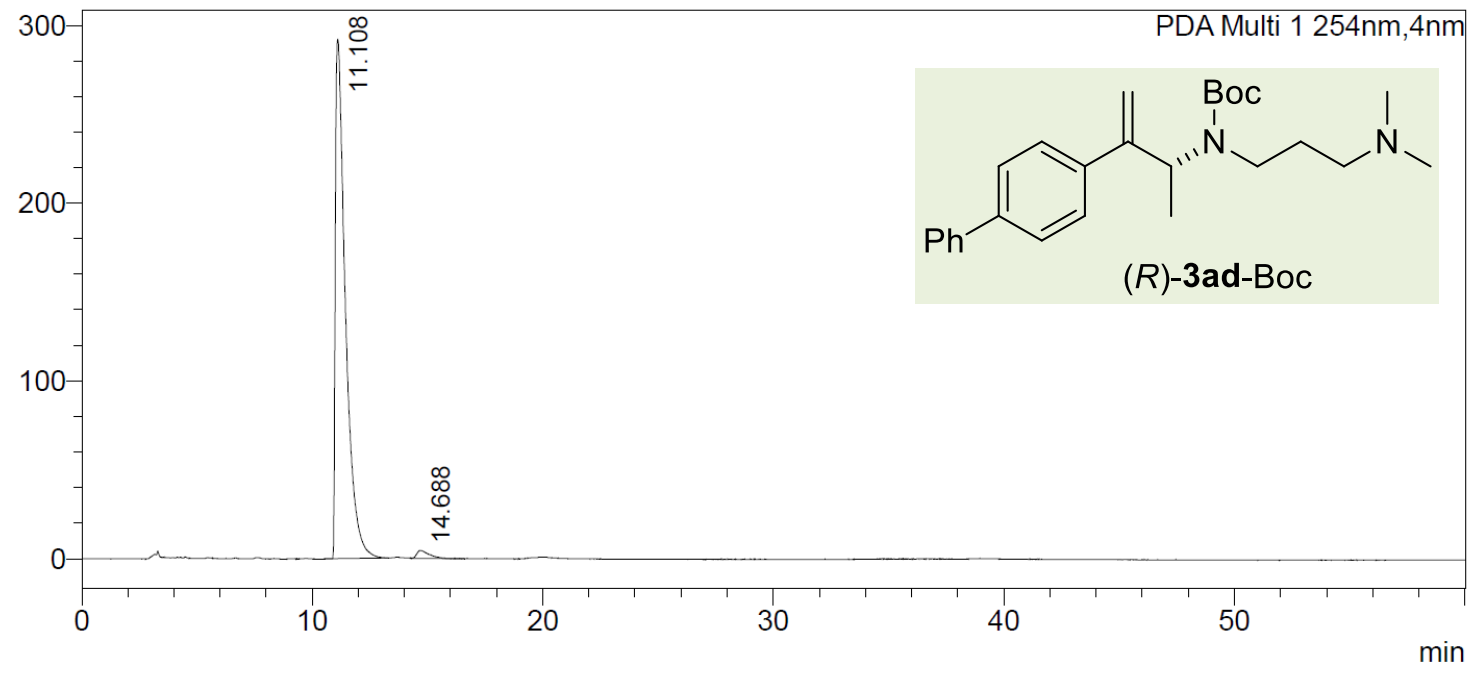

<Peak Table>

PDA Ch1 254nm

\begin{tabular}{|l|l}
\hline Peak\# Ret. Time & Area\% \\
\hline
\end{tabular}

\begin{tabular}{|r|r|r|}
\hline 1 & 11.108 & 98.241 \\
\hline 2 & 14.688 & 1.759 \\
\hline Total & & 100.000 \\
\hline
\end{tabular}




\section{(R)-3-([1,1'-Biphenyl]-4-yl)-N-(3,3,3-trifluoropropyl)but-3-en-2-amine 3ae}

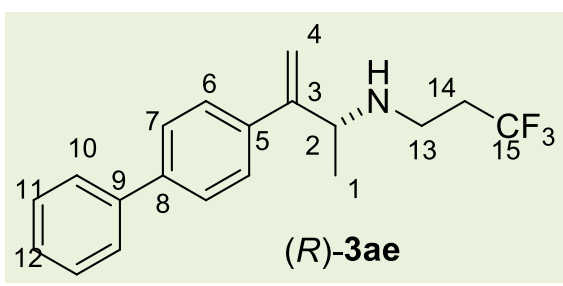

Synthesized at $40{ }^{\circ} \mathrm{C}$ for $72 \mathrm{~h}$ following the general procedure using 4-(buta-1,3-dien-2-yl)-1,1'-biphenyl 1a (52.0 mg, $0.25 \mathrm{mmol}, 1.0$ equiv), 3,3,3-trifluoropropan-1amine $2 \mathrm{e}\left(113.0 \mathrm{mg}, 1.00 \mathrm{mmol}, 4.0\right.$ equiv), $\mathrm{Ni}(\mathrm{cod})_{2}(3.4$ $\mathrm{mg}, 0.0125 \mathrm{mmol}, 5 \mathrm{~mol} \%),(R, R)-B^{2} \mathrm{BP}^{*}(4.2 \mathrm{mg}, 0.015$ mmol, 6 mol\%),2,2,2-trifluoroethanol ( $18 \mu \mathrm{L}, 0.25 \mathrm{mmol}, 1.0$ equiv) and mesitylene $(0.50 \mathrm{~mL}$, $0.5 \mathrm{M}$ ). Consumption of 1a: $>95 \%$, conversion of $3 \mathrm{ae}: 65 \%$, r.r. $=7: 1$. Purification by flash chromatography over silica gel (pentane:EtOAc $=6: 1$ ) led to the desired product as a pale yellow oil $(50.0 \mathrm{mg}, 63 \%$ yield, $75 \%$ ee).

${ }^{1} \mathrm{H}$ NMR $\left(500 \mathrm{MHz}, \mathrm{CDCl}_{3}\right) \delta(\mathrm{ppm})=7.64-7.58(\mathrm{~m}, 2 \mathrm{H}, \mathrm{H}-10), 7.61-7.54(\mathrm{~m}, 2 \mathrm{H}, \mathrm{H}-7)$, $7.50-7.41(\mathrm{~m}, 4 \mathrm{H}, \mathrm{H}-6+\mathrm{H}-11), 7.35\left(\mathrm{tt},{ }^{3} \mathrm{JHH}_{\mathrm{HH}}=6.9,{ }^{4} \mathrm{~J}_{\mathrm{HH}}=1.4 \mathrm{~Hz}, 1 \mathrm{H}, \mathrm{H}-12\right), 5.36(\mathrm{~s}, 1 \mathrm{H}$, $\mathrm{H}-4), 5.30(\mathrm{~s}, 1 \mathrm{H}, \mathrm{H}-4), 3.75\left(\mathrm{q},{ }^{3} \mathrm{~J}_{\mathrm{HH}}=6.5 \mathrm{~Hz}, 1 \mathrm{H}, \mathrm{H}-2\right), 2.97\left(\mathrm{dt},{ }^{2} \mathrm{~J}_{\mathrm{HH}}=12.2,{ }^{3} J_{\mathrm{HH}}=7.4 \mathrm{~Hz}\right.$, $1 \mathrm{H}, \mathrm{H}-13), 2.88\left(\mathrm{dt},{ }^{2} J_{\mathrm{HH}}=12.2,{ }^{3} J_{\mathrm{HH}}=6.9 \mathrm{~Hz}, 1 \mathrm{H}, \mathrm{H}-13\right), 2.34\left(\mathrm{dtd}_{\mathrm{app}}, J=14.3,10.7,5.4 \mathrm{~Hz}\right.$, $2 \mathrm{H}, \mathrm{H}-14), 1.56(\mathrm{~s}, 1 \mathrm{H}, \mathrm{N}-H), 1.27\left(\mathrm{~d},{ }^{3} \mathrm{~J}_{\mathrm{HH}}=6.5 \mathrm{~Hz}, 3 \mathrm{H}, \mathrm{H}-1\right)$.

${ }^{13} \mathrm{C}\left\{{ }^{1} \mathrm{H}\right\}$ NMR $\left(130 \mathrm{MHz}, \mathrm{CDCl}_{3}\right) \delta(\mathrm{ppm})=151.0(C-3), 140.8(C-9), 140.5(C-8), 139.9(C-5)$, $128.9(\mathrm{CH}-11), 127.48(\mathrm{CH}-12), 127.44(\mathrm{CH}-6), 127.1(\mathrm{CH}-7+\mathrm{CH}-10), 126.8\left(\mathrm{q},{ }^{1} \mathrm{~J}_{\mathrm{CF}}=277\right.$ $\mathrm{Hz}, \mathrm{C}-15), 113.1\left(\mathrm{CH}_{2}-4\right), 57.5(\mathrm{CH}-2), 40.3\left(\mathrm{q},{ }^{3} \mathrm{~J}_{\mathrm{CF}}=2 \mathrm{~Hz}, \mathrm{CH}_{2}-13\right), 34.7\left(\mathrm{q},{ }^{2} \mathrm{~J}_{\mathrm{CF}}=27 \mathrm{~Hz}\right.$, $\left.\mathrm{CH}_{2}-14\right), 21.7\left(\mathrm{CH}_{3}-1\right)$.

${ }^{19} \mathrm{~F}\left\{{ }^{1} \mathrm{H}\right\}$ NMR $\left(280 \mathrm{MHz}\right.$, Toluene- $\left.d_{8}\right) \delta(\mathrm{ppm})=-65.00$.

HRMS $\left(E S I^{+}\right)$: calculated $[\mathrm{M}+\mathrm{H}]^{+}$for $\mathrm{C}_{19} \mathrm{H}_{20} \mathrm{NF}_{3}{ }^{+}$: 320.1621 ; found: 320.1646 .

IR (neat) $v\left(\mathrm{~cm}^{-1}\right): 3030,2967,1625,1601,1487,1444,1373,1340,1252,1113,1004,911$, 844, 770, 740, 696.

HPLC: $75 \%$ ee, chiral stationary column: $\mathrm{OJ}-\mathrm{H}$, mobile phase: hexane/iPrOH $=99 / 1,1.0$ $\mathrm{mL} / \mathrm{min}, 254 \mathrm{~nm}, 30^{\circ} \mathrm{C}, \mathrm{t}_{\mathrm{R}}$ (minor) $=29.5 \mathrm{~min}, \mathrm{t}_{\mathrm{R}}$ (major) $=39.1 \mathrm{~min}$.

$[\alpha]^{20} \mathrm{D}=-33.96\left(c \quad 0.25, \mathrm{CH}_{2} \mathrm{Cl}_{2}\right)$. 
$<$ Chromatogram $>$

mAU

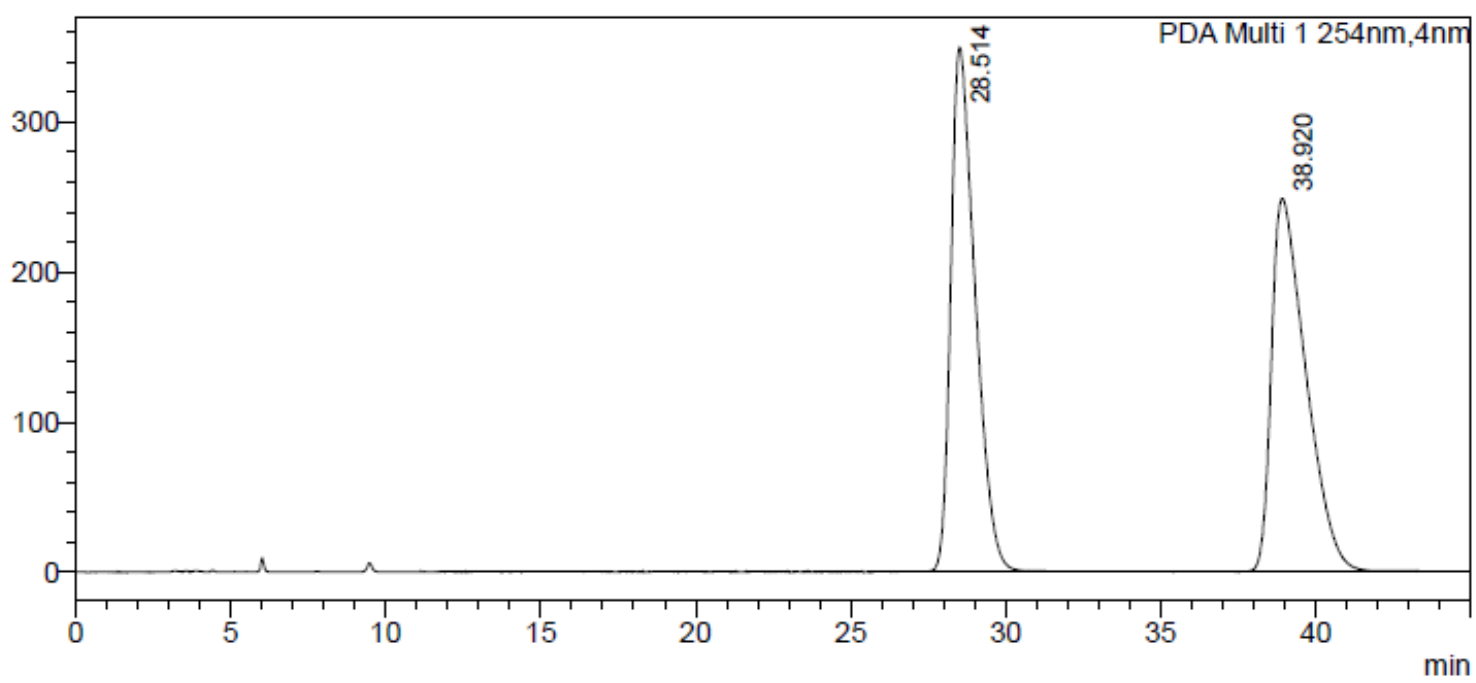

<Peak Table>

PDA Ch1 254nm

\begin{tabular}{|l|l|}
\hline Peak\# & Ret. Time \\
\hline
\end{tabular}

\begin{tabular}{|r|r|r|}
\hline 1 & 28.514 & 49.884 \\
\hline 2 & 38.920 & 50.116 \\
\hline Total & & 100.000 \\
\hline
\end{tabular}

$<$ Chromatogram>

mAU

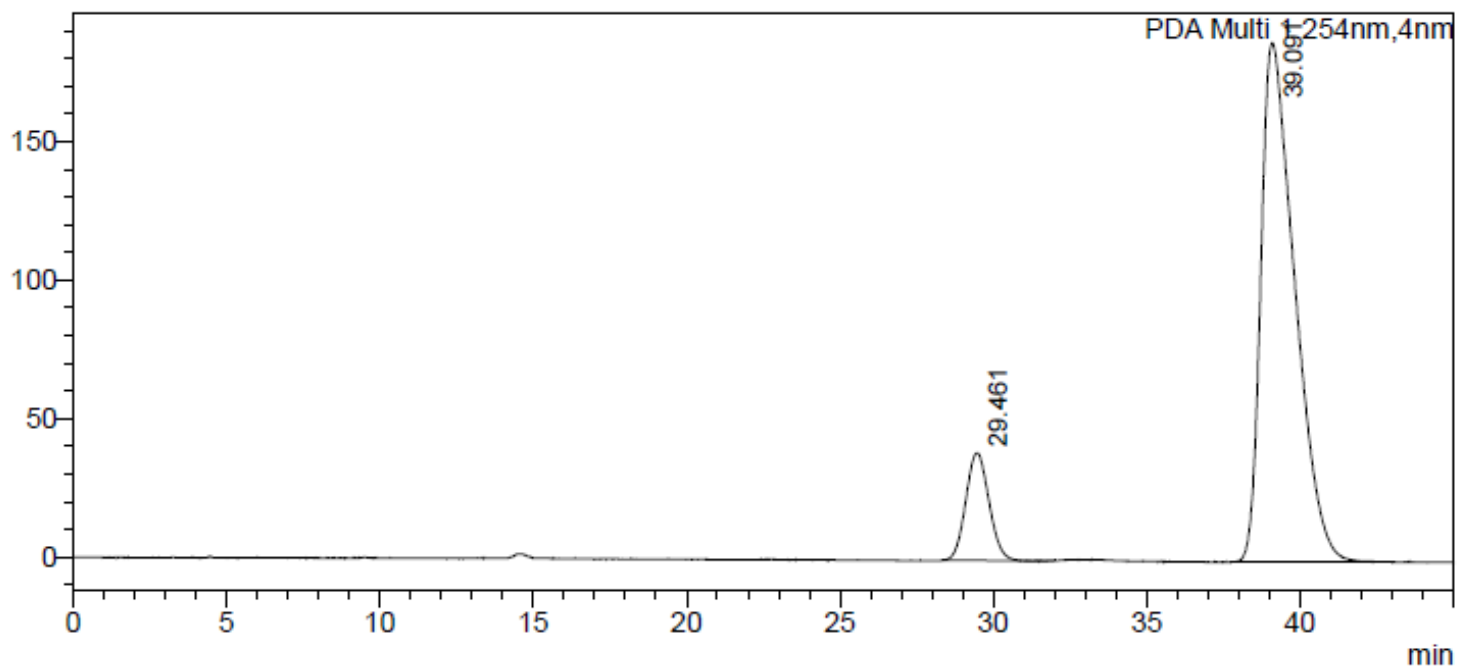

<Peak Table>

PDA Ch1 254nm

\begin{tabular}{|l|l|}
\hline Peak\# Ret. Time & Area\% \\
\hline
\end{tabular}

\begin{tabular}{|r|r|r|}
\hline 1 & 29.461 & 12.403 \\
\hline 2 & 39.091 & 87.597 \\
\hline Total & & 100.000 \\
\hline
\end{tabular}




\section{(R)-3-([1,1'-Biphenyl]-4-yl)-N-(pyridin-3-ylmethyl)but-3-en-2-amine 3af}

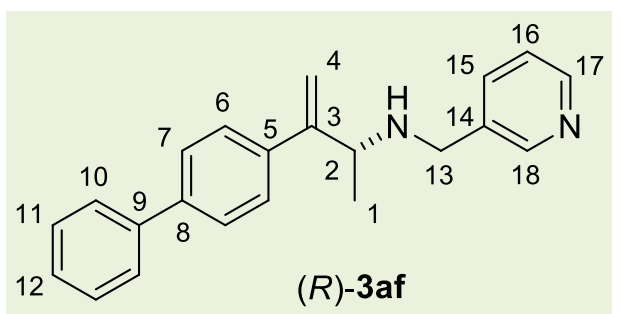

Synthesized at room temperature for $72 \mathrm{~h}$ following the general procedure using 4-(buta-1,3-dien-2-yl)-1,1'biphenyl 1a (51.6 mg, 0.25 mmol, 1.0 equiv), 3picolylamine $2 f(114 \mu \mathrm{L}, 1.00 \mathrm{mmol}, 4.0$ equiv), $\mathrm{Ni}(\operatorname{cod})_{2}$ (3.4 mg, $\left.0.0125 \mathrm{mmol}, 5 \mathrm{~mol} \%\right),(R, R)$-BenzP* (4.2 mg, $0.015 \mathrm{mmol}, 6 \mathrm{~mol} \%$ ), 2,2,2-trifluoroethanol (19 $\mu \mathrm{L}, 0.25 \mathrm{mmol}, 1.0$ equiv) and mesitylene $(0.50 \mathrm{~mL}, 0.5 \mathrm{M})$. Consumption of 1a: $94 \%$, conversion of 3af: $68 \%$, r.r. $=7: 1$. Purification by flash chromatography over silica gel $\left(\mathrm{CH}_{2} \mathrm{Cl}_{2}: \mathrm{MeOH}=20: 1\right)$ led to the desired product as a white solid $(64.0 \mathrm{mg}, 81 \%$ yield, $93 \%$ ee) .

${ }^{1} \mathrm{H}$ NMR $\left(400 \mathrm{MHz}, \mathrm{CDCl}_{3}\right) \delta(\mathrm{ppm})=8.58\left(\mathrm{~d},{ }^{4} \mathrm{~J}_{\mathrm{HH}}=1.7 \mathrm{~Hz}, 1 \mathrm{H}, \mathrm{H}-18\right), 8.51\left(\mathrm{dd},{ }^{3} \mathrm{~J}_{\mathrm{HH}}=4.8\right.$, $\left.{ }^{4} J_{\mathrm{HH}}=1.6 \mathrm{~Hz}, 1 \mathrm{H}, H-17\right), 7.67\left(\mathrm{dt},{ }^{3} J_{\mathrm{HH}}=7.8,{ }^{4} J_{\mathrm{HH}}=1.9 \mathrm{~Hz}, 1 \mathrm{H}, H-15\right), 7.64-7.53(\mathrm{~m}, 4 \mathrm{H}, \mathrm{H}-$ $7+H-10), 7.51-7.41(\mathrm{~m}, 4 \mathrm{H}, H-6+H-11), 7.39-7.31(\mathrm{~m}, 1 \mathrm{H}, H-12), 7.25\left(\mathrm{dd}, 1 \mathrm{H},{ }^{3} J_{H H}=7.6\right.$, $4.8 \mathrm{~Hz}, 1 \mathrm{H}, H-16), 5.39\left(\mathrm{~d},{ }^{2} J_{\mathrm{HH}}=1.3 \mathrm{~Hz}, 1 \mathrm{H}, H-4\right), 5.35(\mathrm{~s}, 1 \mathrm{H}, H-4), 3.90\left(\mathrm{~d},{ }^{2} J_{\mathrm{HH}}=13.4 \mathrm{~Hz}\right.$, $1 \mathrm{H}, H-13), 3.83-3.73(\mathrm{~m}, 2 \mathrm{H}, H-2+H-13), 1.50(\mathrm{~s}, 1 \mathrm{H}, \mathrm{NH}), 1.29\left(\mathrm{~d},{ }^{3} \mathrm{JHH}_{\mathrm{H}}=6.6 \mathrm{~Hz}, 3 \mathrm{H}, H-1\right)$. ${ }^{13} \mathrm{C}\left\{{ }^{1} \mathrm{H}\right\}$ NMR $\left(100 \mathrm{MHz}, \mathrm{CDCl}_{3}\right) \delta(\mathrm{ppm})=151.3(\mathrm{C}-3), 149.9(\mathrm{CH}-18), 148.6(\mathrm{CH}-17), 140.8$ (C-9), 140.5 (C-8), 140.0 (C-5), 136.1 (C-14), 136.0 ( $\mathrm{CH}-15), 128.9(\mathrm{CH}-11), 127.49(\mathrm{CH}-6)$, $127.45(\mathrm{CH}-12), 127.13(\mathrm{CH}-10), 127.11(\mathrm{CH}-7), 123.5(\mathrm{CH}-16), 113.1\left(\mathrm{CH}_{2}-4\right), 57.0(\mathrm{CH}-2)$, $48.8\left(\mathrm{CH}_{2}-13\right), 21.8\left(\mathrm{CH}_{3}-1\right)$.

m.p. $=84.1-86.4^{\circ} \mathrm{C}$.

HRMS $\left(E S I^{+}\right)$: calculated $[\mathrm{M}+\mathrm{H}]^{+}$for $\mathrm{C}_{22} \mathrm{H}_{23} \mathrm{~N}_{2}{ }^{+}: 315.1856$; found: 315.1881 .

IR (neat) $v\left(\mathrm{~cm}^{-1}\right): 3291,3034,2964,2927,2825,1621,1579,1486,1446,1423,1374,1158$, 1128, 1085, 1005, 903, 842, 770, 740, 711, 690.

HPLC: $93 \%$ ee, chiral stationary column: $\mathrm{OJ}-\mathrm{H}$, mobile phase: hexane/ $\mathrm{PrOH}=85 / 15,1.0$ $\mathrm{mL} / \mathrm{min}, 254 \mathrm{~nm}, 30^{\circ} \mathrm{C}, \mathrm{t}_{\mathrm{R}}($ minor $)=25.6 \mathrm{~min}, \mathrm{t}_{\mathrm{R}}$ (major) $=27.8 \mathrm{~min}$.

$[\alpha]^{20}=+10.7\left(c 1.0, \mathrm{CHCl}_{3}\right)$. 
<Chromatogram>

mAU

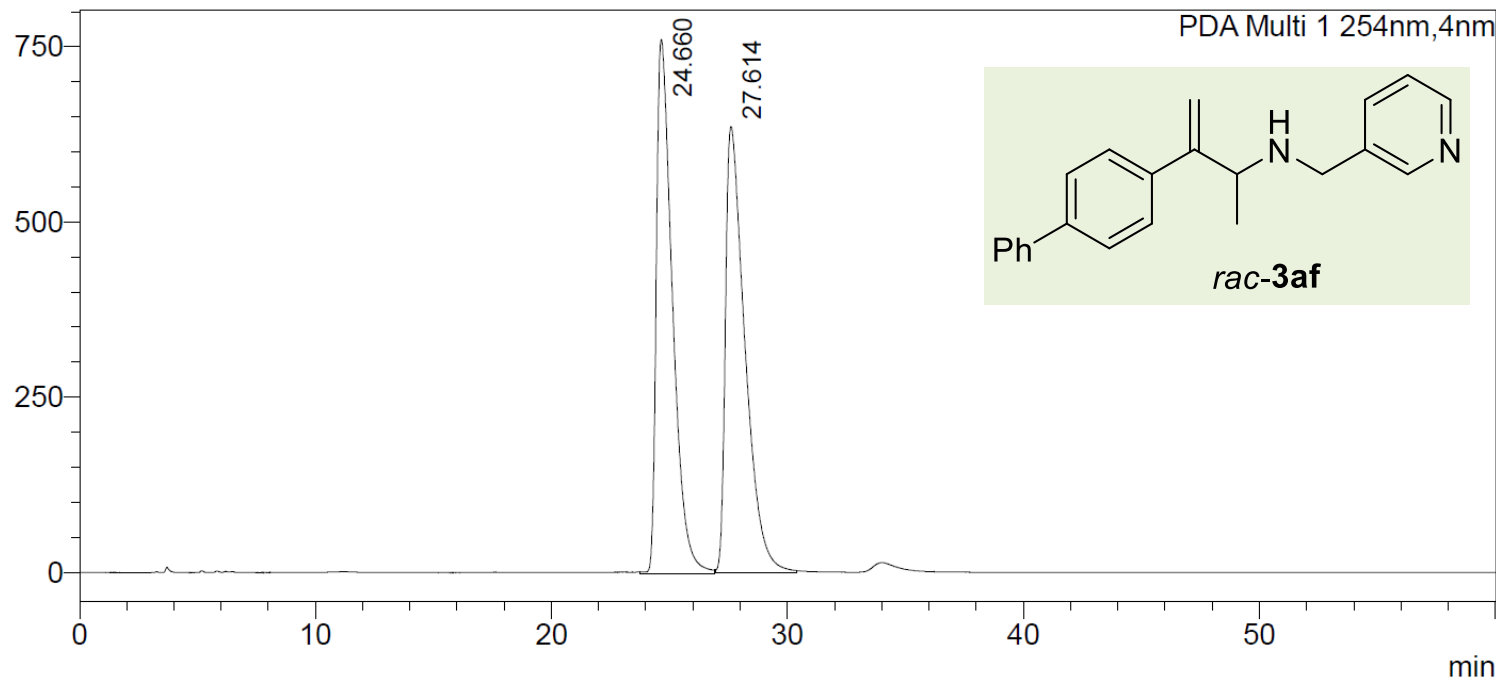

<Peak Table>

PDA Ch1 254nm

\begin{tabular}{|r|r|r|}
\hline Peak\# & Ret. Time & \multicolumn{1}{|c|}{ Area $\%$} \\
\hline 1 & 24.660 & 49.958 \\
\hline 2 & 27.614 & 50.042 \\
\hline Total & & 100.000 \\
\hline
\end{tabular}

<Chromatogram>

mAU

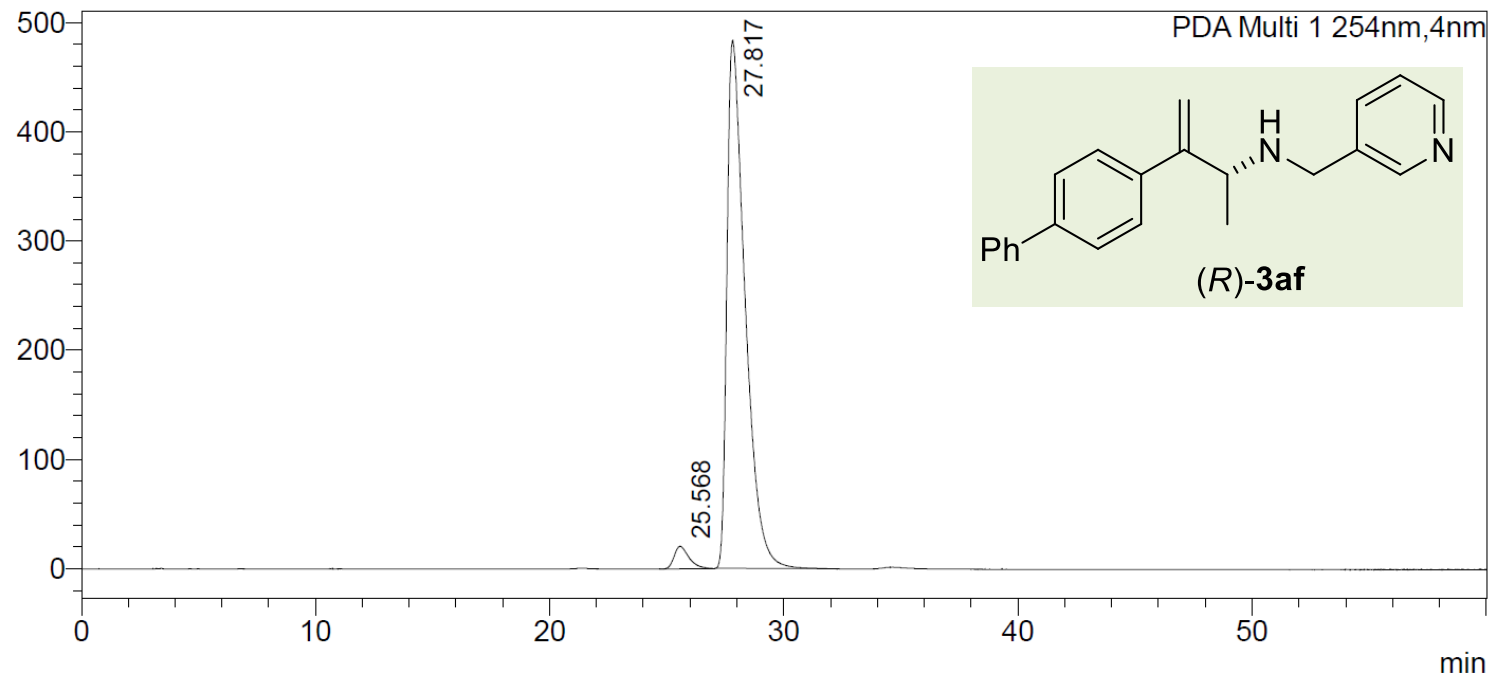

<Peak Table>

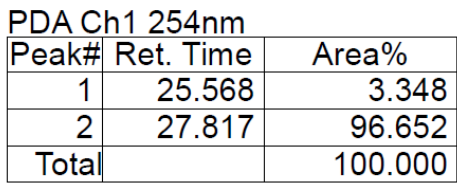




\section{(R)-3-([1,1'-Biphenyl]-4-yl)-N-(furan-2-ylmethyl)but-3-en-2-amine 3ag}

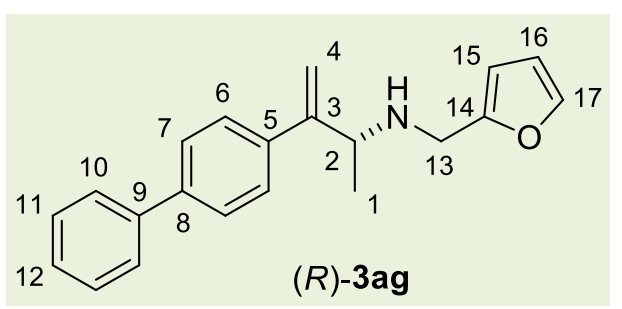

Synthesized at room temperature for $72 \mathrm{~h}$ following the general procedure using 4-(buta-1,3-dien-2-yl)-1,1'biphenyl 1a (51.6 mg, 0.25 mmol, 1.0 equiv), furfurylamine $\mathbf{2 g}$ (88 $\mu \mathrm{L}, 1.00 \mathrm{mmol}, 4.0$ equiv), $\mathrm{Ni}(\operatorname{cod})_{2}$ (3.4 mg, $\left.0.0125 \mathrm{mmol}, 5 \mathrm{~mol} \%\right),(R, R)-B e n z \mathrm{P}^{*}$ (4.2 mg, $0.015 \mathrm{mmol}, 6 \mathrm{~mol} \%)$, 2,2,2-trifluoroethanol (19 $\mu \mathrm{L}, 0.25 \mathrm{mmol}, 1.0$ equiv) and mesitylene $(0.50 \mathrm{~mL}, 0.5 \mathrm{M})$. Consumption of $1 \mathrm{a}: 97 \%$, conversion of $3 \mathrm{ag}: 80 \%$, r.r. $=6: 1$. Purification by flash chromatography over silica gel (Pentane:EtOAc $=4: 1$ ) led to the desired product as a colorless viscous oil $(56.7 \mathrm{mg}, 75 \%$ yield, $91 \%$ ee).

${ }^{1} \mathbf{H}$ NMR $\left(400 \mathrm{MHz}, \mathrm{CDCl}_{3}\right) \delta(\mathrm{ppm})=7.65-7.54(\mathrm{~m}, 4 \mathrm{H}, \mathrm{H}-7+\mathrm{H}-10), 7.50-7.41(\mathrm{~m}, 4 \mathrm{H}, \mathrm{H}-$ $6+H-11), 7.41-7.38(\mathrm{~m}, 1 \mathrm{H}, H-17), 7.38-7.31(\mathrm{~m}, 1 \mathrm{H} \mathrm{H}-12), 6.37-6.31(\mathrm{~m}, 1 \mathrm{H}, \mathrm{H}-16)$, $6.19\left(\mathrm{~d},{ }^{3} J_{\mathrm{HH}}=3.1 \mathrm{~Hz}, 1 \mathrm{H}, H-15\right), 5.38(\mathrm{~s}, 1 \mathrm{H}, \mathrm{H}-4), 5.35(\mathrm{~s}, 1 \mathrm{H}, \mathrm{H}-4), 3.89\left(\mathrm{~d},{ }^{2} J_{\mathrm{HH}}=14.4 \mathrm{~Hz}\right.$, $1 \mathrm{H}, H-13), 3.85-3.73(\mathrm{~m}, 2 \mathrm{H}, H-2+H-13), 1.65(\mathrm{~s}, 1 \mathrm{H}, \mathrm{NH}), 1.25\left(\mathrm{~d},{ }^{3} \mathrm{JHH}_{\mathrm{H}}=6.5 \mathrm{~Hz}, 3 \mathrm{H}, H-1\right)$. ${ }^{13} \mathrm{C}\left\{{ }^{1} \mathrm{H}\right\}$ NMR $\left(100 \mathrm{MHz}, \mathrm{CDCl}_{3}\right) \delta(\mathrm{ppm})=154.3(\mathrm{C}-14), 151.2(\mathrm{C}-3), 141.9(\mathrm{CH}-17), 140.9$ (C-9), $140.4(C-5), 140.2(C-8), 128.9(\mathrm{CH}-11), 127.5(\mathrm{CH}-6), 127.4(\mathrm{CH}-12), 127.14(\mathrm{CH}-$ 10), $127.07(\mathrm{CH}-7), 113.0\left(\mathrm{CH}_{2}-4\right), 110.3(\mathrm{CH}-16), 107.0(\mathrm{CH}-15), 56.2(\mathrm{CH}-2), 43.8\left(\mathrm{CH}_{2}-\right.$ 13), $21.7\left(\mathrm{CH}_{3}-1\right)$.

HRMS $\left(\mathrm{ESI}^{+}\right)$: calculated $[\mathrm{M}+\mathrm{H}]^{+}$for $\mathrm{C}_{21} \mathrm{H}_{22} \mathrm{NO}^{+}:$304.1696; found: 304.1701.

IR (neat) $v\left(\mathrm{~cm}^{-1}\right):$ 3030, 2965, 2926, 2852, 1810, 1624, 1600, 1505, 1486, 1447, 1401, 1370, 1148, 1074, 1008, 912, 844, 770, 735, 695, 598.

HPLC: $91 \%$ ee, chiral stationary column: $\mathrm{OJ}-\mathrm{H}$, mobile phase: hexane/ $\mathrm{PrOH}=90 / 10,0.50$ $\mathrm{mL} / \mathrm{min}, 254 \mathrm{~nm}, 30^{\circ} \mathrm{C}, \mathrm{t}_{\mathrm{R}}($ minor $)=29.1 \mathrm{~min}, \mathrm{t}_{\mathrm{R}}$ (major) $=30.8 \mathrm{~min}$.

$[\alpha]^{20}=+11.8\left(c 1.0, \mathrm{CHCl}_{3}\right)$. 
<Chromatogram>

mAU

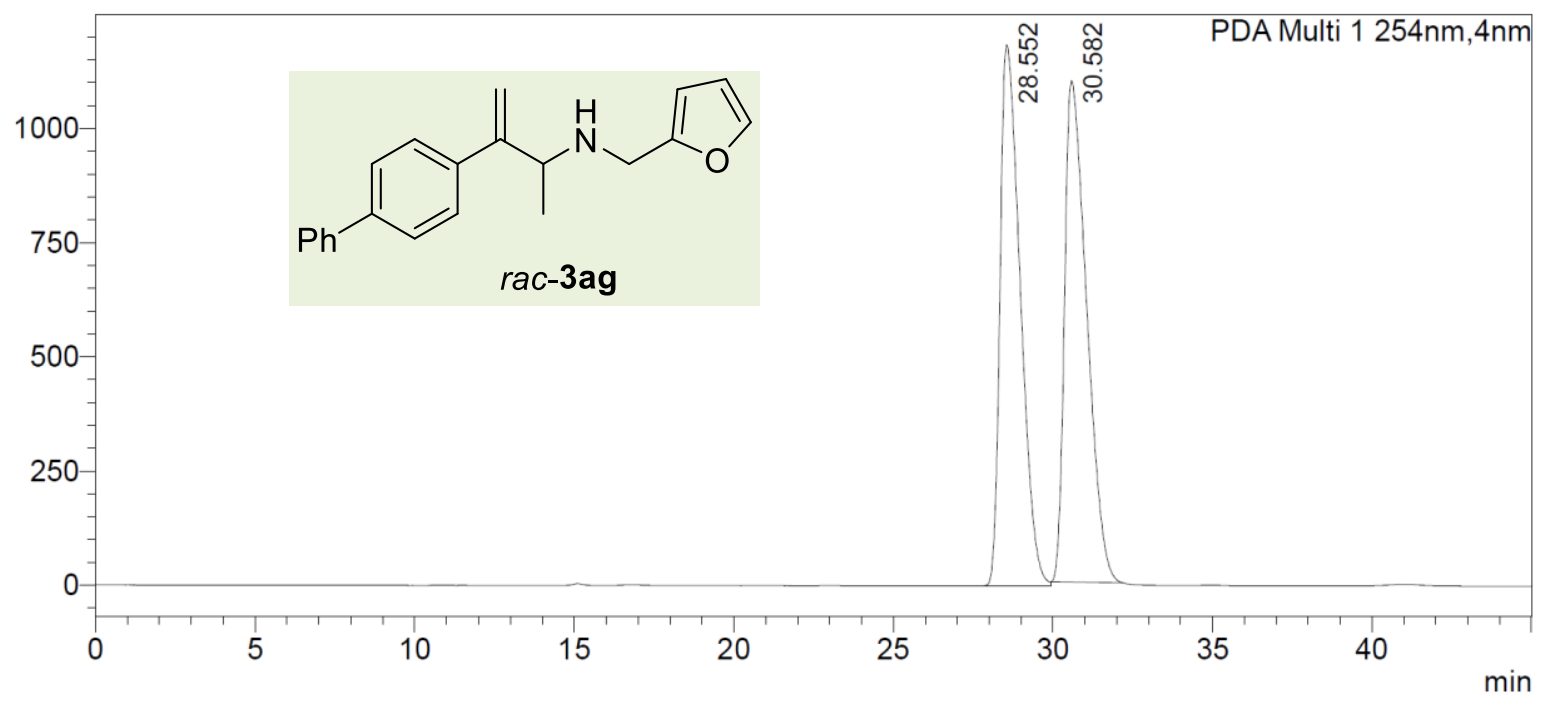

<Peak Table>

PDA Ch1 254nm

\begin{tabular}{l|l}
\hline Peak\# Ret. Time & Area\%
\end{tabular}

\begin{tabular}{|r|r|r|}
\hline 1 & 28.552 & 49.789 \\
\hline 2 & 30.582 & 50.211 \\
\hline Total & & 100.000 \\
\hline
\end{tabular}

\section{<Chromatogram>}

mAU

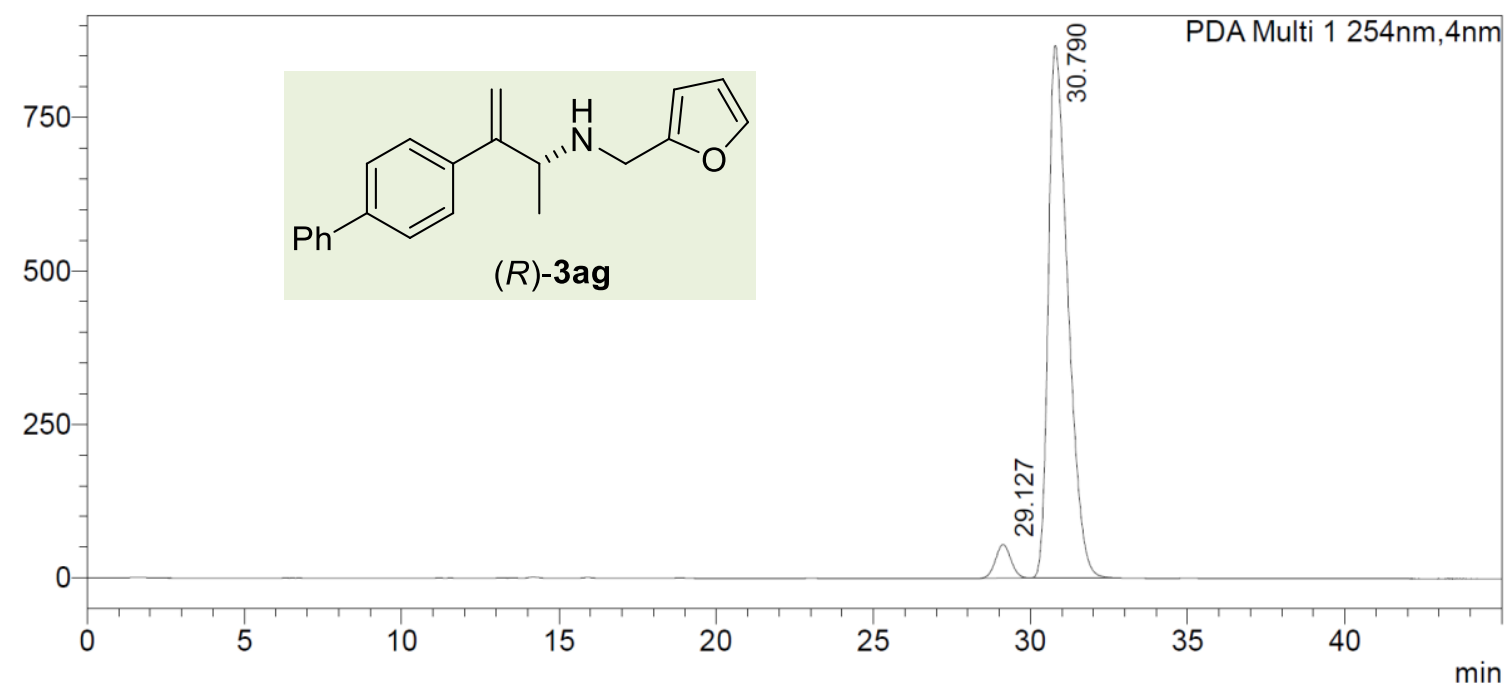

<Peak Table>

PDA Ch1 254nm

\begin{tabular}{|l|l}
\hline Peak\# Ret. Time & Area\% \\
\hline
\end{tabular}

\begin{tabular}{|r|r|r|}
\hline 1 & 29.127 & 4.692 \\
\hline 2 & 30.790 & 95.308 \\
\hline Total & & 100.000 \\
\hline
\end{tabular}




\section{(R)-4-(2-((3-([1,1'-Biphenyl]-4-yl)but-3-en-2-yl)amino)ethyl)aniline 3ah}

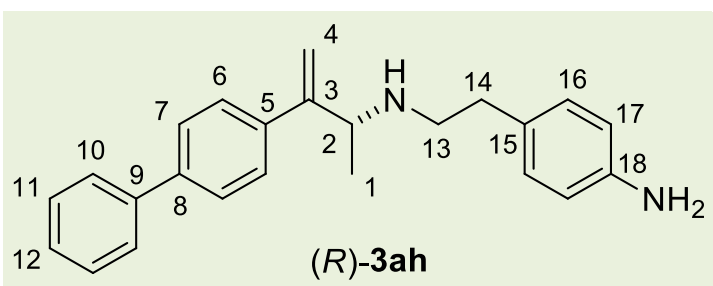

Synthesized at room temperature for $96 \mathrm{~h}$ following the general procedure using 4-(buta1,3-dien-2-yl)-1,1'-biphenyl 1a (51.6 mg, 0.25 mmol, 1.0 equiv), 4-(2-aminoethyl)aniline $2 \mathrm{~h}$ (136 mg, $1.00 \mathrm{mmol}, 4.0$ equiv), $\mathrm{Ni}(\operatorname{cod})_{2}(3.4$ $\mathrm{mg}, 0.0125 \mathrm{mmol}, 5 \mathrm{~mol} \%),(R, R)$-BenzP* $(4.2 \mathrm{mg}, 0.015 \mathrm{mmol}, 6 \mathrm{~mol} \%), 2,2,2-$ trifluoroethanol (19 $\mu \mathrm{L}, 0.25 \mathrm{mmol}, 1.0$ equiv) and mesitylene $(0.50 \mathrm{~mL}, 0.5 \mathrm{M})$. Consumption of 1a: $99 \%$, conversion of 3 ah: $74 \%$, r.r. $=8: 1$. Purification by flash chromatography over silica gel (Pentane:EtOAc $=1: 2$, with $0.2 \% \mathrm{Et}_{3} \mathrm{~N}$ ) led to the desired product as a yellow solid (60.6 mg, $71 \%$ yield, $87 \%$ ee).

${ }^{1} \mathrm{H}$ NMR $\left(400 \mathrm{MHz}, \mathrm{CDCl}_{3}\right) \delta(\mathrm{ppm})=7.64-7.59(\mathrm{~m}, 2 \mathrm{H}, H-10), 7.56-7.51(\mathrm{~m}, 2 \mathrm{H}, \mathrm{H}-7)$, $7.48-7.42(\mathrm{~m}, 2 \mathrm{H}, \mathrm{H}-11), 7.42-7.38(\mathrm{~m}, 2 \mathrm{H}, \mathrm{H}-6), 7.38-7.33(\mathrm{~m}, 1 \mathrm{H}, \mathrm{H}-12), 6.99\left(\mathrm{~d},{ }^{3} \mathrm{~J}_{\mathrm{HH}}\right.$ $=8.3 \mathrm{~Hz}, 2 \mathrm{H}, H-16), 6.68-6.54(\mathrm{~m}, 2 \mathrm{H}, H-17), 5.29\left(\mathrm{~d},{ }^{2} \mathrm{JHH}_{\mathrm{H}}=1.3 \mathrm{~Hz}, 1 \mathrm{H}, H-4\right), 5.20(\mathrm{~s}, 1 \mathrm{H}$, $\mathrm{H}-4), 3.72\left(\mathrm{q},{ }^{3} \mathrm{JHH}_{\mathrm{HH}}=6.5 \mathrm{~Hz}, 1 \mathrm{H}, H-2\right), 3.53\left(\mathrm{~s}, 2 \mathrm{H}, \mathrm{NH}_{2}\right), 2.94-2.81(\mathrm{~m}, 2 \mathrm{H}, \mathrm{H}-13), 2.79-$ $2.64(\mathrm{~m}, 2 \mathrm{H}, H-14), 1.37(\mathrm{~s}, 1 \mathrm{H}, \mathrm{NH}), 1.22\left(\mathrm{~d},{ }^{3} \mathrm{~J}_{\mathrm{HH}}=6.6 \mathrm{~Hz}, 3 \mathrm{H}, H-1\right)$.

${ }^{13} \mathrm{C}\left\{{ }^{1} \mathrm{H}\right\}$ NMR $\left(100 \mathrm{MHz}, \mathrm{CDCl}_{3}\right) \delta(\mathrm{ppm})=151.8(C-3), 144.7(C-18), 140.9(C-9), 140.3(C-$ 5), $140.2(\mathrm{C}-8), 130.2(\mathrm{C}-15), 129.6(\mathrm{CH}-16), 128.9(\mathrm{CH}-11), 127.5(\mathrm{CH}-6), 127.4(\mathrm{CH}-12)$, $127.1(\mathrm{CH}-10), 127.0(\mathrm{CH}-7), 115.4(\mathrm{CH}-17), 112.6\left(\mathrm{CH}_{2}-4\right), 57.4(\mathrm{CH}-2), 49.0\left(\mathrm{CH}_{2}-13\right), 35.7$ $\left(\mathrm{CH}_{2}-14\right), 21.6\left(\mathrm{CH}_{3}-1\right)$.

m.p. $=61.9-64.0^{\circ} \mathrm{C}$.

HRMS (ESI+): calculated $[\mathrm{M}+\mathrm{H}]^{+}$for $\mathrm{C}_{24} \mathrm{H}_{27} \mathrm{~N}_{2}{ }^{+}: 343.2169$; found: 343.2211 .

IR (neat) $v\left(\mathrm{~cm}^{-1}\right): 3452,3358,3204,3028,2966,2924,2845,1620,1515,1486,1445,1369$, 1272, 1179, 1149, 1122, 1086, 1007, 907, 843, 819, 770, 739, 695.

HPLC: $93 \%$ ee, chiral stationary column: AD-H, mobile phase: hexane/ $\mathrm{PrOH}=97 / 3,1.0$ $\mathrm{mL} / \mathrm{min}, 254 \mathrm{~nm}, 30^{\circ} \mathrm{C}, \mathrm{t}_{\mathrm{R}}($ minor $)=37.0 \mathrm{~min}, \mathrm{t}_{\mathrm{R}}$ (major) $=39.7 \mathrm{~min}$.

$[\alpha]^{20} \mathrm{D}=+0.2\left(c 1.0, \mathrm{CHCl}_{3}\right)$. 
<Chromatogram>

mAU

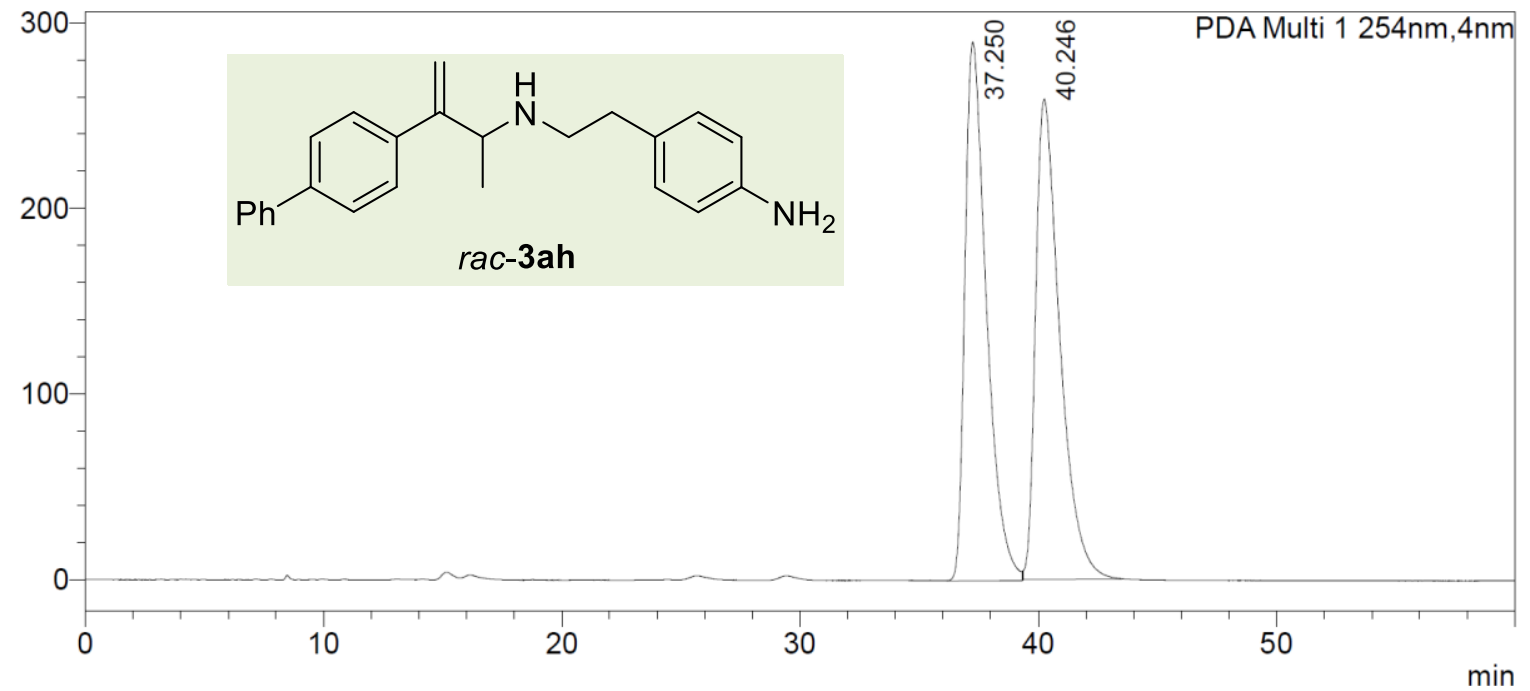

<Peak Table>

PDA Ch1 254nm

Peak\# Ret. Time $\quad$ Area\%

\begin{tabular}{|r|r|r|}
\hline 1 & 37.250 & 49.936 \\
\hline 2 & 40.246 & 50.064 \\
\hline Total & & 100.000 \\
\hline
\end{tabular}

<Chromatogram>

mAU

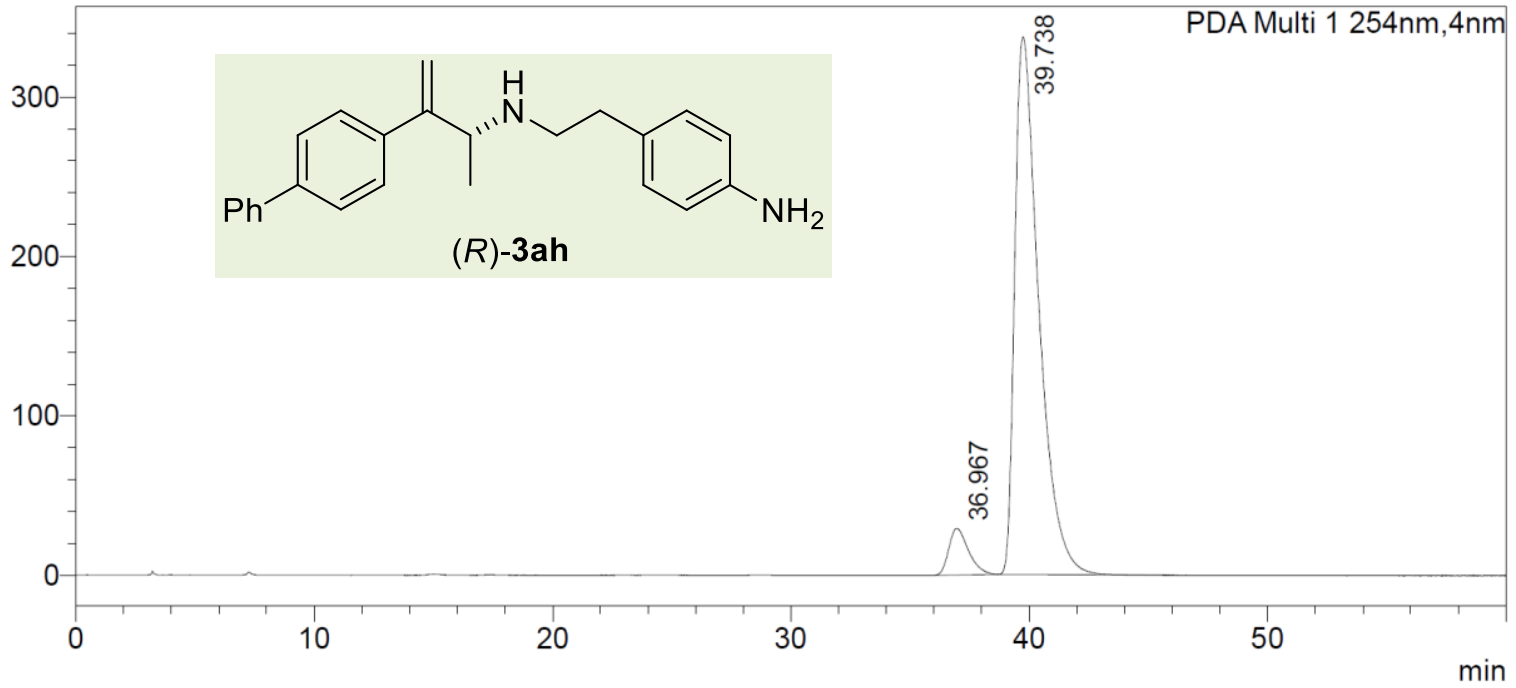

<Peak Table>

PDA Ch1 254nm

\begin{tabular}{|l|l}
\hline Peak\# Ret. Time & Area\% \\
\hline
\end{tabular}

\begin{tabular}{|r|r|r|}
\hline 1 & 36.967 & 6.646 \\
\hline 2 & 39.738 & 93.354 \\
\hline Total & & 100.000 \\
\hline
\end{tabular}




\section{(R)-N-(3-([1,1'-Biphenyl]-4-yl)but-3-en-2-yl)cyclopentanamine 3ai}

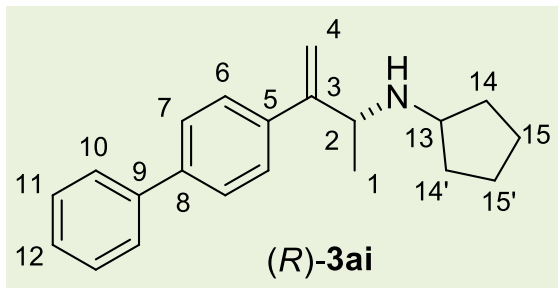

Synthesized at room temperature for $72 \mathrm{~h}$ following the general procedure using 4-(buta-1,3-dien-2-yl)-1,1'biphenyl 1a (51.6 mg, 0.25 mmol, 1.0 equiv), cyclopentylamine $2 \mathbf{i}(99 \mu \mathrm{L}, 1.00 \mathrm{mmol}, 4.0$ equiv), $\mathrm{Ni}(\operatorname{cod})_{2}(3.4 \mathrm{mg}, 0.0125 \mathrm{mmol}, 5 \mathrm{~mol} \%)$, $(R, R)$-BenzP* (4.2 mg, $0.015 \mathrm{mmol}, 6 \mathrm{~mol} \%$ ), 2,2,2-trifluoroethanol (19 $\mu \mathrm{L}, 0.25 \mathrm{mmol}, 1.0$ equiv) and mesitylene $(0.50 \mathrm{~mL}, 0.5 \mathrm{M})$. Consumption of 1a: $>99 \%$, conversion of 3ai: $75 \%$, r.r. $=4: 1$. Purification by flash chromatography over silica gel (pentane:EtOAc $=1: 1$ ) led to the desired product as a yellow viscous oil ( $55.7 \mathrm{mg}, 76 \%$ yield, $92 \%$ ee)

${ }^{1} \mathbf{H}$ NMR $\left(400 \mathrm{MHz}, \mathrm{CDCl}_{3}\right) \delta(\mathrm{ppm})=7.64-7.54(\mathrm{~m}, 4 \mathrm{H}, \mathrm{H}-7+\mathrm{H}-10), 7.50-7.41(\mathrm{~m}, 4 \mathrm{H}, \mathrm{H}-$ $6+H-11), 7.38-7.31(\mathrm{~m}, 1 \mathrm{H}, H-12), 5.32(\mathrm{~s}, 1 \mathrm{H}, H-4), 5.30(\mathrm{~s}, 1 \mathrm{H}, H-4), 3.80\left(\mathrm{q},{ }^{3} J_{\mathrm{HH}}=6.5\right.$ $\mathrm{Hz}, 1 \mathrm{H}, H-2), 3.23\left(\mathrm{p},{ }^{3} \mathrm{~J}_{\mathrm{HH}}=7.0 \mathrm{~Hz}, 1 \mathrm{H}, H-13\right), 1.95-1.83(\mathrm{~m}, 2 \mathrm{H}, \mathrm{H}-14), 1.76-1.64(\mathrm{~m}$, $2 \mathrm{H}, H-15), 1.58-1.47(\mathrm{~m}, 2 \mathrm{H}, H-15), 1.41-1.25(\mathrm{~m}, 3 \mathrm{H}, \mathrm{NH}+H-14), 1.24\left(\mathrm{~d},{ }^{3} J_{H H}=6.6 \mathrm{~Hz}\right.$, $3 \mathrm{H}, \mathrm{H}-1)$.

${ }^{13} \mathrm{C}\left\{{ }^{1} \mathrm{H}\right\}$ NMR $\left(100 \mathrm{MHz}, \mathrm{CDCl}_{3}\right) \delta(\mathrm{ppm})=152.1(C-3), 140.9(C-9), 140.4(C-5), 140.3(C-8)$, $128.9(\mathrm{CH}-11), 127.5(\mathrm{CH}-6), 127.4(\mathrm{CH}-12), 127.1(\mathrm{CH}-10), 127.0(\mathrm{CH}-7), 112.6\left(\mathrm{CH}_{2}-4\right)$, $57.2(\mathrm{CH}-13), 55.9(\mathrm{CH}-2), 33.7\left(\mathrm{CH}_{2}-14\right), 33.4\left(\mathrm{CH}_{2}-14\right), 24.2\left(\mathrm{CH}_{2}-15\right), 24.0\left(\mathrm{CH}_{2}-15\right), 22.3$ $\left(\mathrm{CH}_{3}-1\right)$.

HRMS $\left(\mathrm{ESI}^{+}\right)$: calculated $[\mathrm{M}+\mathrm{H}]^{+}$for $\mathrm{C}_{21} \mathrm{H}_{26} \mathrm{~N}^{+}: 292.2060$; found: 296.2106.

IR (neat) $\vee\left(\mathrm{cm}^{-1}\right)$ : 3030, 2953, 2865, 1625, 1601, 1486, 1447, 1367, 1148, 1084, 1007, 906, 841, 769, 739, 695.

HPLC: $92 \%$ ee, chiral stationary column: $\mathrm{OJ}-\mathrm{H}$, mobile phase: hexane/iPrOH $=97 / 3,1.0$ $\mathrm{mL} / \mathrm{min}, 254 \mathrm{~nm}, 30^{\circ} \mathrm{C}, \mathrm{t}_{\mathrm{R}}$ (minor) $=7.2 \mathrm{~min}, \mathrm{t}_{\mathrm{R}}$ (major) $=8.2 \mathrm{~min}$.

$[\alpha]^{20} \mathrm{D}=+1.0\left(c 1.0, \mathrm{CHCl}_{3}\right)$. 


\section{<Chromatogram>}

mAU

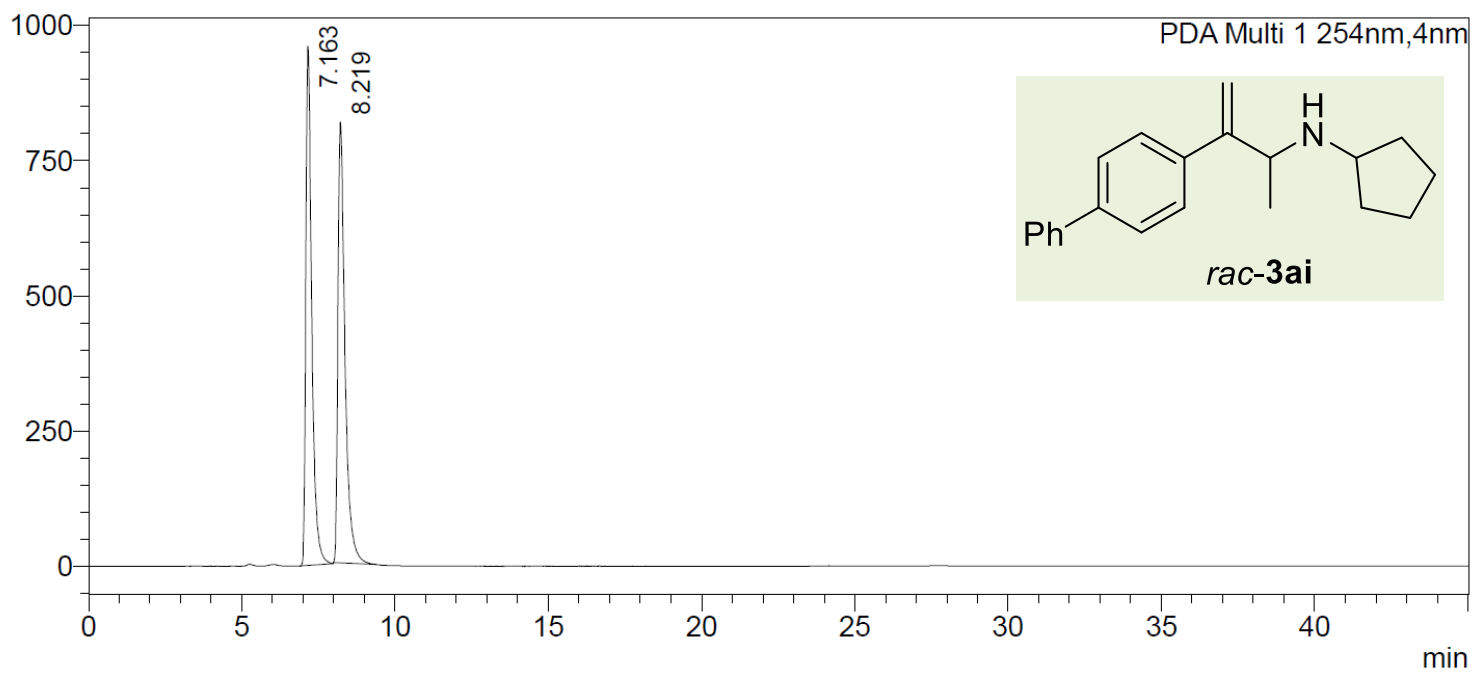

<Peak Table>

PDA Ch1 254nm

\begin{tabular}{|r|r|r|}
\hline Peak\# & Ret. Time & \multicolumn{1}{|c|}{ Area\% } \\
\hline 1 & 7.163 & 49.906 \\
\hline 2 & 8.219 & 50.094 \\
\hline Total & & 100.000 \\
\hline
\end{tabular}

\section{$<$ Chromatogram $>$}

mAU

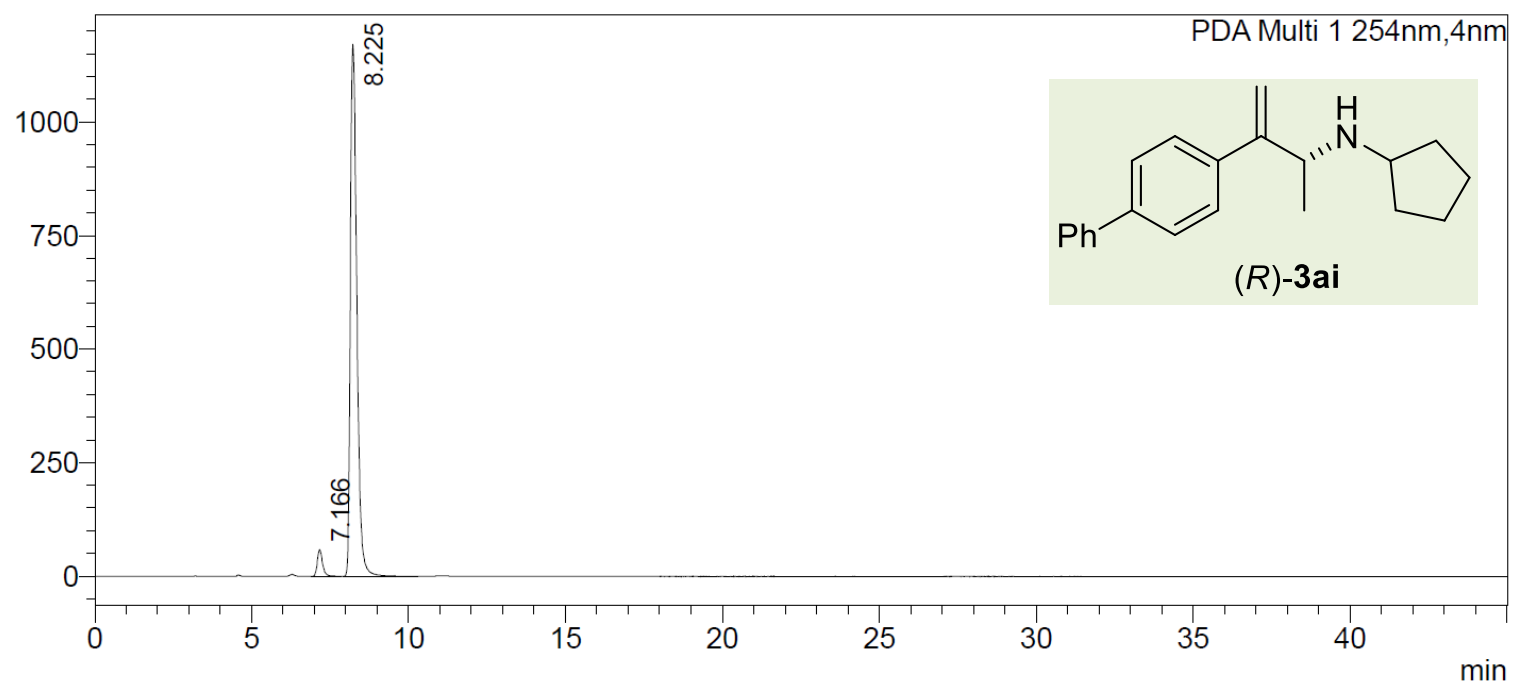

<Peak Table>

PDA Ch1 254nm

Peak\# Ret. Time

\begin{tabular}{|r|r|r|}
\hline 1 & 7.166 & Area $\%$ \\
\hline 2 & 8.225 & 9.866 \\
\hline Total & & 100.000 \\
\hline
\end{tabular}




\section{(R)-N-(3-([1,1'-Biphenyl]-4-yl)but-3-en-2-yl)cyclohexanamine 3aj}

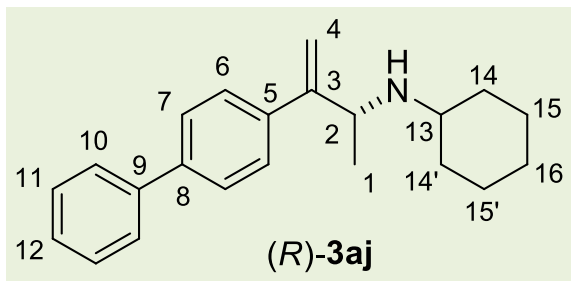

Synthesized at room temperature for $96 \mathrm{~h}$ following the general procedure using 4-(buta-1,3-dien-2-yl)-1,1'biphenyl 1a (51.6 mg, 0.25 mmol, 1.0 equiv), cyclohexylamine 2j (114 $\mu \mathrm{L}, 1.00 \mathrm{mmol}, 4.0$ equiv), $\mathrm{Ni}(\operatorname{cod})_{2}(3.4 \mathrm{mg}, 0.0125 \mathrm{mmol}, 5 \mathrm{~mol} \%),(R, R)-$ BenzP* $^{*}$ (4.2 mg, $0.015 \mathrm{mmol}, 6 \mathrm{~mol} \%$ ), 2,2,2-trifluoroethanol (19 $\mu \mathrm{L}, 0.25 \mathrm{mmol}, 1.0$ equiv) and mesitylene $(0.50 \mathrm{~mL}, 0.5 \mathrm{M})$. Consumption of 1a: $94 \%$, conversion of 3aj: $68 \%$, r.r. = 3:1. Purification by flash chromatography over silica gel (pentane:EtOAc $=2: 1$ ) led to the desired product as a colorless viscous oil $(50.2 \mathrm{mg}, 66 \%$ yield, $92 \%$ ee) .

${ }^{1} \mathrm{H}$ NMR $\left(400 \mathrm{MHz}, \mathrm{CDCl}_{3}\right) \delta(\mathrm{ppm})=7.66-7.54(\mathrm{~m}, 4 \mathrm{H}, \mathrm{H}-7+\mathrm{H}-10), 7.50-7.40(\mathrm{~m}, 4 \mathrm{H}, \mathrm{H}-$ $6+H-11), 7.39-7.30(\mathrm{~m}, 1 \mathrm{H}, H-12), 5.31\left(\mathrm{~d},{ }^{2} J_{H H}=0.8 \mathrm{~Hz}, 1 \mathrm{H}, H-4\right), 5.26(\mathrm{~s}, 1 \mathrm{H}, H-4), 3.92$ (q, $\left.{ }^{3} J_{H H}=6.5 \mathrm{~Hz}, 1 \mathrm{H}, H-2\right), 2.65-2.51(\mathrm{~m}, 1 \mathrm{H}, H-13), 2.05-1.93(\mathrm{~m}, 1 \mathrm{H}, H-14), 1.89-1.79$ (m, 1H, H-14), $1.78-1.67$ (m, 2H, $H-15+H-15), 1.66-1.56$ (m, 1H, $H-16), 1.32-1.04$ (m, $\left.6 \mathrm{H}, \mathrm{NH}+H-14+H-14^{\prime}+H-15+H-15^{\prime}+H-16\right), 1.22\left(\mathrm{~d},{ }^{3} J_{H H}=6.6 \mathrm{~Hz}, 3 \mathrm{H}, H-1\right)$.

${ }^{13} \mathrm{C}\left\{{ }^{1} \mathrm{H}\right\}$ NMR $\left(100 \mathrm{MHz}, \mathrm{CDCl}_{3}\right) \delta(\mathrm{ppm})=152.2(C-3), 140.9(C-9), 140.4(C-5), 140.2(C-8)$, $128.9(\mathrm{CH}-11), 127.5(\mathrm{CH}-6), 127.4(\mathrm{CH}-12), 127.1(\mathrm{CH}-10), 127.0(\mathrm{CH}-7), 112.5\left(\mathrm{CH}_{2}-4\right)$, $53.81(\mathrm{CH}-2$ or $\mathrm{CH}-13), 53.77(\mathrm{CH}-13$ or $\mathrm{CH}-2), 34.4\left(\mathrm{CH}_{2}-14\right), 33.7\left(\mathrm{CH}_{2}-14\right), 26.3\left(\mathrm{CH}_{2}-16\right)$, $25.5\left(\mathrm{CH}_{2}-15\right), 25.2\left(\mathrm{CH}_{2}-15\right), 22.5\left(\mathrm{CH}_{3}-1\right)$.

HRMS (ESI+): calculated $[\mathrm{M}+\mathrm{H}]^{+}$for $\mathrm{C}_{22} \mathrm{H}_{28} \mathrm{~N}^{+}: 306.2217$; found: 306.2249 .

IR (neat) $\vee\left(\mathrm{cm}^{-1}\right):$ 2924, 2851, 1601, 1486, 1447, 1368, 1276, 1261, 1132, 1084, 1007, 906, $842,765,749,695$.

HPLC: $92 \%$ ee, chiral stationary column: $\mathrm{OJ}-\mathrm{H}$, mobile phase: hexane/iPrOH $=97 / 3,1.0$ $\mathrm{mL} / \mathrm{min}, 254 \mathrm{~nm}, 30^{\circ} \mathrm{C}, \mathrm{t}_{\mathrm{R}}$ (minor) $=6.4 \mathrm{~min}, \mathrm{t}_{\mathrm{R}}$ (major) $=7.7 \mathrm{~min}$.

$[\alpha]^{20} \mathrm{D}=-5.6\left(c 1.0, \mathrm{CHCl}_{3}\right)$. 
<Chromatogram>

$\mathrm{mAU}$

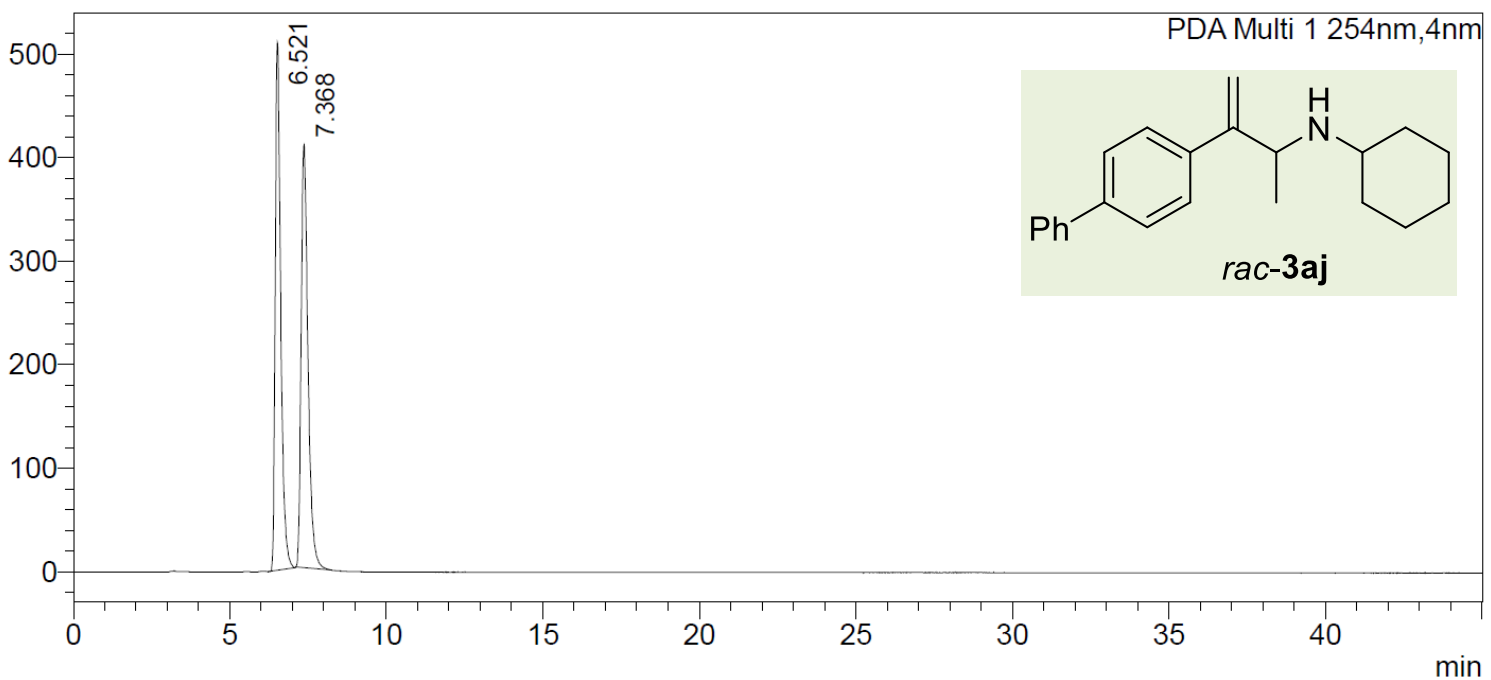

<Peak Table>

PDA Ch1 254nm

\begin{tabular}{|l|l|}
\hline Peak\# Ret. Time & Area\% \\
\hline
\end{tabular}

\begin{tabular}{|r|r|r|}
\hline 1 & 6.521 & 50.047 \\
\hline 2 & 7.368 & 49.953 \\
\hline Total & & 100.000 \\
\hline
\end{tabular}

\section{<Chromatogram>}

mAU

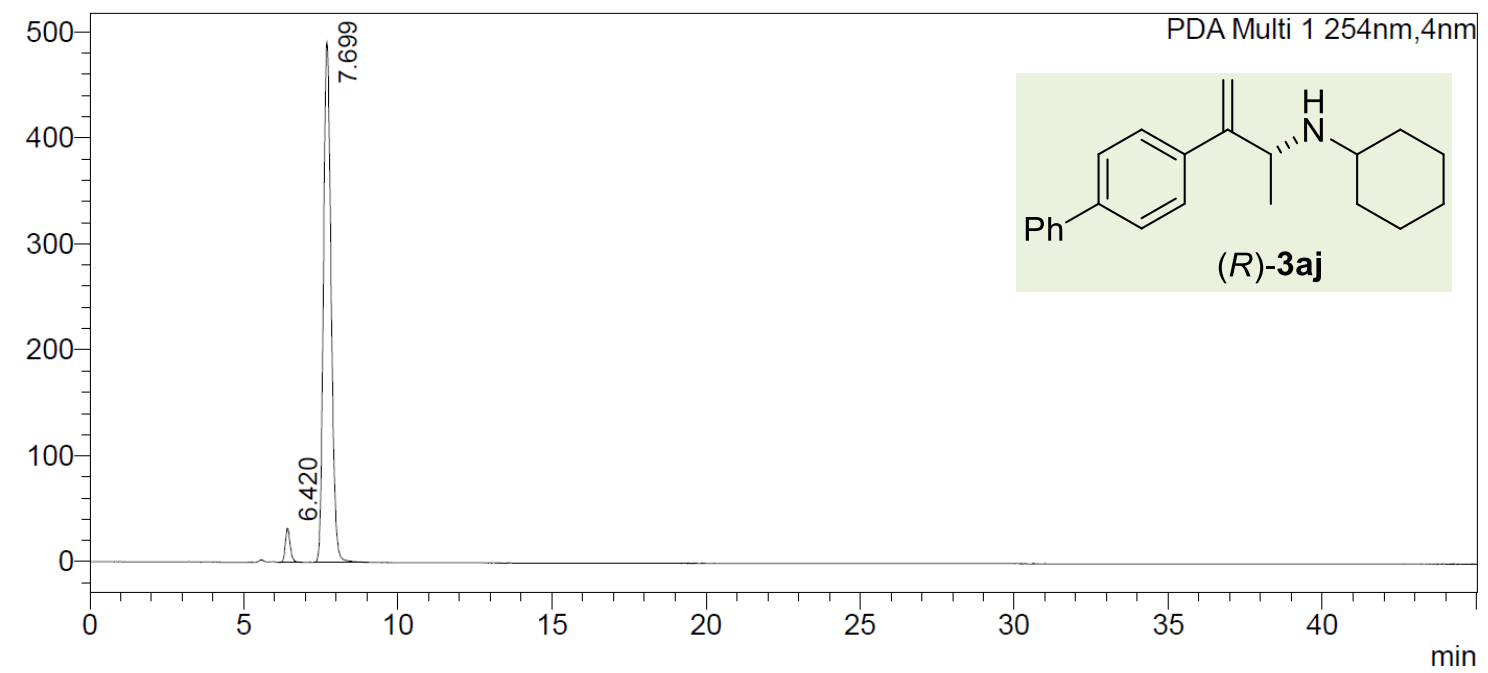

<Peak Table>

PDA Ch1 254nm

\begin{tabular}{|r|r|r|}
\hline Peak\# & Ret. Time & \multicolumn{1}{|c|}{ Area\% } \\
\hline 1 & 6.420 & 3.964 \\
\hline 2 & 7.699 & 96.036 \\
\hline Total & & 100.000 \\
\hline
\end{tabular}




\section{3-([1,1'-Biphenyl]-4-yl)-N-((S)-1-phenylethyl)but-3-en-2-amine 3ak+3al}

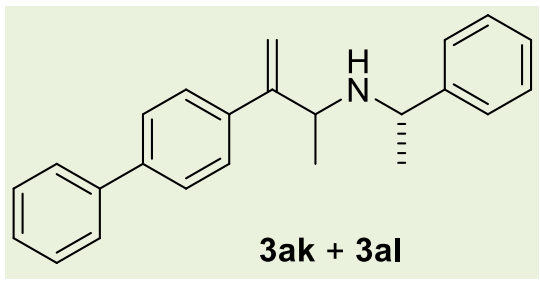

Synthesized at room temperature for $24 \mathrm{~h}$ following the general racemic procedure using 4-(buta-1,3-dien-2-yl)1,1'-biphenyl 1a (20.6 mg, $0.10 \mathrm{mmol}, 1.0$ equiv), (S)-1phenylethylamine 2k (52 $\mu \mathrm{L}, 1.00 \mathrm{mmol}, 4.0$ equiv), $\mathrm{Ni}(\mathrm{cod})_{2}$ (1.4 mg, $\left.0.005 \mathrm{mmol}, 5 \mathrm{~mol} \%\right), \mathrm{H}, \mathrm{H}-\mathrm{Cy}-\mathrm{Phox}(1.7$ $\mathrm{mg}, 0.005 \mathrm{mmol}, 5 \mathrm{~mol} \%)$, 2,2,2-trifluoroethanol (7.5 $\mu \mathrm{L}, 0.10 \mathrm{mmol}, 1.0$ equiv) and mesitylene $(0.2 \mathrm{~mL}, 0.5 \mathrm{M})$. Consumption of 1a: $>99 \%$, conversion of 3ak+3al: $86 \%$, r.r. > $19: 1$, d.r. $=1.05: 1$. Purification by flash chromatography over silica gel (Pentane:EtOAc $=$ 6:1) led to the desired product as a colorless viscous oil. Diasteroisomeric ratio (d.r.) was calculated by integration of non isochronic signals of ${ }^{1} \mathrm{H} N \mathrm{NM}$ of crude reaction mixture $(\delta=$ 3.60 ppm and 3.40 ppm).

\section{(R)-3-([1,1'-Biphenyl]-4-yl)-N-((S)-1-phenylethyl)but-3-en-2-amine 3ak}

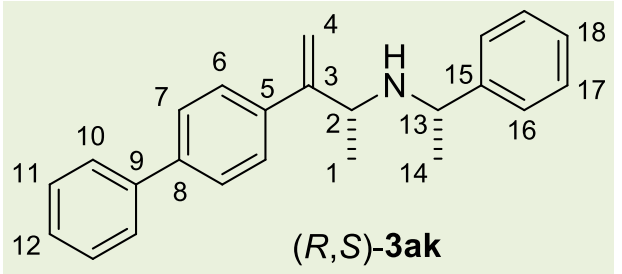

Synthesized at room temperature for $144 \mathrm{~h}$ following the general procedure using 4-(buta-1,3-dien-2-yl)1,1 '-biphenyl 1a (51.6 mg, $0.25 \mathrm{mmol}, 1.0$ equiv), (S)1-phenylethylamine $\mathbf{2 k}$ (129 $\mu \mathrm{L}, 1.00 \mathrm{mmol}, 4.0$ equiv), $\mathrm{Ni}(\mathrm{cod})_{2} \quad(3.4 \mathrm{mg}, 0.0125 \mathrm{mmol}, 5 \mathrm{~mol} \%),(R, R)-$ BenzP* $^{*}(4.2 \mathrm{mg}, 0.015 \mathrm{mmol}, 6 \mathrm{~mol} \%)$, 2,2,2-trifluoroethanol (19 $\mu \mathrm{L}, 0.25 \mathrm{mmol}, 1.0$ equiv) and mesitylene $(0.50 \mathrm{~mL}, 0.5 \mathrm{M})$. Consumption of 1a: $94 \%$, conversion of 3ak: $61 \%$, r.r. $=$ $3: 1$, d.r. $=19: 1$. Purification by flash chromatography over silica gel (Pentane:EtOAc = 6:1) led to the desired product as a colorless viscous oil ( $49.4 \mathrm{mg}, 60 \%$ yield, $19: 1$ d.r.).

${ }^{1} \mathbf{H}$ NMR $\left(400 \mathrm{MHz}, \mathrm{CDCl}_{3}\right) \delta(\mathrm{ppm})=7.56-7.49(\mathrm{~m}, 2 \mathrm{H}, \mathrm{H}-10), 7.47-7.41(\mathrm{~m}, 2 \mathrm{H}, \mathrm{H}-7)$, $7.40-7.33(\mathrm{~m}, 2 \mathrm{H}, H-11), 7.30-7.16(\mathrm{~m}, 8 \mathrm{H}, \mathrm{H}-6+\mathrm{H}-12+\mathrm{H}-16+\mathrm{H}-17+\mathrm{H}-18), 5.24\left(\mathrm{~d},{ }^{2} \mathrm{~J}_{\mathrm{HH}}=\right.$ $1.0 \mathrm{~Hz}, 1 \mathrm{H}, H-4), 5.21\left(\mathrm{~d},{ }^{2} \mathrm{~J}_{\mathrm{HH}}=1.0 \mathrm{~Hz}, 1 \mathrm{H}, H-4\right), 3.88\left(\mathrm{q},{ }^{3} J_{\mathrm{HH}}=6.6 \mathrm{~Hz}, 1 \mathrm{H}, H-13\right), 3.60(\mathrm{q}$, $\left.{ }^{3} J_{\mathrm{HH}}=6.3 \mathrm{~Hz}, 1 \mathrm{H}, \mathrm{H}-2\right), 1.41(\mathrm{~s}, 1 \mathrm{H}, \mathrm{NH}), 1.30\left(\mathrm{~d},{ }^{3} J_{\mathrm{HH}}=6.6 \mathrm{~Hz}, 3 \mathrm{H}, H-14\right), 1.18\left(\mathrm{~d},{ }^{3} J_{\mathrm{HH}}=\right.$ $6.5 \mathrm{~Hz}, 3 \mathrm{H}, \mathrm{H}-1)$.

${ }^{13} \mathrm{C}\left\{{ }^{1} \mathrm{H}\right\}$ NMR $\left(100 \mathrm{MHz}, \mathrm{CDCl}_{3}\right) \delta(\mathrm{ppm})=152.4(C-3), 145.8(C-15), 140.9(C-9), 140.29(C-$ 5), $140.26(\mathrm{C}-8), 128.9(\mathrm{CH}-11), 128.6(\mathrm{CH}-17), 127.5(\mathrm{CH}-6), 127.4(\mathrm{CH}-12), 127.1(\mathrm{CH}-$ 10), 127.05 ( $\mathrm{CH}-18), 126.99(\mathrm{CH}-7), 126.8(\mathrm{CH}-16), 112.5\left(\mathrm{CH}_{2}-4\right), 55.0(\mathrm{CH}-13), 53.5(\mathrm{CH}-$ 2), $24.0\left(\mathrm{CH}_{3}-14\right), 20.7\left(\mathrm{CH}_{3}-1\right)$.

HRMS $\left(\mathrm{ESI}^{+}\right)$: calculated $[\mathrm{M}+\mathrm{H}]^{+}$for $\mathrm{C}_{24} \mathrm{H}_{26} \mathrm{~N}^{+}: 328.2060$; found: 328.2081 .

IR (neat) $v\left(\mathrm{~cm}^{-1}\right):$ 3060, 3028, 2966, 2926, 2864, 1624, 1601, 1487, 1449, 1369, 1130, 1076, 1007, 907, 843, 766, 740, 697.

$[\alpha]^{20} \mathrm{D}=+23.5\left(\mathrm{c} 1.0, \mathrm{CHCl}_{3}\right)$. 


\section{(R)-3-([1,1'-Biphenyl]-4-yl)-N-((R)-1-phenylethyl)but-3-en-2-amine 3al}

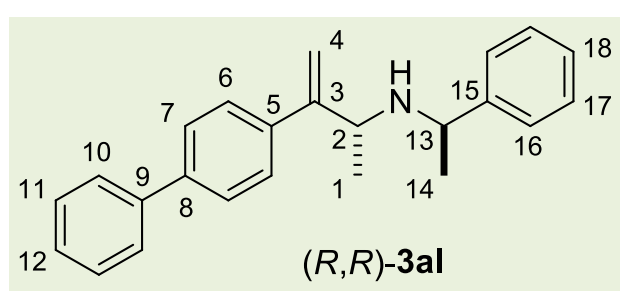

Synthesized at room temperature for $144 \mathrm{~h}$ following the general procedure using 4-(buta-1,3-dien-2-yl)1,1 '-biphenyl 1a (51.6 mg, $0.25 \mathrm{mmol}, 1.0$ equiv), $(R)$ 1-phenylethylamine $2 \mathbf{l}(129 \mu \mathrm{L}, 1.00 \mathrm{mmol}, 4.0$ equiv), $\mathrm{Ni}(\mathrm{cod})_{2} \quad(3.4 \mathrm{mg}, 0.0125 \mathrm{mmol}, 5 \mathrm{~mol} \%),(R, R)-$ BenzP* $^{*}(4.2 \mathrm{mg}, 0.015 \mathrm{mmol}, 6 \mathrm{~mol} \%)$, 2,2,2-trifluoroethanol (19 $\mu \mathrm{L}, 0.25 \mathrm{mmol}, 1.0$ equiv) and mesitylene $(0.50 \mathrm{~mL}, 0.5 \mathrm{M})$. Consumption of $1 \mathrm{a}: 84 \%$, conversion of $3 \mathrm{al}: 56 \%$, r.r. $=3: 1$, d.r. $=17: 1$. Purification by flash chromatography over silica gel (Pentane:EtOAc $=8: 1$ ) led to the desired product as a colorless viscous oil (44.2 $\mathrm{mg}, 54 \%$ yield, $17: 1$ d.r.).

${ }^{1} \mathbf{H}$ NMR $\left(400 \mathrm{MHz}, \mathrm{CDCl}_{3}\right) \delta(\mathrm{ppm})=7.57-7.49(\mathrm{~m}, 2 \mathrm{H}, \mathrm{H}-10), 7.48-7.41(\mathrm{~m}, 2 \mathrm{H}, \mathrm{H}-7)$, $7.40-7.33(\mathrm{~m}, 2 \mathrm{H}, \mathrm{H}-11), 7.31-7.15(\mathrm{~m}, 8 \mathrm{H}, \mathrm{H}-6+\mathrm{H}-12+\mathrm{H}-16+H-17+H-18), 5.24\left(\mathrm{~d},{ }^{2} \mathrm{~J}_{\mathrm{HH}}=\right.$ $0.8 \mathrm{~Hz}, 1 \mathrm{H}, H-4), 5.13(\mathrm{~s}, 1 \mathrm{H}, H-4), 3.86\left(\mathrm{q},{ }^{3} \mathrm{~J}_{\mathrm{HH}}=6.6 \mathrm{~Hz}, 1 \mathrm{H}, H-13\right), 3.40\left(\mathrm{q},{ }^{3} \mathrm{~J}_{\mathrm{HH}}=6.6 \mathrm{~Hz}\right.$, $1 \mathrm{H}, H-2), 1.44(\mathrm{~s}, 1 \mathrm{H}, \mathrm{NH}), 1.28\left(\mathrm{~d},{ }^{3} \mathrm{~J}_{\mathrm{HH}}=6.7 \mathrm{~Hz}, 3 \mathrm{H}, H-14\right), 1.04\left(\mathrm{~d},{ }^{3} \mathrm{~J}_{\mathrm{HH}}=6.6 \mathrm{~Hz}, 3 \mathrm{H}, H-1\right)$. ${ }^{13} \mathrm{C}\left\{{ }^{1} \mathrm{H}\right\}$ NMR $\left(100 \mathrm{MHz}, \mathrm{CDCl}_{3}\right) \delta(\mathrm{ppm})=151.8(C-3), 146.2(C-15), 140.9(C-9), 140.5(C-$ 5), $140.2(\mathrm{C}-8), 128.9(\mathrm{CH}-11), 128.6(\mathrm{CH}-17), 127.6(\mathrm{CH}-6), 127.4(\mathrm{CH}-12), 127.1(\mathrm{CH}-10)$, $127.05(\mathrm{CH}-18), 126.98(\mathrm{CH}-7), 126.9(\mathrm{CH}-16), 112.5\left(\mathrm{CH}_{2}-4\right), 55.2(\mathrm{CH}-13), 55.0(\mathrm{CH}-2)$, $25.1\left(\mathrm{CH}_{3}-14\right)$, $22.9\left(\mathrm{CH}_{3}-1\right)$.

HRMS $\left(\mathrm{ESI}^{+}\right)$: calculated $[\mathrm{M}+\mathrm{H}]^{+}$for $\mathrm{C}_{24} \mathrm{H}_{26} \mathrm{~N}^{+}: 328.2060$; found: 328.2081.

IR (neat) $v\left(\mathrm{~cm}^{-1}\right):$ 3060, 3028, 2962, 2924, 2864, 1625, 1601, 1487, 1448, 1369, 1133, 1079, 1007, 908, 842, 764, 740, 697.

$[\alpha]^{20} \mathrm{D}=+28.8\left(c 1.0, \mathrm{CHCl}_{3}\right)$. 


\section{(R)-1-(3-([1,1'-Biphenyl]-4-yl)but-3-en-2-yl)pyrrolidine 3am}

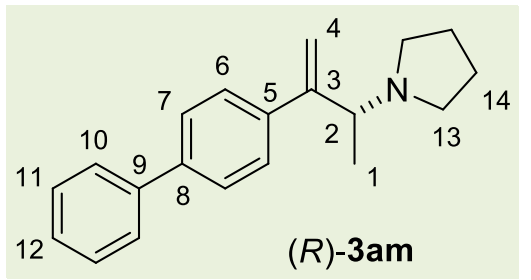

Synthesized at room temperature for $72 \mathrm{~h}$ using diphenylphosphinic acid as additive following the general procedure using 4-(buta-1,3-dien-2-yl)-1,1'-biphenyl 1a (51.6 $\mathrm{mg}, 0.25 \mathrm{mmol}, 1.0$ equiv), pyrrolidine $2 \mathrm{~m}$ (84 $\mu \mathrm{L}, 1.00$ mmol, 4.0 equiv), $\mathrm{Ni}(\mathrm{cod})_{2}$ (3.4 mg, $\left.0.0125 \mathrm{mmol}, 5 \mathrm{~mol} \%\right)$, $(R, R)$-BenzP* (4.2 mg, $0.015 \mathrm{mmol}, 6 \mathrm{~mol} \%)$, diphenylphosphinic acid (10.9 mg, $0.05 \mathrm{mmol}$, 0.20 equiv) and mesitylene ( $0.50 \mathrm{~mL}, 0.5 \mathrm{M})$. Consumption of 1a: $>99 \%$, conversion of 3am: $67 \%, r . r .=1.9: 1$. Purification by flash chromatography over silica gel (Pentane:EtOAc = 2:1) led to the desired product as a colorless oil ( $44.5 \mathrm{mg}, 64 \%$ yield, $95 \%$ ee).

${ }^{1} \mathrm{H}$ NMR $\left(400 \mathrm{MHz}, \mathrm{CDCl}_{3}\right) \delta(\mathrm{ppm})=7.67-7.59(\mathrm{~m}, 4 \mathrm{H}, \mathrm{H}-6+\mathrm{H}-10), 7.58-7.53(\mathrm{~m}, 2 \mathrm{H}, \mathrm{H}-$ 7), $7.48-7.39(\mathrm{~m}, 2 \mathrm{H}, H-11), 7.34$ (t, $\left.{ }^{3} \mathrm{~J}_{\mathrm{HH}}=7.3 \mathrm{~Hz}, 1 \mathrm{H}, H-12\right), 5.34$ (s, 2H, $\left.H-4\right), 3.19$ (q, $\left.{ }^{3} J_{H H}=6.5 \mathrm{~Hz}, 1 \mathrm{H}, H-2\right), 2.70-2.54(\mathrm{~m}, 4 \mathrm{H}, H-13), 1.86-1.75(\mathrm{~m}, 4 \mathrm{H}, H-14), 1.27\left(\mathrm{~d},{ }^{3} J_{H H}=\right.$ $6.5 \mathrm{~Hz}, 3 \mathrm{H}, \mathrm{H}-1)$.

${ }^{13} \mathrm{C}\left\{{ }^{1} \mathrm{H}\right\}$ NMR $\left(100 \mathrm{MHz}, \mathrm{CDCl}_{3}\right) \delta(\mathrm{ppm})=151.8(C-3), 141.1(C-9), 140.4(C-5), 140.2(C-8)$, $128.9(\mathrm{CH}-11), 127.7(\mathrm{CH}-6), 127.3(\mathrm{CH}-12), 127.1(\mathrm{CH}-10), 126.9(\mathrm{CH}-7), 114.2\left(\mathrm{CH}_{2}-4\right)$, $65.9(\mathrm{CH}-2), 52.8\left(\mathrm{CH}_{2}-13\right), 23.7\left(\mathrm{CH}_{2}-14\right), 21.0\left(\mathrm{CH}_{3}-1\right)$.

HRMS (ESI+): calculated [M+H] ${ }^{+}$for $\mathrm{C}_{20} \mathrm{H}_{24} \mathrm{~N}^{+}:$:278.1904; found: 278.2004 .

IR (neat) $v\left(\mathrm{~cm}^{-1}\right): 3029,2967,2927,2873,2782,1678,1624,1601,1486,1449,1370,1314$, 1196, 1142, 1076, 1007, 906, 843, 770, 741, 695.

HPLC: $95 \%$ ee, chiral stationary column: $\mathrm{OJ}-\mathrm{H}$, mobile phase: hexane/iPrOH $=98 / 2,1.0$ $\mathrm{mL} / \mathrm{min}, 254 \mathrm{~nm}, 30^{\circ} \mathrm{C}, \mathrm{t}_{\mathrm{R}}($ minor $)=6.7 \mathrm{~min}, \mathrm{t}_{\mathrm{R}}$ (major) $=7.5 \mathrm{~min}$.

$[\alpha]^{20} \mathrm{D}=+49.7\left(\mathrm{c} 1.0, \mathrm{CHCl}_{3}\right)$. 


\section{<Chromatogram>}

mAU

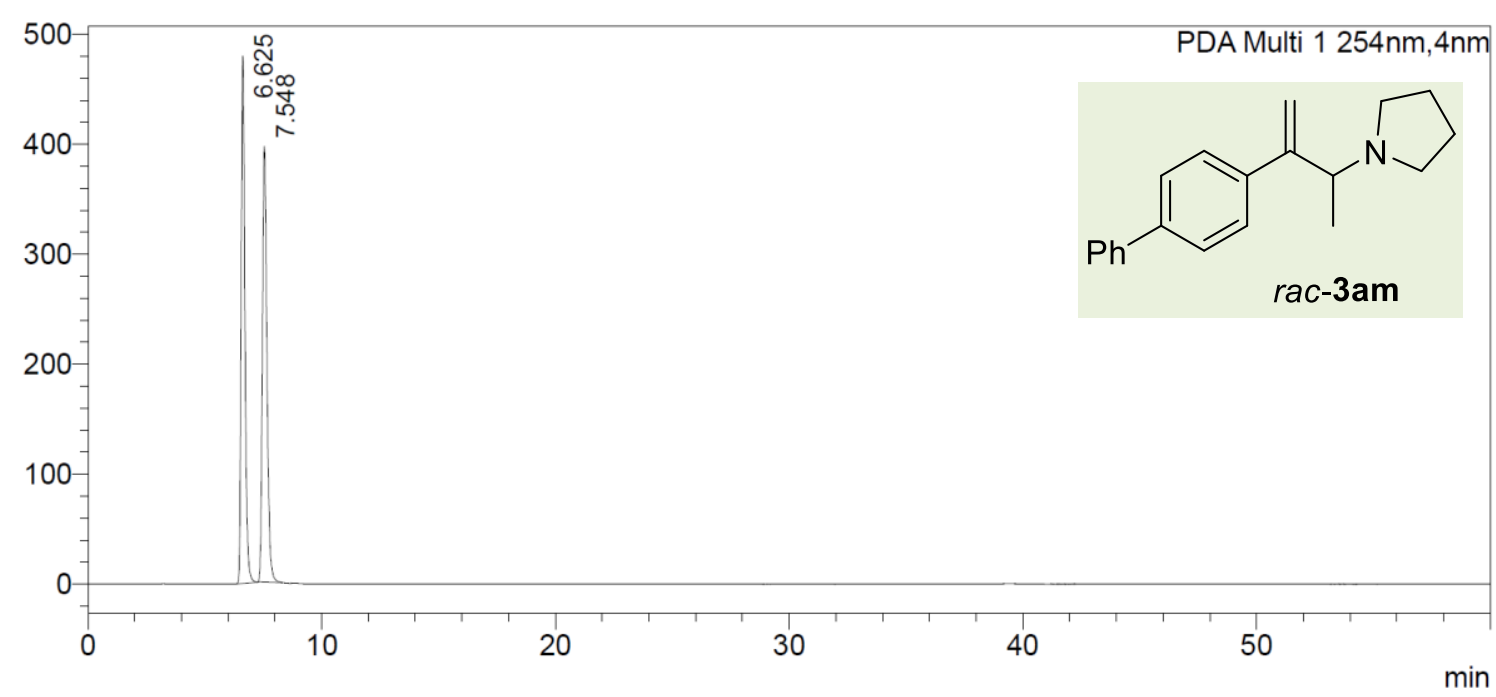

<Peak Table>

PDA Ch1 254nm

\begin{tabular}{|r|r|r|}
\hline Peak\# & Ret. Time & \multicolumn{1}{|c|}{ Area $\%$} \\
\hline 1 & 6.625 & 49.956 \\
\hline 2 & 7.548 & 50.044 \\
\hline Total & & 100.000 \\
\hline
\end{tabular}

<Chromatogram>

mAU

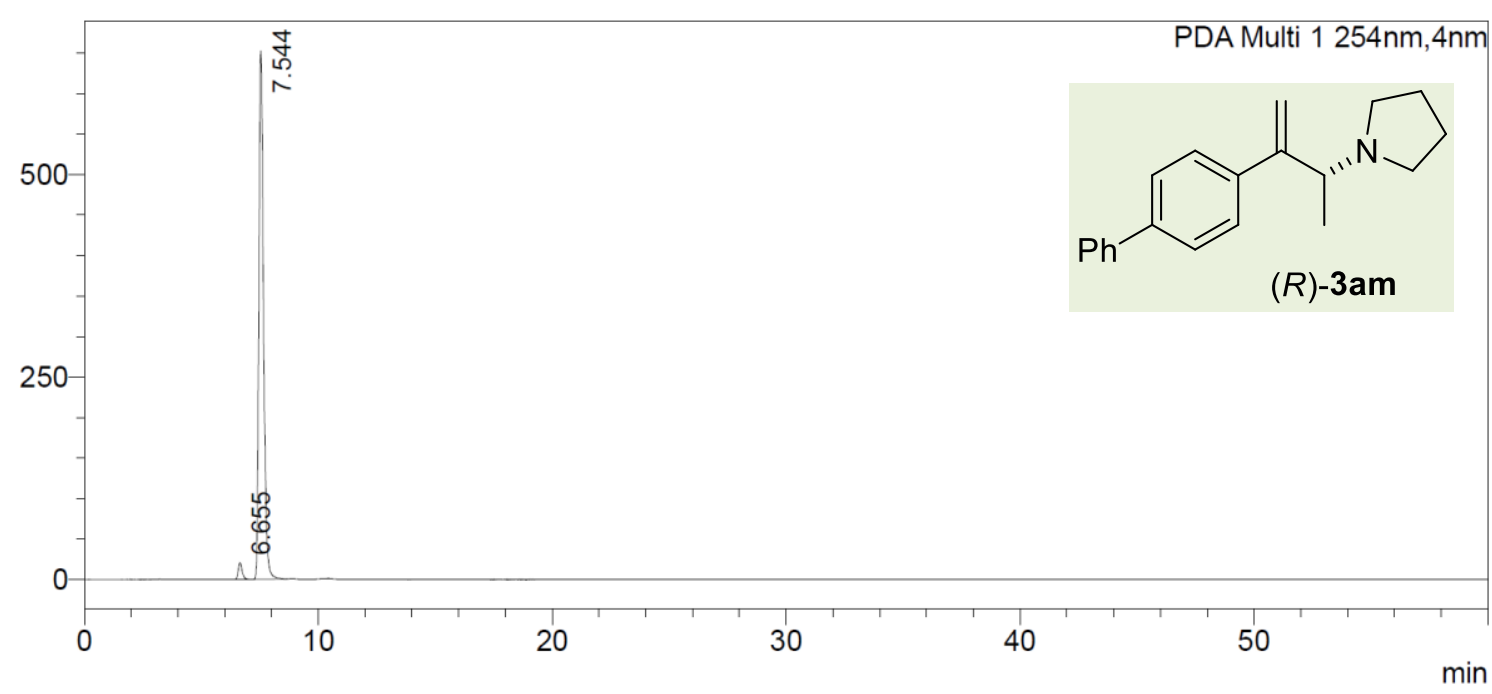

<Peak Table>

PDA Ch1 254nm

\begin{tabular}{|l|l|}
\hline Peak\# Ret. Time & Area\% \\
\hline
\end{tabular}

\begin{tabular}{|r|r|r|}
\hline 1 & 6.655 & 2.543 \\
\hline 2 & 7.544 & 97.457 \\
\hline Total & & 100.000 \\
\hline
\end{tabular}




\section{(R)-1-(3-([1,1'-Biphenyl]-4-yl)but-3-en-2-yl)piperidine 3an}

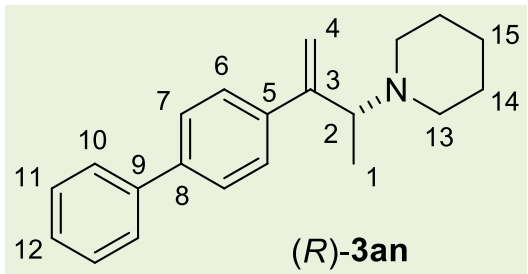

(R)-3an

Synthesized at room temperature for $72 \mathrm{~h}$ using diphenylphosphinic acid as additive following the general procedure using 4-(buta-1,3-dien-2-yl)-1,1'-biphenyl 1a (51.6 mg, 0.25 mmol, 1.0 equiv), piperidine $2 \mathrm{n}$ (99 $\mu \mathrm{L}, 1.00$ mmol, 4.0 equiv), $\mathrm{Ni}(\mathrm{cod})_{2}$ (3.4 mg, $\left.0.0125 \mathrm{mmol}, 5 \mathrm{~mol} \%\right)$, $(R, R)$-BenzP* (4.2 mg, $0.015 \mathrm{mmol}, 6 \mathrm{~mol} \%)$, diphenylphosphinic acid (10.9 mg, $0.05 \mathrm{mmol}$, 0.2 equiv) and mesitylene $(0.50 \mathrm{~mL}, 0.5 \mathrm{M})$. Consumption of 1a: $>99 \%$, conversion of 3an: $50 \%, r . r .=1.0: 1$. Purification by flash chromatography over silica gel (Pentane:EtOAc $=6: 1$ ) led to the desired product as a colorless viscous oil ( $34.9 \mathrm{mg}, 48 \%$ yield, $>95 \%$ ee) .

${ }^{1} \mathbf{H}$ NMR $\left(400 \mathrm{MHz}, \mathrm{CDCl}_{3}\right) \delta(\mathrm{ppm})=7.68-7.58(\mathrm{~m}, 4 \mathrm{H}, \mathrm{H}-6+\mathrm{H}-10), 7.58-7.51(\mathrm{~m}, 2 \mathrm{H}, \mathrm{H}-$ 7), $7.48-7.40(\mathrm{~m}, 2 \mathrm{H}, H-11), 7.34\left(\mathrm{t},{ }^{3} \mathrm{~J}_{\mathrm{HH}}=7.3 \mathrm{~Hz}, 1 \mathrm{H}, H-12\right), 5.39(\mathrm{~s}, 1 \mathrm{H}, H-4), 5.25(\mathrm{~s}, 1 \mathrm{H}$, $H-4), 3.39\left(\mathrm{q},{ }^{3} \mathrm{JHH}_{\mathrm{H}}=6.6 \mathrm{~Hz}, 1 \mathrm{H}, H-2\right), 2.66-2.35(\mathrm{~m}, 4 \mathrm{H}, H-13), 1.62-1.49(\mathrm{~m}, 4 \mathrm{H}, H-14)$, $1.48-1.39(\mathrm{~m}, 2 \mathrm{H}, H-15), 1.21\left(\mathrm{~d},{ }^{3} \mathrm{~J}_{\mathrm{HH}}=6.6 \mathrm{~Hz}, 3 \mathrm{H}, H-1\right)$.

${ }^{13} \mathrm{C}\left\{{ }^{1} \mathrm{H}\right\}$ NMR $\left(100 \mathrm{MHz}, \mathrm{CDCl}_{3}\right) \delta(\mathrm{ppm})=150.8(C-3), 141.1(C-5+C-9), 140.0(C-8), 128.9$ $(\mathrm{CH}-11), 127.7(\mathrm{CH}-6), 127.3(\mathrm{CH}-12), 127.1(\mathrm{CH}-10), 126.8(\mathrm{CH}-7), 114.0\left(\mathrm{CH}_{2}-4\right), 64.6$ (CH-2), $51.3\left(\mathrm{CH}_{2}-13\right), 26.6\left(\mathrm{CH}_{2}-14\right), 25.0\left(\mathrm{CH}_{2}-15\right), 16.4\left(\mathrm{CH}_{3}-1\right)$.

HRMS (ESI+): calculated [M+H] $]^{+}$for $\mathrm{C}_{21} \mathrm{H}_{26} \mathrm{~N}^{+}:$292.2060; found: 292.2176 .

IR (neat) $v\left(\mathrm{~cm}^{-1}\right): 3032,2929,2852,2795,2751,1622,1601,1486,1445,1373,1321,1153$, 1116, 1036, 1007, 947, 905, 842, 768, 739, 695.

SFC: $>95 \%$ ee, chiral stationary phase: AD column, gradient elution from $30 \%$ to $40 \% \mathrm{MeOH}$ (12 $\mathrm{min}), 3 \mathrm{ml} / \mathrm{min}, 210 \mathrm{~nm}, \mathrm{t}_{\mathrm{R}}($ minor $)=5.2 \mathrm{~min}, \mathrm{t}_{\mathrm{R}}($ major $)=5.8 \mathrm{~min}$.

$[\alpha]^{20} \mathrm{D}=+38.6\left(\mathrm{c} 1.0, \mathrm{CHCl}_{3}\right)$. 


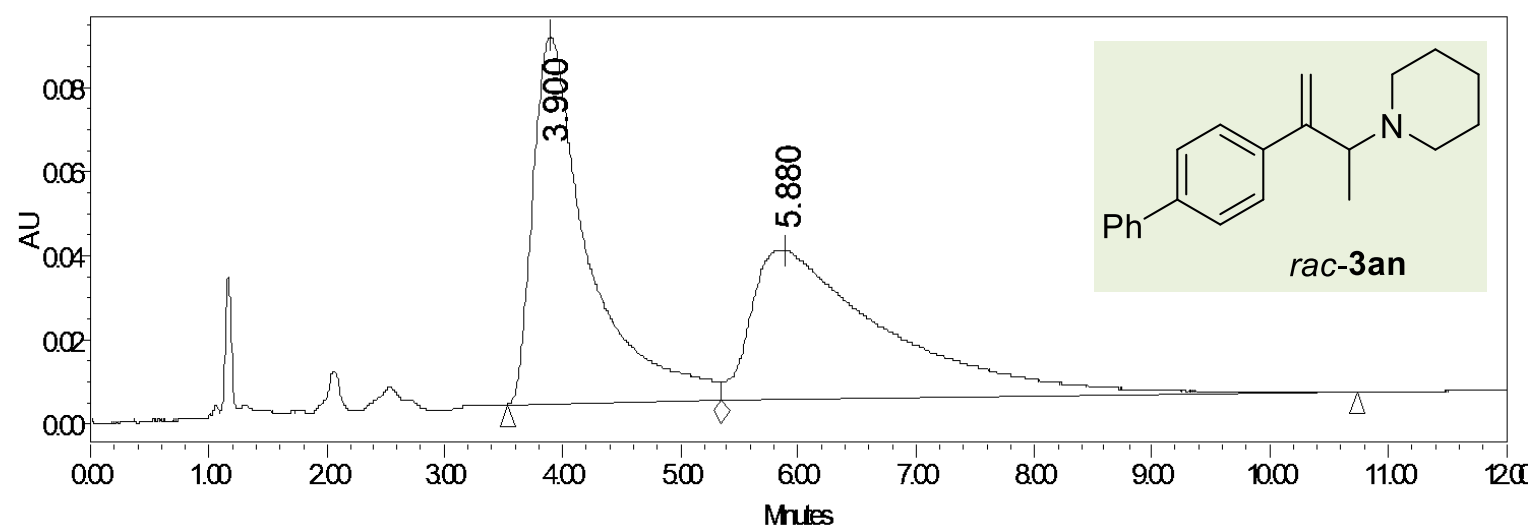

\begin{tabular}{|c|c|c|c|}
\hline & $\begin{array}{c}\text { Retention } \\
\text { Time }\end{array}$ & Area & \% Area \\
\hline 1 & 3.900 & 2873903 & 49.78 \\
\hline 2 & 5.880 & 2899812 & 50.22 \\
\hline
\end{tabular}

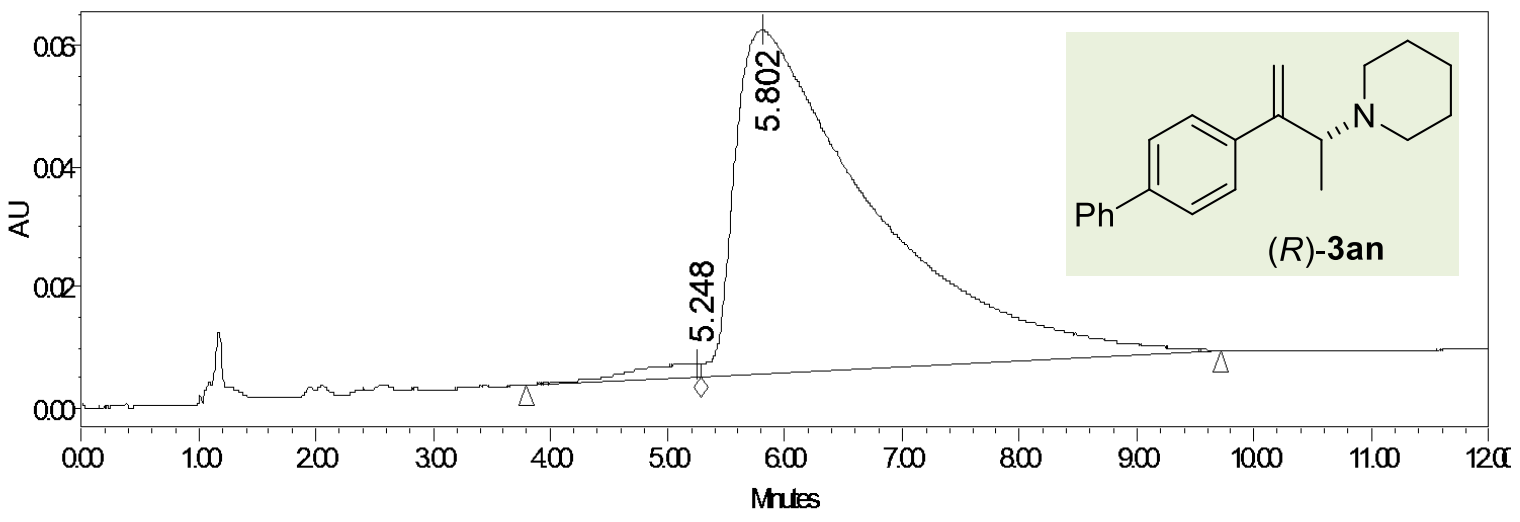

\begin{tabular}{|c|c|c|c|}
\hline & $\begin{array}{c}\text { Retention } \\
\text { Time }\end{array}$ & Area & $\%$ Area \\
\hline 1 & 5.248 & 84592 & 1.79 \\
\hline 2 & 5.802 & 4644243 & 98.21 \\
\hline
\end{tabular}




\section{(R)-4-(3-([1,1'-Biphenyl]-4-yl)but-3-en-2-yl)morpholine 3ao}

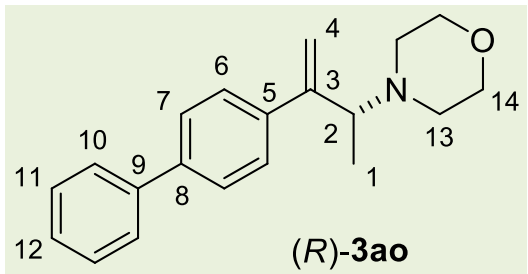

(R)-3ao

Synthesized at room temperature for $24 \mathrm{~h}$ using diphenylphosphinic acid as additive following the general procedure using 4-(buta-1,3-dien-2-yl)-1,1'-biphenyl 1a (51.6 mg, $0.25 \mathrm{mmol}, 1.0$ equiv), morpholine 20 (88 $\mu \mathrm{L}$, $1.00 \mathrm{mmol}, 4.0$ equiv), $\mathrm{Ni}(\mathrm{cod})_{2}$ (3.4 mg, $0.0125 \mathrm{mmol}, 5$ mol\%), (R,R)-BenzP* (4.2 mg, $0.015 \mathrm{mmol}, 6 \mathrm{~mol} \%$ ), diphenylphosphinic acid (10.9 mg, 0.05 mmol, 0.2 equiv) and mesitylene $(0.50 \mathrm{~mL}, 0.5 \mathrm{M})$. Consumption of $1 \mathrm{a}$ : $>99 \%$, conversion of 3ao: $56 \%$, r.r. $=1.2: 1$. Purification by flash chromatography over silica gel (Pentane:EtOAc $=$ $6: 1)$ led to the desired product as a colorless viscous oil ( $40.2 \mathrm{mg}, 55 \%$ yield, $95 \%$ ee).

${ }^{1} \mathrm{H}$ NMR $\left(400 \mathrm{MHz}, \mathrm{CDCl}_{3}\right) \delta(\mathrm{ppm})=7.70-7.62(\mathrm{~m}, 4 \mathrm{H}, \mathrm{H}-6+\mathrm{H}-10), 7.62-7.56(\mathrm{~m}, 2 \mathrm{H}, \mathrm{H}-$ 7), $7.51-7.43(\mathrm{~m}, 2 \mathrm{H}, H-11), 7.41-7.34(\mathrm{~m}, 1 \mathrm{H}, H-12), 5.45\left(\mathrm{~d},{ }^{2} J_{H H}=1.2 \mathrm{~Hz}, 1 \mathrm{H}, H-4\right)$, $5.31(\mathrm{~s}, 1 \mathrm{H}, H-4), 3.82-3.68(\mathrm{~m}, 4 \mathrm{H}, H-14), 3.36\left(\mathrm{q},{ }^{3} \mathrm{~J}_{\mathrm{HH}}=6.5 \mathrm{~Hz}, 1 \mathrm{H}, H-2\right), 2.68-2.51(\mathrm{~m}$, $4 \mathrm{H}, H-13), 1.25\left(\mathrm{~d},{ }^{3} \mathrm{~J}_{\mathrm{HH}}=6.6 \mathrm{~Hz}, 3 \mathrm{H}, H-1\right)$.

${ }^{13} \mathbf{C}\left\{{ }^{1} \mathrm{H}\right\}$ NMR $\left(100 \mathrm{MHz}, \mathrm{CDCl}_{3}\right) \delta(\mathrm{ppm})=149.9(C-3), 141.0(C-9), 140.3(C-5+C-8), 128.9$ $(\mathrm{CH}-11), 127.6(\mathrm{CH}-6), 127.4(\mathrm{CH}-12), 127.1(\mathrm{CH}-10), 126.9(\mathrm{CH}-7), 114.9\left(\mathrm{CH}_{2}-4\right), 67.5$ $\left(\mathrm{CH}_{2}-14\right), 65.1(\mathrm{CH}-2), 51.0\left(\mathrm{CH}_{2}-13\right), 16.8\left(\mathrm{CH}_{3}-1\right)$.

HRMS (ESI+): calculated [M+H] ${ }^{+}$for $\mathrm{C}_{20} \mathrm{H}_{24} \mathrm{NO}^{+}:$294.1853; found: 294.1896.

IR (neat) $\vee\left(\mathrm{cm}^{-1}\right): 3030,2958,2851,2805,1623,1600,1487,1449,1373,1320,1142,1117$, 1071, 1008, 955, 911, 845, 771, 741, 697.

HPLC: $95 \%$ ee, chiral stationary column: $\mathrm{OJ}-\mathrm{H}$, mobile phase: hexane/iPrOH $=95 / 5,1.0$ $\mathrm{mL} / \mathrm{min}, 254 \mathrm{~nm}, 30^{\circ} \mathrm{C}, \mathrm{t}_{\mathrm{R}}$ (minor) $=21.6 \mathrm{~min}, \mathrm{t}_{\mathrm{R}}$ (major) $=30.9 \mathrm{~min}$.

$[\alpha]^{20} \mathrm{D}=+52.4\left(c 1.0, \mathrm{CHCl}_{3}\right)$. 
<Chromatogram>

mAU

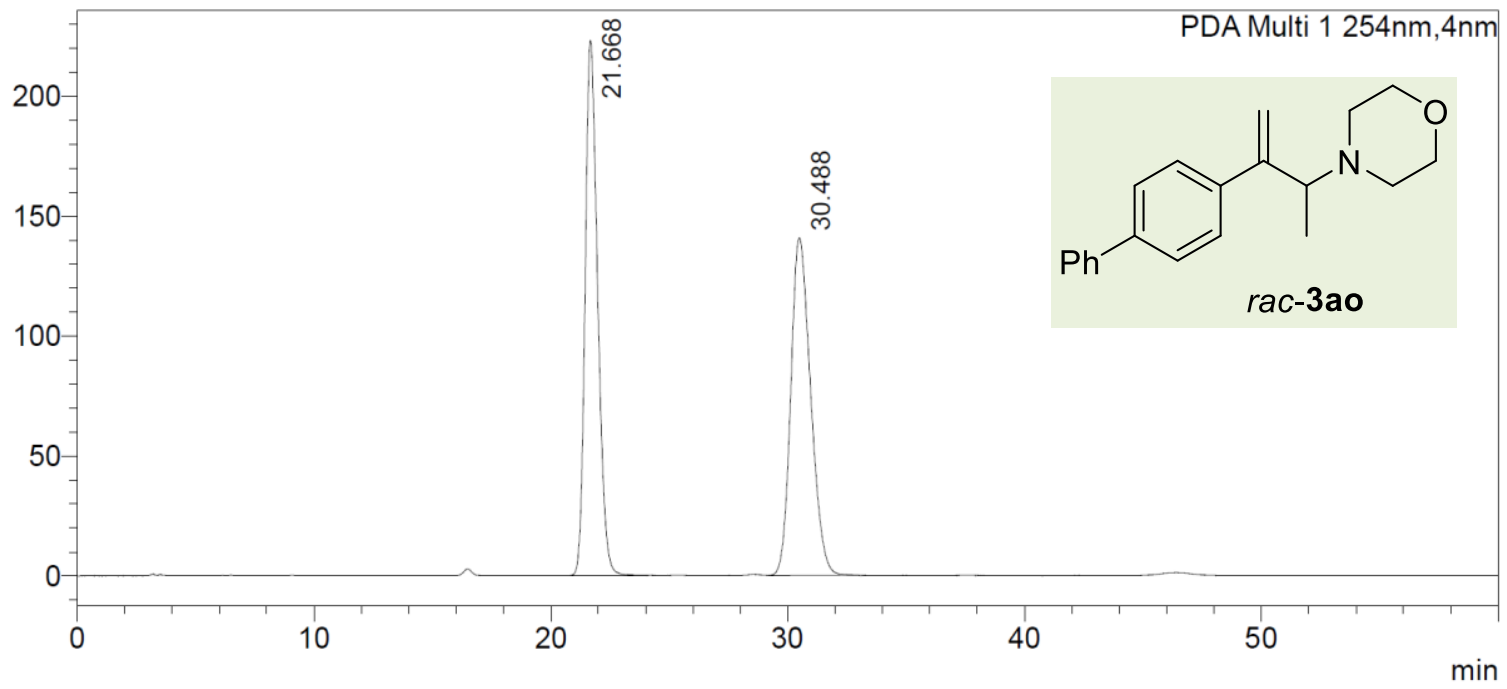

<Peak Table>

PDA Ch1 254nm

Peak\# Ret. Time $\quad$ Area\%

\begin{tabular}{|r|r|r|}
\hline 1 & 21.668 & 49.916 \\
\hline 2 & 30.488 & 50.084 \\
\hline Total & & 100.000 \\
\hline
\end{tabular}

<Chromatogram >

mAU

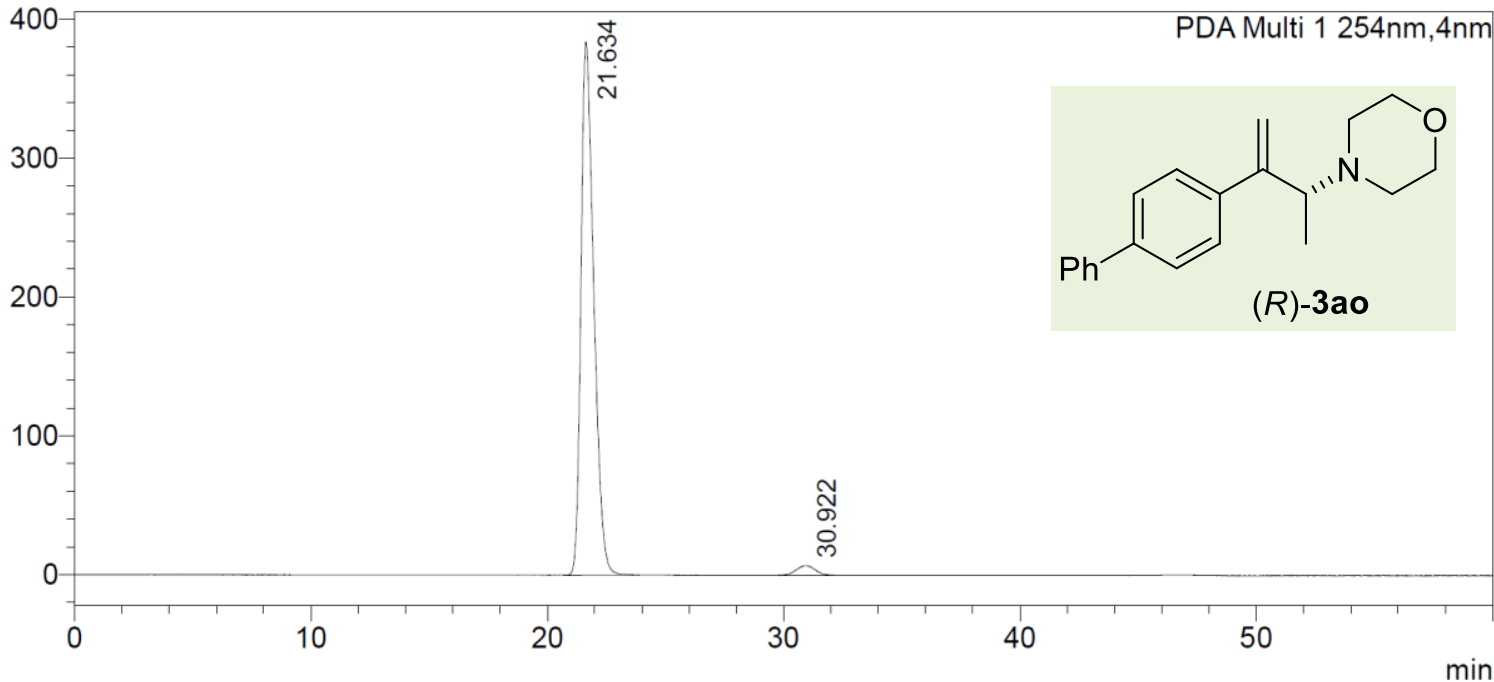

<Peak Table>

PDA Ch1 254nm

Peak\# Ret. Time Area\%

\begin{tabular}{|r|r|r|}
\hline 1 & 21.634 & 97.329 \\
\hline 2 & 30.922 & 2.671 \\
\hline Total & & 100.000 \\
\hline
\end{tabular}




\section{(R)-3-([1,1'-Biphenyl]-4-yl)-N-allylbut-3-en-2-amine 3ap}

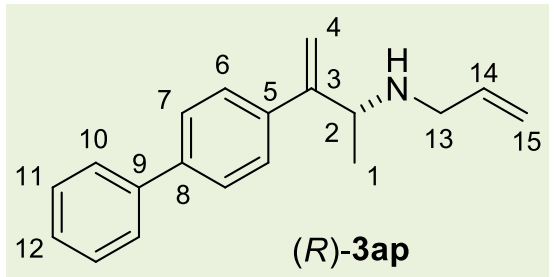

Synthesized at room temperature for $24 \mathrm{~h}$ following the general procedure using 4-(buta-1,3-dien-2-yl)-1,1'biphenyl $1 \mathrm{a}$ (51.6 mg, $0.25 \mathrm{mmol}, 1.0$ equiv), allylamine $\mathbf{2 p}$ (75 $\mu \mathrm{L}, 1.00 \mathrm{mmol}, 4.0$ equiv), $\mathrm{Ni}(\mathrm{cod})_{2}(3.4 \mathrm{mg}, 0.0125$ mmol, $5 \mathrm{~mol} \%),(R, R)-B_{\text {enzP* }}(4.2 \mathrm{mg}, 0.015 \mathrm{mmol}, 6$ mol\%), 2,2,2-trifluoroethanol ( $19 \mu \mathrm{L}, 0.25 \mathrm{mmol}, 1.0$ equiv) and mesitylene ( $0.50 \mathrm{~mL}, 0.5 \mathrm{M})$. Consumption of 1a: $99 \%$, conversion of 3 ap: $71 \%$, r.r. $=8: 1$. Purification by flash chromatography over silica gel (Pentane:EtOAc $=1: 1$ ) led to the desired product as a colorless viscous oil $(46.1 \mathrm{mg}, 70 \%$ yield, $94 \%$ ee).

${ }^{1} \mathrm{H}$ NMR $\left(400 \mathrm{MHz}, \mathrm{CDCl}_{3}\right) \delta(\mathrm{ppm})=7.65-7.55(\mathrm{~m}, 4 \mathrm{H}, \mathrm{H}-7+\mathrm{H}-10), 7.51-7.41(\mathrm{~m}, 4 \mathrm{H}, \mathrm{H}-$ $6+H-11), 7.39-7.31(\mathrm{~m}, 1 \mathrm{H}, H-12), 6.03-5.88(\mathrm{~m}, 1 \mathrm{H}, H-14), 5.34\left(\mathrm{~d},{ }^{2} J_{H H}=1.1 \mathrm{~Hz}, 1 \mathrm{H}, H-\right.$ 4), $5.29(\mathrm{~s}, 1 \mathrm{H}, \mathrm{H}-4), 5.21\left(\mathrm{dd},{ }^{3} J_{\mathrm{HH}}=17.2,{ }^{2} \mathrm{~J}_{\mathrm{HH}}=1.6 \mathrm{~Hz}, 1 \mathrm{H}, \mathrm{H}-15\right), 5.11\left(\mathrm{dd},{ }^{3} J_{\mathrm{HH}}=10.4\right.$, $\left.{ }^{2} J_{\mathrm{HH}}=1.2 \mathrm{~Hz}, 1 \mathrm{H}, H-15\right), 3.78\left(\mathrm{q},{ }^{3} J_{\mathrm{HH}}=6.5 \mathrm{~Hz}, 1 \mathrm{H}, \mathrm{H}-2\right), 3.36\left(\mathrm{dd},{ }^{2} J_{\mathrm{HH}}=14.0,{ }^{3} J_{\mathrm{HH}}=5.8\right.$ $\mathrm{Hz}, 1 \mathrm{H}, H-13), 3.28\left(\mathrm{dd},{ }^{2} J_{\mathrm{HH}}=14.0,{ }^{3} \mathrm{~J}_{\mathrm{HH}}=6.2 \mathrm{~Hz}, 1 \mathrm{H}, H-13\right), 1.36(\mathrm{~s}, 1 \mathrm{H}, \mathrm{NH}), 1.25\left(\mathrm{~d},{ }^{3} \mathrm{~J}_{\mathrm{HH}}\right.$ $=6.6 \mathrm{~Hz}, 3 \mathrm{H}, \mathrm{H}-1)$.

${ }^{13} \mathrm{C}\left\{{ }^{1} \mathrm{H}\right\}$ NMR $\left(100 \mathrm{MHz}, \mathrm{CDCl}_{3}\right) \delta(\mathrm{ppm})=151.7(C-3), 140.9(C-9), 140.3(C-5+C-8), 137.3$ (CH-14), $128.9(\mathrm{CH}-11), 127.5(\mathrm{CH}-6), 127.4(\mathrm{CH}-12), 127.13(\mathrm{CH}-10), 127.07(\mathrm{CH}-7), 115.9$ $\left(\mathrm{CH}_{2}-15\right), 112.7\left(\mathrm{CH}_{2}-4\right), 56.7(\mathrm{CH}-2), 50.1\left(\mathrm{CH}_{2}-13\right), 21.8\left(\mathrm{CH}_{3}-1\right)$.

HRMS (ESI+): calculated [M+H] $]^{+}$for $\mathrm{C}_{19} \mathrm{H}_{22} \mathrm{~N}^{+}: 264.1747$; found: 264.1752.

IR (neat) $v\left(\mathrm{~cm}^{-1}\right): 3077,3030,2970,2925,2868,1642,1601,1486,1447,1370,1120,1078$, 1006, 995, 910, 843, 769, 739, 695.

HPLC: $94 \%$ ee, chiral stationary column: $\mathrm{OJ}-\mathrm{H}$, mobile phase: hexane/ $\mathrm{PrOH}=97 / 3,1.0$ $\mathrm{mL} / \mathrm{min}, 254 \mathrm{~nm}, 30^{\circ} \mathrm{C}, \mathrm{t}_{\mathrm{R}}($ minor $)=8.7 \mathrm{~min}, \mathrm{t}_{\mathrm{R}}$ (major) $=10.7 \mathrm{~min}$.

$[\alpha]^{20} \mathrm{D}=-12.6\left(c 1.0, \mathrm{CHCl}_{3}\right)$. 
<Chromatogram>

mAU

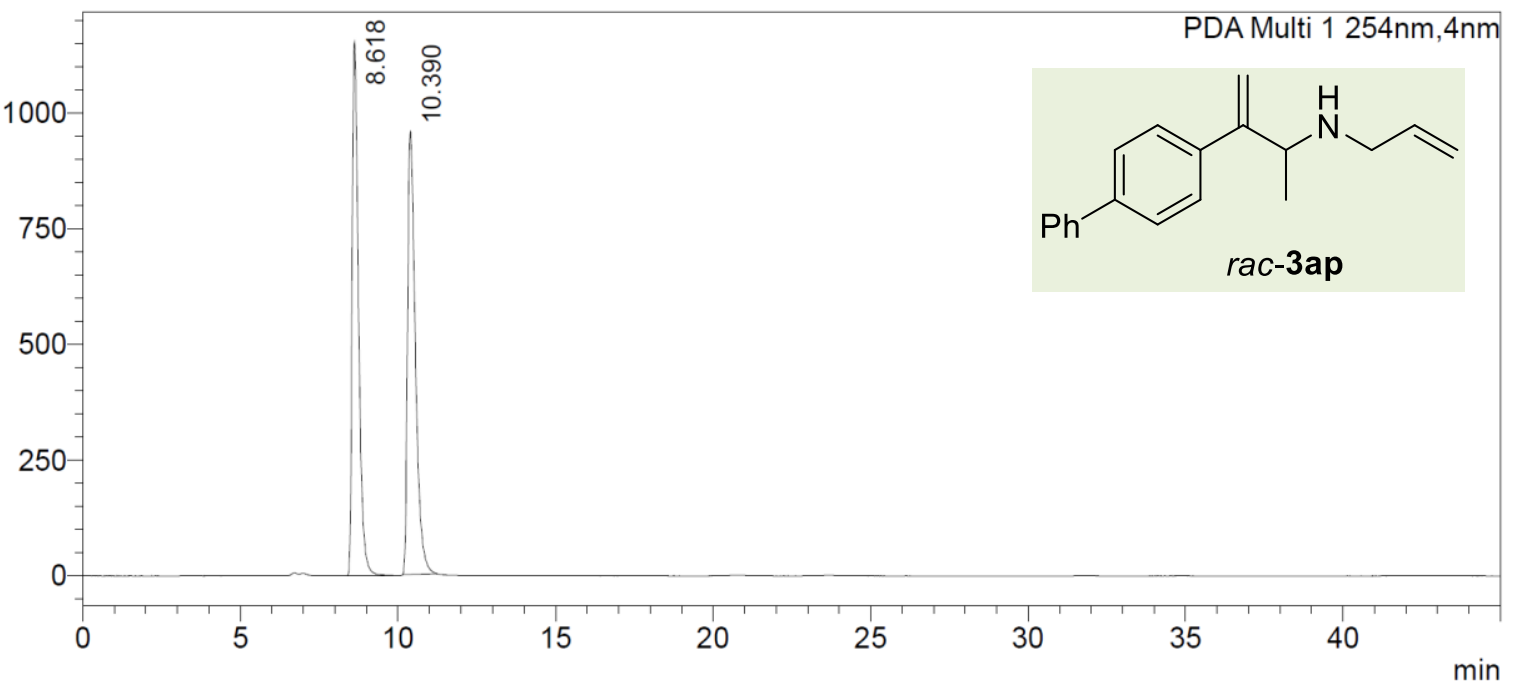

<Peak Table>

PDA Ch1 254nm

\begin{tabular}{|l|l} 
Peak\# Ret. Time & Area\%
\end{tabular}

\begin{tabular}{|r|r|r|}
\hline 1 & 8.618 & 49.708 \\
\hline 2 & 10.390 & 50.292 \\
\hline Total & & 100.000 \\
\hline
\end{tabular}

<Chromatogram>

$\mathrm{mAU}$

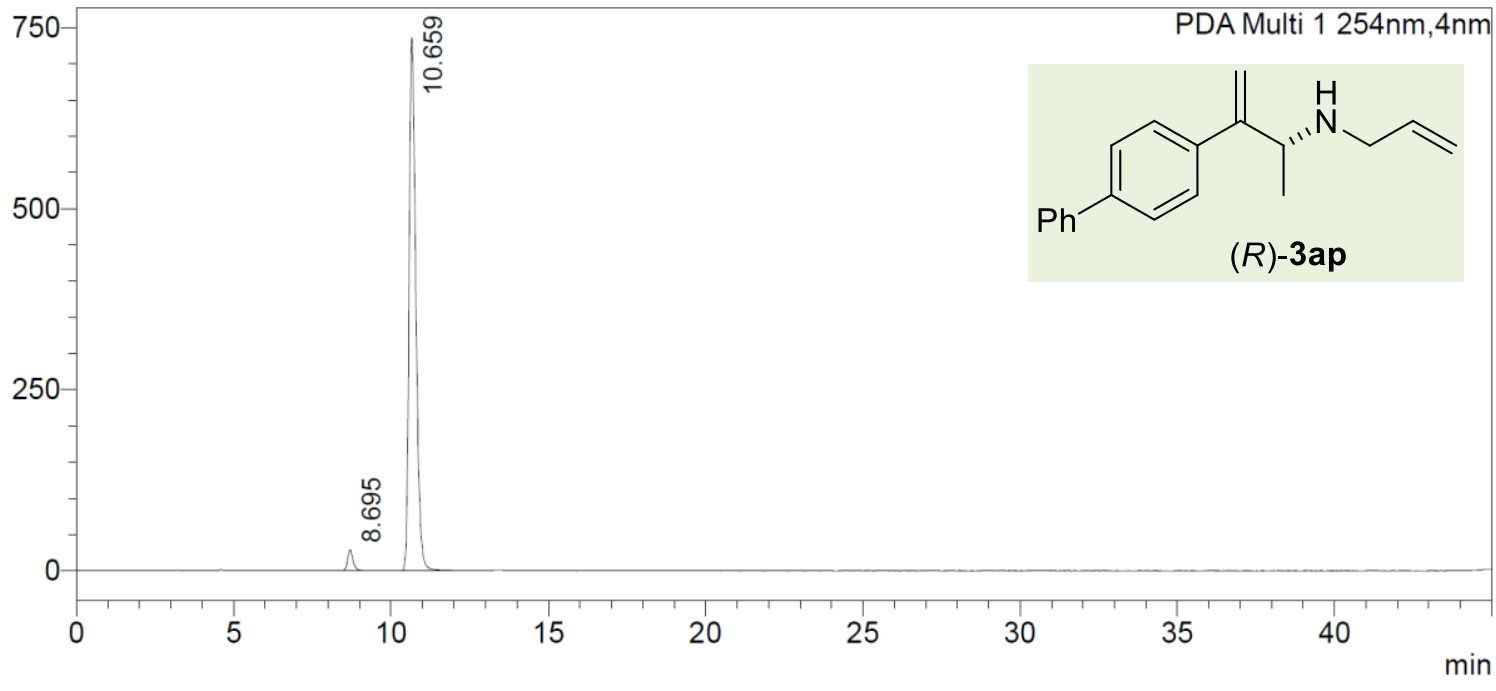

<Peak Table>

PDA Ch1 254nm

Peak\# Ret. Time $\quad$ Area\%

\begin{tabular}{|r|r|r|}
\hline 1 & 8.695 & 3.033 \\
\hline 2 & 10.659 & 96.967 \\
\hline Total & & 100.000 \\
\hline
\end{tabular}




\section{Rh-catalyzed deallylation of 3ap}<smiles>C=CCNC(C(=C)c1ccc(-c2ccccc2)cc1)C(C)C</smiles>

$(R)$-3ap
$\underset{\mathrm{Mh}\left(\mathrm{PPh}_{3}\right)_{3} \mathrm{Cl}(1 \mathrm{~mol} \%)}{\stackrel{\mathrm{MeCN} / \mathrm{H}_{2} \mathrm{O}, \text { reflux }}{\longrightarrow}}$

$\mathrm{Ph}^{\prime}$<smiles>C=C(c1ccc(-c2ccccc2)cc1)[C@H](C)N</smiles>

$(R)-6 \mathbf{a}$

Modification of a reported method, ${ }^{2} \mathrm{Rh}\left(\mathrm{PPh}_{3}\right)_{3} \mathrm{Cl}(1.8 \mathrm{mg}, 0.002 \mathrm{mmol}, 1 \% \mathrm{~mol})$ was added to a solution of $(R)$-3ap (52.7 mg, $0.20 \mathrm{mmol}, 94 \%$ ee, 1.0 equiv) in $\mathrm{MeCN} / \mathrm{H}_{2} \mathrm{O}$ (degassed solvent, $2.0 \mathrm{~mL}, \mathrm{~V} / \mathrm{V}=85 / 15)$ under nitrogen atmosphere, and the mixture was heated at reflux $\left(90^{\circ} \mathrm{C}\right)$ for $7 \mathrm{~h}$. The reaction mixture was cooled to room temperature, filtered over a short pad of silica gel (EtOAc and $\mathrm{CH}_{2} \mathrm{Cl}_{2}$ washing, with $5 \% \mathrm{MeOH}$ ) and concentrated in vacuo to afford the crude mixture. Consumption of 3ap: $97 \%$, conversion of $6 \mathbf{a}: 81 \%$. Then the residue was purified by silica gel column chromatography $\left(\mathrm{CH}_{2} \mathrm{Cl}_{2}: \mathrm{MeOH}=10: 1\right)$ afforded 6 a (36.3 mg, $81 \%$ yield, $94 \%$ ee) as a light yellow solid.

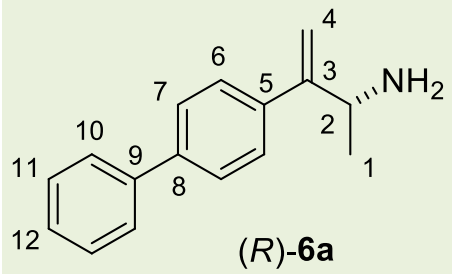

$141.9(C-8), 141.8(C-9), 140.8(C-5), 129.9(\mathrm{CH}-11), 128.5(\mathrm{CH}-12), 128.2(\mathrm{CH}-6), 128.1$ (CH-7), $127.9(\mathrm{CH}-10), 111.6\left(\mathrm{CH}_{2}-4\right), 50.6(\mathrm{CH}-2), 22.3\left(\mathrm{CH}_{3}-1\right)$.

m.p. $=206.0-208.5^{\circ} \mathrm{C}$.

HRMS $\left(\mathrm{ESI}^{+}\right)$: calculated $[\mathrm{M}+\mathrm{H}]^{+}$for $\mathrm{C}_{16} \mathrm{H}_{18} \mathrm{NO}^{+}:$224.1434; found: 224.1442 .

IR (neat) $v\left(\mathrm{~cm}^{-1}\right): 3034,2975,2867,2806,2709,2644,2497,1620,1540,1513,1487,1449$, 1377, 1261, 1207, 1145, 1116, 1077, 1004, 909, 840, 769, 737, 694.

HPLC: $94 \%$ ee, chiral stationary column: AD-H, mobile phase: hexane/ $\mathrm{PrOH}=95 / 5,1.0$ $\mathrm{mL} / \mathrm{min}, 254 \mathrm{~nm}, 30^{\circ} \mathrm{C}, \mathrm{t}_{\mathrm{R}}$ (minor) $=9.9 \mathrm{~min}, \mathrm{t}_{\mathrm{R}}$ (major) $=11.6 \mathrm{~min}$.

$[\alpha]^{20} \mathrm{D}=-60.6(c 0.05, \mathrm{MeOH})$. 
$<$ Chromatogram>

mAU

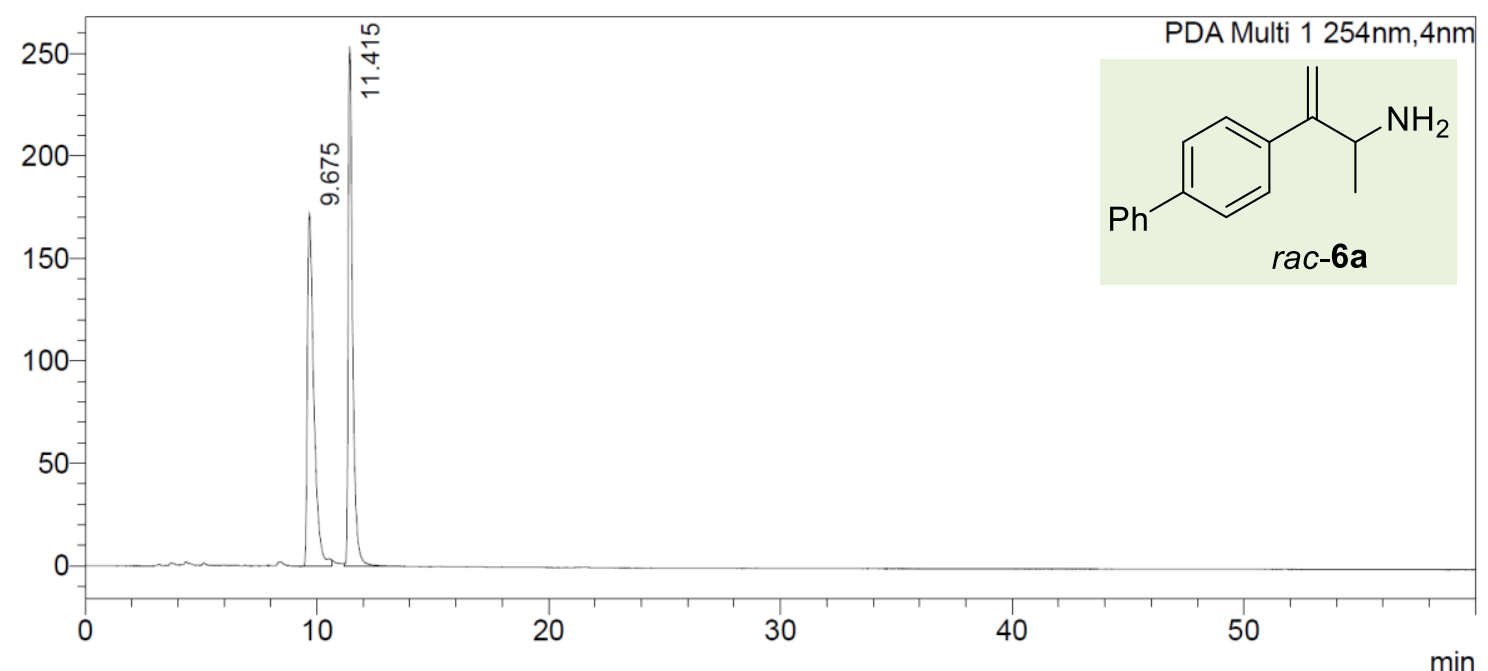

<Peak Table>

PDA Ch1 254nm

Peak\# Ret. Time $\quad$ Area \%

\begin{tabular}{|r|r|r|}
\hline 1 & 9.675 & 50.048 \\
\hline 2 & 11.415 & 49.952 \\
\hline Total & & 100.000 \\
\hline
\end{tabular}

\section{$<$ Chromatogram $>$}

mAU

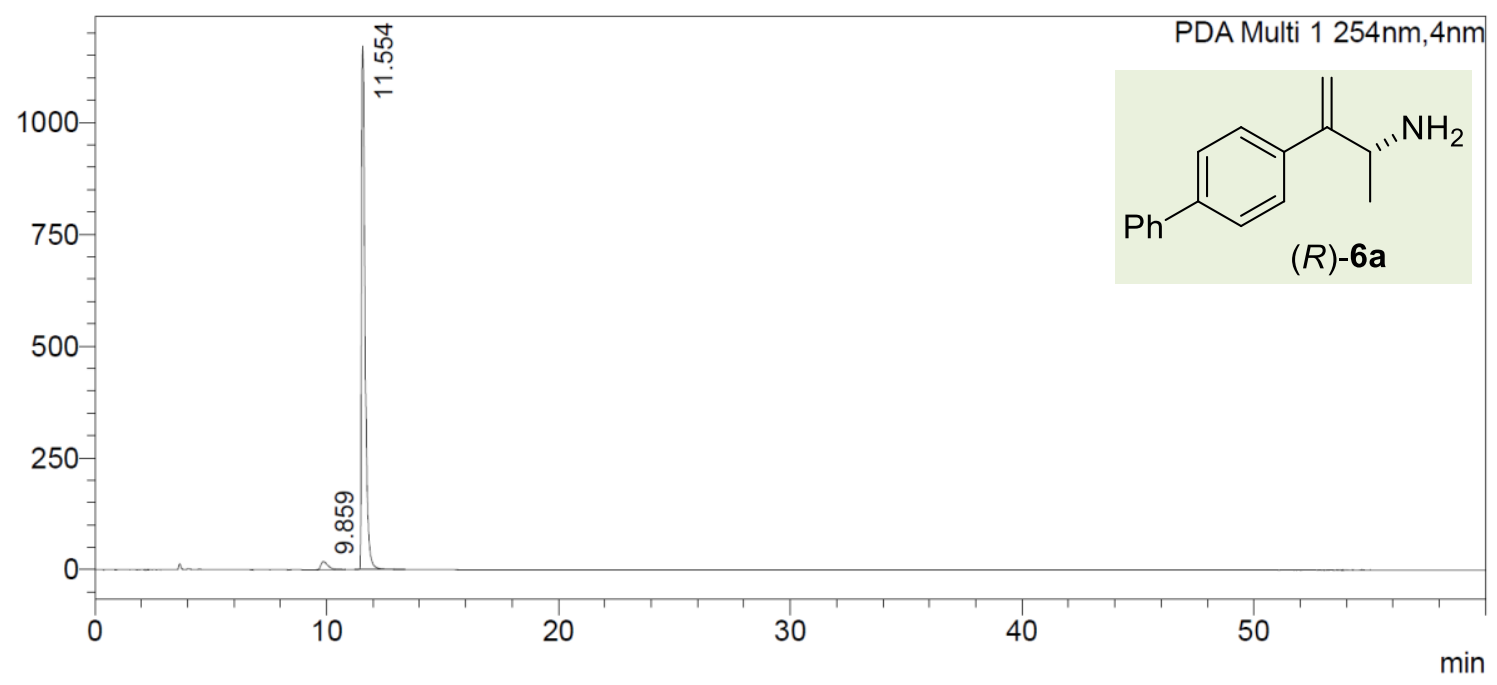

\section{<Peak Table>}

PDA Ch1 254nm

\begin{tabular}{|l|l|}
\hline Peak\# Ret. Time & Area\% \\
\hline
\end{tabular}

\begin{tabular}{|r|r|r|}
\hline 1 & 9.859 & 3.052 \\
\hline 2 & 11.554 & 96.948 \\
\hline Total & & 100.000 \\
\hline
\end{tabular}




\section{Improved sets of reaction conditions}

\subsection{Reduced Catalyst Loading}

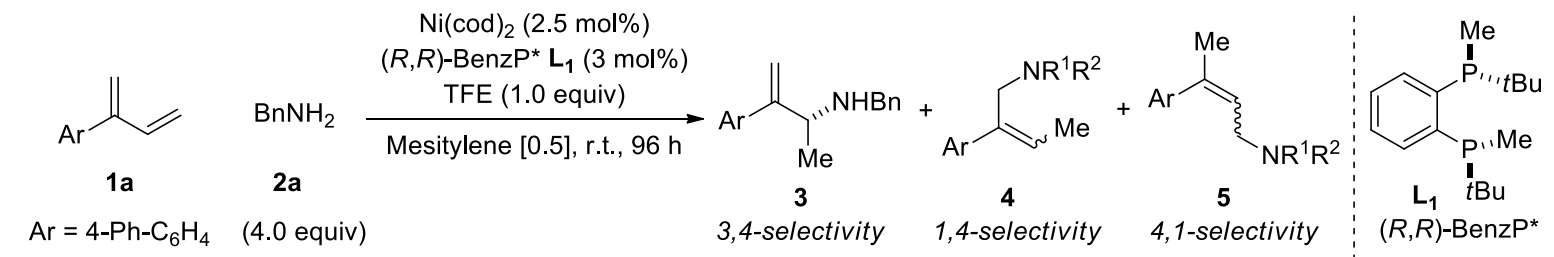

Performed at room temperature for $96 \mathrm{~h}$ following the general procedure using 4-(buta-1,3dien-2-yl)-1,1'-biphenyl 1a (103 mg, $0.50 \mathrm{mmol}, 1.0$ equiv), benzylamine 2a (219 $\mu \mathrm{L}, 2.0$ mmol, 4.0 equiv), $\mathrm{Ni}(\mathrm{cod}))_{2}$ (3.4 mg, $\left.0.0125 \mathrm{mmol}, 2.5 \mathrm{~mol} \%\right),(R, R)-\mathrm{BenzP}^{*}$ (4.2 mg, 0.015 mmol, $3.0 \mathrm{~mol} \%$ ), 2,2,2-trifluoroethanol (38 $\mu \mathrm{L}, 0.50 \mathrm{mmol}, 1.0$ equiv) and mesitylene $(1.0 \mathrm{~mL}, 0.50 \mathrm{M})$. Consumption of $1 \mathbf{a}:>99 \%$, conversion of 3aa: $84 \%$, r.r. $=6: 1$. Purification by flash chromatography over silica gel (pentane:EtOAc $=5: 1$ ) led to the desired product as a yellow solid (120.8 $\mathrm{mg}, 77 \%$ yield, 92\% ee).

\subsection{Reduced Amine Loading}

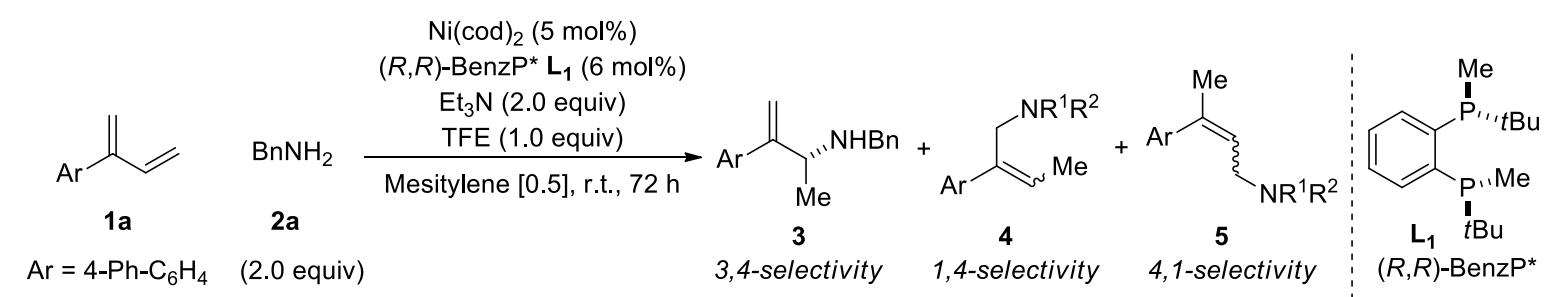

In a $\mathrm{N}_{2}$-filled glovebox, $\mathrm{Ni}(\mathrm{cod})_{2}(3.4 \mathrm{mg}, 0.0125 \mathrm{mmol}, 5 \mathrm{~mol} \%)$ and $(R, R)$-BenzP* $\mathbf{L}_{1}(4.2$ $\mathrm{mg}, 0.015 \mathrm{mmol}, 6 \mathrm{~mol} \%$ ) were charged in a $5 \mathrm{~mL}$ Schlenk tube and dissolved in anhydrous mesitylene $(0.50 \mathrm{~mL}, 0.50 \mathrm{M})$. After stirring at room temperature for $10 \mathrm{~min}$, the diene 1a (51.6 mg, 0.25 mmol, 1.0 equiv), $\mathrm{BnNH}_{2} 2 \mathrm{a}$ (55 $\mu \mathrm{L}, 0.50$ mmol, 4.0 equiv), $\mathrm{Et}_{3} \mathrm{~N}$ (28 $\mu \mathrm{L}, 0.50$ mmol, 4.0 equiv) and TFE (19 $\mu \mathrm{L}, 0.25 \mathrm{mmol}, 1.0$ equiv) were added sequentially. The tube was sealed, taken out of the glovebox and the reaction mixture was stirred at room temperature for $72 \mathrm{~h}$. The reaction mixture was filtered over a short pad of silica gel, washed with ethyl acetate $(10 \mathrm{~mL})$ and concentrated under vacuum to afford the crude mixture. The conversion and isomeric ratio were determined by ${ }^{1} \mathrm{H}$ NMR analysis of the crude reaction mixture using $\mathrm{CH}_{2} \mathrm{Br}_{2}$ as an internal standard. Consumption of 1a: $85 \%$, conversion of 3ap: $75 \%$, r.r. $=8: 1$. Purification by flash chromatography over silica gel (Pentane $:$ EtOAc $=5: 1$ ) led to the desired product as a colorless oil $(56.0 \mathrm{mg}, 72 \%$ yield, $92 \%$ ee). 


\section{Supporting Organometallics}

\subsection{NMR Monitoring}

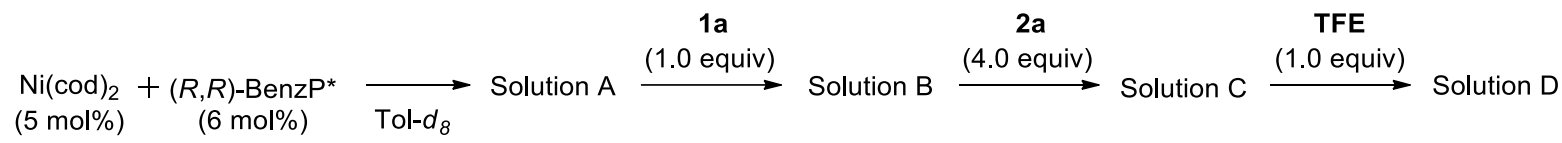

In a $\mathrm{N}_{2}$-filled glovebox, a stock solution of $\mathrm{Ni}(\operatorname{cod})_{2}(6.9 \mathrm{mg}, 0.025 \mathrm{mmol})$ and $(\mathrm{R}, \mathrm{R}) \mathrm{BenzP}$ * $(8.5 \mathrm{mg}, 0.030 \mathrm{mmol})$ in toluene- $d_{8}(1.0 \mathrm{~mL})$ was stirred for $5 \mathrm{~min}$, and $0.40 \mathrm{~mL}$ of the resulting pale orange solution was introduced in a J-Young NMR tube (solution A). ${ }^{1} \mathrm{H}$ and ${ }^{31} \mathrm{P}\left\{{ }^{1} \mathrm{H}\right\}$ NMR of this solution were immediately recorded. In another J-Young tube was weighted 4-(buta-1,3-dien-2-yl)-1,1'-biphenyl 1a (41.3 mg, $0.20 \mathrm{mmol}, 1.0$ equiv), to which was added $0.40 \mathrm{~mL}$ of the $\mathrm{Ni}(\operatorname{cod})_{2} /(\mathrm{R}, \mathrm{R})$ BenzP* $\mathrm{P}^{*}$ stock solution $\mathrm{A}$, which resulted in an immediate change of color from pale orange to dark orange (solution $B$ ). ${ }^{1} \mathrm{H}$ and ${ }^{31} \mathrm{P}\left\{{ }^{1} \mathrm{H}\right\} \mathrm{NMR}$ of this solution were immediately recorded. The $\mathrm{J}$-Young tube containing solution $\mathrm{B}$ was brought back into the glovebox, and neat $\mathrm{BnNH}_{2} 2 \mathrm{a}(86.0 \mathrm{mg}, 0.80 \mathrm{mmol}, 4.0$ equiv) was added to the tube (solution $\mathrm{C}$ ). ${ }^{1} \mathrm{H}$ and ${ }^{31} \mathrm{P}\left\{{ }^{1} \mathrm{H}\right\} \mathrm{NMR}$ of this solution were immediately recorded. The J-Young tube containing solution $\mathrm{C}$ was brought back into the glove-box, and neat 2,2,2-trifluoroethanol (20.0 $\mathrm{mg}, 0.20 \mathrm{mmol}, 1.0$ equiv) was added to the tube, which resulted in a slight change of color from dark orange to pale orange (solution $\mathrm{D}) .{ }^{1} \mathrm{H},{ }^{19} \mathrm{~F}\left\{{ }^{1} \mathrm{H}\right\}$ and ${ }^{31} \mathrm{P}\left\{{ }^{1} \mathrm{H}\right\}$ NMR of this solution were immediately recorded, and the evolution of the reaction mixture was monitored over $56 \mathrm{~h}$. 


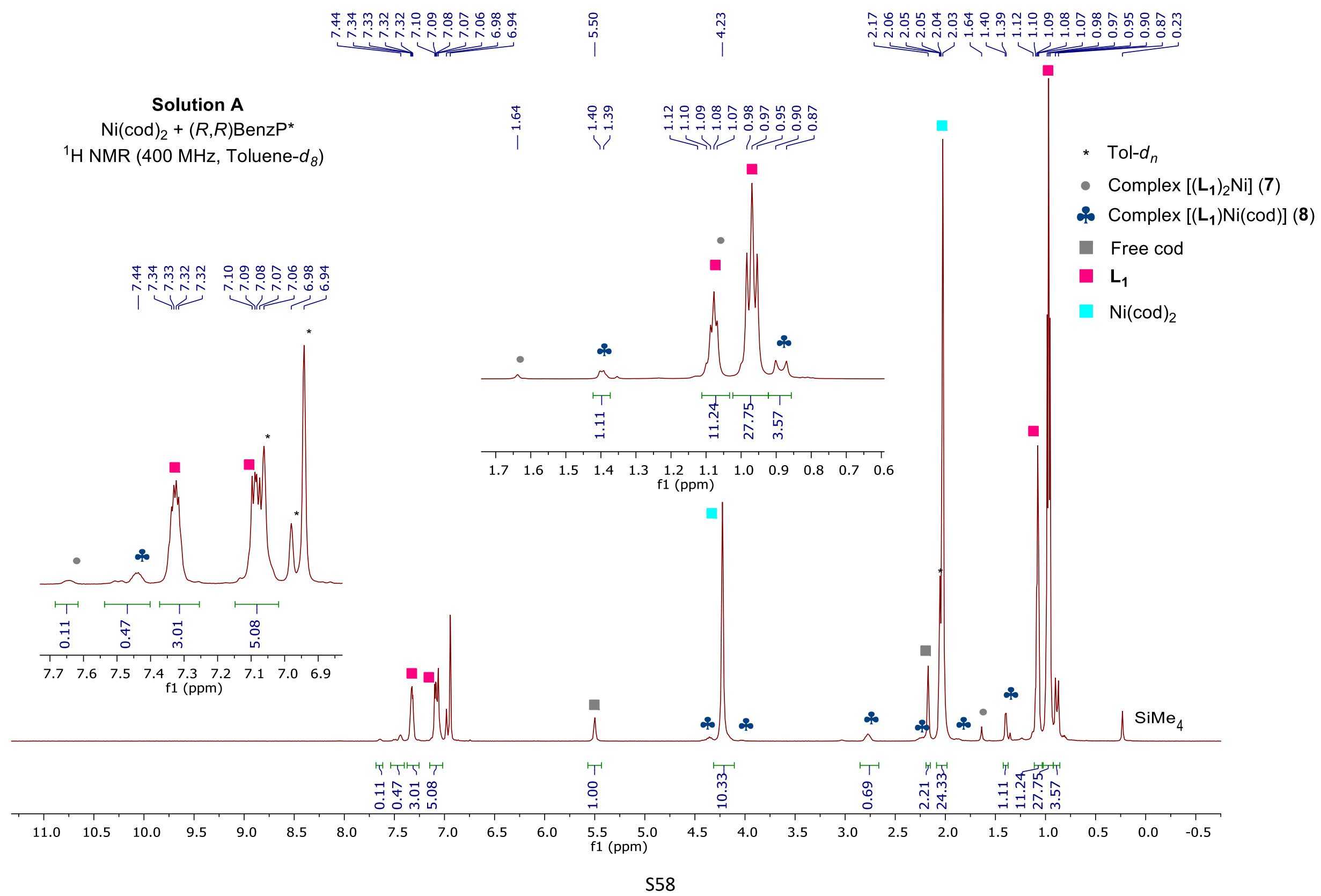




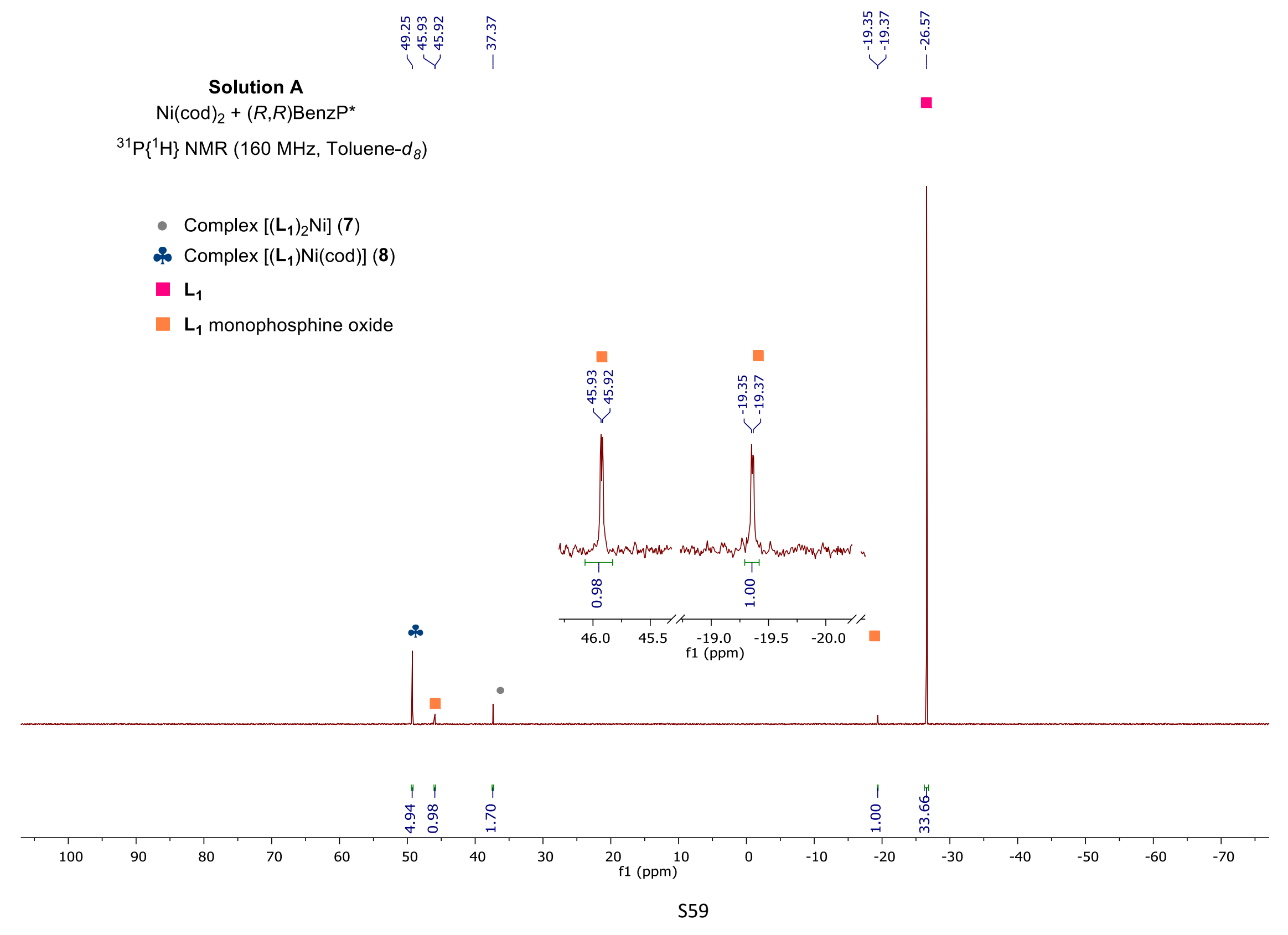




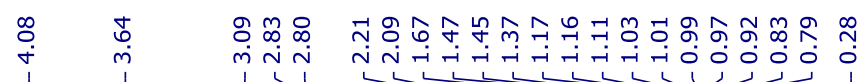

\section{Solution B}

$\mathrm{Ni}(\operatorname{cod})_{2}+(R, R)$ BenzP $^{*}+1 \mathbf{a}$

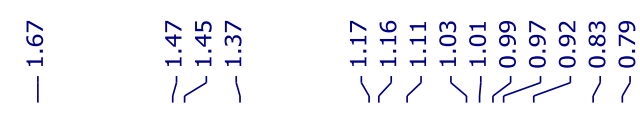

${ }^{1} \mathrm{H}$ NMR (400 MHz, Toluene- $d_{8}$ )

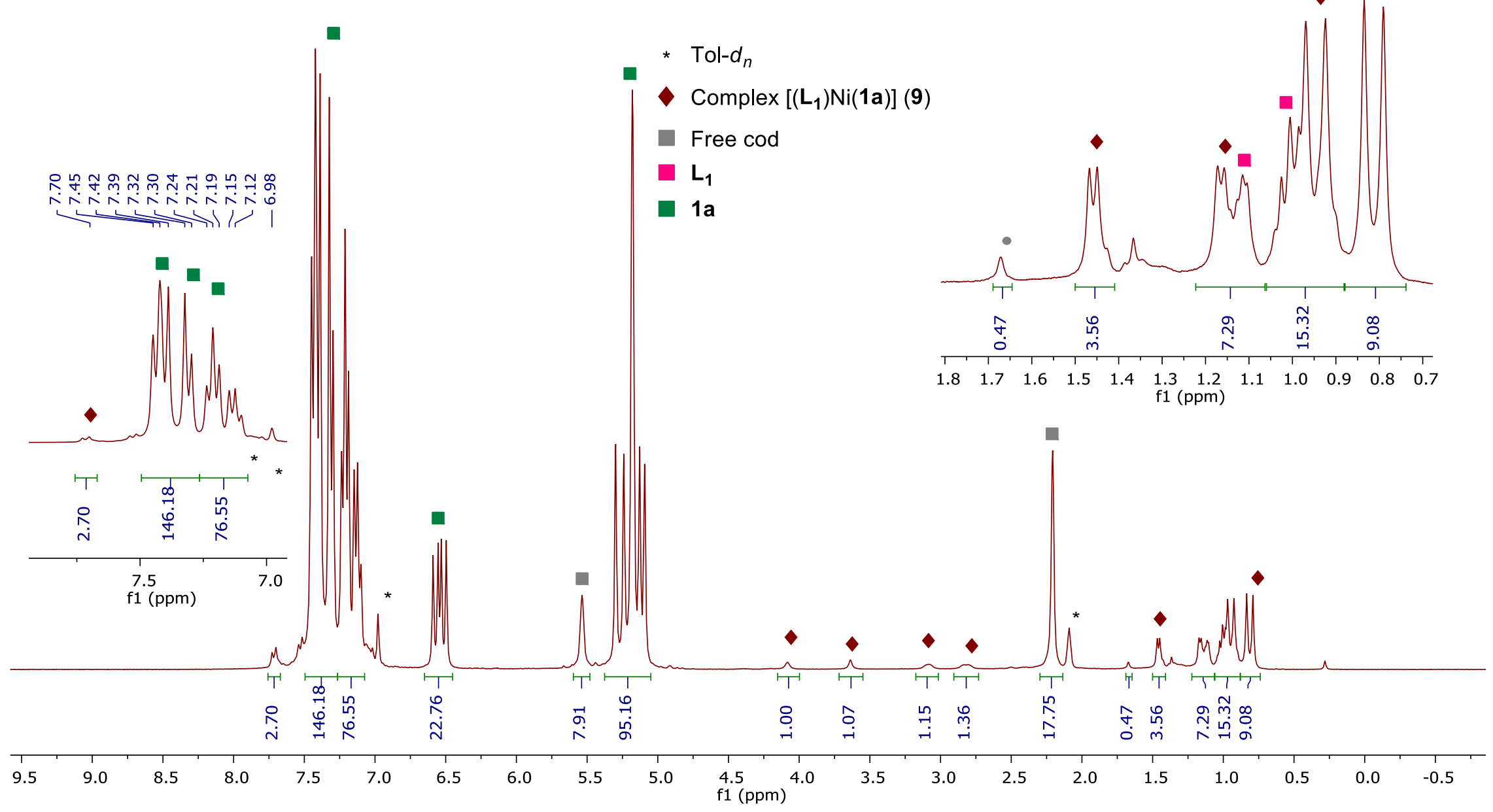




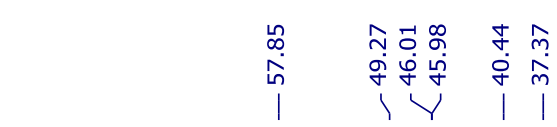

Solution B

$\mathrm{Ni}(\operatorname{cod})_{2}+(R, R)$ BenzP $^{*}+1 \mathrm{a}$

${ }^{31} \mathrm{P}\left\{{ }^{1} \mathrm{H}\right\}$ NMR $\left(160 \mathrm{MHz}\right.$, Toluene- $\left.d_{8}\right)$
- Complex $\left[\left(\mathrm{L}_{1}\right)_{2} \mathrm{Ni}\right](7)$

\& Complex $\left[\left(\mathrm{L}_{1}\right) \mathrm{Ni}(\mathrm{cod})\right](8)$

$\checkmark$ Complex $\left[\left(\mathrm{L}_{1}\right) \mathrm{Ni}(\mathbf{1 a})\right](\mathbf{9})$

- $\mathrm{L}_{1}$ monophosphine oxide

- $\mathrm{L}_{1}$
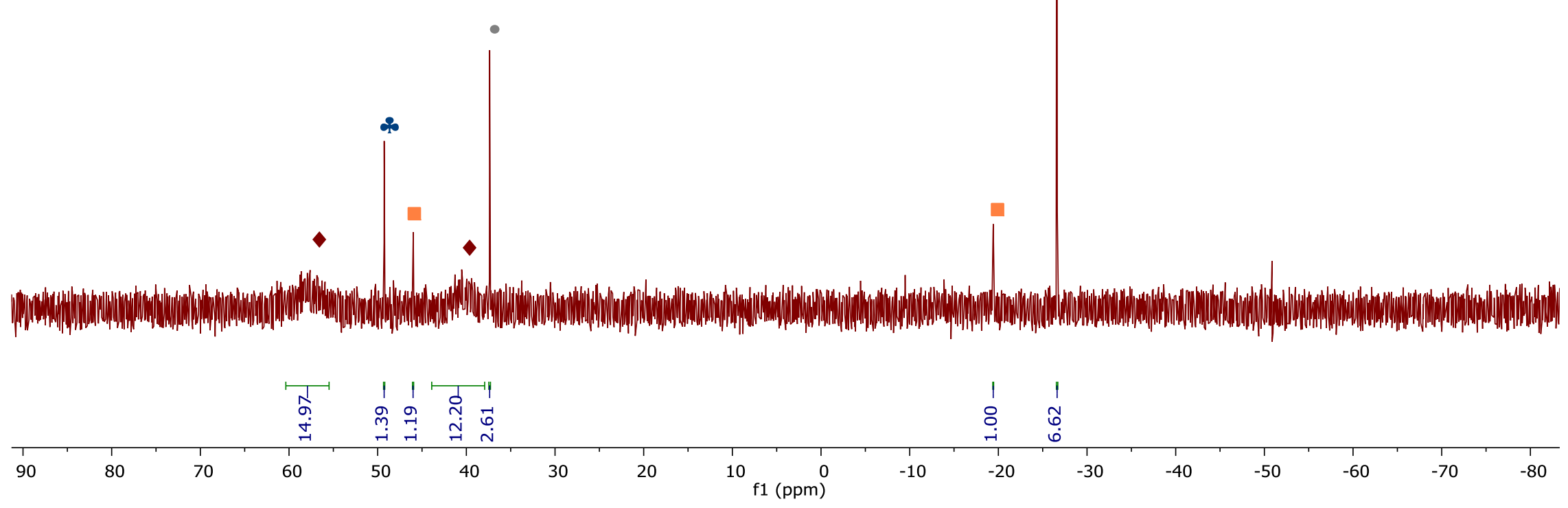


\begin{tabular}{|c|c|c|c|}
\hline 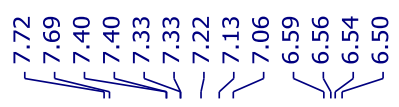 & 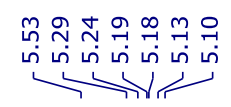 & 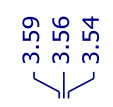 & 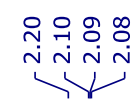 \\
\hline
\end{tabular}

Solution $\mathbf{C}$

$\mathrm{Ni}(\operatorname{cod})_{2}+(R, R)$ Benz $^{*}+1 \mathbf{a}+2 \mathbf{a}$

${ }^{1} \mathrm{H}$ NMR (400 MHz, Toluene- $d_{8}$ )

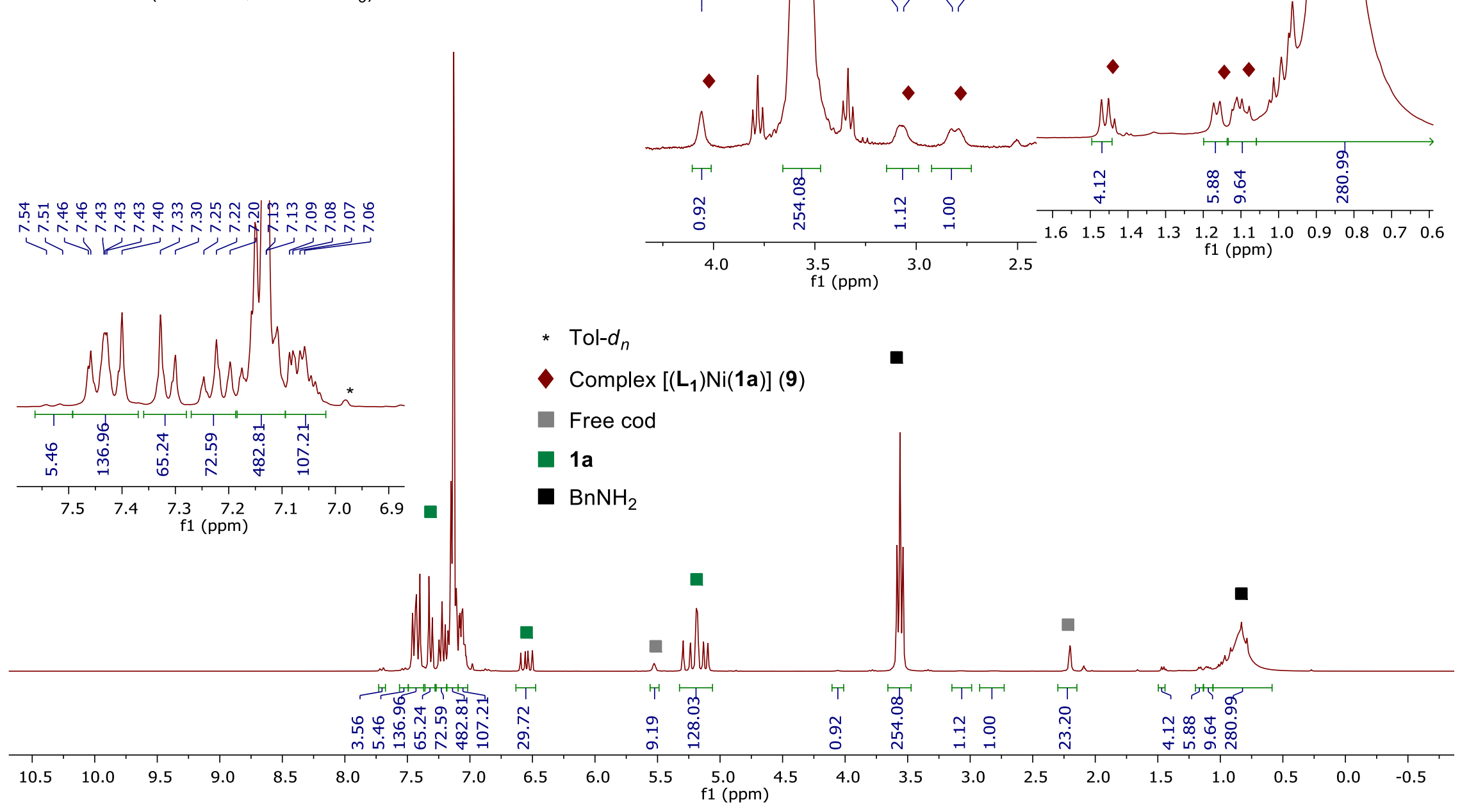




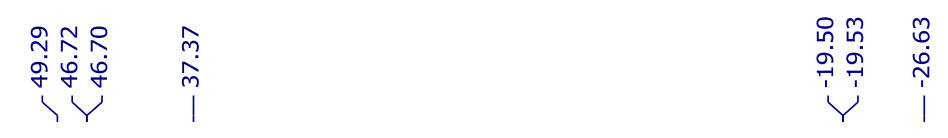

Solution C

$\mathrm{Ni}(\operatorname{cod})_{2}+(R, R)$ BenzP $^{*}+1 \mathbf{a}+\mathbf{2 a}$

${ }^{31} \mathrm{P}\left\{{ }^{1} \mathrm{H}\right\}$ NMR (160 MHz, Toluene- $\left.d_{8}\right)$
- Complex $\left[\left(\mathrm{L}_{1}\right)_{2} \mathrm{Ni}\right](7)$

\& Complex $\left[\left(\mathrm{L}_{1}\right) \mathrm{Ni}(\operatorname{cod})\right](8)$

- Complex $\left[\left(\mathrm{L}_{1}\right) \mathrm{Ni}(\mathbf{1 a})\right](9)$

- $\mathrm{L}_{1}$ monophosphine oxide

- $\mathrm{L}_{1}$

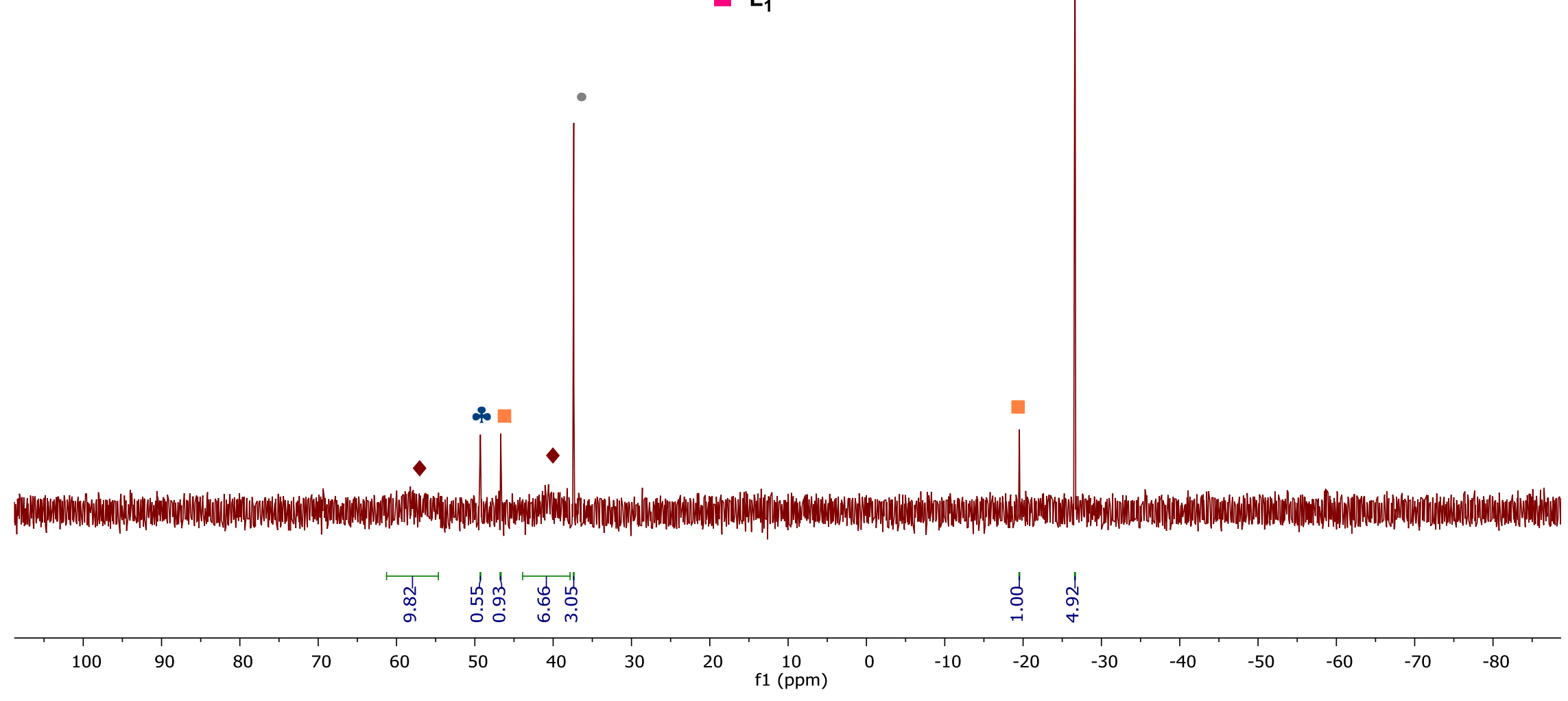




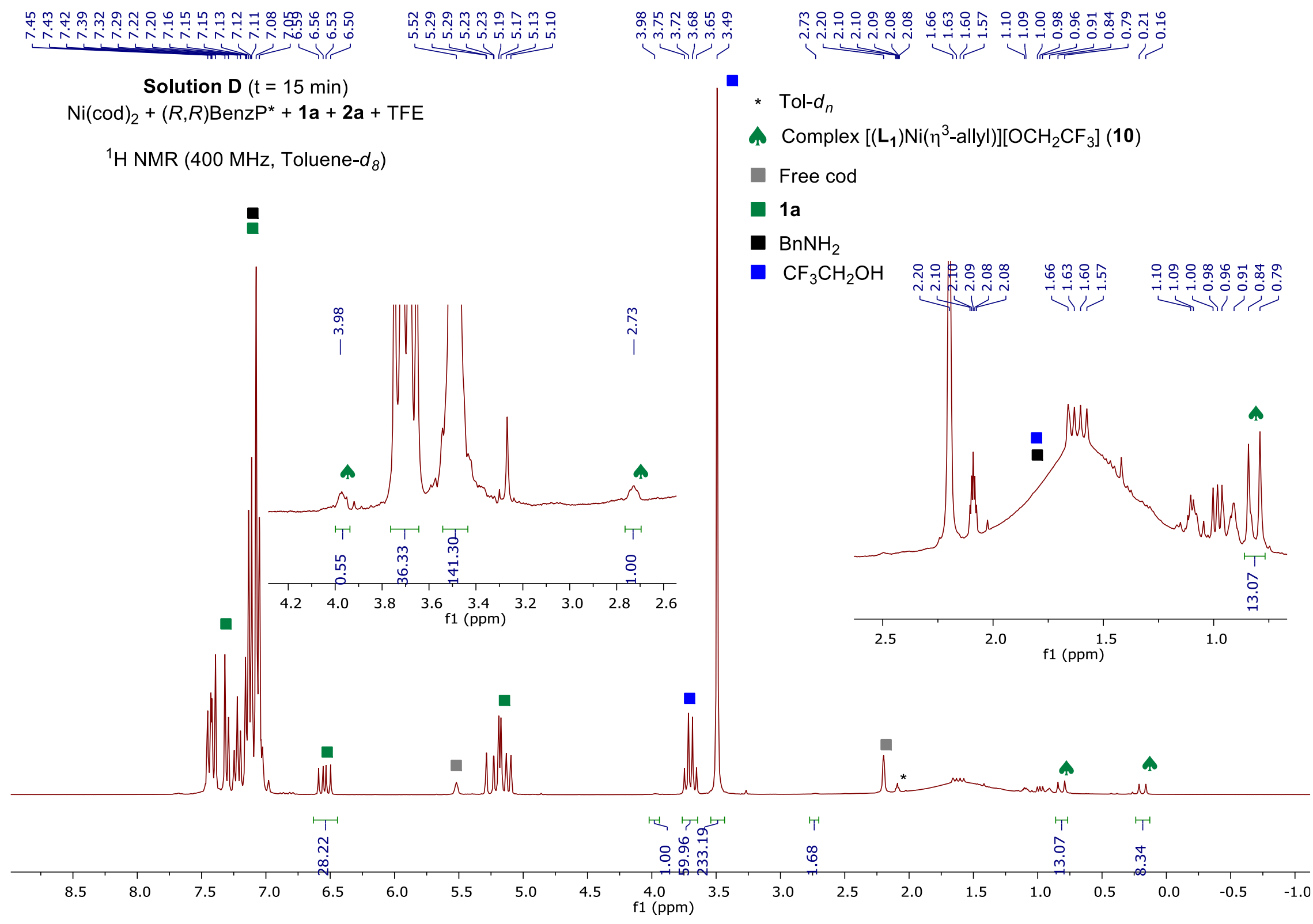




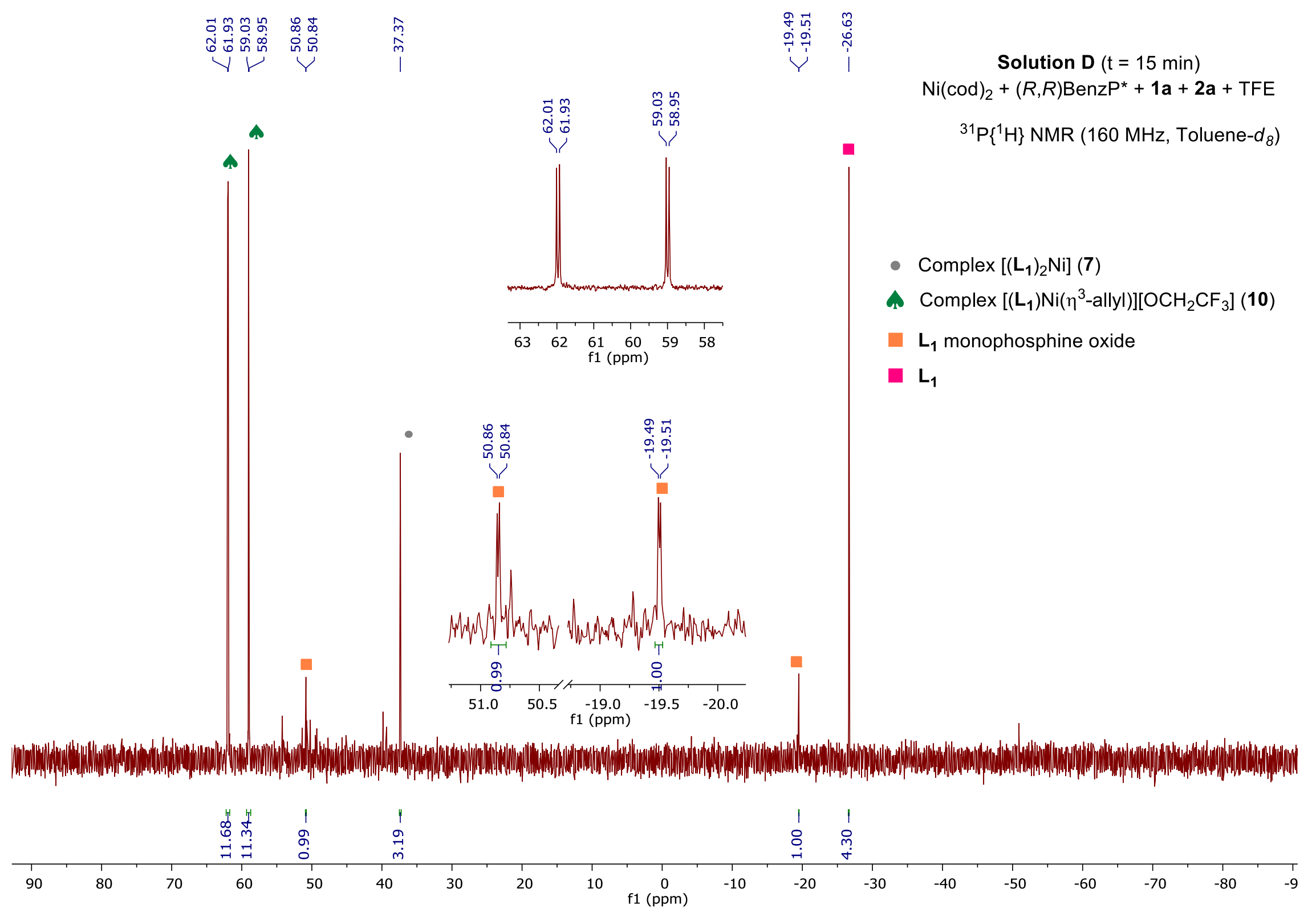


Solution $\mathbf{D}(\mathrm{t}=15 \mathrm{~min})$

$\mathrm{Ni}(\operatorname{cod})_{2}+(R, R)$ BenzP $^{*}+1 \mathbf{a}+\mathbf{2 a}+\mathrm{TFE}$

${ }^{19} \mathrm{~F}\left\{{ }^{1} \mathrm{H}\right\}$ NMR $\left(280 \mathrm{MHz}\right.$, Toluene- $\left.d_{8}\right)$

4. Complex $\left[\left(\mathrm{L}_{1}\right) \mathrm{Ni}\left(\eta^{3}\right.\right.$-allyl $\left.)\right]\left[\mathrm{OCH}_{2} \mathrm{CF}_{3}\right](\mathbf{1 0})$

- $\mathrm{CF}_{3} \mathrm{CH}_{2} \mathrm{OH}$ 
Solution $\mathbf{D}(\mathrm{t}=1 \mathrm{~h})$

$\mathrm{Ni}(\operatorname{cod})_{2}+(R, R)$ BenzP $^{*}+1 \mathrm{a}+\mathbf{2 a}+\mathrm{TFE}$

${ }^{1} \mathrm{H}$ NMR $\left(400 \mathrm{MHz}\right.$, Toluene- $\left.d_{8}\right)$

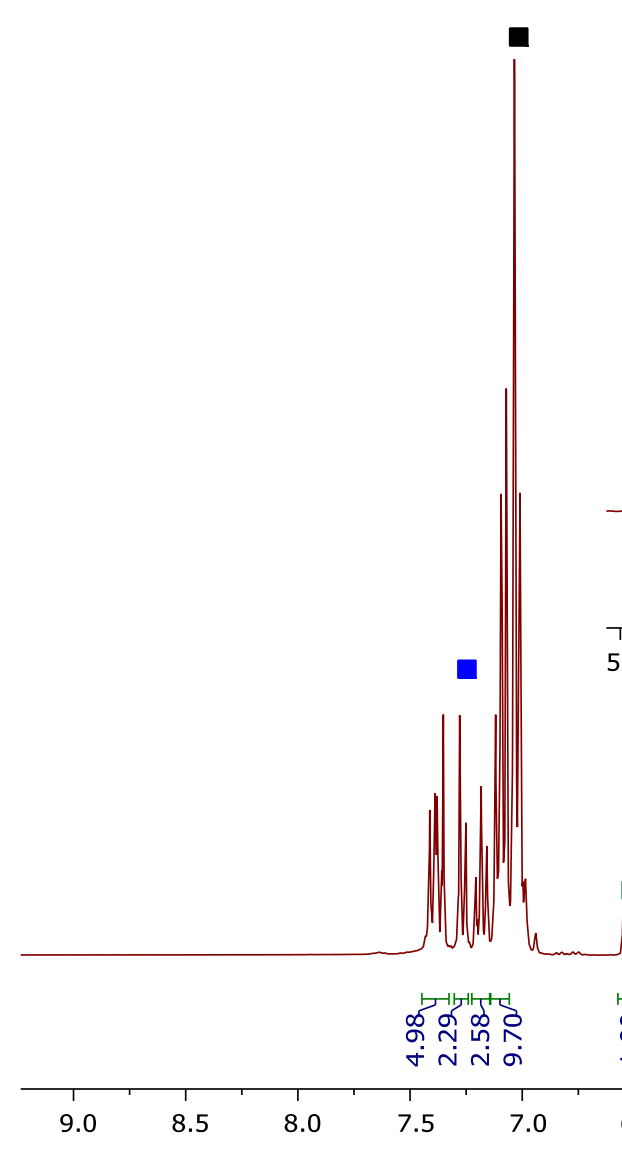

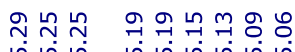

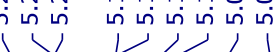

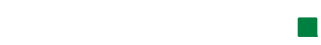

\section{口}

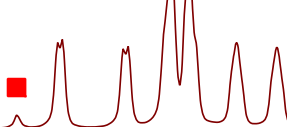

* Tol- $d_{n}$

A. Complex $\left[\left(\mathrm{L}_{1}\right) \mathrm{Ni}\left(\eta^{3}\right.\right.$-allyl $\left.)\right]\left[\mathrm{OCH}_{2} \mathrm{CF}_{3}\right](\mathbf{1 0})$

Free cod

1a

- $\mathrm{BnNH}_{2}$

- $\mathrm{CF}_{3} \mathrm{CH}_{2} \mathrm{OH}$

- 3aa
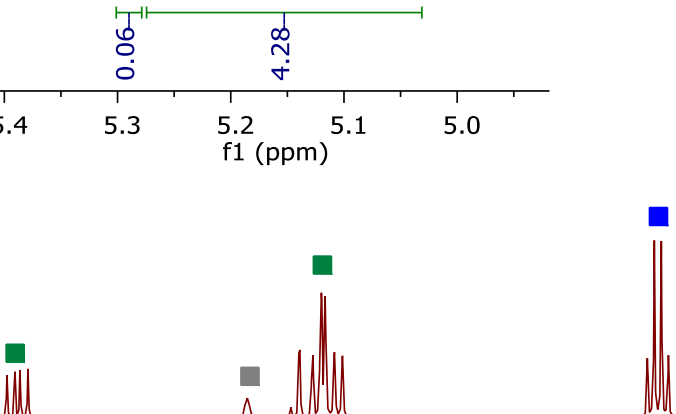

!

(1)

要
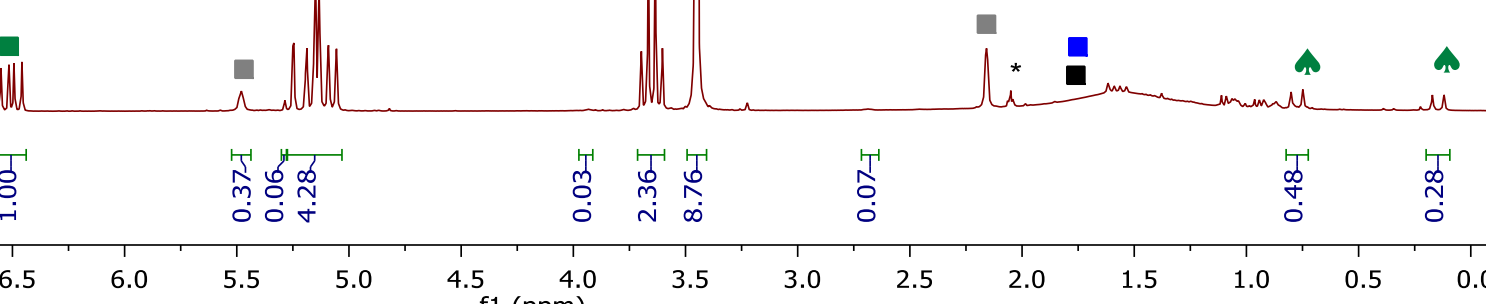


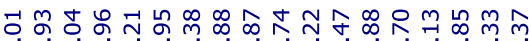 ல่ $\rightarrow$}

\section{Solution $\mathbf{D}(\mathrm{t}=1 \mathrm{~h})$}

$\mathrm{Ni}(\operatorname{cod})_{2}+(R, R)$ BenzP$^{*}+1 \mathrm{a}+2 \mathrm{a}+\mathrm{TFE}$

${ }^{31} \mathrm{P}\left\{{ }^{1} \mathrm{H}\right\}$ NMR $\left(160 \mathrm{MHz}\right.$, Toluene- $\left.d_{8}\right)$

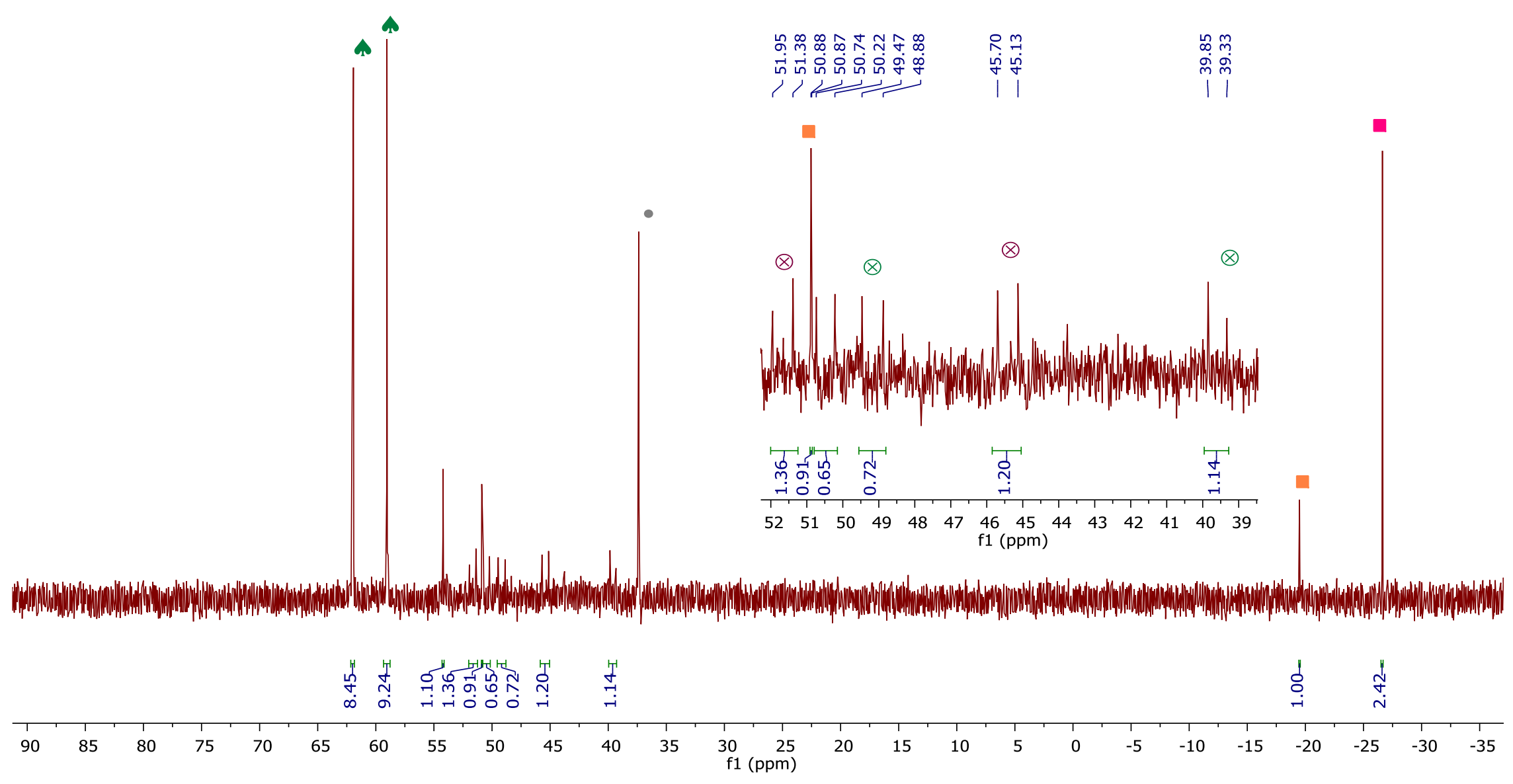

- Complex $\left[\left(\mathrm{L}_{1}\right)_{2} \mathrm{Ni}\right](7)$

A. Complex $\left[\left(\mathrm{L}_{1}\right) \mathrm{Ni}\left(\eta^{3}\right.\right.$-allyl $\left.)\right]\left[\mathrm{OCH}_{2} \mathrm{CF}_{3}\right](10)$

$\otimes$ Complex $\left[\left(\mathrm{L}_{1}\right) \mathrm{Ni}(3 \mathbf{a a})\right]$ (11-major)

$\otimes$ Complex $\left[\left(\mathrm{L}_{1}\right) \mathrm{Ni}(3 \mathbf{a a})\right]$ (11-minor)

$\mathrm{L}_{1}$ monophosphine oxide

- $\mathrm{L}_{1}$ 
Solution $D(\mathrm{t}=1 \mathrm{~h})$

$\mathrm{Ni}(\operatorname{cod})_{2}+(R, R)$ BenzP $^{*}+1 \mathbf{a}+\mathbf{2 a}+\mathrm{TFE}$

${ }^{19} \mathrm{~F}\left\{{ }^{1} \mathrm{H}\right\}$ NMR (280 MHz, Toluene- $\left.d_{8}\right)$

4. Complex $\left[\left(\mathrm{L}_{1}\right) \mathrm{Ni}\left(\eta^{3}\right.\right.$-allyl) $)\left[\mathrm{OCH}_{2} \mathrm{CF}_{3}\right](\mathbf{1 0})$

- $\mathrm{CF}_{3} \mathrm{CH}_{2} \mathrm{OH}$

$\stackrel{\substack{a \\ \infty}}{\infty}$

¿

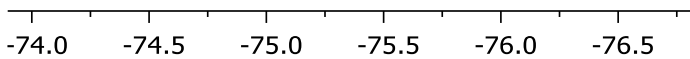

$-77.0$

$-77.5$

$-78.0$

$-78.5-79.0$
f1 (ppm) 
Solution $\mathrm{D}(\mathrm{t}=3$ to $56 \mathrm{~h})$

$\mathrm{Ni}(\operatorname{cod})_{2}+(R, R)$ BenzP $^{*}+\mathbf{1 a}+\mathbf{2 a}+\mathrm{TFE}$

${ }^{1} \mathrm{H}$ NMR $\left(400 \mathrm{MHz}\right.$, Toluene- $\left.d_{8}\right)$

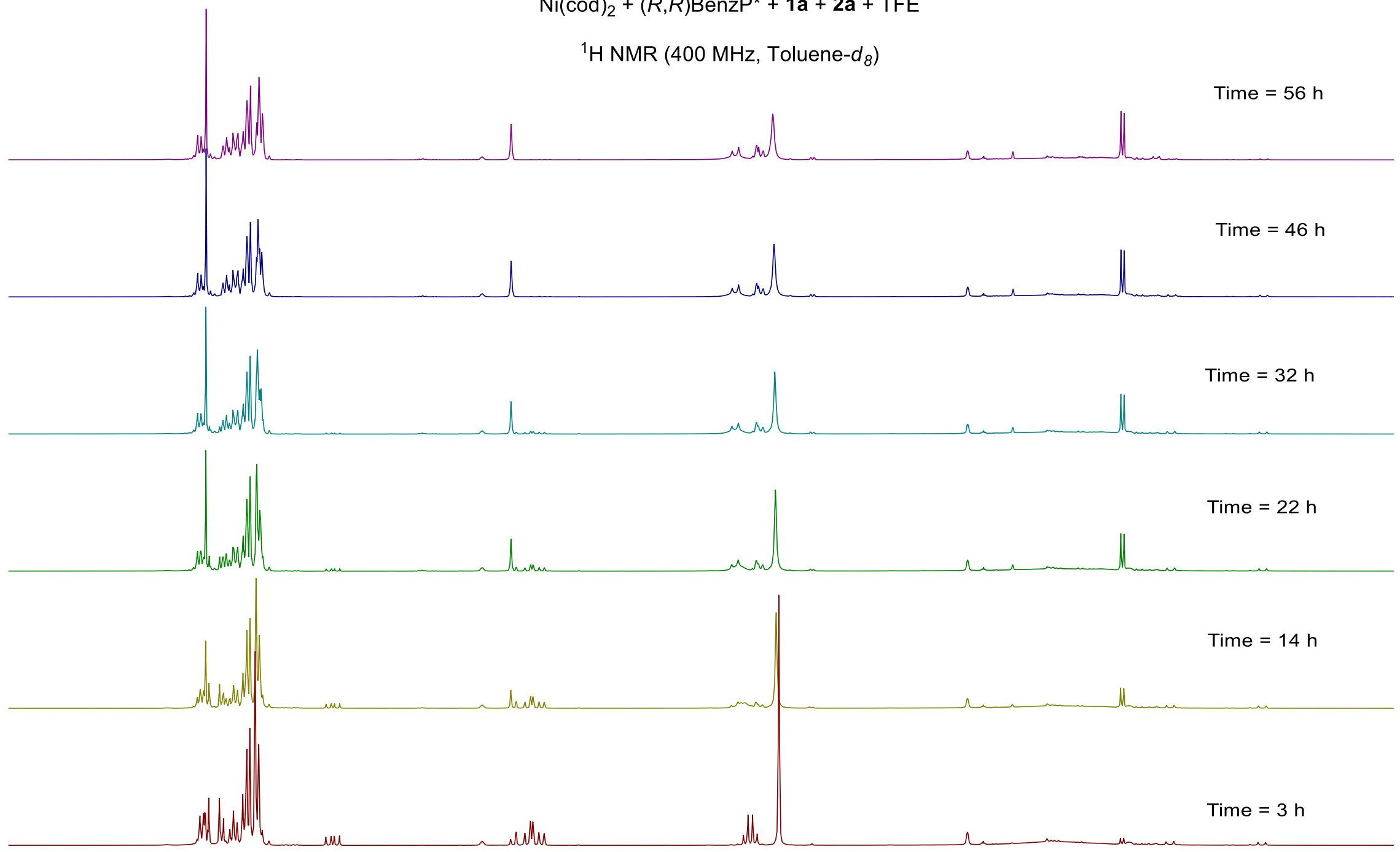

$8.5 \quad 8.0$

$7.5 \quad 7.0$

$6.5 \quad 6.0$

$5.5 \quad 5.0$

$4.5 \quad 4.0$

3.5 
Solution $\mathbf{D}(\mathrm{t}=3$ to $56 \mathrm{~h}$ )

$\mathrm{Ni}(\operatorname{cod})_{2}+(R, R)$ BenzP $^{*}+\mathbf{1 a}+\mathbf{2} \mathbf{a}+\mathrm{TFE}$

${ }^{31} \mathrm{P}$ NMR $\left(160 \mathrm{MHz}\right.$, Toluene- $\left.d_{8}\right)$

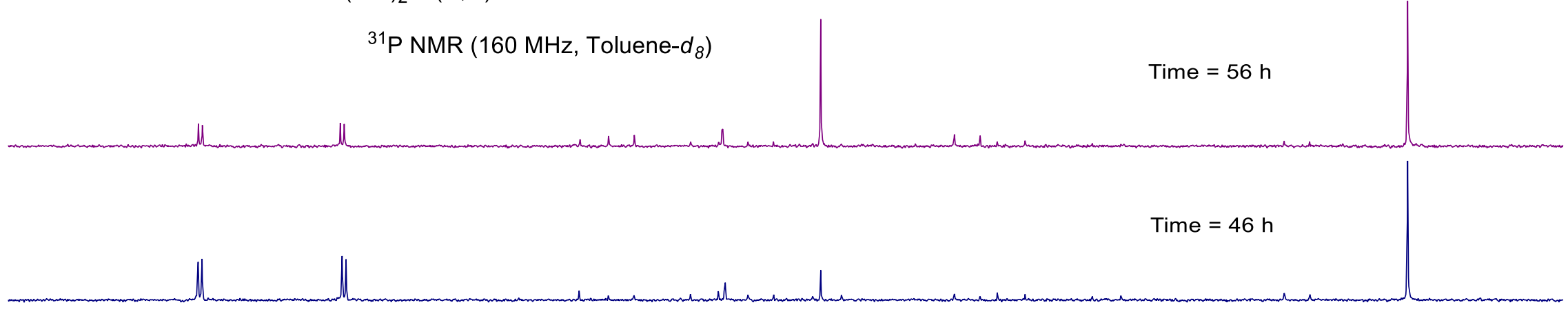

Time $=32 \mathrm{~h}$

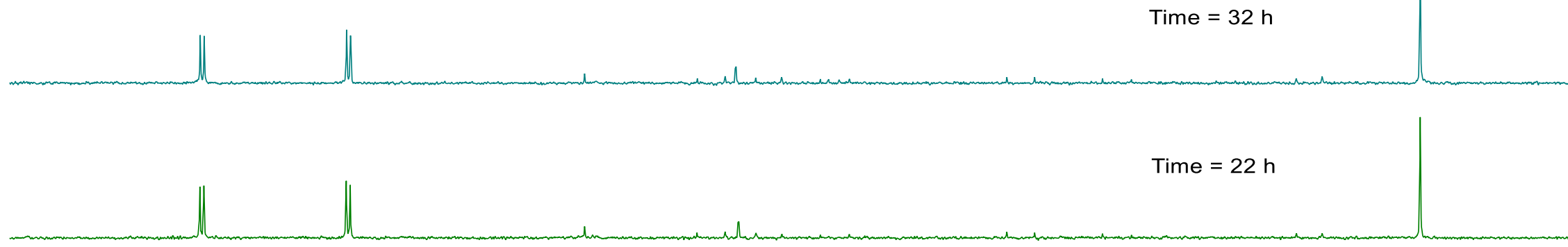

Time $=14 \mathrm{~h}$
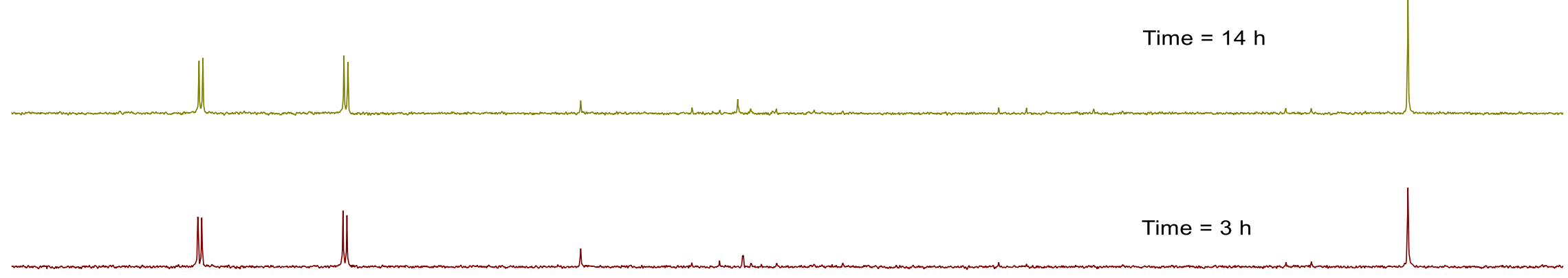

$\begin{array}{lllllllllllllllllllllllllllllllllll}65 & 64 & 63 & 62 & 61 & 60 & 59 & 58 & 57 & 56 & 55 & 54 & 53 & 52 & 51 & 50 & 49 & 48 & 47 & 46 & 45 & 44 & 43 & 42 & 41 & 40 & 39 & 38 & 37 & 36 & 35\end{array}$ 


\subsection{Synthesis of $\left[\left(\mathrm{L}_{1}\right)_{2} \mathrm{Ni}\right](7)$}

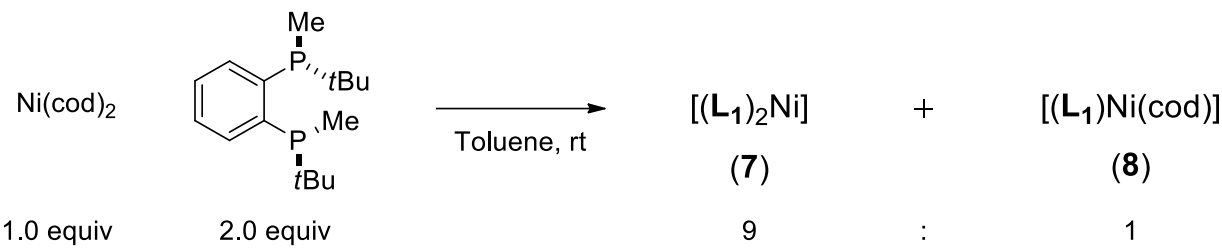

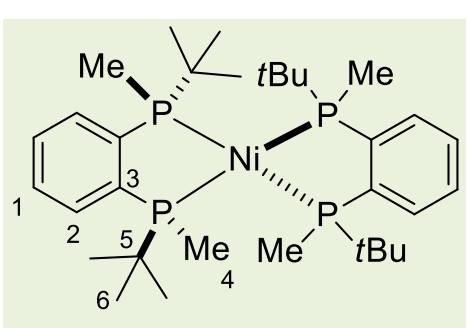

$\left[\left(\mathrm{L}_{1}\right)_{2} \mathrm{Ni}\right](\mathbf{7})$

In a $\mathrm{N}_{2}$-filled glovebox, a solution of $\mathrm{Ni}(\operatorname{cod})_{2}(13.8 \mathrm{mg}, 0.05$ mmol, 1.0 equiv) and $(R, R) B e n z P^{*} L_{1}(28.2 \mathrm{mg}, 0.10 \mathrm{mmol}, 2.0$ equiv) in toluene $(1.0 \mathrm{~mL})$ was stirred for $2 \mathrm{~h}$, then subjected to vacuum for $1 \mathrm{~h}$. The resulting red-orange oil was dissolved in toluene $(1.0 \mathrm{~mL})$, and subjected to three supplementary cycles of stirring $(1 \mathrm{~h}) /$ vacuum $(1 \mathrm{~h}) /$ dissolution (toluene, $1.0 \mathrm{~mL}$ ), leading to a mixture of homoleptic complex $\left[\left(\mathbf{L}_{1}\right)_{2} \mathrm{Ni}\right](\mathbf{7})$ and $\left[\left(L_{1}\right) \mathrm{Ni}(\mathrm{cod})\right](8)$ (red-orange oil, $33 \mathrm{mg}, 9: 1$ ratio in favor of 7 ).

${ }^{1} \mathbf{H}$ NMR $\left(500 \mathrm{MHz}\right.$, Toluene- $\left.d_{8}\right) \delta(\mathrm{ppm})=7.71-7.64(\mathrm{~m}, 2 \mathrm{H}, \mathrm{H}-1), 7.12-7.07(\mathrm{~m}, 2 \mathrm{H}, \mathrm{H}-2)$, $1.68(\mathrm{~s}, 6 \mathrm{H}, \mathrm{H}-4), 1.14-1.09(\mathrm{~m}, 9 \mathrm{H}, \mathrm{H}-6)$.

${ }^{13} \mathrm{C}\left\{{ }^{1} \mathrm{H}\right\}$ NMR $\left(130 \mathrm{MHz}\right.$, Toluene- $\left.d_{8}\right) \delta(\mathrm{ppm})=149.9-148.9(\mathrm{~m}, \mathrm{C}-3), 129.3\left(\mathrm{p}_{\mathrm{app}}, \mathrm{J}_{\mathrm{PC}}=3\right.$ $\mathrm{Hz}, \mathrm{CH}-2), 127.1(\mathrm{CH}-1), 32.2\left(p_{\text {app }}, J_{\mathrm{PC}}=7 \mathrm{~Hz}, \mathrm{C}-5\right), 28.9$ (papp, JPC $\left.=3 \mathrm{~Hz}, C_{3}-6\right), 15.8$ $\left(\mathrm{p}_{\mathrm{app}}, \mathrm{JPC}_{\mathrm{PC}}=8 \mathrm{~Hz}, \mathrm{CH}_{3}-4\right)$.

${ }^{31} \mathbf{P}\left\{{ }^{1} \mathrm{H}\right\}$ NMR $\left(160 \mathrm{MHz}\right.$, Toluene- $\left.d_{8}\right) \delta(\mathrm{ppm})=37.37$.

\subsection{Synthesis of $\left[\left(\mathrm{L}_{1}\right) \mathrm{Ni}(\mathrm{cod})\right](8)$}

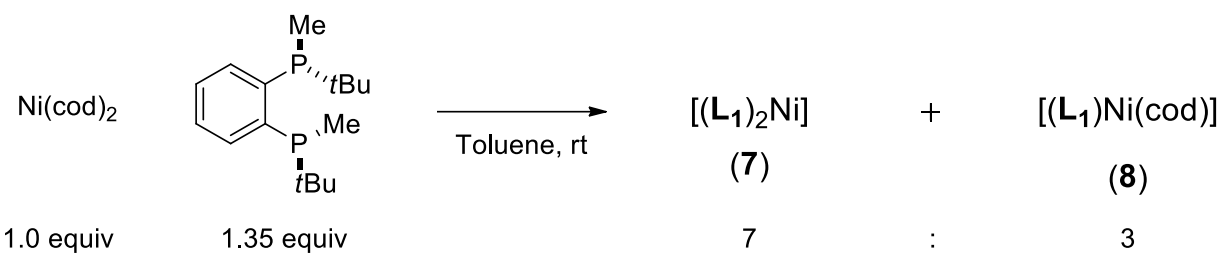

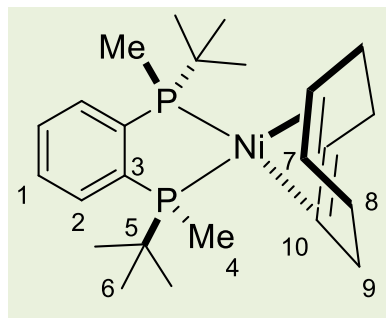

$\left[\left(\mathrm{L}_{1}\right) \mathrm{Ni}(\operatorname{cod})\right](8)$

In a $\mathrm{N}_{2}$-filled glovebox, a solution of $\mathrm{Ni}(\mathrm{cod})_{2}(6.9 \mathrm{mg}, 0.025 \mathrm{mmol}$, 1.0 equiv) and $(R, R)$ BenzP* $^{*} \mathbf{L}_{1}$ (7.8 $\mathrm{mg}, 0.0275 \mathrm{mmol}, 1.1$ equiv) in toluene $(1.0 \mathrm{~mL})$ was stirred for $2 \mathrm{~h}$, then subjected to vacuum for 1 h. The resulting red-orange oil was dissolved in toluene $(1.0 \mathrm{~mL})$, and subjected to three supplementary cycles of stirring $(1 \mathrm{~h})$ /vacuum $(1 \mathrm{~h}) /$ dissolution (toluene, $1.0 \mathrm{~mL}$ ). Additional amounts of $(R, R)$ BenzP* $^{*}(1.5 \mathrm{mg}, 0.0053 \mathrm{mmol}, 0.25$ equiv) were then added, followed by toluene (1.0 $\mathrm{mL})$ and the reaction mixture was subjected to three cycles of stirring $(1 \mathrm{~h}) /$ vacuum 
$(1 \mathrm{~h}) /$ dissolution (toluene, $1.0 \mathrm{~mL}$ ), leading to a mixture of $\left[\left(\mathbf{L}_{1}\right)_{2} \mathrm{Ni}\right](\mathbf{7})$ and $\left[\left(\mathbf{L}_{\mathbf{1}}\right) \mathrm{Ni}(\mathrm{cod})\right](\mathbf{8})$ (red-orange oil, $13 \mathrm{mg}, 7: 3$ ratio in favor of 8 ).

${ }^{1} \mathbf{H}$ NMR $\left(500 \mathrm{MHz}\right.$, Toluene- $\left.d_{8}\right) \delta(\mathrm{ppm})=7.50-7.46(\mathrm{~m}, 2 \mathrm{H}, \mathrm{H}-2), 7.11-7.06(\mathrm{~m}, 2 \mathrm{H}, \mathrm{H}-1)$, $4.48-4.33(\mathrm{~m}, 2 \mathrm{H}, \mathrm{H}-10), 4.28-4.16(\mathrm{~m}, 2 \mathrm{H}, \mathrm{H}-7), 2.85-2.77(\mathrm{~m}, 4 \mathrm{H}, \mathrm{H}-8+\mathrm{H}-9), 2.34-$ $2.24(\mathrm{~m}, 2 \mathrm{H}, \mathrm{H}-8+\mathrm{H}-9), 2.08-2.00(\mathrm{~m}, 2 \mathrm{H}, \mathrm{H}-8+\mathrm{H}-9), 1.43\left(\mathrm{~d},{ }^{2} J_{\mathrm{PH}}=5.2 \mathrm{~Hz}, 6 \mathrm{H}, \mathrm{H}-4\right)$, $0.92\left(\mathrm{~d},{ }^{3} \mathrm{JPH}_{\mathrm{PH}}=12.7 \mathrm{~Hz}, 18 \mathrm{H}, \mathrm{H}-6\right)$.

${ }^{13} \mathrm{C}\left\{{ }^{1} \mathrm{H}\right\}$ NMR $\left(130 \mathrm{MHz}\right.$, Toluene- $\left.d_{8}\right) \delta(\mathrm{ppm})=147.6-146.7(\mathrm{~m}, \mathrm{C}-3), 130.2\left(\mathrm{t}_{\mathrm{app}}, \mathrm{J}_{\mathrm{PC}}=7\right.$ $\mathrm{Hz}, \mathrm{CH}-2), 127.8(\mathrm{CH}-1), 82.8(\mathrm{CH}-7), 81.9\left(\mathrm{t}_{\mathrm{app}}, \mathrm{J}_{\mathrm{PC}}=4 \mathrm{~Hz}, \mathrm{CH}-10\right), 37.5\left(\mathrm{t}_{\mathrm{app}}, \mathrm{J}_{\mathrm{PC}}=6 \mathrm{~Hz}\right.$, $\left.\mathrm{CH}_{2}-8\right)$, $32.31-31.95(\mathrm{~m}, \mathrm{C}-5), 28.1\left(\mathrm{CH}_{2}-9\right), 27.0$ (tapp, $\left.\mathrm{JPC}_{\mathrm{PC}}=4 \mathrm{~Hz}, \mathrm{CH}_{3}-6\right), 8.28\left(\mathrm{t}_{\mathrm{app}}, \mathrm{J}_{\mathrm{PC}}=\right.$ $\left.8.1 \mathrm{~Hz}, \mathrm{CH}_{3}-4\right)$

${ }^{31} \mathrm{P}\left\{{ }^{1} \mathrm{H}\right\}$ NMR $\left(160 \mathrm{MHz}\right.$, Toluene- $\left.d_{8}\right) \delta(\mathrm{ppm})=49.26$.

\subsection{Synthesis of $\left[\left(L_{1}\right) \mathrm{Ni}(1 \mathrm{a})\right](9)$}

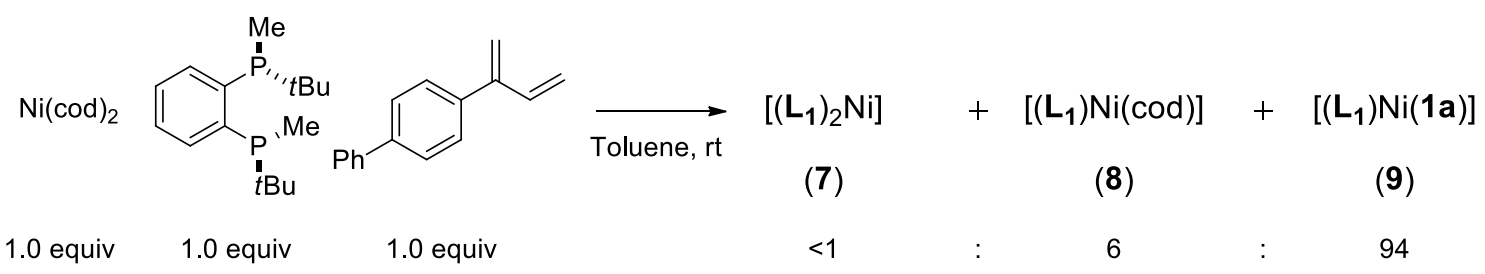

In a $\mathrm{N}_{2}$-filled glovebox, a solution of $\mathrm{Ni}(\operatorname{cod})_{2}(27.5 \mathrm{mg}, 0.10 \mathrm{mmol}, 1.0$ equiv) and $(R, R)$ BenzP $^{*} \mathrm{~L}_{1}$ (28.2 mg, $0.10 \mathrm{mmol}, 1.0$ equiv) and 4-(buta-1,3-dien-2-yl)-1,1'-biphenyl 1a (21.1 $\mathrm{mg}, 0.10 \mathrm{mmol}, 1.0$ equiv) in toluene $(1.0 \mathrm{~mL})$ was stirred for $0.5 \mathrm{~h}$, then subjected to high vacuum for $1 \mathrm{~h}$. The resulting red-orange oil was dissolved in toluene $(1.0 \mathrm{~mL})$, and subjected to ten supplementary cycles of stirring $(1 \mathrm{~h}) /$ vacuum $(1 \mathrm{~h}) /$ dissolution (toluene, 1.0 $\mathrm{mL}$ ), leading to a mixture of $\left[\left(\mathbf{L}_{1}\right) \mathrm{Ni}(\operatorname{cod})\right](\mathbf{8})$ and $\left[\left(\mathbf{L}_{1}\right) \mathrm{Ni}(\mathbf{1 a})\right](\mathbf{9})$ (red-orange oil, $56.0 \mathrm{mg}$, 94:6 ratio in favor of 9 ). Residual peaks from diene $\mathbf{1 a}$ and $\left[\left(\mathbf{L}_{1}\right)_{2} \mathrm{Ni}\right](\mathbf{7})$ were also present. Due to the fluxional nature of the complex at $r t$, all $1 D$ and $2 D$ NMR spectra presented numerous weak and/or broad signals. Some carbons, such as olefinic carbons 13-16, were not even observed. Moreover, the presence of residual $\left[\left(\mathbf{L}_{\mathbf{1}}\right) \mathrm{Ni}(\mathrm{cod})\right](\mathbf{8})$ further complicates the interpretation of the NMR spectra. Nevertheless, we propose a tentative assignment for some of the most well defined peaks, but urge the reader to take them with caution.

${ }^{1} \mathbf{H}$ NMR $\left(500 \mathrm{MHz}\right.$, Toluene- $\left.d_{8}\right) \delta(\mathrm{ppm})=7.71\left(\mathrm{~d},{ }^{3} \mathrm{~J}_{\mathrm{HH}}=8.1 \mathrm{~Hz}, 2 \mathrm{H}, \mathrm{H}-18\right), 7.48(\mathrm{~m}, \mathrm{H}-A r)$, $7.22\left(\mathrm{t},{ }^{3} \mathrm{~J}_{\mathrm{HH}}=7.5 \mathrm{~Hz}, 2 \mathrm{H}, \mathrm{H}-A r\right), 5.22(\mathrm{~s}, 1 \mathrm{H}, \mathrm{C}=\mathrm{C}-H), 4.09(\mathrm{~s}, 1 \mathrm{H}, \mathrm{C}=\mathrm{C}-H), 3.66(\mathrm{~s}, 1 \mathrm{H}, \mathrm{C}=\mathrm{C}-$ $H$ ), $3.09(\mathrm{~s}, 1 \mathrm{H}, \mathrm{C}=\mathrm{C}-H), 2.83(\mathrm{~s}, 1 \mathrm{H}, \mathrm{C}=\mathrm{C}-H), 1.46$ (d, ${ }^{2} \mathrm{JPH}_{\mathrm{PH}}=5.4 \mathrm{~Hz}, 3 \mathrm{H}, \mathrm{H}-9$ or H-12), 1.17 $\left(\mathrm{d},{ }^{2} J_{\mathrm{PH}}=3.9 \mathrm{~Hz}, 3 \mathrm{H}, \mathrm{H}-9\right.$ or H-12), $0.95\left(\mathrm{~d},{ }^{3} J_{\mathrm{PH}}=13.4 \mathrm{~Hz}, 9 \mathrm{H}, \mathrm{H}-8\right.$ or H-11), $0.82\left(\mathrm{~d},{ }^{3} J_{\mathrm{PH}}=\right.$ $13.4 \mathrm{~Hz}, 9 \mathrm{H}, \mathrm{H}-8$ or $\mathrm{H}-11)$. 


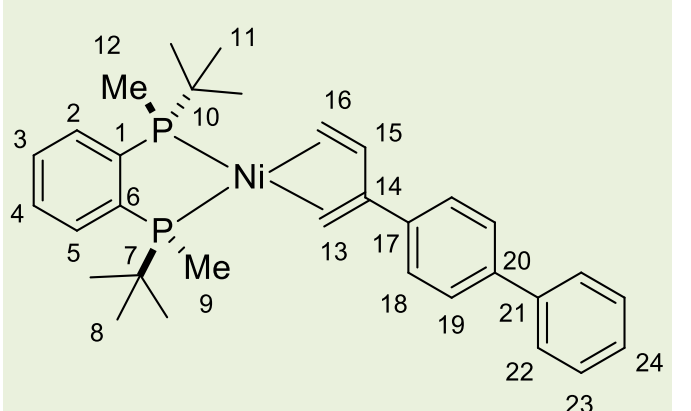

$\left[\left(\mathbf{L}_{\mathbf{1}}\right) \mathrm{Ni}(\mathbf{1} \mathbf{a})\right](\mathbf{9})$

${ }^{13} \mathrm{C}\left\{{ }^{1} \mathrm{H}\right\}$ NMR $\left(130 \mathrm{MHz}\right.$, Toluene- $\left.d_{8}\right) \delta(\mathrm{ppm})=$ 147.6 - 145.8 (C-1 or $C-6$ ), 144.4 (C-Ar), 141.8 (CAr), $138.3(C-A r), 130.6\left(\mathrm{~d},{ }^{1} \mathrm{JPC}_{\mathrm{PC}}=11 \mathrm{~Hz}, \mathrm{CH}-2\right.$ or $\mathrm{CH}-5), 130.1\left(\mathrm{~d},{ }^{1} \mathrm{JP}_{\mathrm{PC}}=11 \mathrm{~Hz}, \mathrm{CH}-2\right.$ or $\left.\mathrm{CH}-5\right)$, 129.2 ( $\mathrm{CH}-\mathrm{Ar}), 128.9$ (CH-Ar), $128.4(\mathrm{CH}-\mathrm{Ar})$, 127.6- $127.1(\mathrm{~m}, \mathrm{CH}-\mathrm{Ar}), 127.1(\mathrm{CH}-\mathrm{Ar}), 127.0$ ( $\mathrm{CH}-\mathrm{Ar}), 126.9(\mathrm{CH}-\mathrm{Ar}), 126.5(\mathrm{CH}-\mathrm{Ar}), 32.7-32.2$ $(\mathrm{m}, C-7$ or $C-10), 31.9\left(\mathrm{dd},{ }^{1} \mathrm{~J}_{\mathrm{PC}}=16,{ }^{4} \mathrm{~J}_{\mathrm{PC}}=4 \mathrm{~Hz}\right.$, C-7 or C-10), 27.4 (tapp, $\mathrm{JPC}_{\mathrm{PC}}=7 \mathrm{~Hz}, \mathrm{CH}_{3}-8$ and $\left.\mathrm{CH}_{3}-11\right), 9.9\left(\mathrm{CH}_{3}-9\right.$ or $\left.\mathrm{CH}_{3}-12\right), 7.1\left(\mathrm{CH}_{3}-9\right.$ or $\left.\mathrm{CH}_{3}-12\right)$.

${ }^{31} \mathrm{P}\left\{{ }^{1} \mathrm{H}\right\}$ NMR $\left(160 \mathrm{MHz}\right.$, Toluene- $\left.d_{8}\right) \delta(\mathrm{ppm})=57.91$ (broad), 40.44 (broad).

\subsection{Synthesis of $\left[\left(L_{1}\right) \mathrm{Ni}\left(\eta^{3}\right.\right.$-allyl $\left.)\right]\left[\mathrm{OCH}_{2} \mathrm{CF}_{3}\right](10)$}

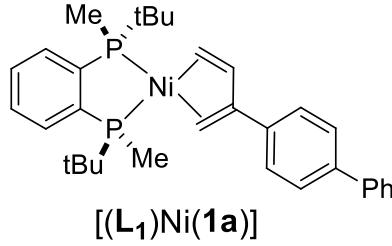

(9)

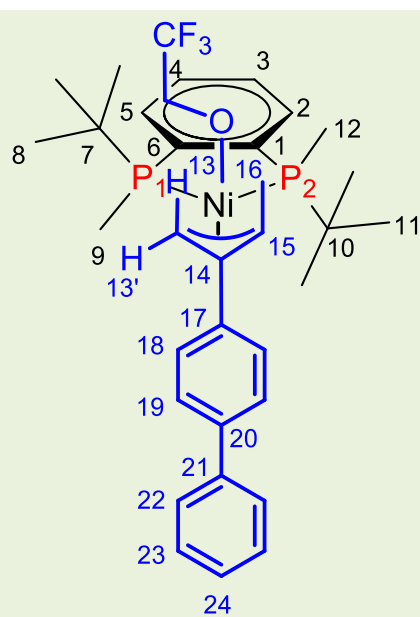

$\left[\left(\mathrm{L}_{1}\right) \mathrm{Ni}\left(\eta^{3}\right.\right.$-allyl $\left.)\right]\left[\mathrm{OCH}_{2} \mathrm{CF}_{3}\right](\mathbf{1 0})$

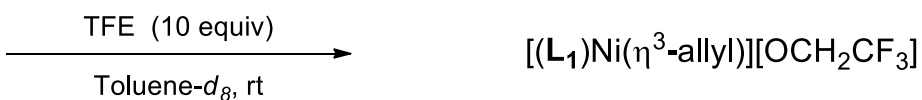

(10)

In a $\mathrm{N}_{2}$-filled glovebox, a $2 \mathrm{~mL}$ vial was charged

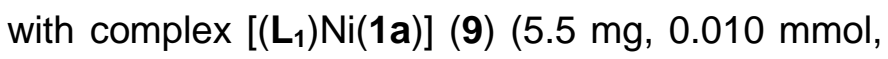
1.0 equiv), followed by toluene- $d_{8}(0.60 \mathrm{~mL})$. To the resulting orange solution was added a $1.0 \mathrm{M}$ solution of $\mathrm{CF}_{3} \mathrm{CH}_{2} \mathrm{OH}$ in toluene- $d_{8}(0.10 \mathrm{~mL}, 0.10$ mmol, 10.0 equiv). The solution immediately changed color to pale orange/yellow. $0.40 \mathrm{~mL}$ of this solution was introduced in a J-Young tube and analyzed by NMR.

Due to the reversible nature of the reaction, the complex $\left[\left(\mathrm{L}_{\mathbf{1}}\right) \mathrm{Ni}\left(\eta^{3}\right.\right.$-allyl) $]\left[\mathrm{OCH}_{2} \mathrm{CF}_{3}\right](\mathbf{1 0})$ could only be observed in solution. Indeed, in vaccum concentration of this solution resulted in quantitative regeneration of the starting complex $\left[\left(\mathbf{L}_{1}\right) \mathrm{Ni}(\mathbf{1} \mathbf{a})\right](\mathbf{9})$.

${ }^{1} \mathbf{H}$ NMR $\left(500 \mathrm{MHz}\right.$, Toluene- $\left.d_{8}\right) \delta(\mathrm{ppm})=7.49-7.31(\mathrm{~m}, 8 \mathrm{H}, \mathrm{H}-A r), 7.23\left(\mathrm{t},{ }^{3} \mathrm{JHH}_{\mathrm{HH}}=7.7 \mathrm{~Hz}\right.$, $2 \mathrm{H}, \mathrm{H}-A r), 7.16-7.12(\mathrm{~m}, 1 \mathrm{H}, \mathrm{H}-24), 7.11-7.09(\mathrm{~m}, 2 \mathrm{H}, \mathrm{H}-18), 5.43-5.20(\mathrm{~m}, 1 \mathrm{H}, \mathrm{H}-15)$, $3.90-3.86\left(\mathrm{~m}, 1 \mathrm{H}, \mathrm{H}-13^{\prime}\right), 2.72-2.67(\mathrm{~m}, 1 \mathrm{H}, \mathrm{H}-13), 1.54\left(\mathrm{~d},{ }^{2} \mathrm{~J}_{\mathrm{PH}}=8.5 \mathrm{~Hz}, 3 \mathrm{H}, \mathrm{H}-9\right), 1.50$ 
(d, $\left.{ }^{2} J_{\mathrm{PH}}=8.5 \mathrm{~Hz}, 3 \mathrm{H}, \mathrm{H}-12\right), 0.90-0.86(\mathrm{~m}, 3 \mathrm{H}, \mathrm{H}-16), 0.80\left(\mathrm{~d},{ }^{3} J_{\mathrm{PH}}=15.6 \mathrm{~Hz}, 9 \mathrm{H}, \mathrm{H}-8\right)$, $0.16\left(\mathrm{~d},{ }^{3} \mathrm{~J}_{\mathrm{PH}}=15.6 \mathrm{~Hz}, 9 \mathrm{H}, \mathrm{H}-11\right)$.

${ }^{13} \mathbf{C}\left\{{ }^{1} \mathrm{H}\right\}$ NMR $\left(130 \mathrm{MHz}\right.$, Toluene- $\left.d_{8}\right) \delta(\mathrm{ppm})=143.3(C-20), 139.8(C-21), 139.5-138-5$ (m, $C-1$ and $C-6), 137.1(C-17), 132.3-131.9(C H-2$ or $C H-5), 131.8\left(\mathrm{~d},{ }^{2} J_{\mathrm{PP}}=14 \mathrm{~Hz}, \mathrm{CH}-2\right.$ or $\mathrm{CH}-5), 129.3(\mathrm{CH}-22), 128.4(\mathrm{CH}-24), 127.6(\mathrm{CH}-3$ or $\mathrm{CH}-4), 127.2(\mathrm{CH}-3$ or $\mathrm{CH}-4), 123.1$ (C-14), 76.7 (d, $\left.{ }^{2} J_{\mathrm{PP}}=15 \mathrm{~Hz}, \mathrm{CH}-15\right), 55.2\left(\mathrm{dd},{ }^{2} J_{\mathrm{PP}}=19,3 \mathrm{~Hz}, \mathrm{CH}_{2}-13\right), 32.9$ (d, ${ }^{2} J_{\mathrm{PP}}=14$ $\mathrm{Hz}, C-7$ or C-10), 32.7 (d, ${ }^{1} \mathrm{JPP}_{\mathrm{PP}}=13 \mathrm{~Hz}, C-7$ or $C-10$ ), 26.9 (d, ${ }^{2} \mathrm{JPP}_{\mathrm{PP}} 5 \mathrm{~Hz}, \mathrm{CH}_{3}-8$ ), 25.9 (d, $\left.{ }^{2} J_{\mathrm{PP}}=5 \mathrm{~Hz}, \mathrm{CH}_{3}-11\right), 14.8\left(\mathrm{CH}_{3}-16\right), 7.7\left(\mathrm{~d},{ }^{1} \mathrm{JPP}_{\mathrm{PP}}=25 \mathrm{~Hz}, \mathrm{CH}_{3}-9\right), 5.3\left(\mathrm{~d},{ }^{1} \mathrm{JPP}_{\mathrm{PP}}=24 \mathrm{~Hz}, \mathrm{CH}_{3}-\right.$ 12).

${ }^{31} \mathrm{P}\left\{{ }^{1} \mathrm{H}\right\}$ NMR $\left(160 \mathrm{MHz}\right.$, Toluene- $\left.d_{8}\right) \delta(\mathrm{ppm})=61.51\left(\mathrm{~d},{ }^{3} \mathrm{JPP}=9.7 \mathrm{~Hz}, \mathrm{P}-1\right), 58.58\left(\mathrm{~d},{ }^{3} \mathrm{JPP}_{\mathrm{PP}}=\right.$ 9.7 Hz, P-2).

${ }^{19} \mathrm{~F}\left\{{ }^{1} \mathrm{H}\right\}$ NMR $\left(280 \mathrm{MHz}\right.$, Toluene- $\left.d_{8}\right) \delta(\mathrm{ppm})=-76.87$.

\subsection{Synthesis of $\left[\left(L_{1}\right) \mathrm{Ni}(3 \mathrm{aa})\right](11)$}

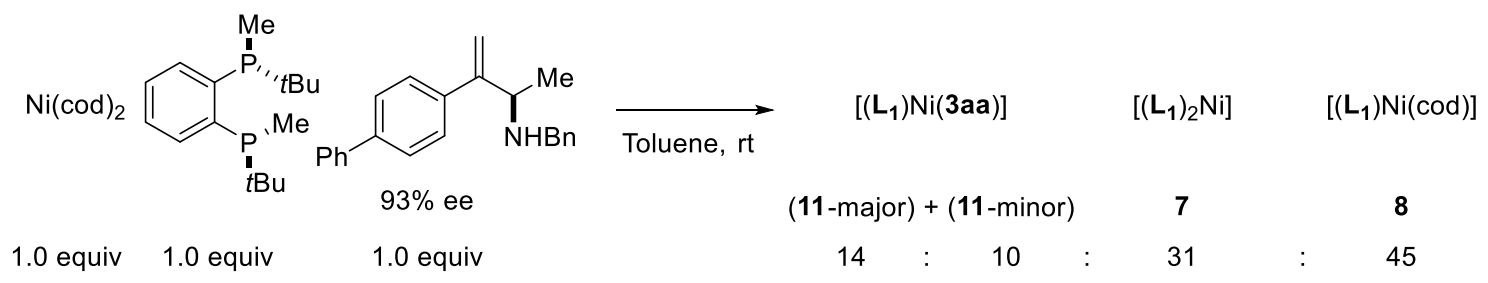

In a $\mathrm{N}_{2}$-filled glovebox, to a solution of $\mathrm{Ni}(\operatorname{cod})_{2}(8.8 \mathrm{mg}, 0.032 \mathrm{mmol}, 1.0$ equiv) and $(R, R)$ BenzP* $^{*} L_{1}(9.0 \mathrm{mg}, 0.032 \mathrm{mmol}, 1.0$ equiv) in toluene $(0.50 \mathrm{~mL})$ was added 3aa $(10.0$ $\mathrm{mg}, 0.032 \mathrm{mmol}, 1.0$ equiv, 93\% ee). The resulting solution was stirred for $1.0 \mathrm{~h}$, then subjected to high vacuum for $1 \mathrm{~h}$. The resulting red-orange oil was dissolved in toluene (1.0 $\mathrm{mL})$, and subjected to ten supplementary cycles of stirring $(1 \mathrm{~h})$ /vacuum $(1 \mathrm{~h}) /$ dissolution (toluene, $1.0 \mathrm{~mL}$ ), leading to a mixture of two product-bound complexes $\left[\left(\mathbf{L}_{\mathbf{1}}\right) \mathrm{Ni}(\mathbf{3 a a})\right](\mathbf{1 1}$ major) and $\left[\left(\mathbf{L}_{\mathbf{1}}\right) \mathrm{Ni}(\mathbf{3 a a})\right]\left(\mathbf{1 1}\right.$-minor), along with $\left[\left(\mathbf{L}_{1}\right)_{2} \mathrm{Ni}\right](\mathbf{7})$ and $\left[\left(\mathbf{L}_{\mathbf{1}}\right) \mathrm{Ni}(\mathrm{cod})\right](\mathbf{8})$ (red-orange oil, $18.0 \mathrm{mg}$, ratio 11-major/11-minor/7/8=14:10:31:45).

Due to the complexity of mixture, it was not possible to fully attribute the ${ }^{1} \mathrm{H}$ and ${ }^{13} \mathrm{C} \mathrm{NMR}$ peaks present in the spectrum. Nevertheless, we are confident that the observed peaks and correlations for 11-major are consistent with a $\left[\left(\mathbf{L}_{1}\right) \mathrm{Ni}(3 \mathbf{a a})\right]$ complex bound through the olefin $\pi$-system of 3aa. Whether the nitrogen is bound or not is unclear. Only the peaks that could be attributed with confidence to this complex are reported. Due to numerous overlaps, we were not able to perform such an analysis for 11-minor, and the corresponding peaks are not reported. 


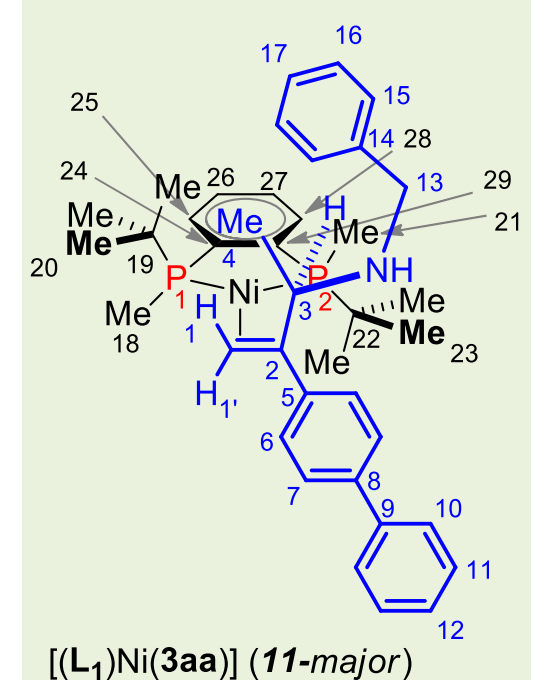

$\left[\left(\mathrm{L}_{1}\right) \mathrm{Ni}(3 \mathrm{aa})\right]$ (11-major)

${ }^{1} \mathbf{H}$ NMR $\left(500 \mathrm{MHz}\right.$, Toluene- $\left.d_{8}\right) \delta(\mathrm{ppm})=4.31-4.25(\mathrm{~m}$, $1 \mathrm{H}, \mathrm{H}-3), 4.07-4.02(\mathrm{~m}, 1 \mathrm{H}, \mathrm{H}-13), 3.89-3.84(\mathrm{~m}, 1 \mathrm{H}, \mathrm{H}-$ 13), 3.25 (qapp, $J=4.5 \mathrm{~Hz}, 1 \mathrm{H}, \mathrm{H}-1$ ), 2.53 (dtapp, $J=7.7,4.1$ $\mathrm{Hz}, 1 \mathrm{H}, \mathrm{H}-1), 1.65\left(\mathrm{~d},{ }^{3} J_{\mathrm{HH}}=5.8 \mathrm{~Hz}, 3 \mathrm{H}, \mathrm{H}-4\right), 1.29\left(\mathrm{~d},{ }^{2} J_{\mathrm{PH}}=\right.$ $4.5 \mathrm{~Hz}, 3 \mathrm{H}, \mathrm{H}-18), 1.01$ (d, $\left.{ }^{3} \mathrm{JPH}_{\mathrm{PH}}=13.2 \mathrm{~Hz}, 9 \mathrm{H}, \mathrm{H}-20\right), 0.78$ (d, $\left.{ }^{3} J_{\mathrm{PH}}=13.5 \mathrm{~Hz}, 9 \mathrm{H}, \mathrm{H}-23\right), 0.63\left(\mathrm{~d},{ }^{2} \mathrm{~J}_{\mathrm{PH}}=5.2 \mathrm{~Hz}, 3 \mathrm{H}, \mathrm{H}-\right.$ 21).

${ }^{13} \mathrm{C}\left\{{ }^{1} \mathrm{H}\right\}$ NMR $\left(130 \mathrm{MHz}\right.$, Toluene- $\left.d_{8}\right) \delta(\mathrm{ppm})=70.8-70.5$ (m, C-2), 57.7 - $57.6(\mathrm{~m}, \mathrm{CH}-3), 49.1\left(\mathrm{CH}_{2}-13\right), 34.8$ (dd, $\left.{ }^{2} J_{\mathrm{PP}}=22,2 \mathrm{~Hz}, \mathrm{CH}_{2}-1\right), 27.5\left(\mathrm{dd},{ }^{2} J_{\mathrm{PP}}=8,{ }^{4} \mathrm{JPP}_{\mathrm{PP}}=3 \mathrm{~Hz}, \mathrm{CH}_{3}\right.$ 20), $27.3\left(\mathrm{~d},{ }^{2} \mathrm{JPP}_{\mathrm{PP}}=9 \mathrm{~Hz}, \mathrm{CH}_{3}-23\right), 21.6\left(\mathrm{CH}_{3}-4\right), 7.9$ (dd, ${ }^{1} J_{\mathrm{PP}}$ $\left.=11,{ }^{4} J_{\mathrm{PP}}=8 \mathrm{~Hz}, \mathrm{CH}_{3}-18\right), 4.7\left(\mathrm{dd},{ }^{1} \mathrm{JPP}_{\mathrm{PP}}=12,{ }^{4} \mathrm{JPP}_{\mathrm{PP}}=5 \mathrm{~Hz}, \mathrm{CH}_{3}-21\right)$.

${ }^{31} \mathrm{P}\left\{{ }^{1} \mathrm{H}\right\}$ NMR $\left(160 \mathrm{MHz}\right.$, Toluene- $\left.d_{8}\right) \delta(\mathrm{ppm})=51.58\left(\mathrm{~d},{ }^{3} J_{\mathrm{PP}}=69.4 \mathrm{~Hz}, \mathrm{P}-1\right), 45.33\left(\mathrm{~d},{ }^{3} J_{\mathrm{PP}}\right.$ $=69.4 \mathrm{~Hz}, \mathrm{P}-2)$. 


\subsection{Reaction of $\left[\left(\mathrm{L}_{1}\right) \mathrm{Ni}\left(\eta^{3}\right.\right.$-allyl $\left.)\right]\left[\mathrm{OCH}_{2} \mathrm{CF}_{3}\right](10)$ with $\mathrm{BnNH}_{2}$}

In a $\mathrm{N}_{2}$-filled glovebox, a $2 \mathrm{~mL}$ vial was charged with complex [( $\left.\left.\mathbf{L}_{\mathbf{1}}\right) \mathrm{Ni}(\mathbf{1} \mathbf{a})\right](\mathbf{9})(5.5 \mathrm{mg}, 0.010$ mmol, 1.0 equiv), followed by toluene- $d_{8}(0.60 \mathrm{~mL})$. To the resulting orange solution was added a $1.0 \mathrm{M}$ solution of $\mathrm{CF}_{3} \mathrm{CH}_{2} \mathrm{OH}$ in toluene- $d_{8}(0.10 \mathrm{~mL}, 0.10 \mathrm{mmol}, 10.0$ equiv). The solution immediately changed color to pale orange/yellow. $\mathrm{BnNH}_{2}(43.0 \mathrm{mg}, 0.40 \mathrm{mmol}, 40.0$ equiv) was then added, and the reaction mixture was stirred for 15 min (solution E). $0.40 \mathrm{~mL}$ of this solution was introduced in a J-Young tube and analyzed by ${ }^{1} \mathrm{H}$ and ${ }^{31} \mathrm{P}\left\{{ }^{1} \mathrm{H}\right\} \mathrm{NMR}$. 


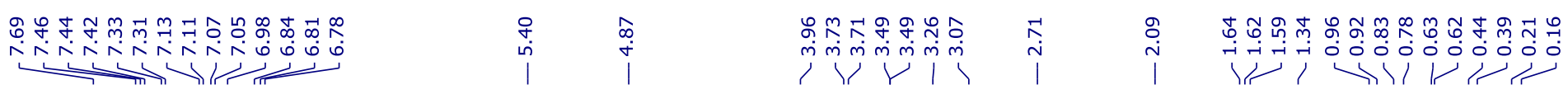

Solution $\mathrm{E}$

Complex (10) +40 equiv $\mathrm{BnNH}_{2}$

${ }^{1} \mathrm{H}$ NMR (400 MHz, Toluene- $d_{8}$ )
- $\quad$ * Tol- $d_{n}$

- Complex $\left[\left(\mathrm{L}_{1}\right)_{2} \mathrm{Ni}\right](7)$

A. Complex $\left[\left(\mathrm{L}_{1}\right) \mathrm{Ni}\left(\eta^{3}\right.\right.$-allyl) $]\left[\mathrm{OCH}_{2} \mathrm{CF}_{3}\right](10)$

$\otimes$ Complex $\left[\left(\mathrm{L}_{1}\right) \mathrm{Ni}(3 \mathrm{aa})\right]$ (11-major $)$

$\otimes$ Complex $\left[\left(\mathrm{L}_{1}\right) \mathrm{Ni}(3 \mathrm{aa})\right]$ (11-minor)

- $\mathrm{CF}_{3} \mathrm{CH}_{2} \mathrm{OH}$

- $\mathrm{BnNH}_{2}$

$\underset{\substack{i \\ i \\ i}}{i}$

$\stackrel{\substack{\text { I } \\ \dot{+}}}{+}$

\section{- \\ -}




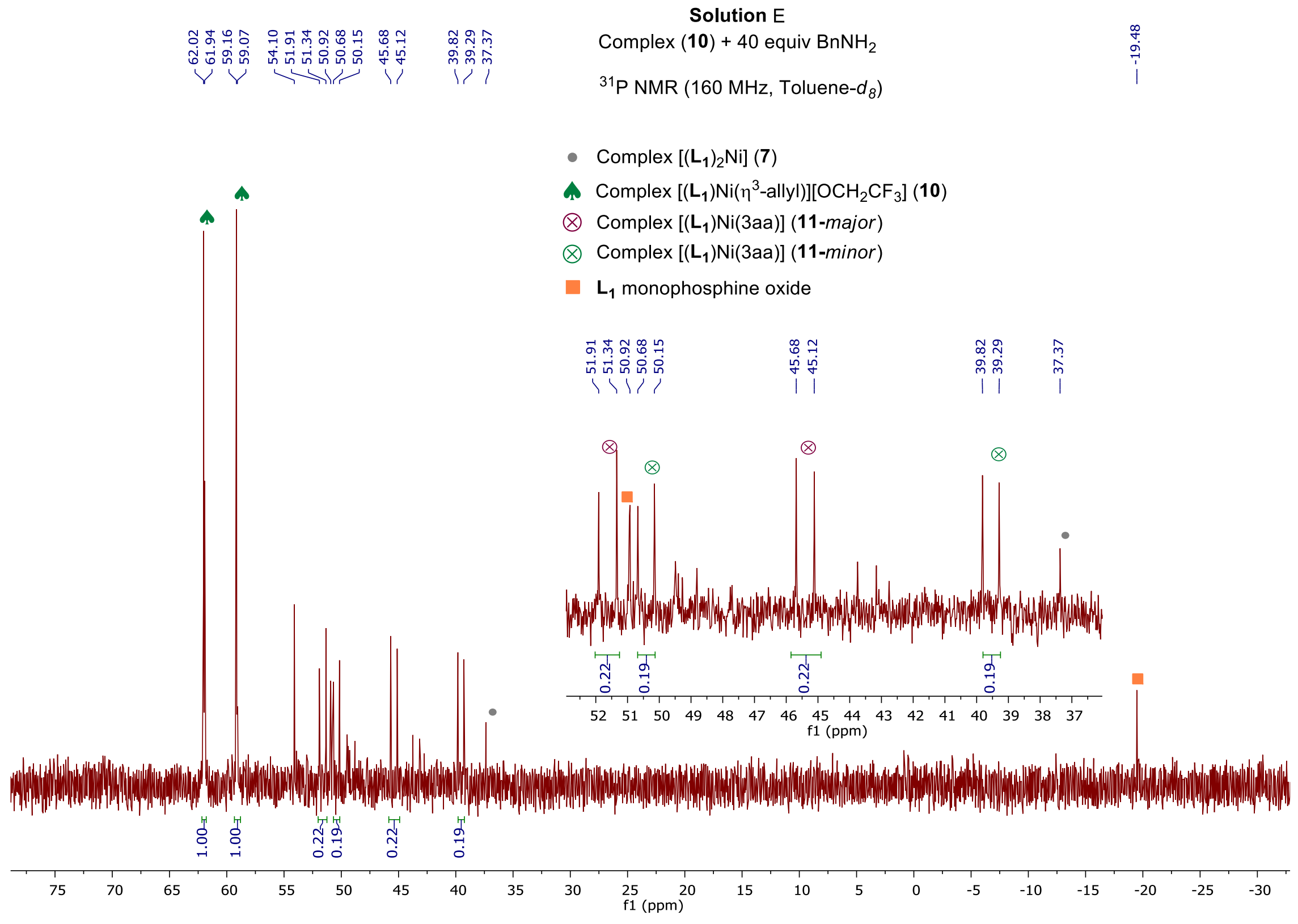




\section{Amine exchange experiment}
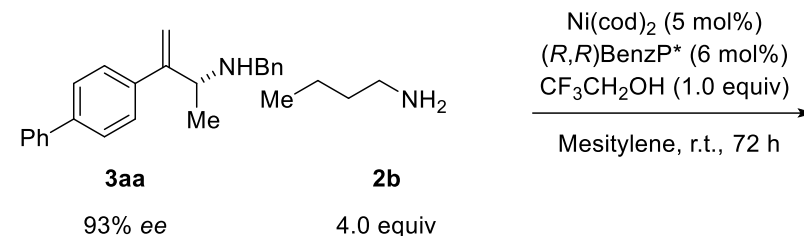

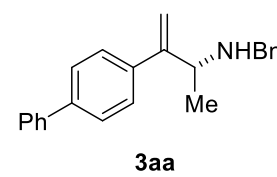

$>99 \%$ recovery $93 \%$ ee

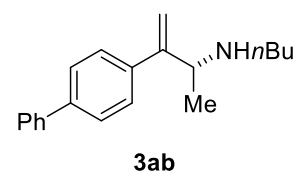

not observed

In a $\mathrm{N}_{2}$-filled glovebox, $\mathrm{Ni}(\mathrm{cod})_{2}(1.4 \mathrm{mg}, 0.005 \mathrm{mmol}, 5 \mathrm{~mol} \%)$ and $(R, R)$-BenzP ${ }^{*} \mathrm{~L}_{1}(1.7$ $\mathrm{mg}, 0.006 \mathrm{mmol}, 6 \mathrm{~mol} \%$ ) were weighted in a $5 \mathrm{~mL}$ Schlenk tube, and anhydrous mesitylene $(0.20 \mathrm{~mL}, 0.5 \mathrm{M})$ was added. After stirring at room temperature for $10 \mathrm{~min}$, allylic amine 3aa (31.0 mg, 0.10 mmol, 1.0 equiv, $93 \%$ ee), $n \mathrm{BuNH}_{2} \mathbf{2 b}(40 \mu \mathrm{L}, 0.40 \mathrm{mmol}, 4.0$ equiv) and TFE ( $7 \mu \mathrm{L}, 0.10 \mathrm{mmol}, 1.0$ equiv) were added sequentially. The tube was sealed, taken out of the glovebox and stirred at room temperature for $72 \mathrm{~h}$. The reaction mixture was filtered over a short pad of silica gel, washed with ethyl acetate $(10 \mathrm{~mL})$ and concentrated under vacuum to afford the crude mixture. Analysis by ${ }^{1} \mathrm{H}$ NMR showed $>99 \%$ recovery of 3aa and no traces of product $3 \mathbf{a b}$. 


\section{Deuterium labeling experiments}

\subsection{Model catalytic hydroamination using TFE- $d_{1}$ and $B n N_{2}$}

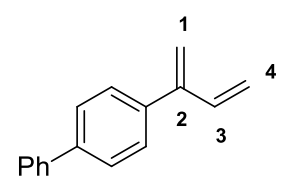

$1 \mathrm{a}$

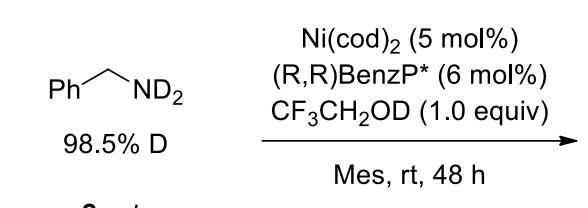

$2 \mathrm{a}-\mathrm{d}_{2}$
4.0 equiv \begin{tabular}{ll|l|lll} 
& & & \multicolumn{3}{|c}{ Deuterium Incorporation } \\
Diene & Yield & ee & C1 & C3 & C4 \\
Cons. & & & & & \\
\hline$>95 \%$ & $76 \%$ & $90 \%$ & $57.5 \%$ & $<2 \%$ & $58 \%$
\end{tabular}

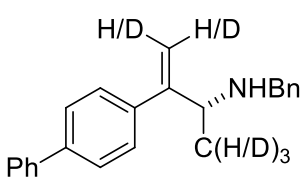

$(R)-3$ aa- $d_{n}$

In a $\mathrm{N}_{2}$-filled glovebox, $\mathrm{Ni}(\mathrm{cod})_{2}(3.4 \mathrm{mg}, 0.0125 \mathrm{mmol}, 5 \mathrm{~mol} \%)$ and $(R, R)$-BenzP ${ }^{\star} \mathrm{L}_{\mathbf{1}}(4.2$ $\mathrm{mg}, 0.015 \mathrm{mmol}, 6 \mathrm{~mol} \%$ ) were weighted in a $5 \mathrm{~mL}$ Schlenk tube, and anhydrous mesitylene $(0.50 \mathrm{~mL}, 0.5 \mathrm{M})$ was added. After stirring at room temperature for $10 \mathrm{~min}$, diene $1 \mathrm{a}$ (42.0 $\mathrm{mg}, 0.25 \mathrm{mmol}, 1.0$ equiv), TFE- $d_{1}\left(26.0 \mathrm{mg}, 0.25 \mathrm{mmol}, 1.0\right.$ equiv, $99.5 \%$ D) and $B n-\mathrm{ND}_{2}$ (110.0 mg, $1.00 \mathrm{mmol}, 4.0$ equiv, 98.5\% D) were added sequentially. The tube was sealed, taken out of the glovebox and stirred at room temperature for $48 \mathrm{~h}$. The reaction mixture was filtered over a short pad of silica gel, washed with ethyl acetate $(10 \mathrm{~mL})$ and concentrated under vacuum to afford the crude mixture. Consumption of 1a: $>95 \%$. Purification by flash chromatography over silica gel (pentane:EtOAc $=4: 1$ ) led to the desired product 3aa- $d_{n}$ as a pale yellow oil $\left(60.0 \mathrm{mg}, 76 \%\right.$ yield, $90 \%$ ee). ${ }^{1} \mathrm{H}$ NMR analysis showed $58 \%$ deuterium integration at C-1 and 58\% at C-4. No significant deuterium incorporation at any other position was observed.

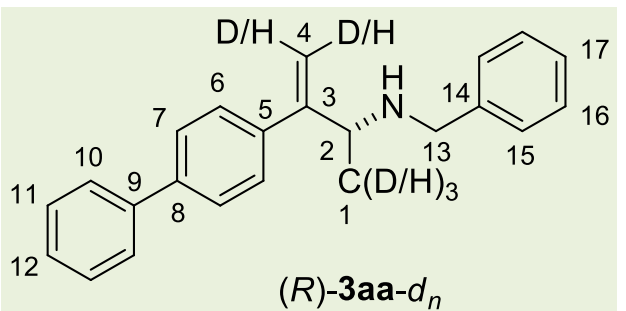

${ }^{1} \mathbf{H}$ NMR $\left(300 \mathrm{MHz}, \mathrm{CDCl}_{3}\right) \delta(\mathrm{ppm})=7.67-7.56(\mathrm{~m}$, $4 \mathrm{H}, \mathrm{H}-A r), 7.53-7.43(\mathrm{~m}, 4 \mathrm{H}, \mathrm{H}-A r), 7.42-7.29(\mathrm{~m}$, $6 \mathrm{H}, \mathrm{H}-A r), 5.42-5.34(\mathrm{~m}, 0.85 \mathrm{H}, \mathrm{H}-4), 3.92\left(\mathrm{~d},{ }^{2} \mathrm{~J}_{\mathrm{HH}}=\right.$ 13.1 Hz, $1 \mathrm{H}, \mathrm{H}-13), 3.87-3.76(\mathrm{~m}, 2 \mathrm{H}, \mathrm{H}-2+\mathrm{H}-13)$, $1.53(\mathrm{~s}, 1 \mathrm{H}, \mathrm{N}-\mathrm{H}), 1.32-1.24(\mathrm{~m}, 1.26 \mathrm{H}, \mathrm{H}-1)$.

${ }^{13} \mathrm{C}\left\{{ }^{1} \mathrm{H}\right\}$ NMR $\left(100 \mathrm{MHz}, \mathrm{CDCl}_{3}\right) \delta(\mathrm{ppm})=152.3-150.9(\mathrm{~m}, \mathrm{C}-3), 141.08(C-A r), 141.05(C-$ Ar), 140.4 (broad, C-Ar), $128.9(\mathrm{CH}-A r), 128.6(\mathrm{CH}-A r), 128.4(\mathrm{CH}-A r), 127.6(\mathrm{CH}-A r), 127.4$ $(\mathrm{CH}-A r), 127.2(\mathrm{CH}-\mathrm{Ar}), 127.1(\mathrm{CH}-\mathrm{Ar}), 127.0(\mathrm{CH}-\mathrm{Ar}), 113.20-112.02\left(\mathrm{C}(\mathrm{H} / \mathrm{D})_{2}-4\right), 57.19-$ $55.93\left(C(\mathrm{H} / \mathrm{D})_{1}-2\right), 51.6\left(\mathrm{CH}_{2}-13\right), 22.0-20.9\left(\mathrm{~m}, \mathrm{C}(\mathrm{H} / \mathrm{D})_{3}-1\right)$.

HRMS $\left(\mathrm{ESI}^{+}\right)$: calculated $[\mathrm{M}+\mathrm{H}]^{+}$for $\mathrm{C}_{17} \mathrm{H}_{19} \mathrm{DN}_{2} \mathrm{O}^{+}$: 315.1942; found: 315.1969; 316.2030; $317.2091 ; 318.211$ (major); 319.2205; 320.2244.

HPLC: $90 \%$ ee, chiral stationary column: OD-H, mobile phase: hexane/iPrOH = 99/1, $1 \mathrm{~mL} / \mathrm{min}, 254 \mathrm{~nm}, 30^{\circ} \mathrm{C}, \mathrm{t}_{\mathrm{R}}($ major $)=6.83 \mathrm{~min}, \mathrm{t}_{\mathrm{R}}($ minor $)=15.14 \mathrm{~min}$. 
<smiles>OC(O)=C(c1ccc(-c2ccccc2)cc1)C(NCc1ccccc1)C(O)(Cl)Cl</smiles>

${ }^{1} \mathrm{H} \mathrm{NMR}\left(300 \mathrm{MHz}, \mathrm{CDCl}_{3}\right)$
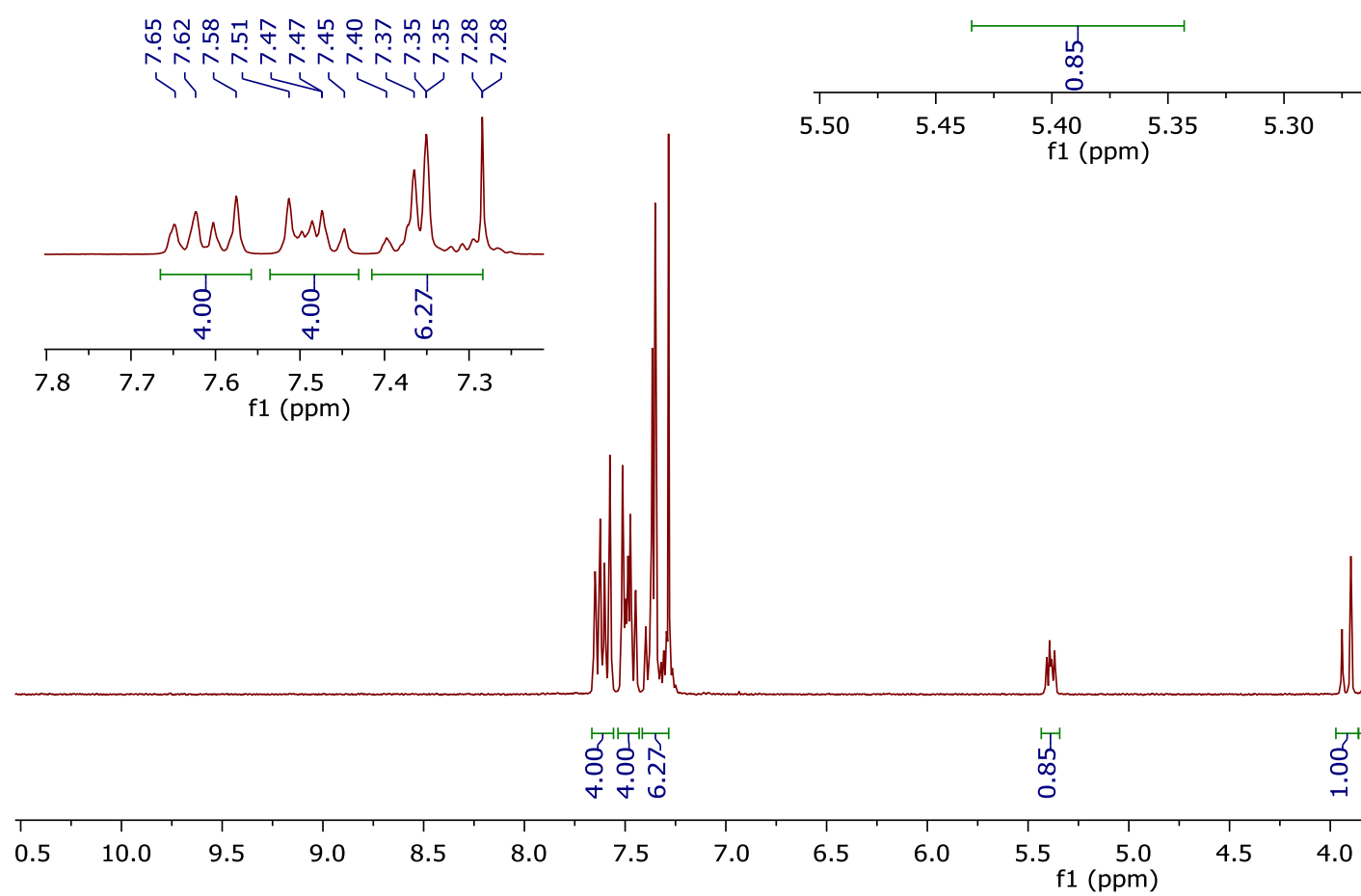

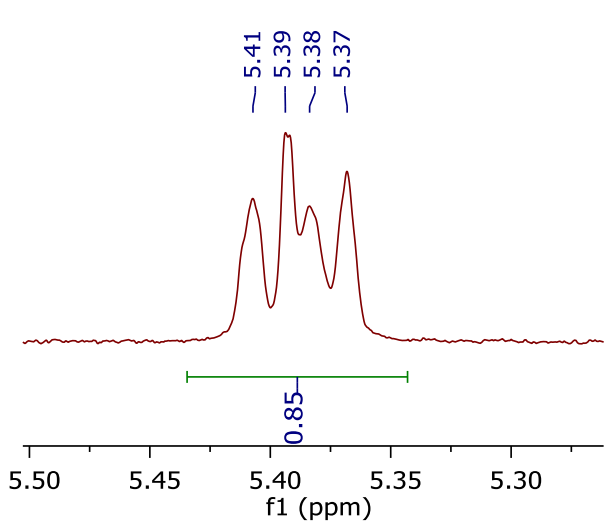

S82
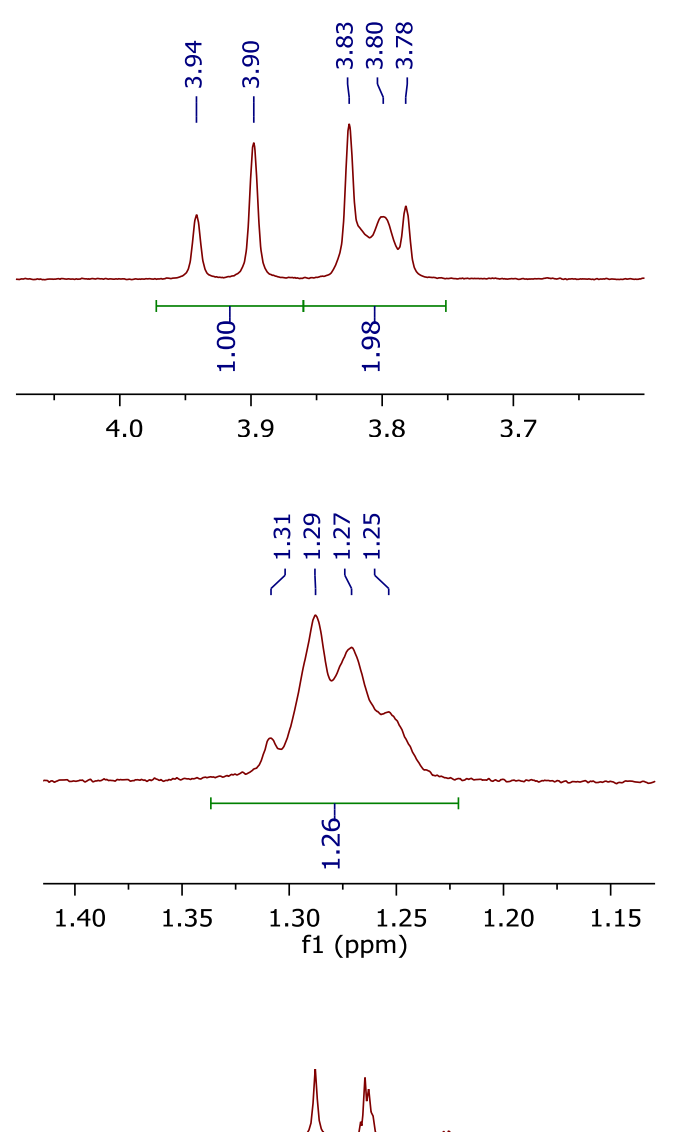

寓

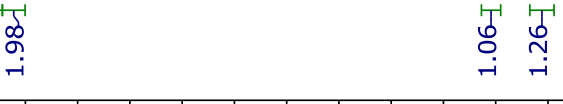


<smiles></smiles>

(R)-3aa- $d_{n}$

${ }^{13} \mathrm{C}\left\{{ }^{1} \mathrm{H}\right] \mathrm{NMR}\left(100 \mathrm{MHz}, \mathrm{CDCl}_{3}\right)$

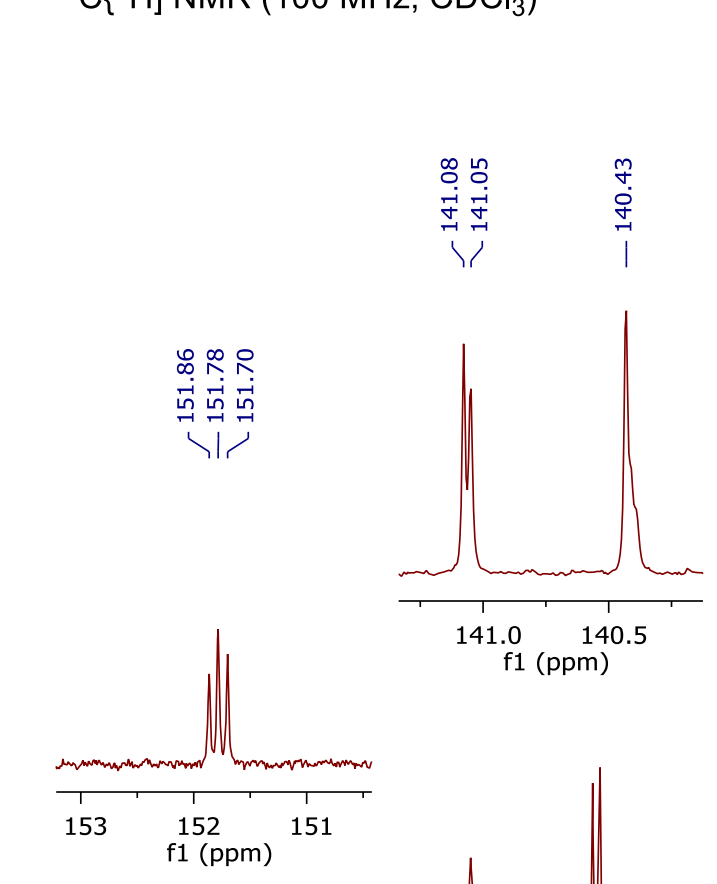

$\infty$ م

군

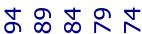

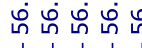

\il?

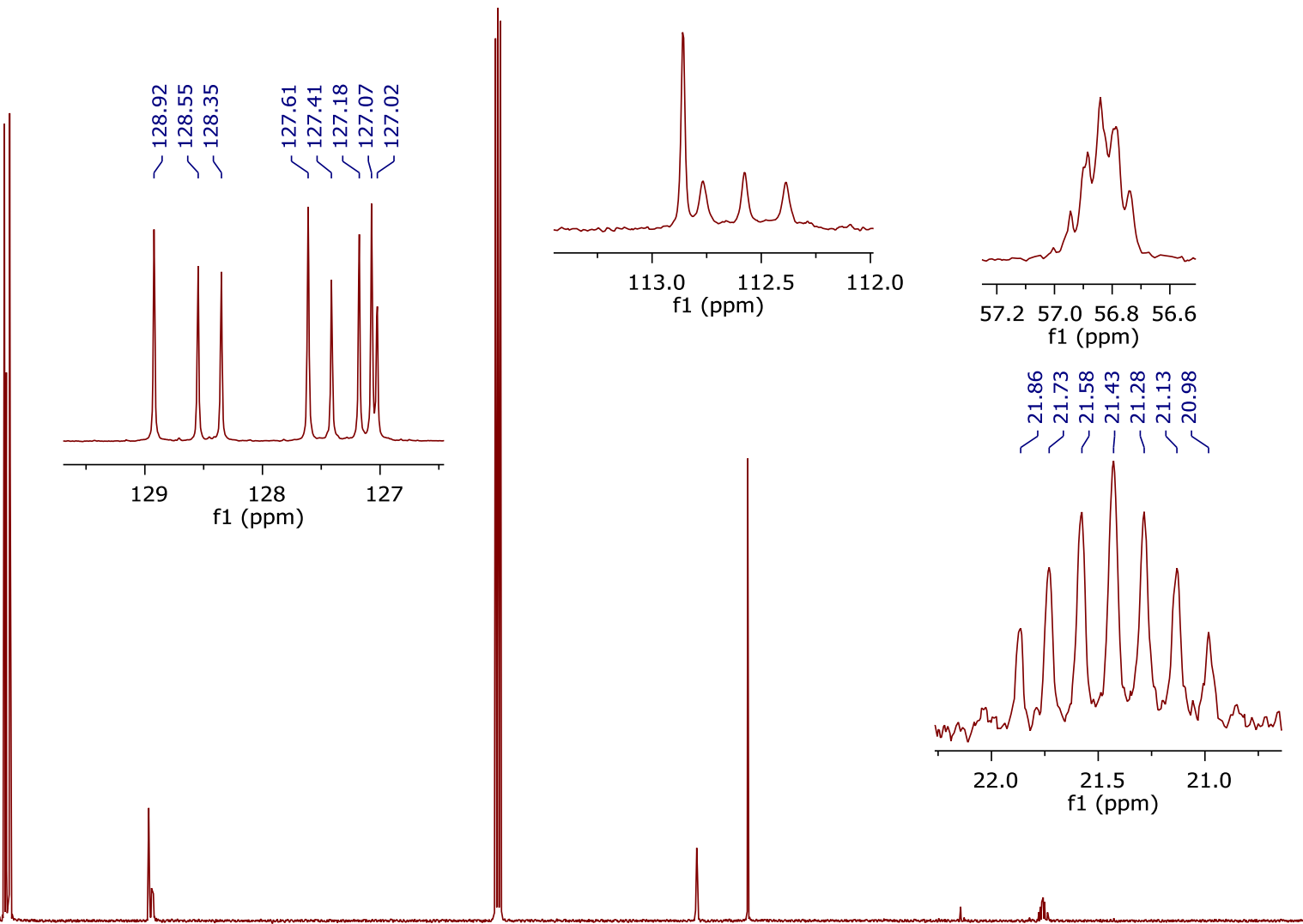

19

180

170

160

150

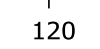

110

$100 \quad 90$ 


\subsection{Post-reaction deuterium incorporation experiment}
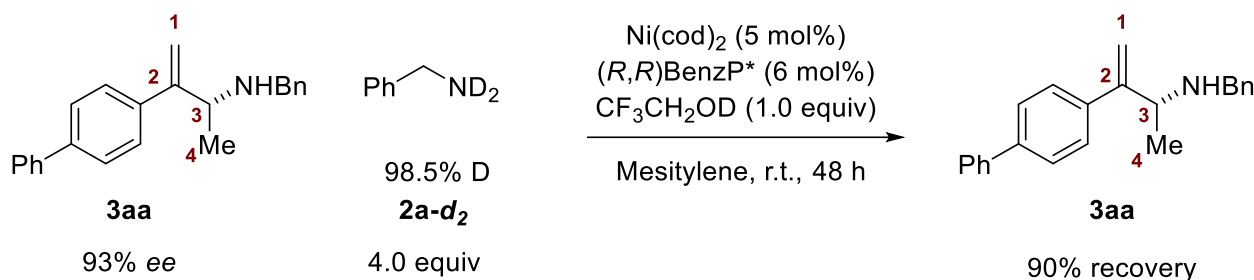

$90 \%$ recovery

$93 \%$ ee

In a $\mathrm{N}_{2}$-filled glovebox, $\mathrm{Ni}(\mathrm{cod})_{2}(1.4 \mathrm{mg}, 0.005 \mathrm{mmol},, 5 \mathrm{~mol} \%)$ and $(R, R)$-BenzP* $\mathrm{L}_{\mathbf{1}}(1.7$ $\mathrm{mg}, 0.006 \mathrm{mmol}, 6 \mathrm{~mol} \%$ ) were weighted in a $5 \mathrm{~mL}$ Schlenk tube, and anhydrous mesitylene $(0.20 \mathrm{~mL}, 0.5 \mathrm{M})$ was added. After stirring at room temperature for $10 \mathrm{~min}$, allylic amine 3aa (31 mg, $0.10 \mathrm{mmol}, 1.0$ equiv, 93\% ee), TFE- $d_{1}(10.0 \mathrm{mg}, 0.10 \mathrm{mmol}, 1.0$ equiv, 99.5\% D) and $\mathrm{Bn}-\mathrm{ND}_{2}(44.0 \mathrm{mg}, 0.40 \mathrm{mmol}, 4.0$ equiv, 98.5\% D) were added sequentially. The tube was sealed, taken out of the glovebox and stirred at room temperature for $48 \mathrm{~h}$. The reaction mixture was filtered over a short pad of silica gel, washed with ethyl acetate $(10 \mathrm{~mL})$ and concentrated under vacuum to afford the crude mixture. Purification by flash chromatography over silica gel (pentane:EtOAc $=4: 1$ ) led to the desired product 3aa as a pale yellow oil (28.0 mg, $90 \%$ recovery, $93 \%$ ee). ${ }^{1} \mathrm{H}$ NMR analysis did not show any trace of deuterium incorporation at any position. 


\subsection{Synthesis of $10-d_{n}$ using TFE-d1 - ${ }^{2} \mathrm{H}$ NMR monitoring}

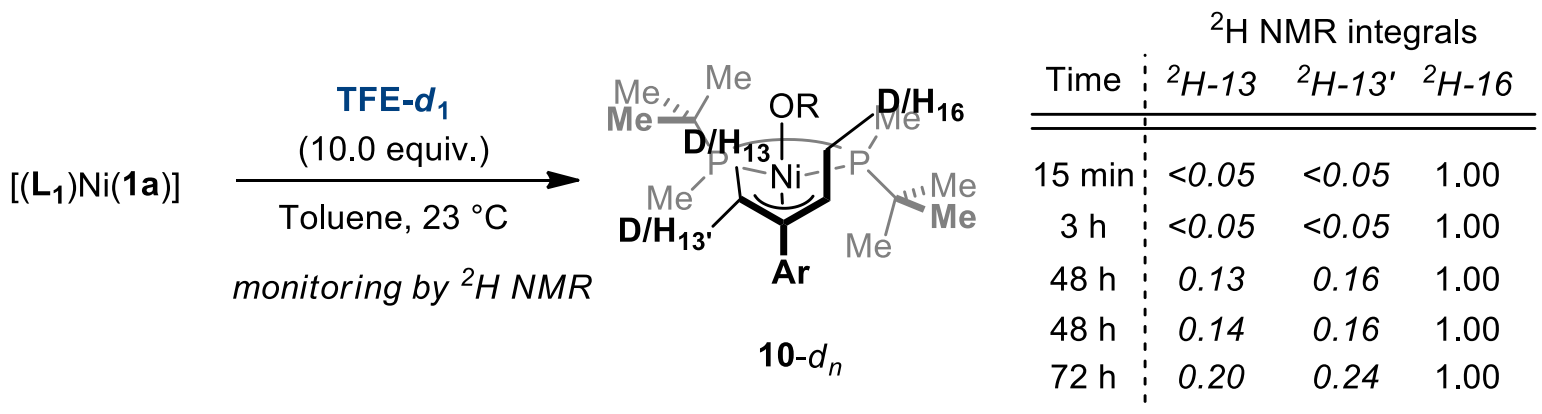

In a $\mathrm{N}_{2}$-filled glovebox, a $2 \mathrm{~mL}$ vial was charged with complex $\left[\left(\mathbf{L}_{\mathbf{1}}\right) \mathrm{Ni}(\mathbf{1 a})\right](\mathbf{9})(5.5 \mathrm{mg}, 0.010$ mmol, 1.0 equiv), followed by toluene $(0.60 \mathrm{~mL})$. To the resulting orange solution was added a $1.0 \mathrm{M}$ solution of $\mathrm{CF}_{3} \mathrm{CH}_{2} \mathrm{OD}$ in toluene $(0.10 \mathrm{~mL}, 0.10 \mathrm{mmol}, 10.0$ equiv). The color of the solution changed immediately to pale orange/yellow. $0.40 \mathrm{~mL}$ of this solution was introduced in a J-Young tube. ${ }^{2} \mathrm{H}$ NMR monitoring showed very rapid and selective deuterium incorporation at $\mathrm{C}-4(\mathrm{D} / \mathrm{H}-16)$, and very slow deuterium incorporation at $\mathrm{C}-1(\mathrm{D} / \mathrm{H}-13$ and $\left.\mathrm{D} / \mathrm{H}-13^{\prime}\right)$.

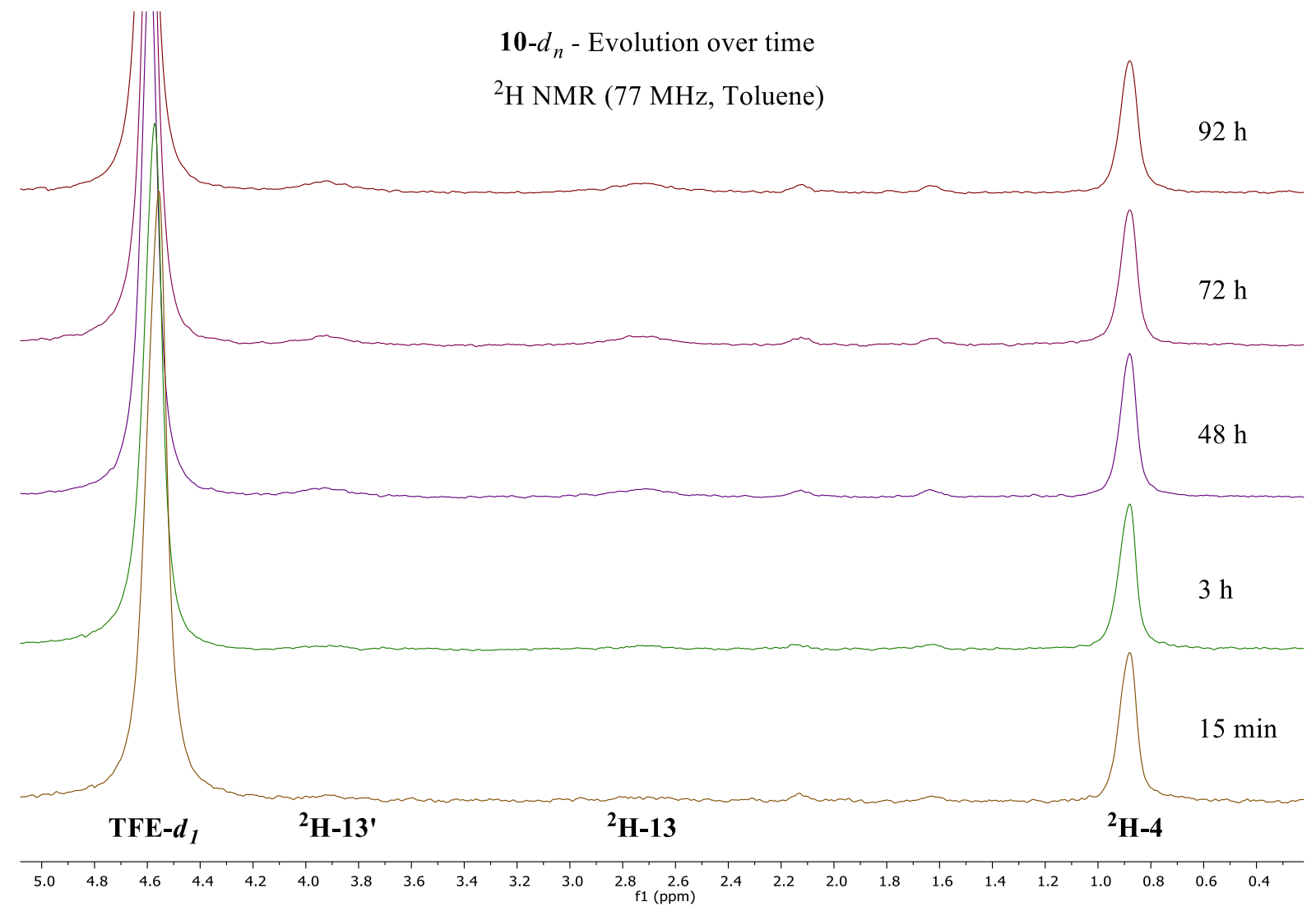




\subsection{Synthesis of $10-d_{n}$ using TFE- $d_{1}$ and $\mathrm{BnND}_{2^{-}}{ }^{1} \mathrm{H}$ NMR monitoring}

\begin{tabular}{c:ccc} 
Time & ${ }^{1} H-13$ & ${ }^{1} H-13$ & ${ }^{1} H-15$ \\
\hline $20 \mathrm{~min}$ & 1.03 & 0.84 & 1.00 \\
$2 \mathrm{~h}$ & 0.65 & 0.45 & 1.00 \\
$5 \mathrm{~h}$ & 0.36 & 0.24 & 0.95 \\
$10 \mathrm{~h}$ & 0.35 & 0.22 & 1.02 \\
$18 \mathrm{~h}$ & 0.38 & 0.24 & 1.01
\end{tabular}

a) ${ }^{\mathrm{B}} \mathrm{Bu}$ signals from the ligand backbone were used as internal reference.

In a $\mathrm{N}_{2}$-filled glovebox, a $2 \mathrm{~mL}$ vial was charged with complex [( $\left.\left.\mathbf{L}_{\mathbf{1}}\right) \mathrm{Ni}(\mathbf{1 a})\right](\mathbf{9})(16.6 \mathrm{mg}, 0.030$ $\mathrm{mmol}, 1.0$ equiv), followed by toluene- $d_{8}(1.0 \mathrm{~mL})$. To the resulting orange solution was added neat $\mathrm{CF}_{3} \mathrm{CH}_{2} \mathrm{OD}$ (31.0 mg, $0.303 \mathrm{mmol}, 10.0$ equiv). The color of the solution changed immediately to pale orange/yellow. $0.40 \mathrm{~mL}$ of this solution was introduced in a J-Young tube, followed by $\mathrm{BnND}_{2}$ (13.0 $\mathrm{mg}, 0.120 \mathrm{mmol}, 10.0$ equiv). Deuterium integration at $\mathrm{C}-1$ $\left(\mathrm{D} / \mathrm{H}-13\right.$ and $\left.\mathrm{D} / \mathrm{H}-13^{\prime}\right)$ and $\mathrm{C}-3(\mathrm{D} / \mathrm{H}-15)$ was then monitored by ${ }^{1} \mathrm{H}$ NMR. Deuterium integration at C-4 (D/H-16) could not be accomplished due to signals overlap.

10- $d_{n}-$ Evolution over time

${ }^{1} \mathrm{H}$ NMR (400 MHz, Tol- $d_{8}$ )

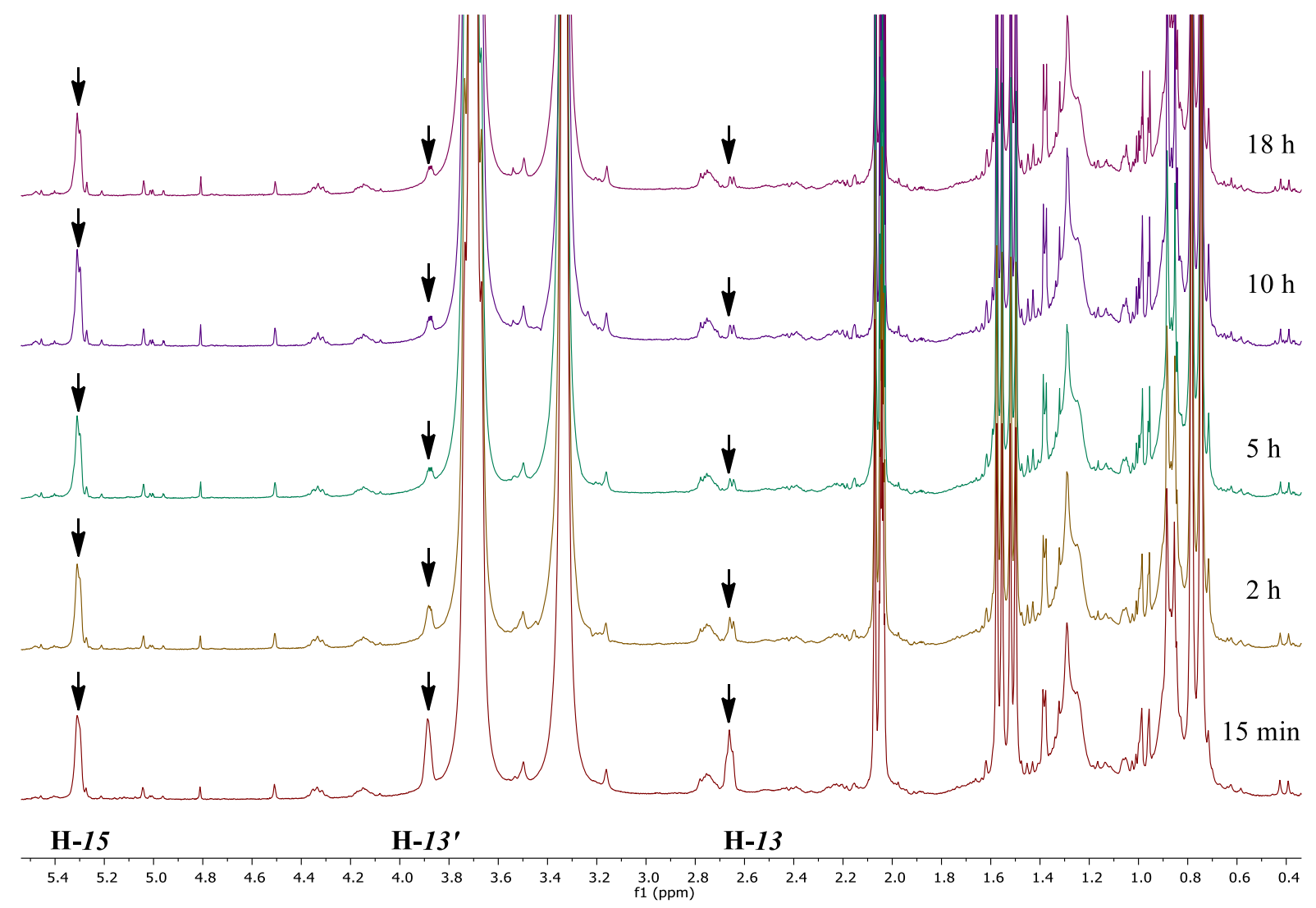




\section{Kinetic studies}

\section{General:}

In situ ${ }^{1} \mathrm{H}$ NMR monitoring was carried out in J-Young NMR tube using a AMX-500 Bruker Avance spectrometer. The reaction was monitored by following the consumption of substrate 1a using the allylic proton at C-3. Spectra were collected at $298 \mathrm{~K}$ every 1 or 2 hours for the entirety of the experiment ( 5 scans, 10 seconds T1 relaxation delay). The data were treated using the methods for variable time normalization analysis. ${ }^{3}$ Given the absence of by-products containing $\mathrm{BnNH}_{2}$, the concentration of $\mathrm{BnNH}_{2}$ [2a $]_{\mathrm{t}}$ for each time $t$ was calculated using the following formula: $[\mathbf{2 a}]_{t}=[\mathbf{2 a}]_{t=0}-\left([\mathbf{1} \mathbf{a}]_{t=0}-[\mathbf{1} \mathbf{a}]_{t}\right)$.

\section{Standard reaction procedure:}

In an $\mathrm{N}_{2}$-filled glove-box, a solution of $\mathrm{Ni}(\operatorname{cod})_{2}(4.1 \mathrm{mg}, 0.0150 \mathrm{mmol})$ and $(R, R) B e n z P^{*} L_{1}$ $(5.1 \mathrm{mg}, 0.0181 \mathrm{mmol})$ in toluene- $d_{8}(0.60 \mathrm{~mL})$ was prepared in a $2 \mathrm{~mL}$ vial. After stirring for 5 min, substrate 1a $(61.9 \mathrm{mg}, 0.300 \mathrm{mmol})$ was added, stirring for continued for $30 \mathrm{~min}$, and $\mathrm{BnNH}_{2}$ 2a (128.6 mg, $131 \mu \mathrm{L}, 1.200 \mathrm{mmol}$ ) was added. In a J-Young tube equipped with a sealed glass capillary containing an internal standard solution ( $p$-methylanisole $\sim 0.10 \mathrm{M}$; $\mathrm{PPh}_{3} \sim 0.01 \mathrm{M}$; solvent: toluene- $\left.d_{8}\right)$ was introduced $0.49 \mathrm{~mL}$ of the resulting stock solution $\left(\left[\mathrm{Ni}(\mathrm{cod})_{2}\right]=0.0205 \mathrm{M} ;\left[\mathbf{L}_{1}\right]=0.0248 \mathrm{M} ;[1 \mathrm{a}]=0.410 \mathrm{M} ;[2 \mathrm{a}]=1.641 \mathrm{M}\right) .{ }^{1} \mathrm{H}$ NMR of this solution was acquired, allowing for calibration of the ${ }^{1} \mathrm{H}$ internal standard peaks (time $=0 \mathrm{~s}$ ). The J-Young tube was then brought back into an $\mathrm{N}_{2}$-filled glovebox, catalysis was initiated by addition of trifluoroethanol $(20.0 \mathrm{mg}, 0.200 \mathrm{mmol}, 1.0$ equiv, [TFE] $=0.410 \mathrm{M})$, and the reaction mixture was monitored over time by ${ }^{1} \mathrm{H}$ NMR.

\section{Same excess}

This reaction was carried out according to the standard reaction procedure, with the following concentrations: $\left[\mathrm{Ni}(\operatorname{cod})_{2}\right]=0.0205 \mathrm{M} ;\left[\mathbf{L}_{1}\right]=0.0248 \mathrm{M} ;[1 \mathrm{a}]=0.250 \mathrm{M} ;[\mathbf{2 a}]=1.500 \mathrm{M} ;[\mathrm{TFE}]$ $=0.410 \mathrm{M})$. 

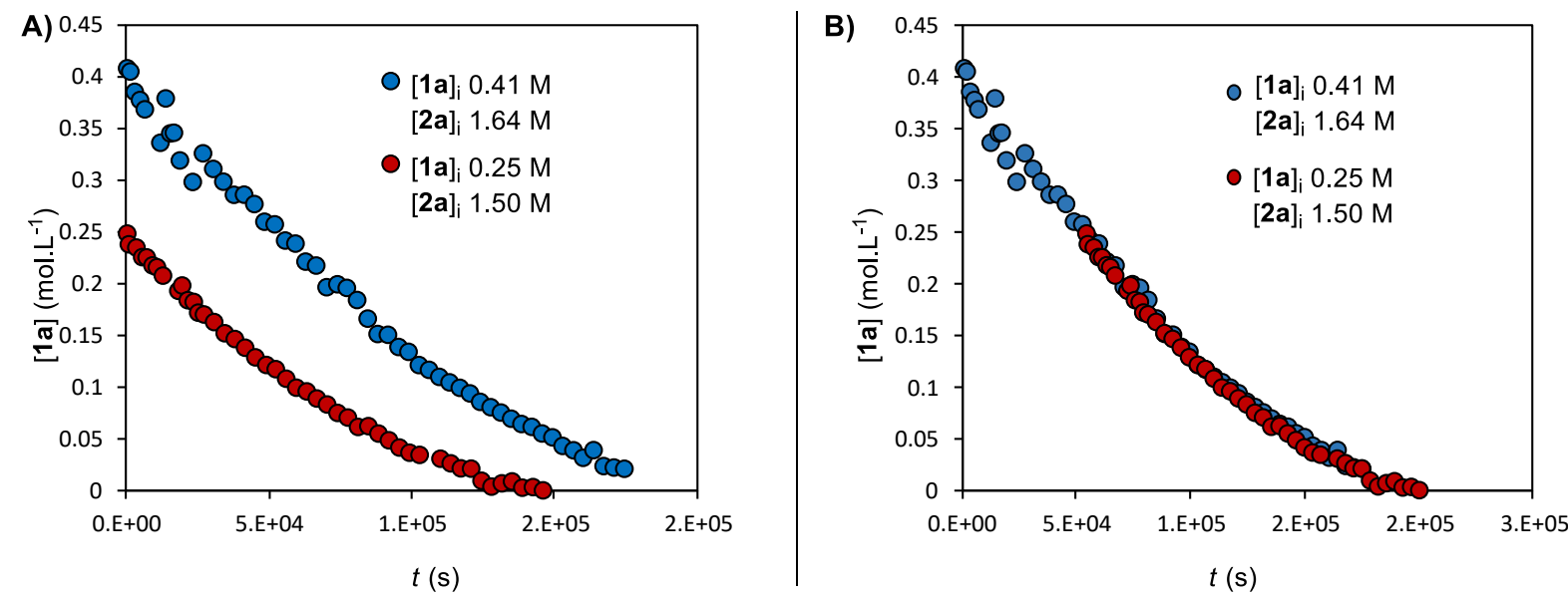

Figure S3. A) Reaction time course for "standard conditions" (blue points) and "same excess" experiment (red points). B) Reaction time course for standard and same excess experiments after time adjustment of the "same excess" experiment (red points; $\Delta t=+53550$ s).

\section{Order in catalyst}

This reaction was carried out according to the standard reaction procedure, with the following concentrations: $\left[\mathrm{Ni}(\mathrm{cod})_{2}\right]=0.0410 \mathrm{M} ;\left[\mathrm{L}_{1}\right]=0.0496 \mathrm{M} ;[1 \mathrm{a}]=0.410 \mathrm{M} ;[2 \mathrm{a}]=1.641 \mathrm{M} ;[\mathrm{TFE}]$ $=0.410 \mathrm{M})$.
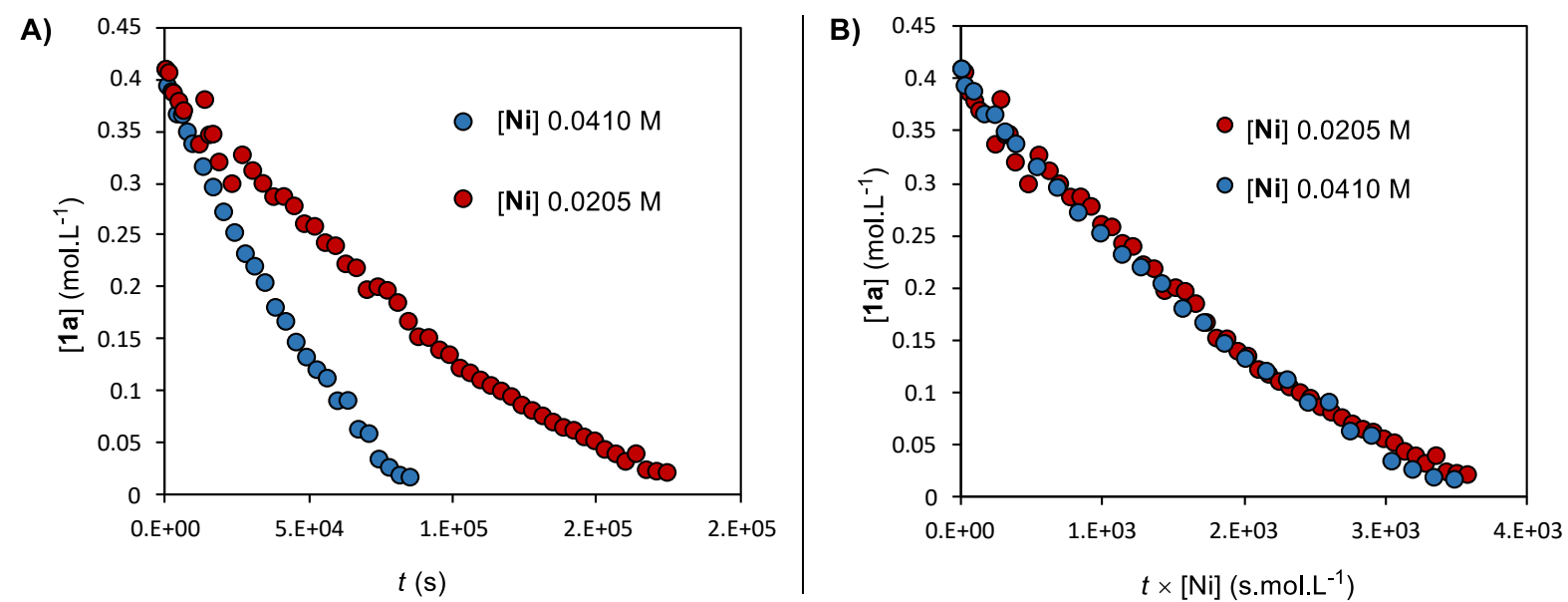

Figure S-4. A) Reaction time course for "standard conditions" (red points) and "Order in catalyst" experiment (blue points). B) Reaction time course for standard and "Order in catalyst" experiments after multiplication of the original time axis by the catalyst initial concentration (time $\left.x[\mathrm{Ni}(\mathrm{cod})]_{2}\right)$. 


\section{Order in diene 1a}

This reaction was carried out according to the standard reaction procedure, with the following concentrations: $\left[\mathrm{Ni}(\operatorname{cod})_{2}\right]=0.0205 \mathrm{M} ;\left[\mathrm{L}_{1}\right]=0.0248 \mathrm{M} ;[1 \mathrm{a}]=0.615 \mathrm{M} ;[2 \mathrm{a}]=1.641 \mathrm{M} ;[\mathrm{TFE}]$ $=0.410 \mathrm{M})$.
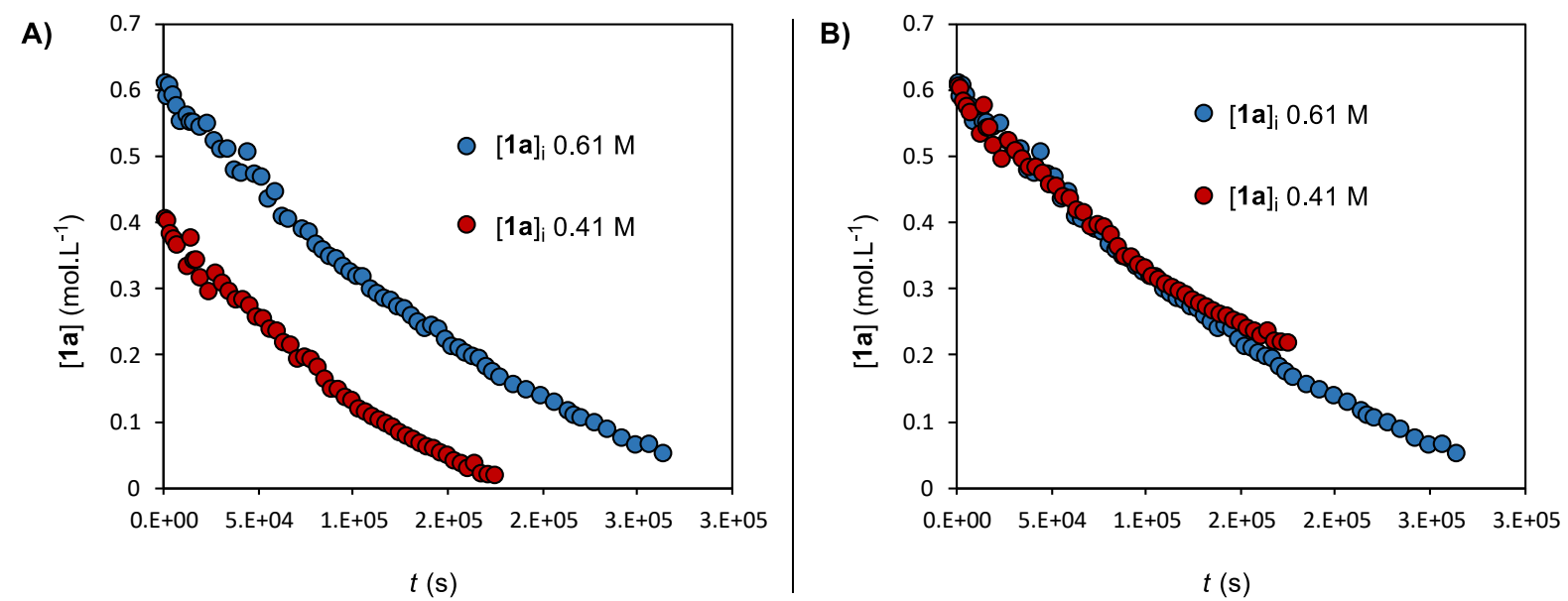

Figure S-5. A) Reaction time course for "standard conditions" (red points) and "Order in diene" experiment (blue points). B) Reaction time course for standard and "Order in diene" experiments after upward concentration shift of the "standard conditions" points (red points: $[1 \mathrm{a}]+0.205 \mathrm{M})$.

\section{Order in TFE}

These reactions were carried out according to the standard reaction procedure, with the following concentrations:

- Red points: $\left[\mathrm{Ni}(\operatorname{cod})_{2}\right]=0.0205 \mathrm{M} ;\left[\mathrm{L}_{1}\right]=0.0248 \mathrm{M} ;[1 \mathrm{a}]=0.410 \mathrm{M} ;[2 \mathrm{a}]=1.641 \mathrm{M} ;[\mathrm{TFE}]=$ $0.290 \mathrm{M})$.

- Yellow points: $\left[\mathrm{Ni}(\mathrm{cod})_{2}\right]=0.0205 \mathrm{M} ;\left[\mathrm{L}_{1}\right]=0.0248 \mathrm{M} ;[1 \mathrm{a}]=0.410 \mathrm{M} ;[2 \mathrm{a}]=1.641 \mathrm{M} ;[\mathrm{TFE}]$ $=0.530 \mathrm{M})$. 
A)

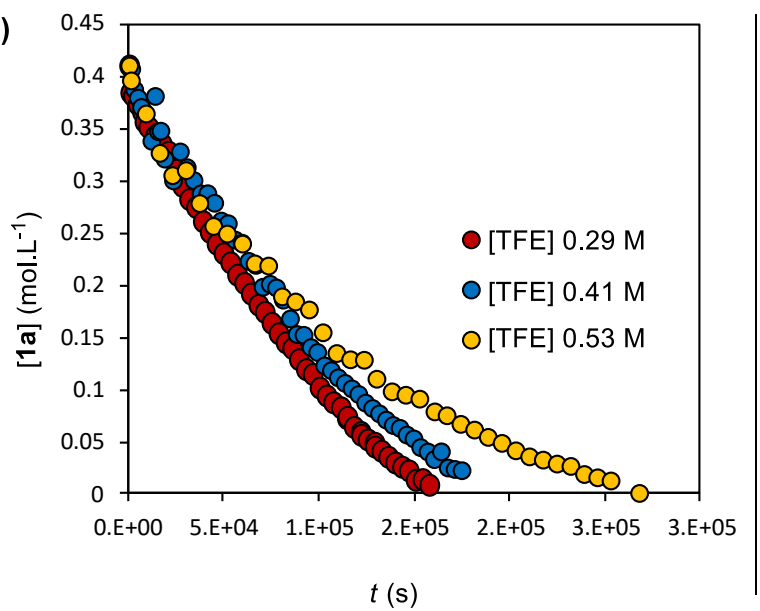

B)

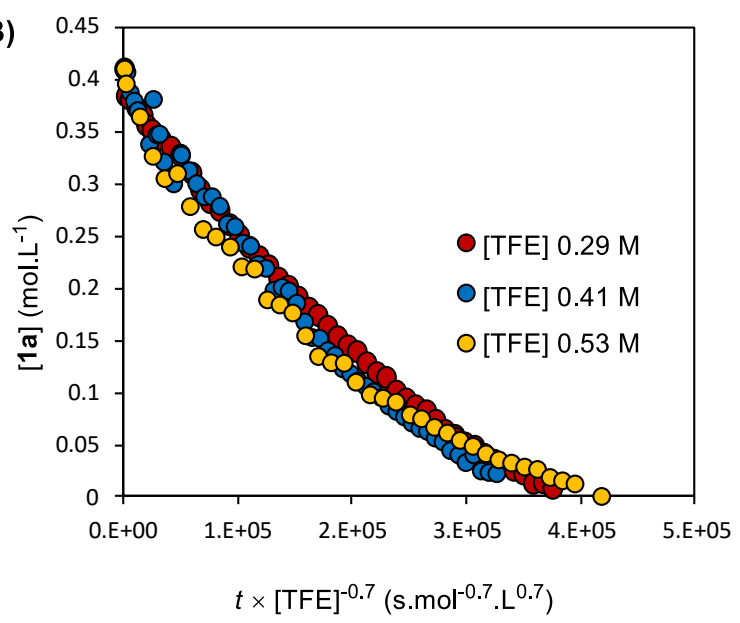

Figure S-6. A) Reaction time course for "standard conditions" (red points) and "Order in TFE" experiments (blue and yellow points). B) Reaction time course for standard and " Order in TFE " experiments after multiplication of the original time axis by [TFE] $]^{-0.7}$ (time $x[\text { TFE }]^{-0.7}$ ).

\section{Order in $\mathrm{BnNH}_{2} 2 \mathrm{a}$}

These reactions were carried out according to the standard reaction procedure, with the following concentrations:

- Red points: $\left[\mathrm{Ni}(\operatorname{cod})_{2}\right]=0.0205 \mathrm{M} ;\left[\mathrm{L}_{1}\right]=0.0248 \mathrm{M} ;[1 \mathrm{a}]=0.410 \mathrm{M} ;[2 \mathrm{a}]=1.290 \mathrm{M} ;[\mathrm{TFE}]=$ $0.410 \mathrm{M})$.

- Yellow points: $\left[\mathrm{Ni}(\operatorname{cod})_{2}\right]=0.0205 \mathrm{M} ;\left[\mathrm{L}_{1}\right]=0.0248 \mathrm{M} ;[1 \mathrm{a}]=0.410 \mathrm{M} ;[2 \mathrm{a}]=1.961 \mathrm{M} ;[\mathrm{TFE}]$ $0.410 \mathrm{M})$. 
A)

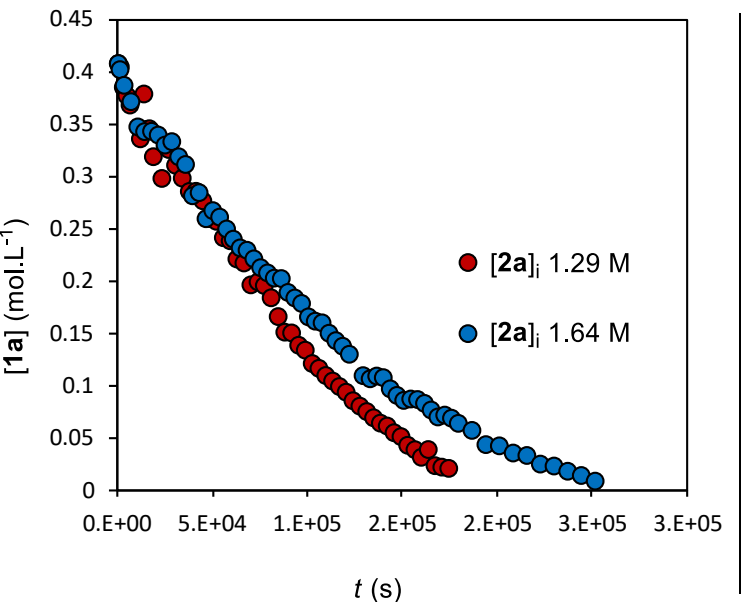

C)

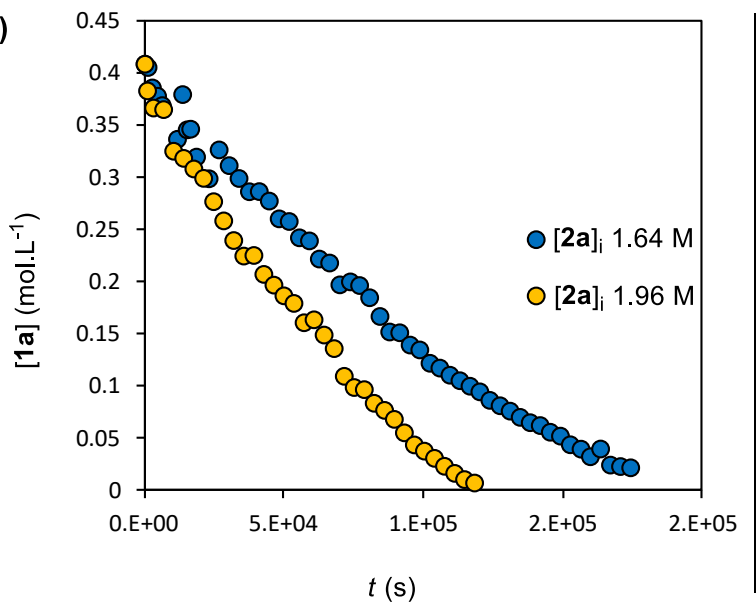

B)

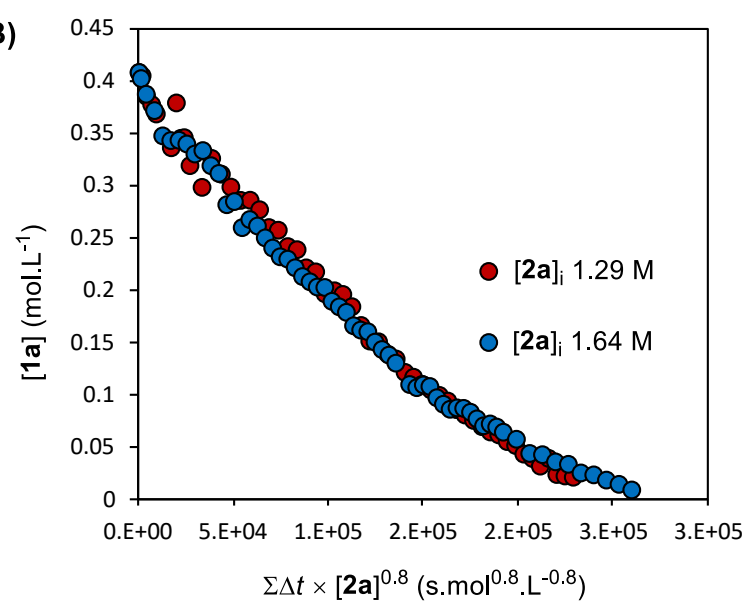

D)

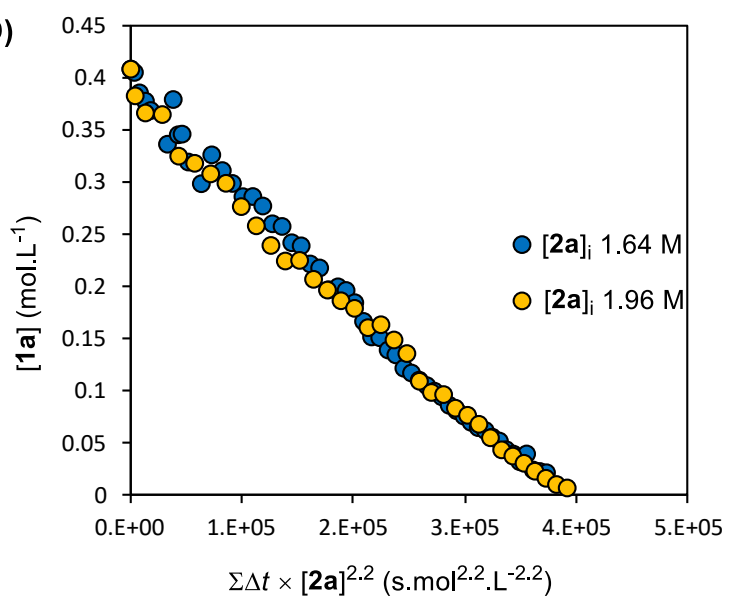

Figure S-7. A) and C) Reaction time course for standard conditions (blue points) and "Order in $\mathrm{BnNH}_{2}$ 2a" experiments (red and yellow points). B) Reaction time course for standard and "Order in $\mathrm{BnNH}_{2}(1.29 \mathrm{M})$ " experiments after variable time normalization of the original time axis using $t_{n}=\sum_{i=1}^{n}\left(t_{\mathrm{i}}-t_{\mathrm{i}-1}\right)\left(\frac{[2 a]_{\mathrm{i}}+[2 a]_{\mathrm{i}-1}}{2}\right){ }^{0.8}$. D) Reaction time course for standard and "Order in $\mathrm{BnNH}_{2} \mathbf{2 a}(1.96 \mathrm{M}) "$ experiments after variable time normalization of the original time axis using $\mathrm{t}_{\mathrm{n}}=\sum_{i=1}^{n}\left(t_{\mathrm{i}}-t_{\mathrm{i}-1}\right)\left(\frac{[2 a]_{\mathrm{i}}+[2 a]_{\mathrm{i}-1}}{2}\right)^{2.2}$. 


\section{1. $\mathrm{BnNH}_{2}$ NMR titration by TFE}

In an $\mathrm{N}_{2}$-filled glove-box, $\mathrm{BnNH}_{2}(86 \mathrm{mg}, 88 \mu \mathrm{L}, 0.80 \mathrm{mmol})$ and toluene- $d_{8}(0.40 \mathrm{~mL})$ were charged in a J-Young NMR tube equipped with a sealed glass capillary containing an internal standard solution ( $p$-methylanisole $\sim 0.010 \mathrm{M}$; solvent: toluene- $d_{8}$ ). ${ }^{1} \mathrm{H}$ NMR of this solution was recorded, the J-Young tube was brought back into the glovebox. Increasing amounts of trifluoroethanol were then added sequentially, and ${ }^{1} \mathrm{H}$ NMR spectra were recorded between each point (Figure S-8).

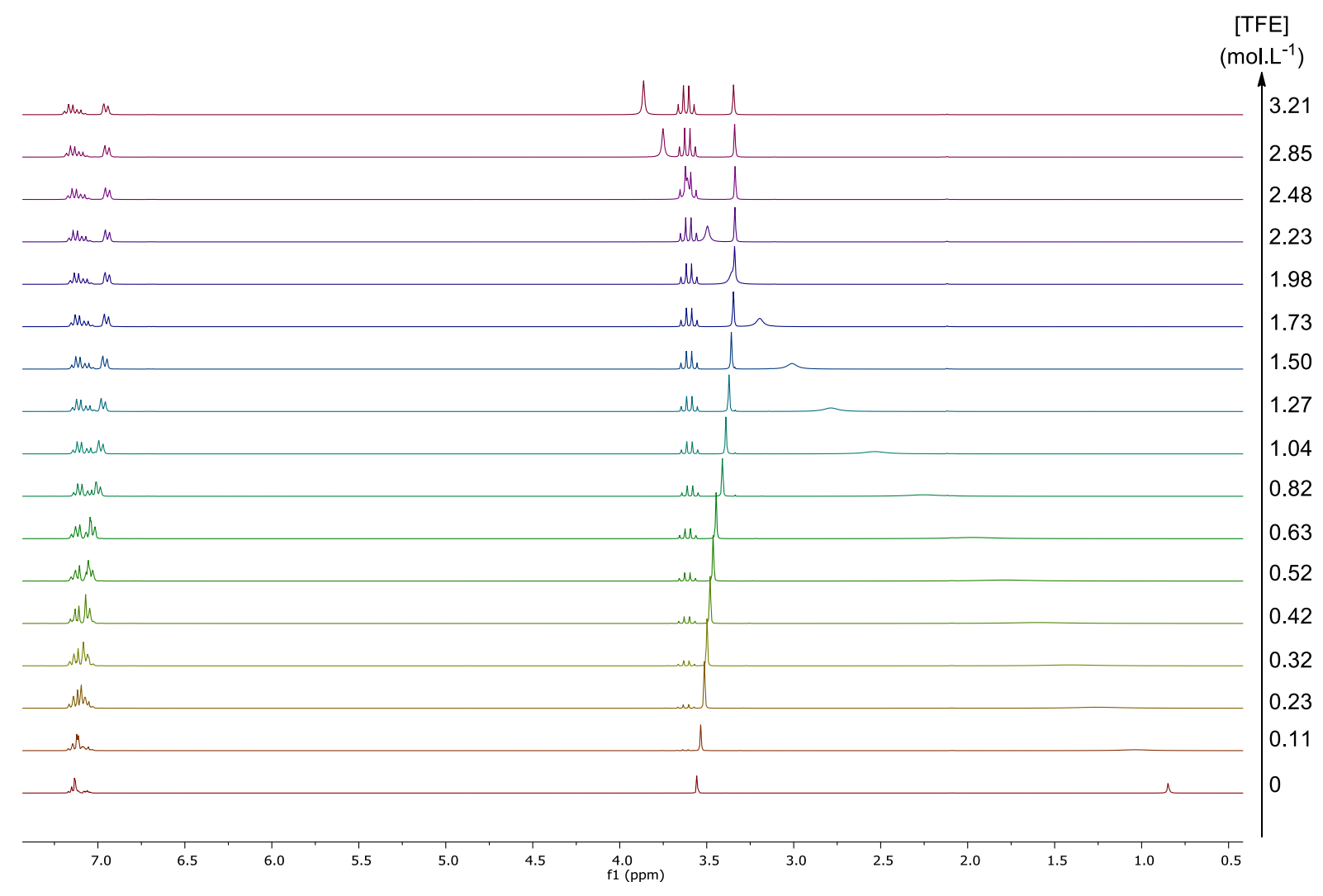

Figure S-8. ${ }^{1} \mathrm{H}$ NMR of $\left[\mathrm{BnNH}_{2}: \mathrm{TFE}\right]$ solutions in toluene- $d_{8}$.

The $\Delta \delta{ }^{1} \mathrm{H}$ NMR shift of the $\mathrm{BnNH}_{2}$ benzylic protons for each point compared to the initial $1.64 \mathrm{M} \mathrm{BnNH}_{2}$ solution was then plotted against the TFE concentration (Figure S-9).

These data were fitted to the 1:1, 2:1 or 1:2 binding models proposed by Thodarson et al.. ${ }^{4}$ Good Root Means Square errors were obtained for the 1:2 and 2:1 $\mathrm{BnNH}_{2}$ :TFE models (RMS $=0.0028$ and 0.0024 respectively). Although it is not possible to unequivocally determine which one of these fits is better, a 1:2 fit - where a $\mathrm{BnNH}_{2}: \mathrm{TFE}$ dimer would form first prior to binding of a second equivalent of TFE to the lone pair of the oxygen of the first molecule of alcohol - seems more likely. Indeed, the signal of the benzylic hydrogens are no longer significantly shifting once the concentration in TFE and $\mathrm{BnNH}_{2}$ are equals. 
Subsequently, the signal of the exchangeable protons of benzylamine sharpen until a $1: 2$ stoichiometry is reached.

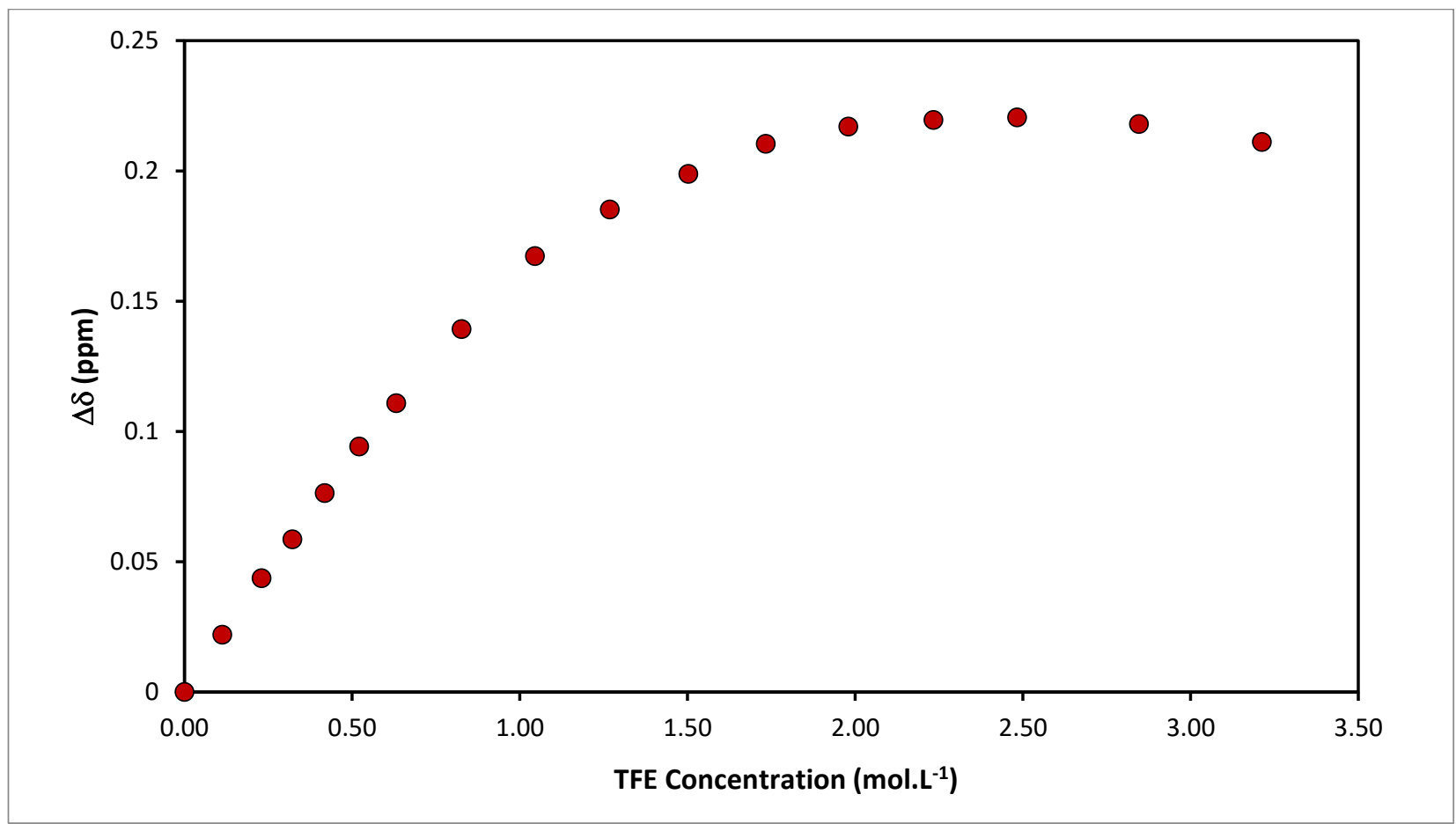

Figure S-9. ${ }^{1} \mathrm{H}$ NMR titration of Benzylic amine by $\mathrm{CF}_{3} \mathrm{CH}_{2} \mathrm{OH}$. 
12. NMR Spectra of organic compounds 
$\checkmark$

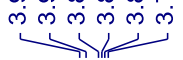

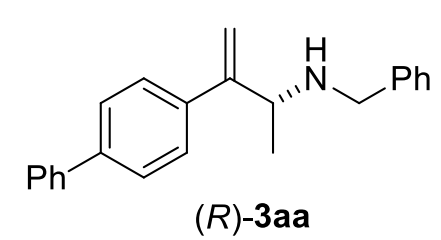

${ }^{1} \mathrm{H} \mathrm{NMR}\left(400 \mathrm{MHz}, \mathrm{CDCl}_{3}\right)$
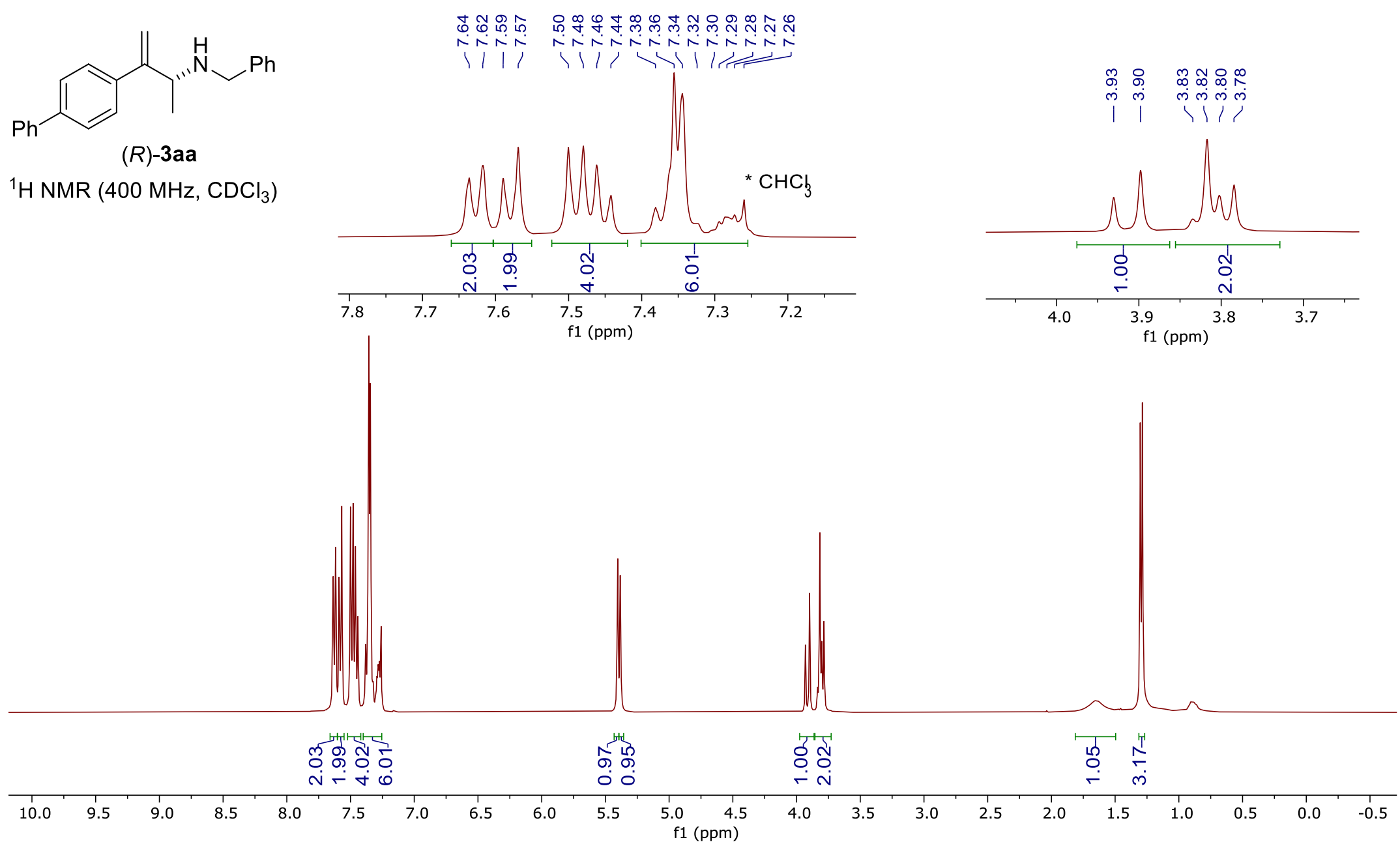


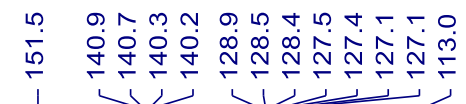

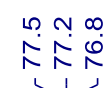

in

$\stackrel{\infty}{i}$<smiles>C=C(c1ccc(-c2ccccc2)cc1)[C@H](C)NCc1ccccc1</smiles>

(R)-3aa

${ }^{13} \mathrm{C}\left\{{ }^{1} \mathrm{H}\right\} \mathrm{NMR}\left(100 \mathrm{MHz}, \mathrm{CDCl}_{3}\right)$
${ }^{*} \mathrm{CDCl}_{3}$

万.

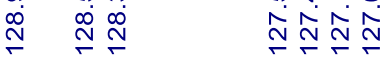

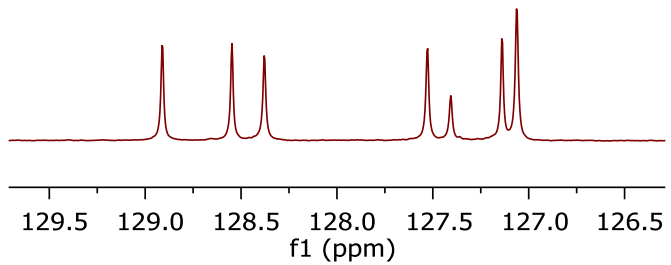

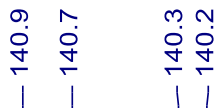

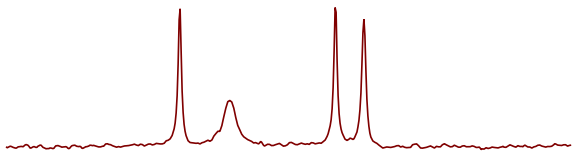

41.5

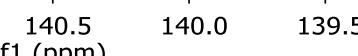

f1 (ppm)

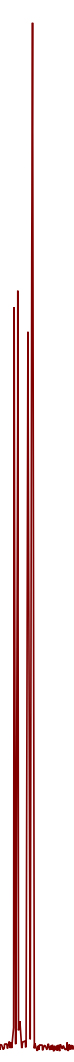

210

$200 \quad 190$

$190 \quad 180$

160

$150 \quad 140$

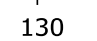

120

100

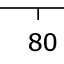

$70 \quad 60$

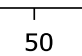

$40 \quad 30$

20

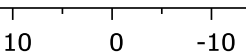




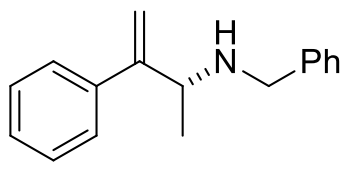

(R)-3ba

${ }^{1} \mathrm{H}$ NMR $\left(400 \mathrm{MHz}, \mathrm{CDCl}_{3}\right)$
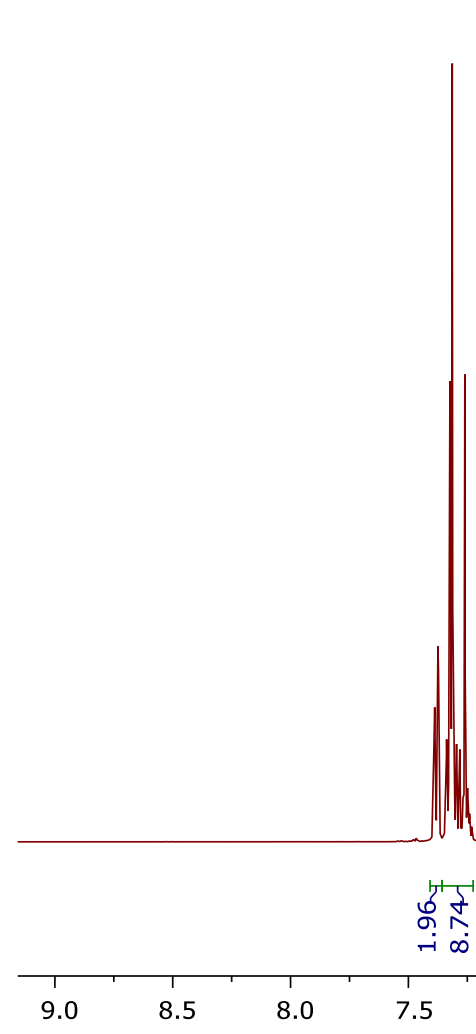

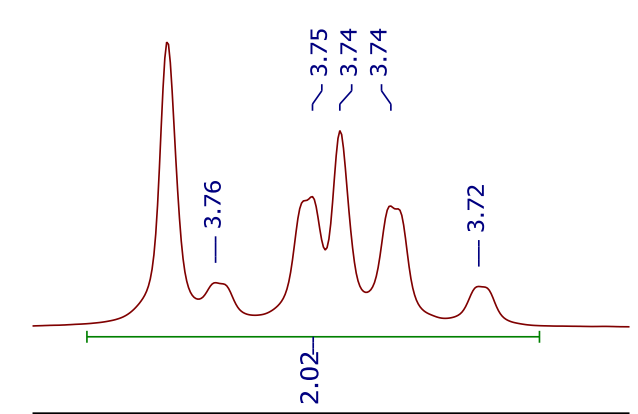

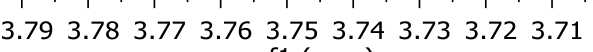

$$
\text { f1 (ppm) }
$$

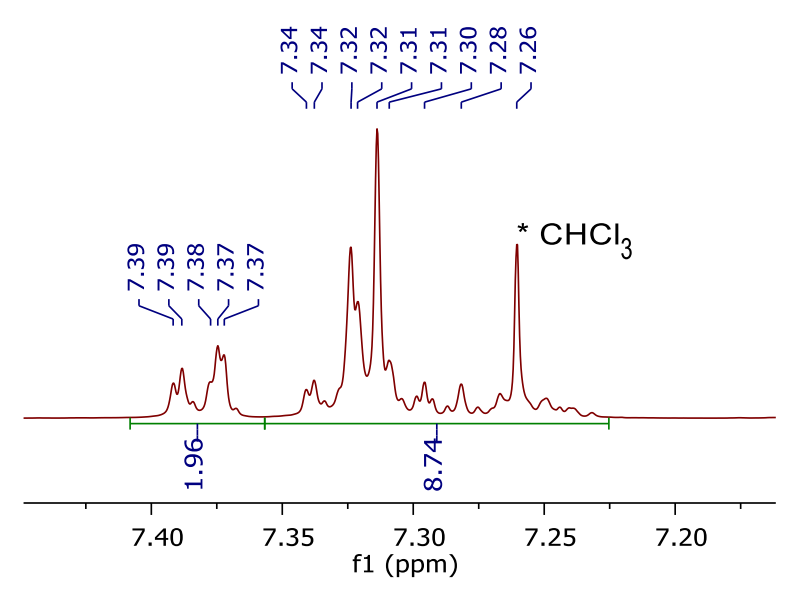

$$
\text { ren }
$$

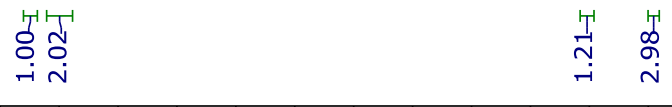



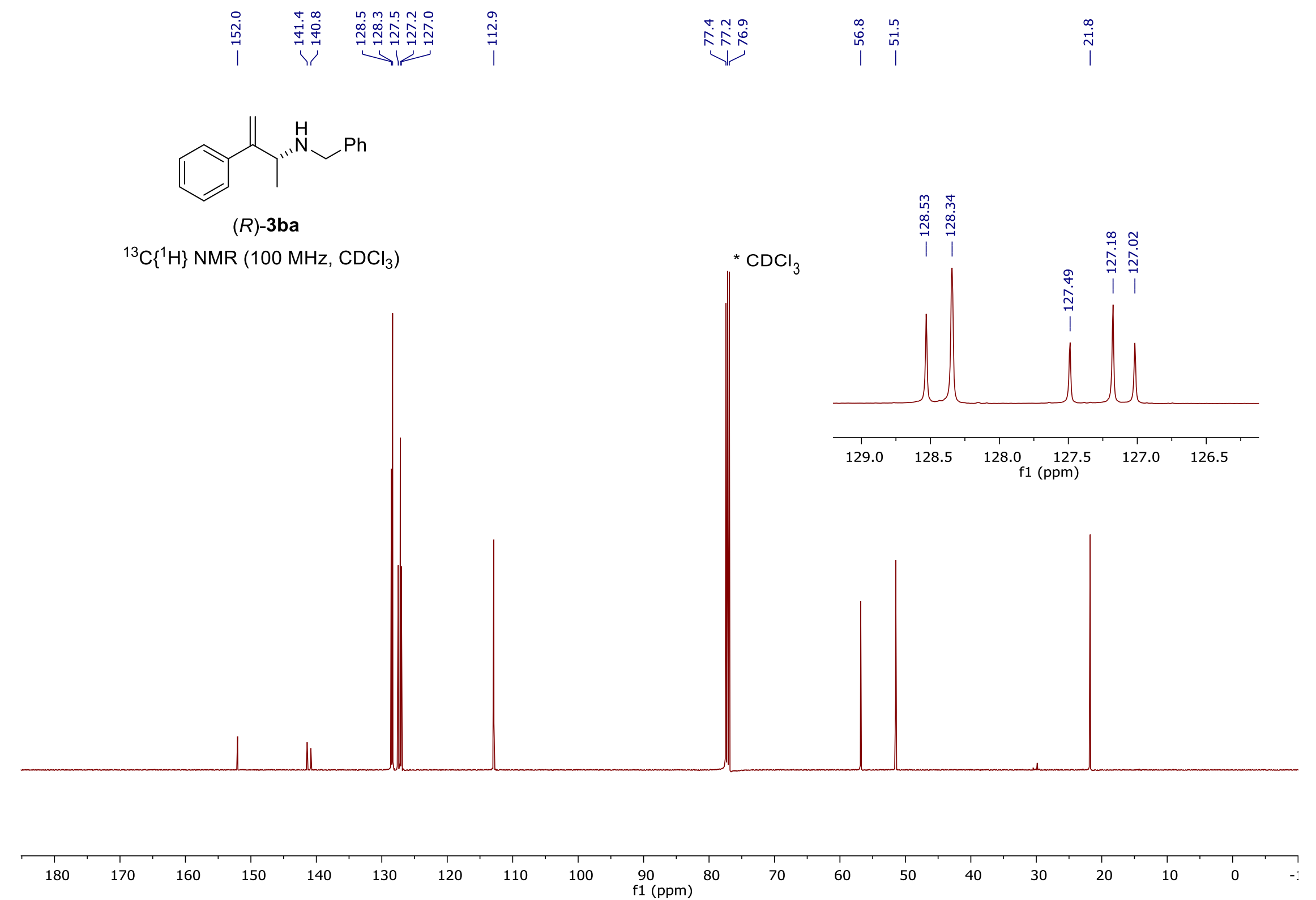


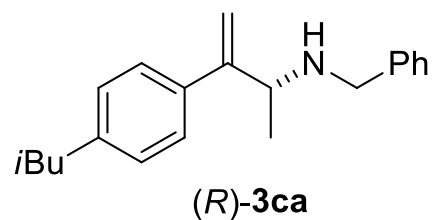

${ }^{1} \mathrm{H}$ NMR $\left(400 \mathrm{MHz}, \mathrm{CDCl}_{3}\right)$

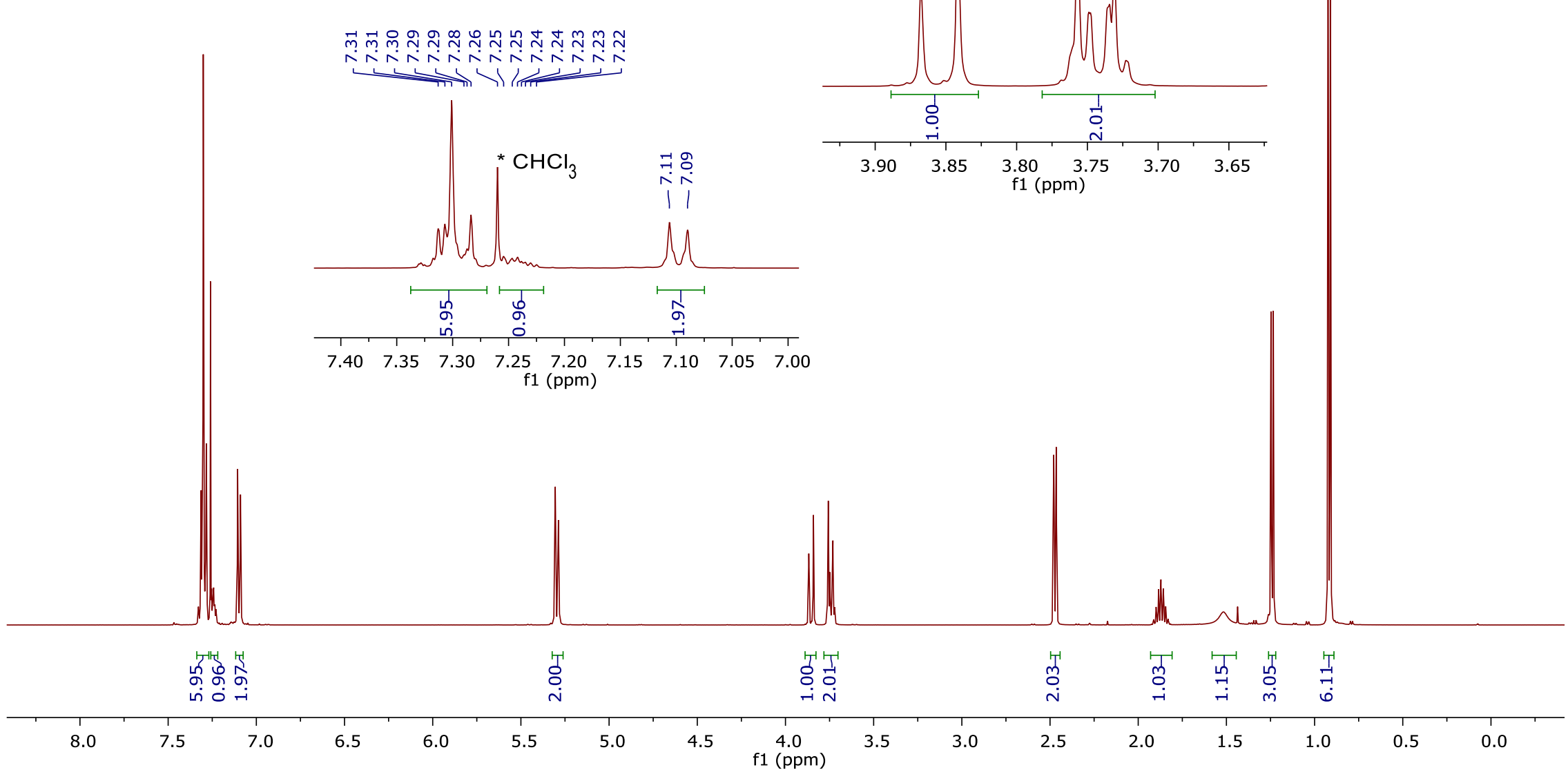




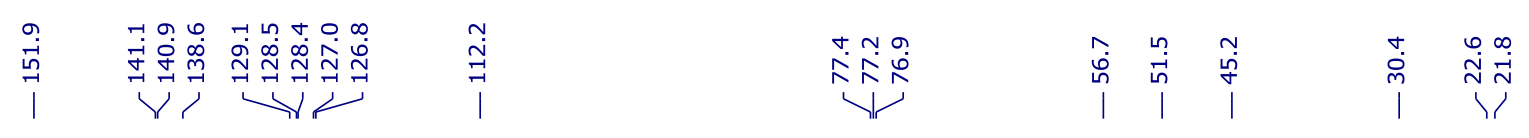

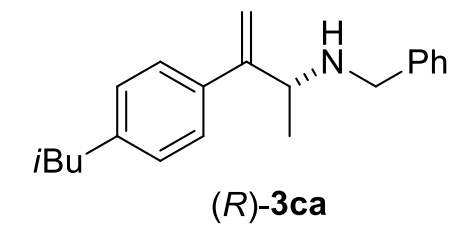

${ }^{13} \mathrm{C}\left\{{ }^{1} \mathrm{H}\right\}$ NMR $\left(100 \mathrm{MHz}, \mathrm{CDCl}_{3}\right)$
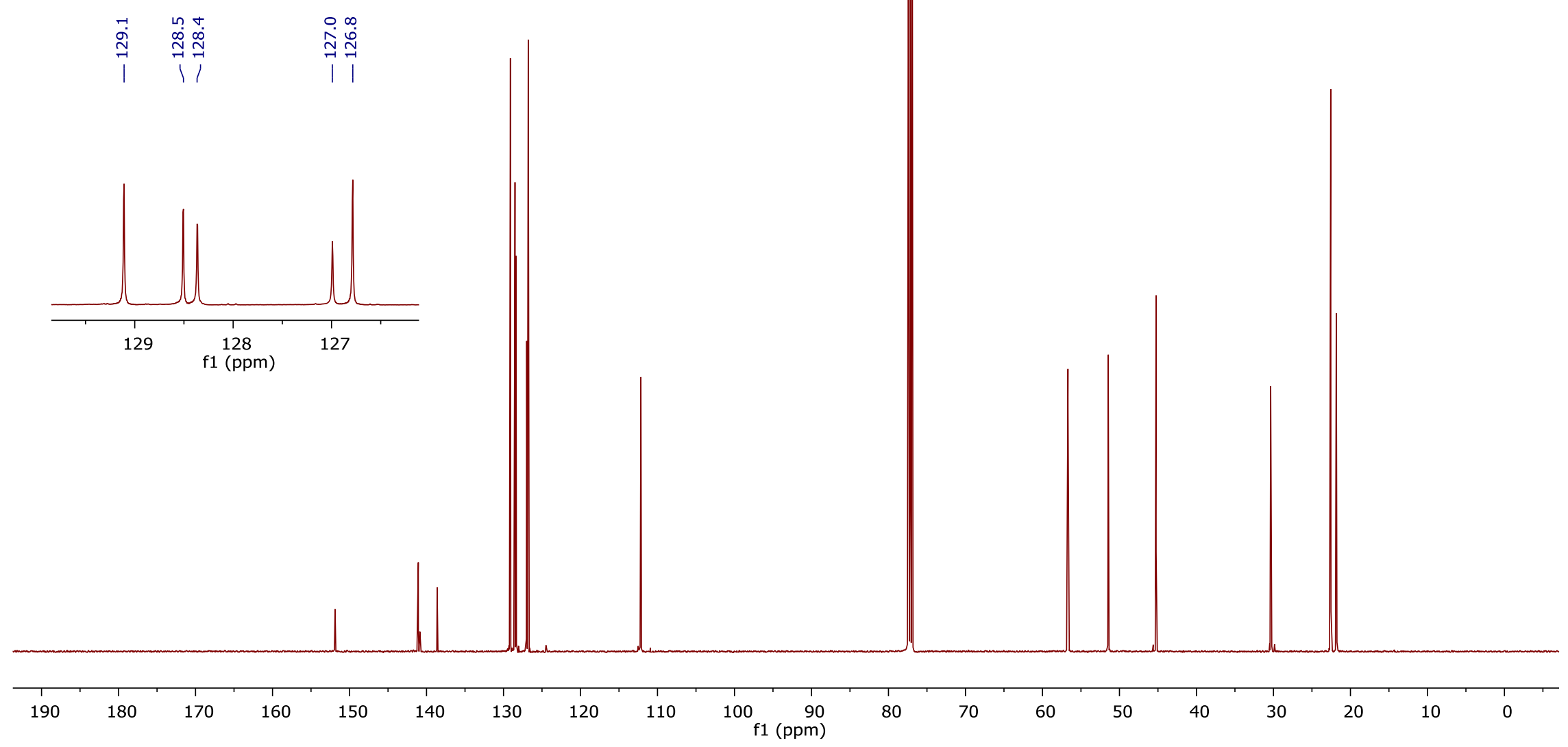


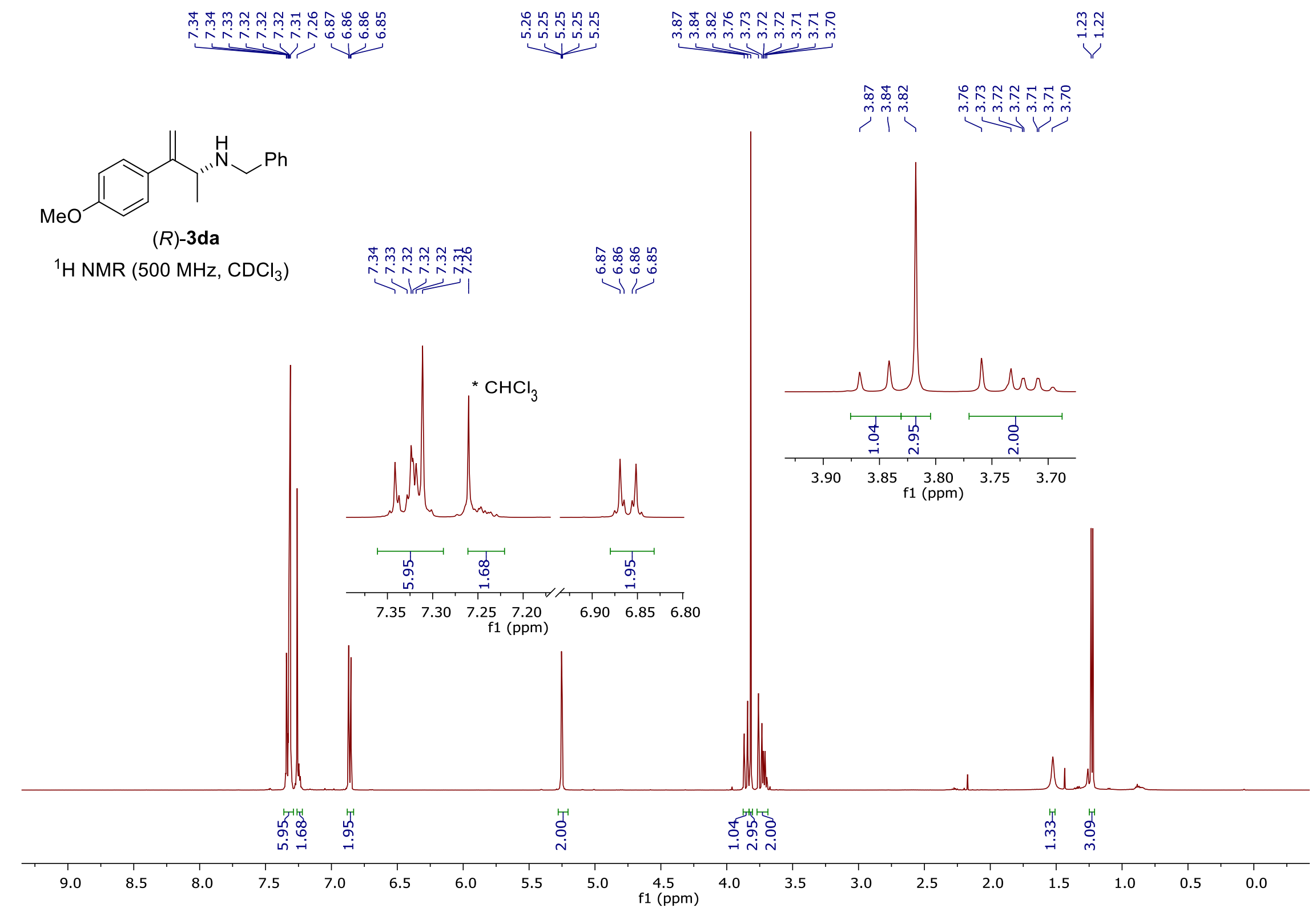

S101 


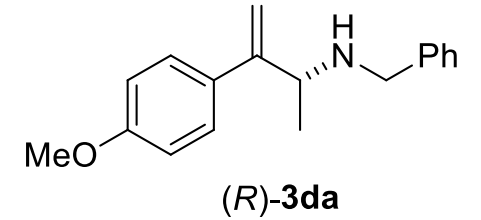

${ }^{13} \mathrm{C}\left\{{ }^{1} \mathrm{H}\right\}$ NMR $\left(130 \mathrm{MHz}, \mathrm{CDCl}_{3}\right)$

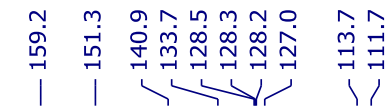

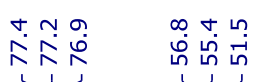

ए

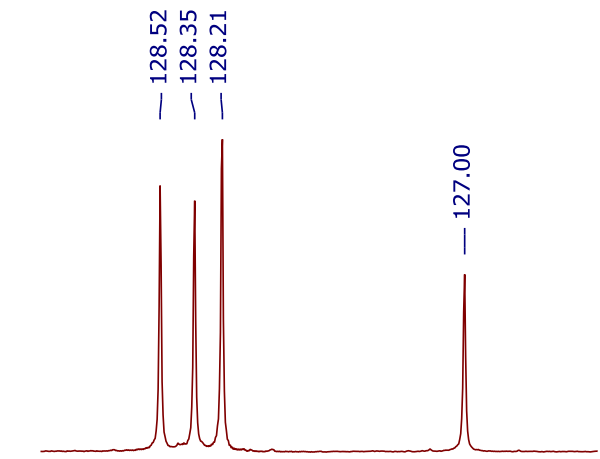

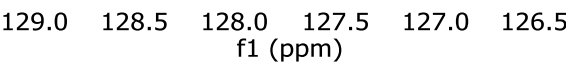

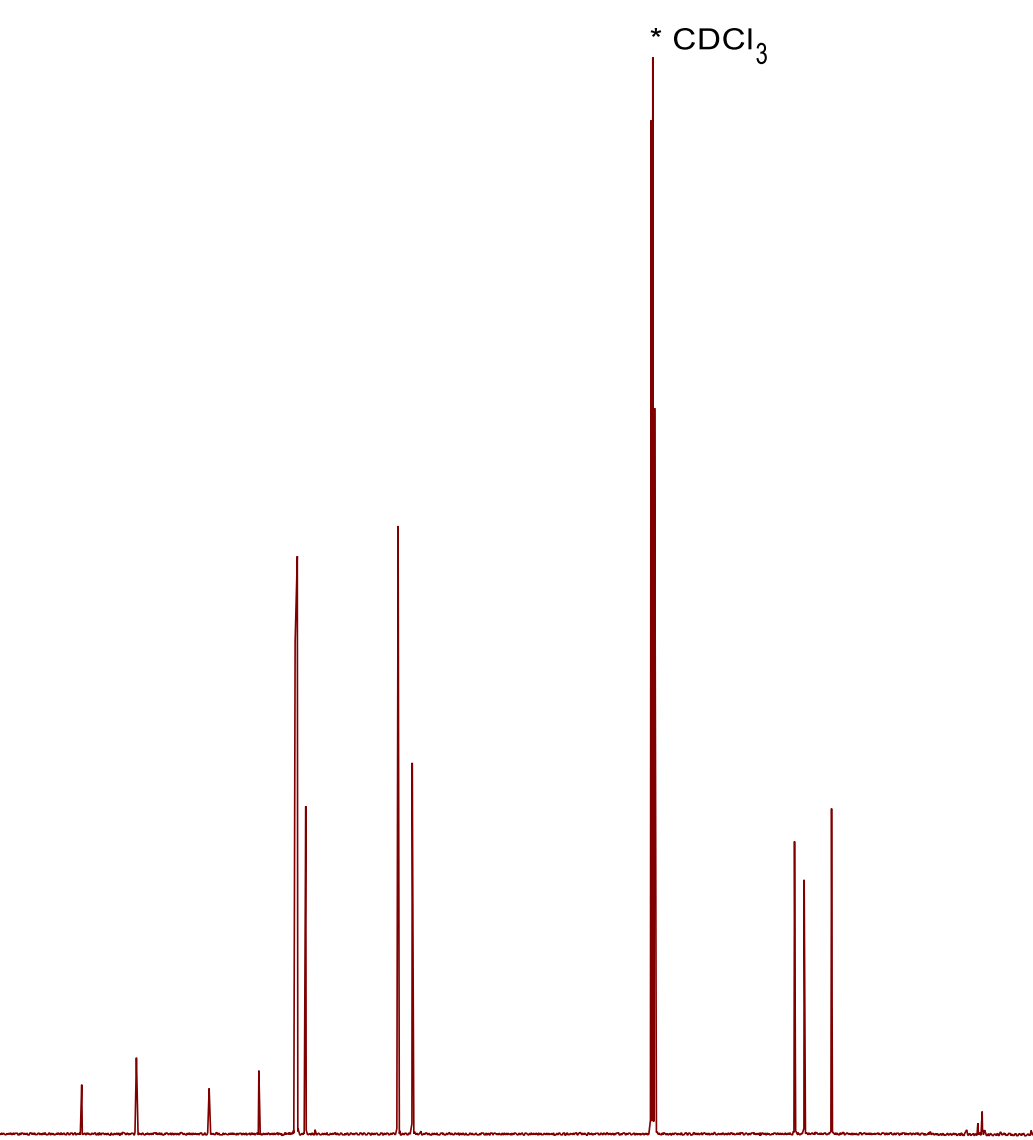

$\begin{array}{llllllllllllllllll}260 & 250 & 240 & 230 & 220 & 210 & 200 & 190 & 180 & 170 & 160 & 150 & 140 & 130 & 120 & 110 & 100 & 90\end{array}$

f1 (ppm) 


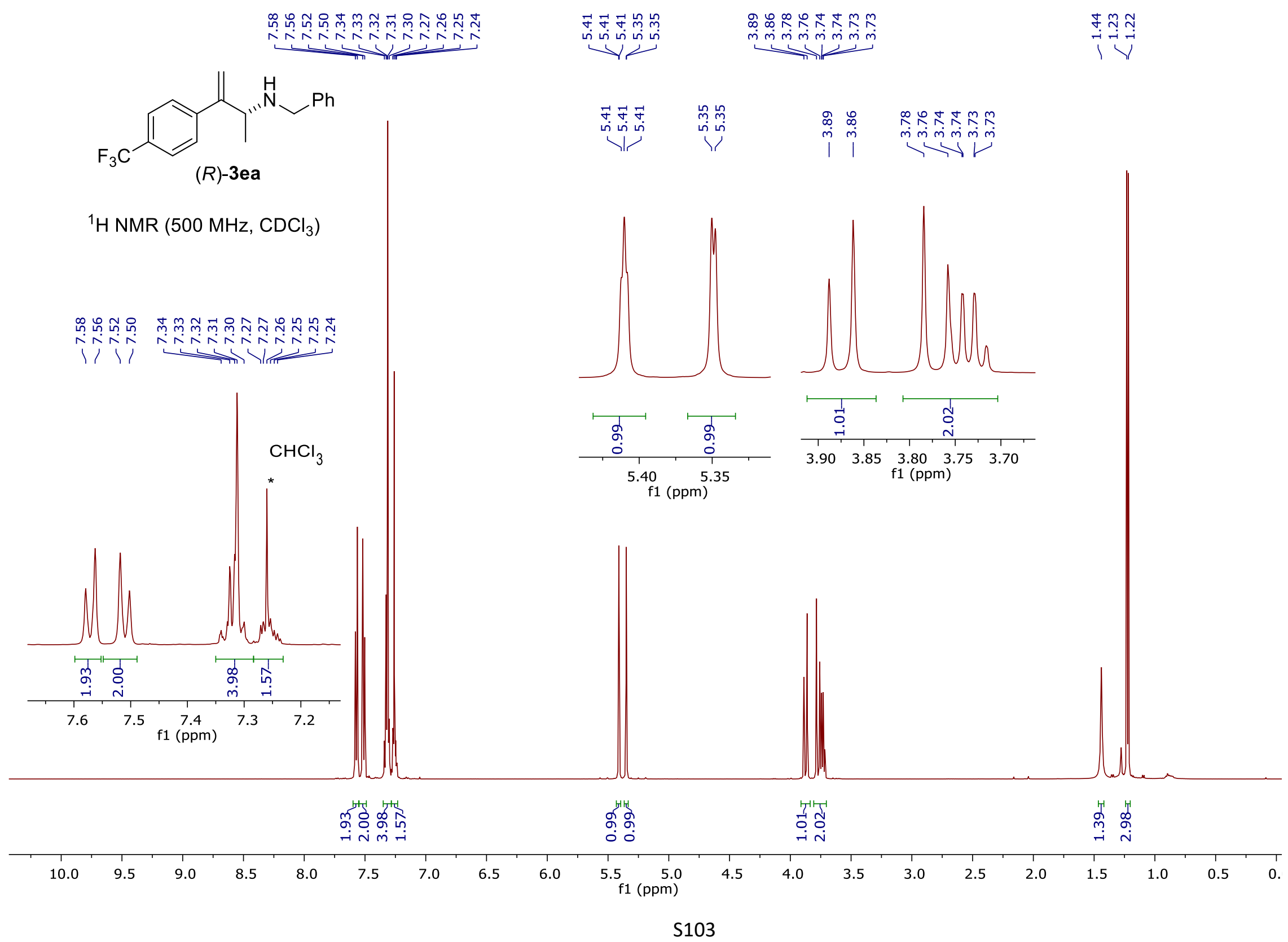




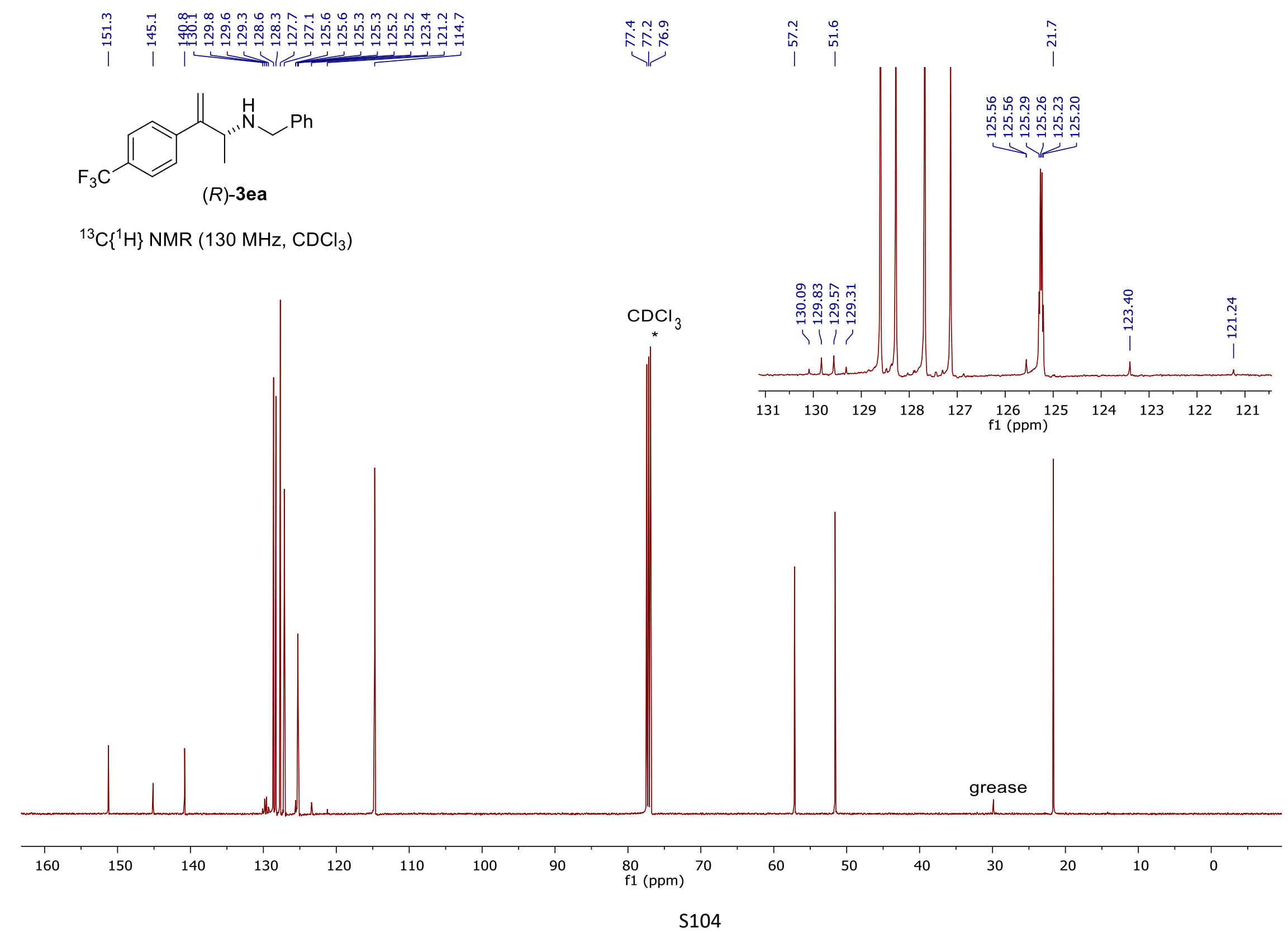




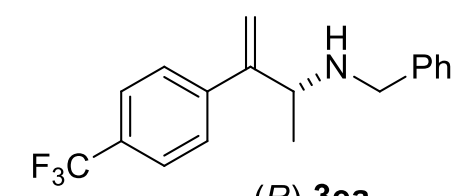

$(R)-3 \mathbf{e a}$

${ }^{19} \mathrm{~F}\left\{{ }^{1} \mathrm{H}\right\}$ NMR $\left(280 \mathrm{MHz}, \mathrm{CDCl}_{3}\right)$

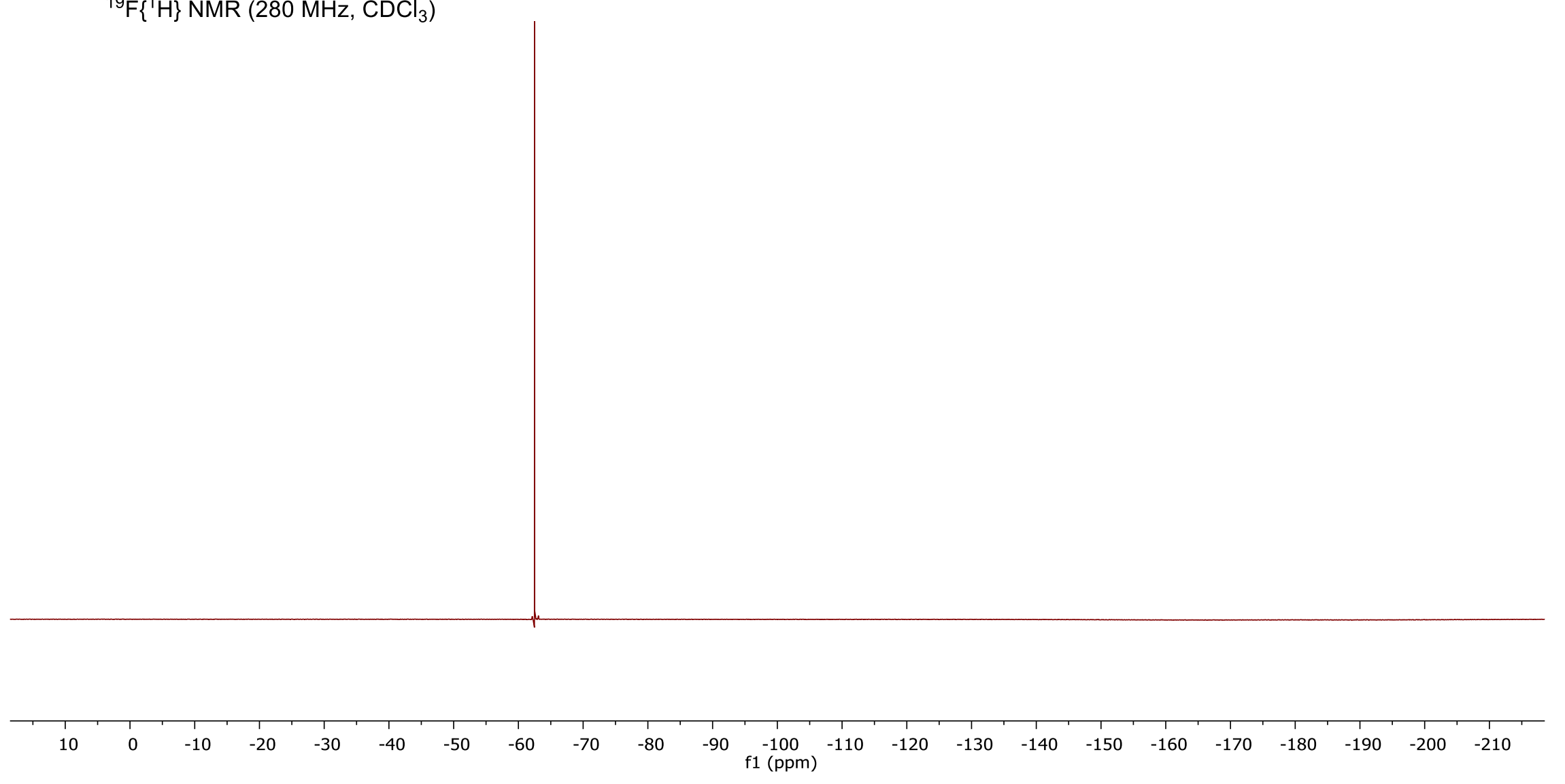




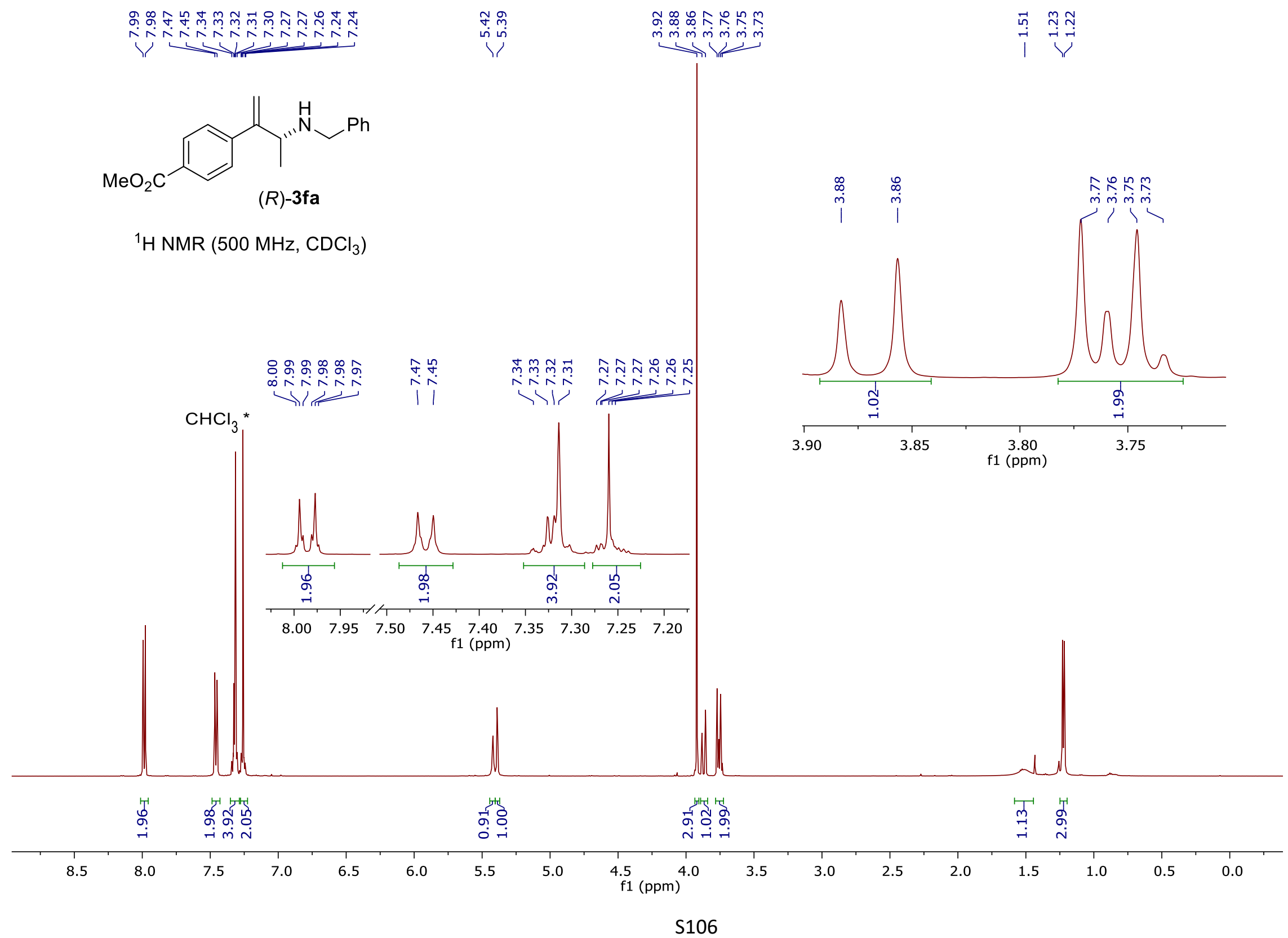




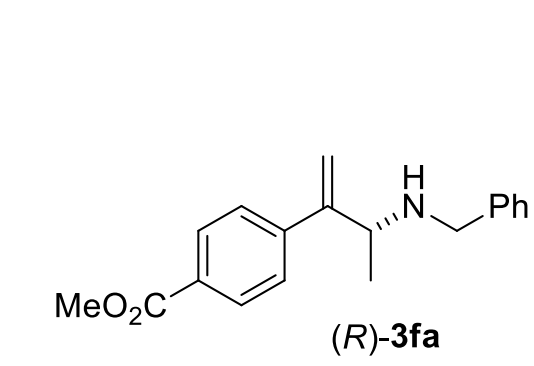

${ }^{13} \mathrm{C}\left\{{ }^{1} \mathrm{H}\right\}$ NMR $\left(130 \mathrm{MHz}, \mathrm{CDCl}_{3}\right)$

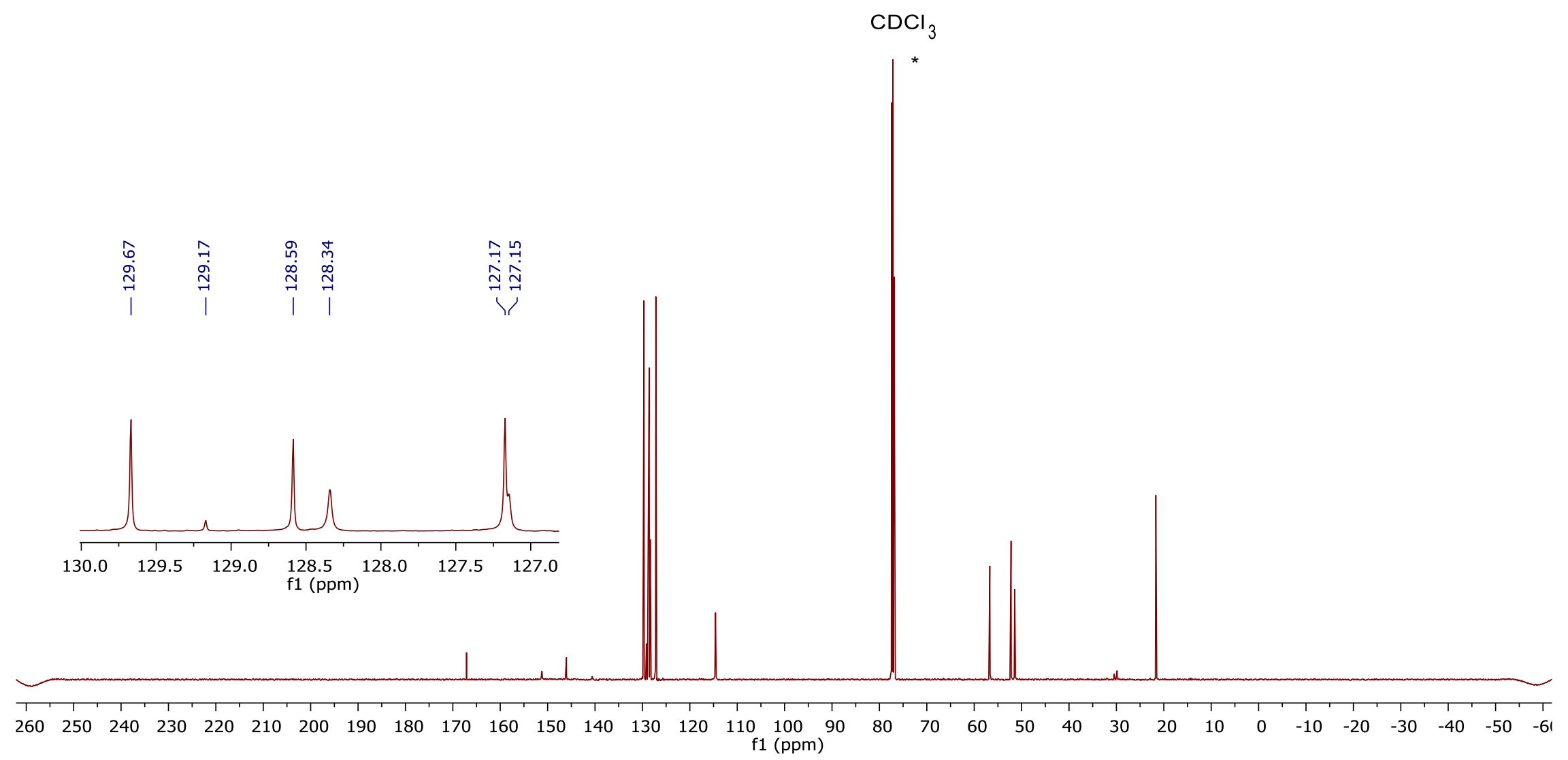




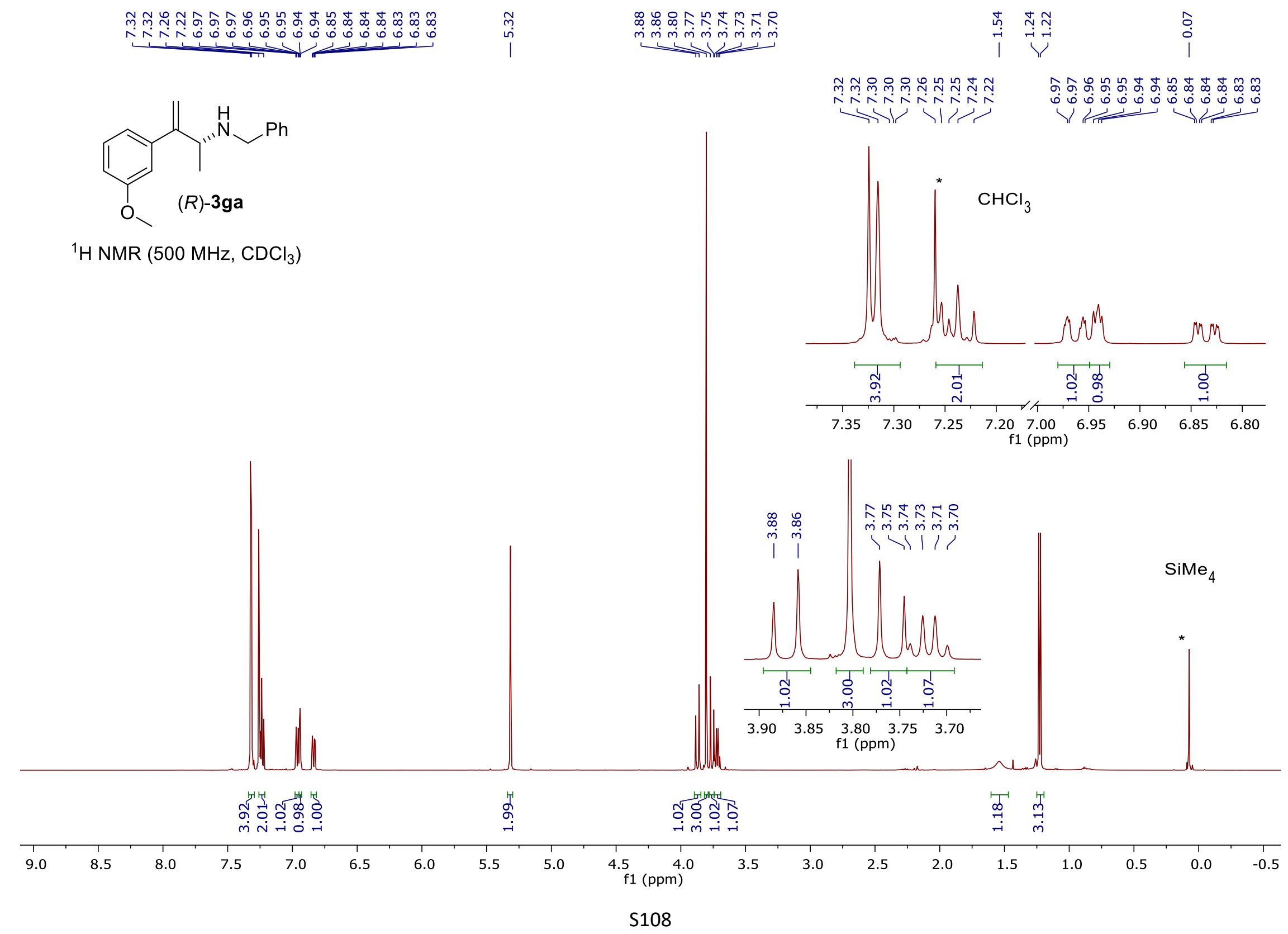




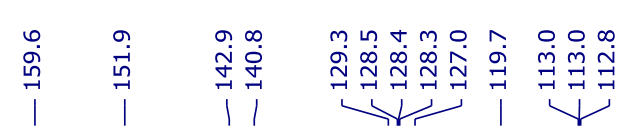

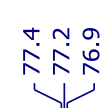

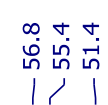

$\stackrel{i}{i}$

$\stackrel{\sim}{\sim}$

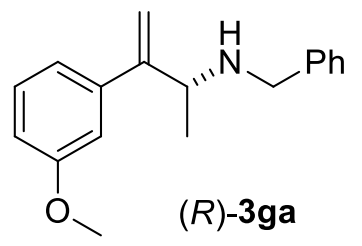

${ }^{13} \mathrm{C}\left\{{ }^{1} \mathrm{H}\right\} \mathrm{NMR}\left(130 \mathrm{MHz}, \mathrm{CDCl}_{3}\right)$

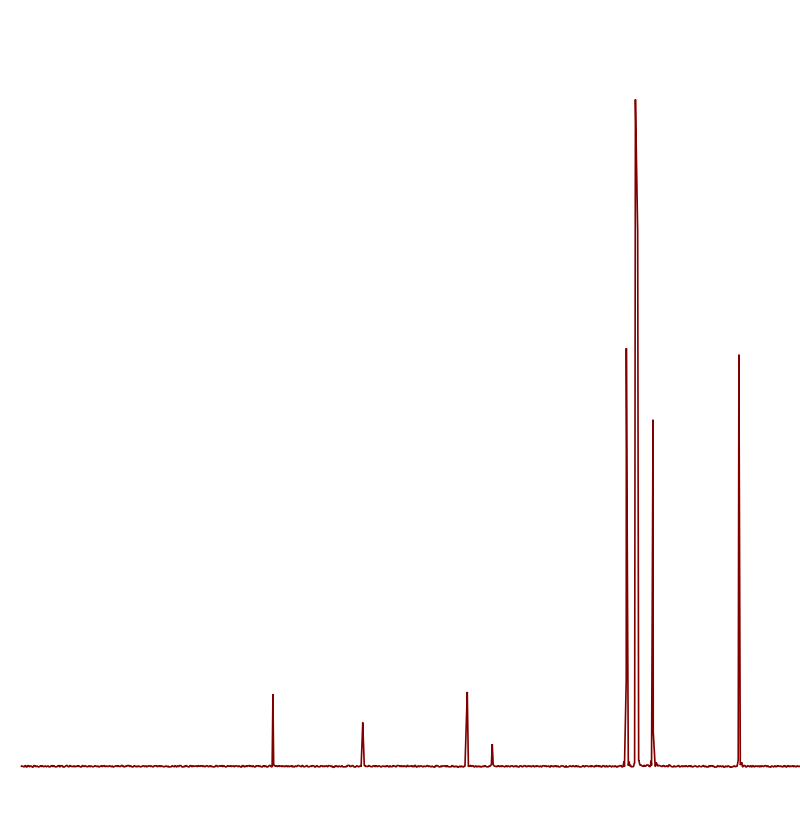

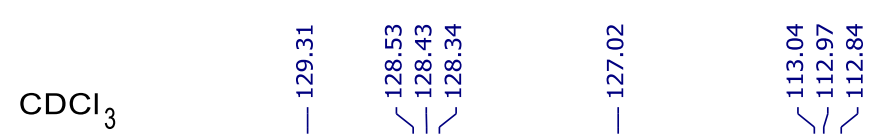
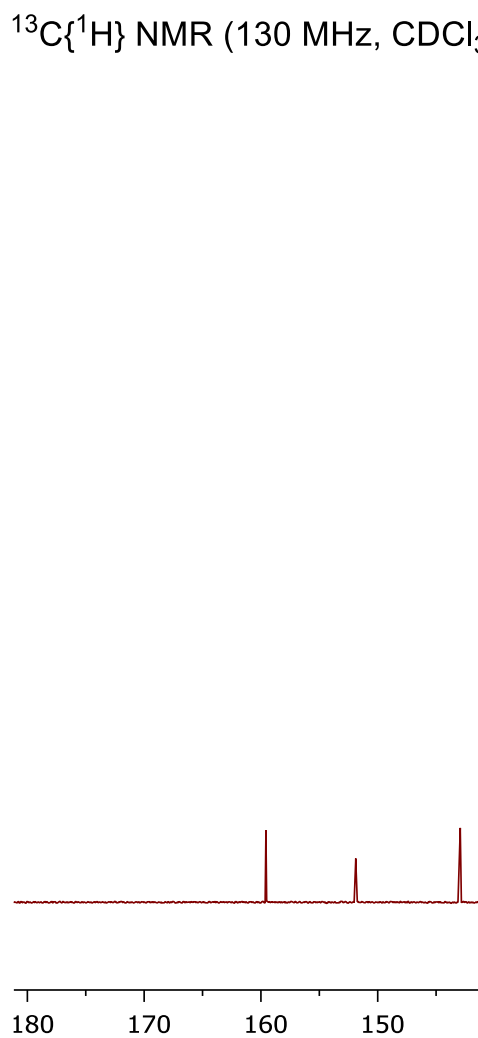

$150 \quad 140$

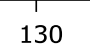

120

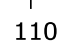

100

$90 \quad 80$

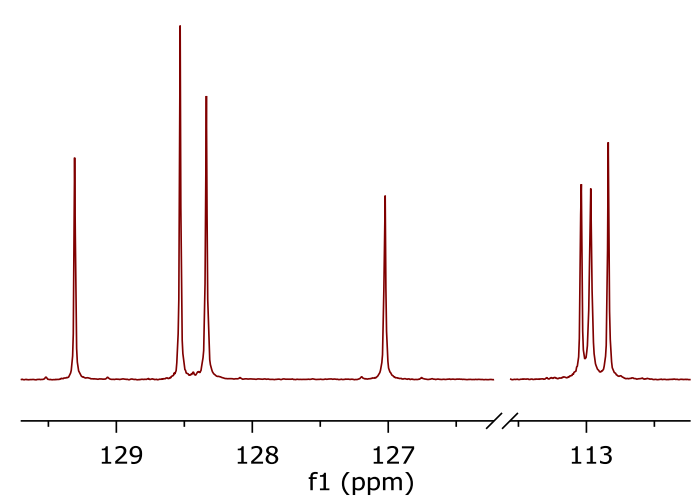

S109 


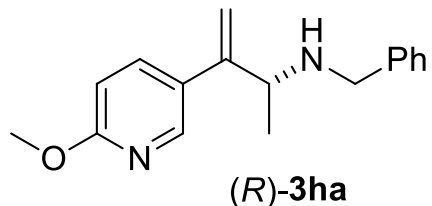

${ }^{1} \mathrm{H}$ NMR $\left(500 \mathrm{MHz}, \mathrm{CDCl}_{3}\right)$

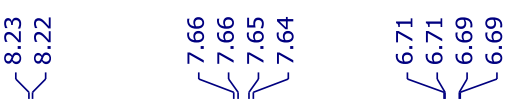

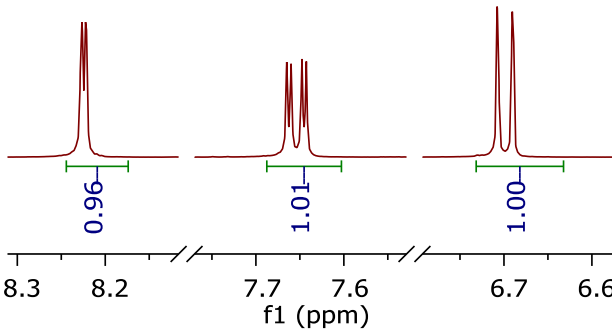

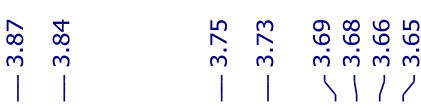

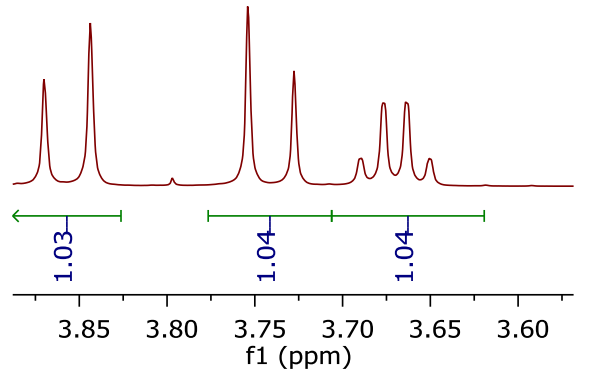

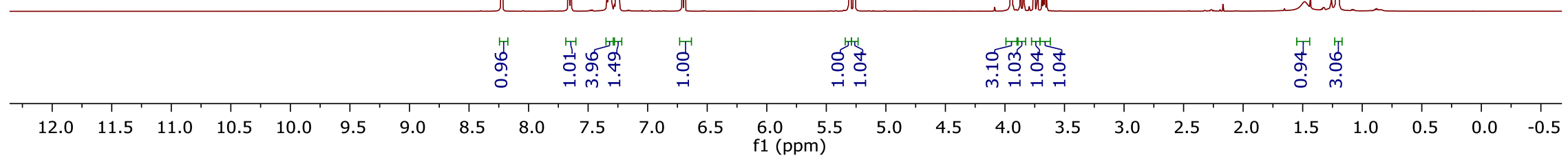




每)-3ha

${ }^{13} \mathrm{C}\left\{{ }^{1} \mathrm{H}\right\}$ NMR (130 MHz, $\mathrm{CDCl}_{3}$ )

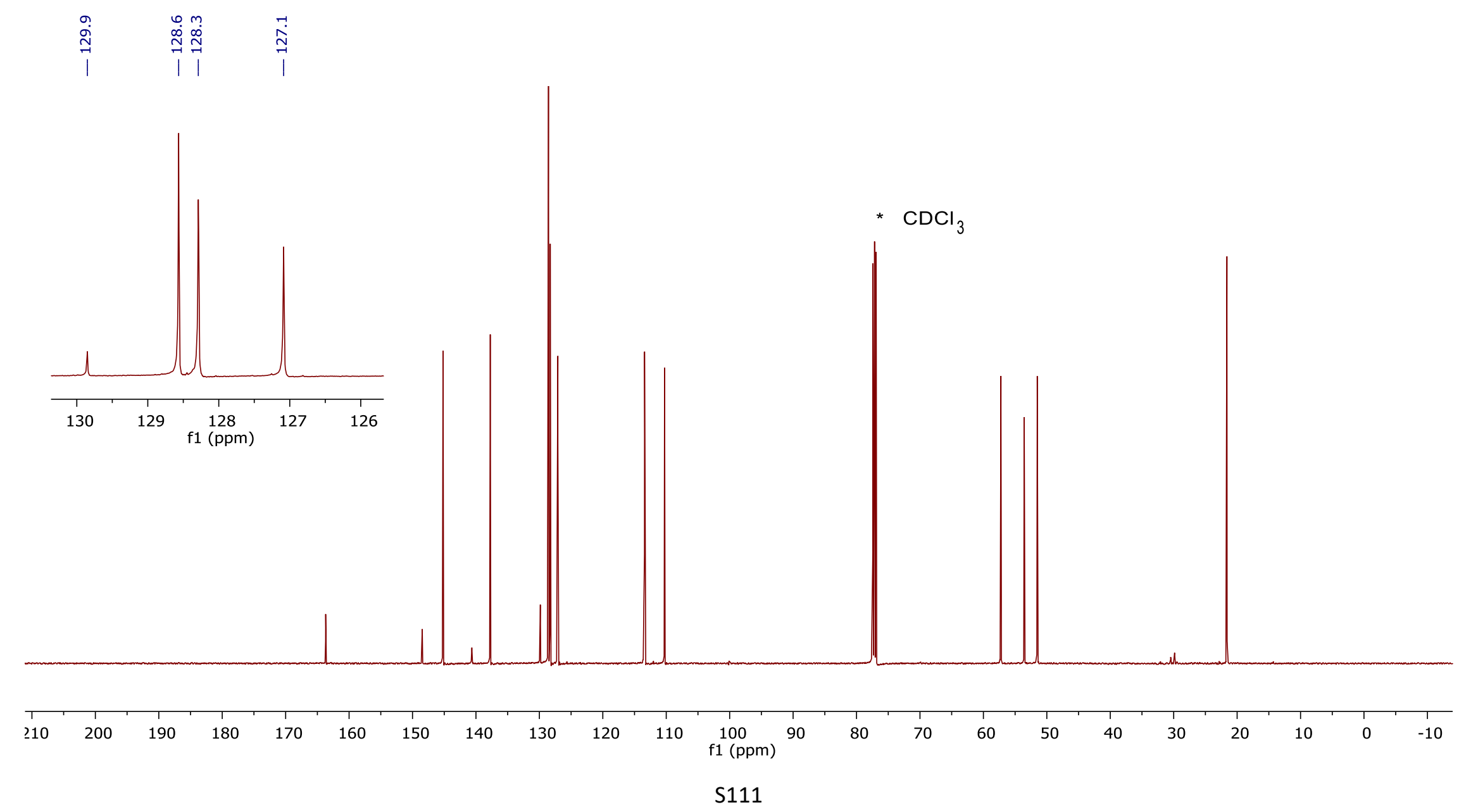




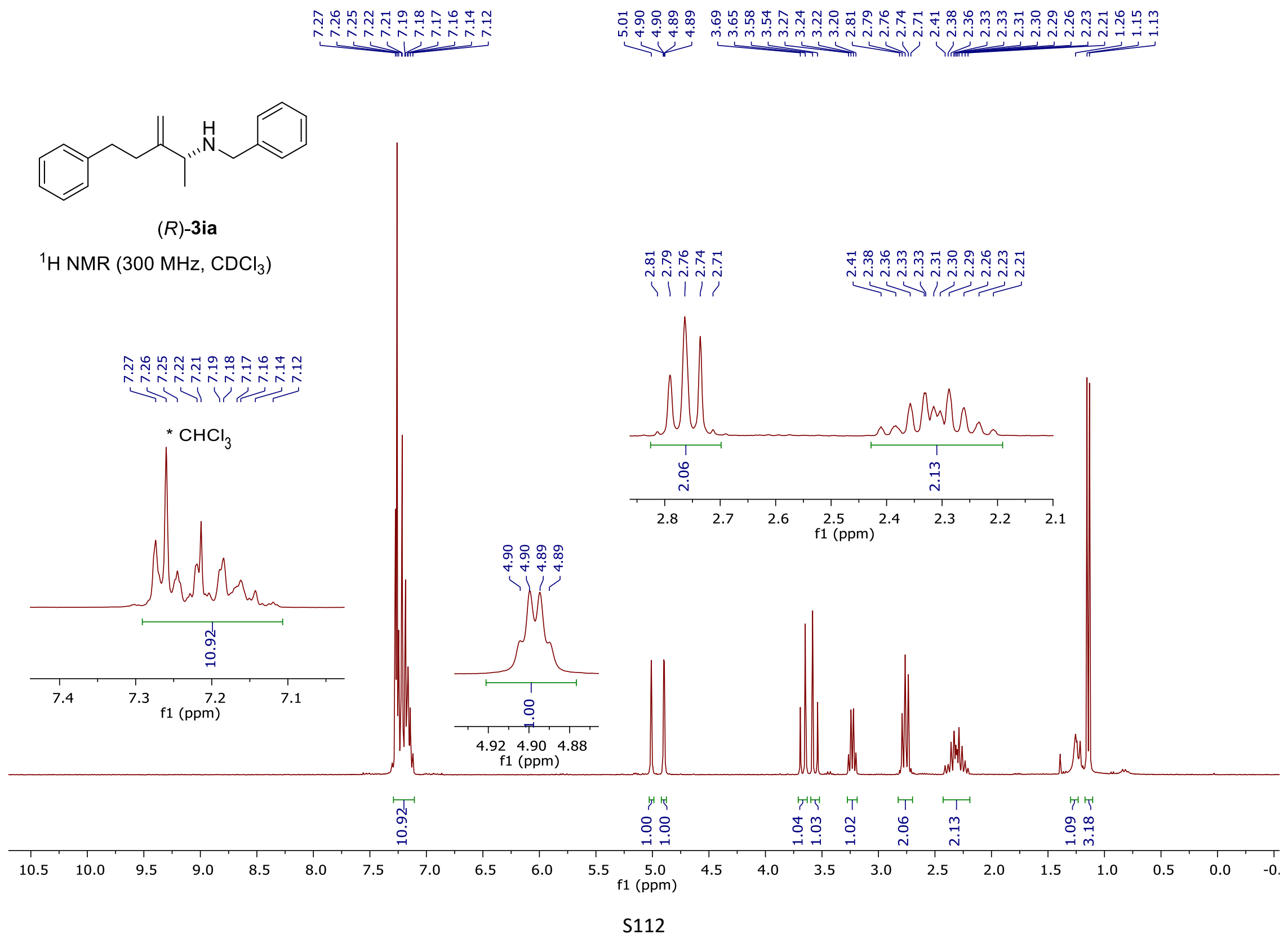



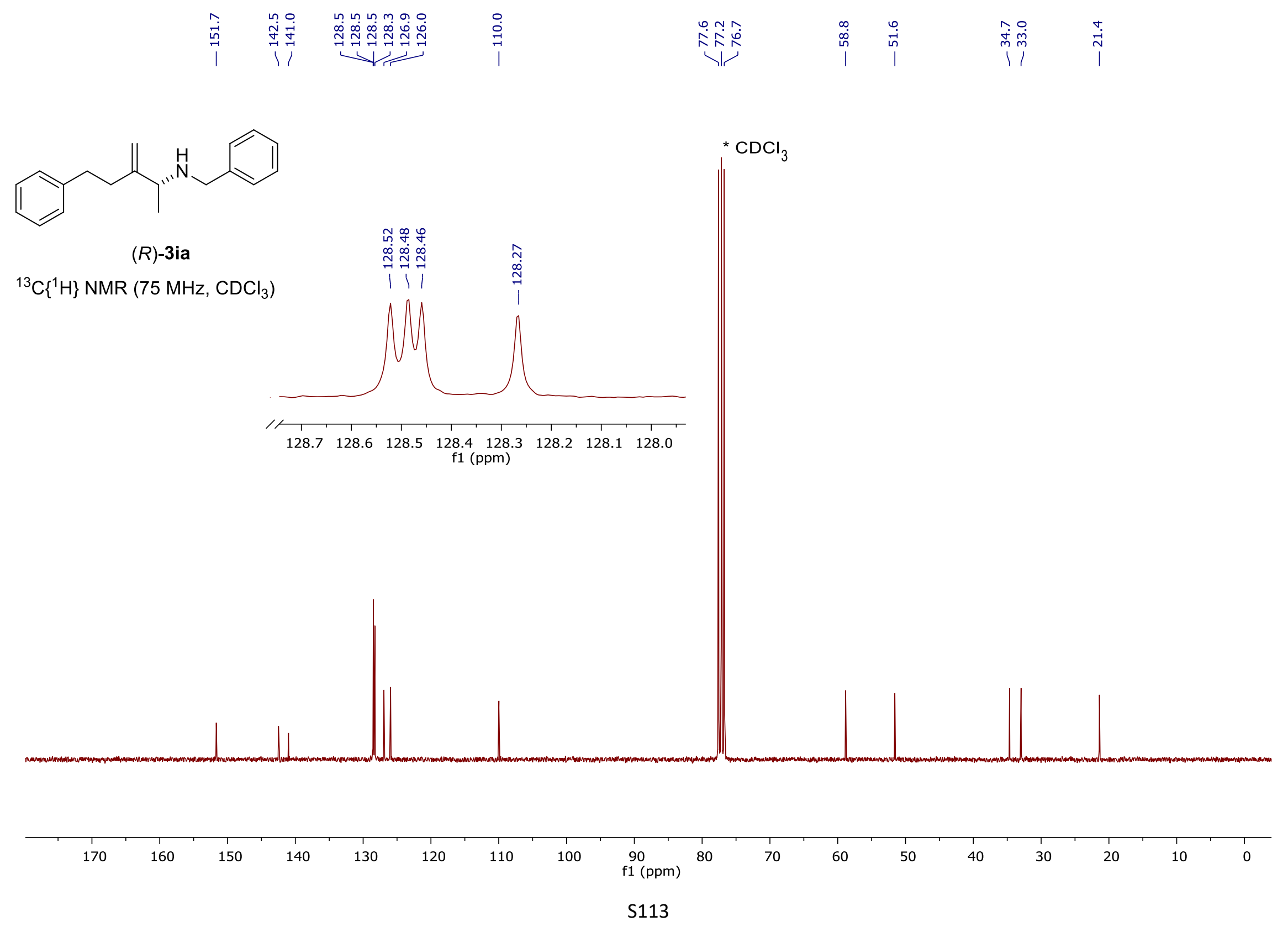


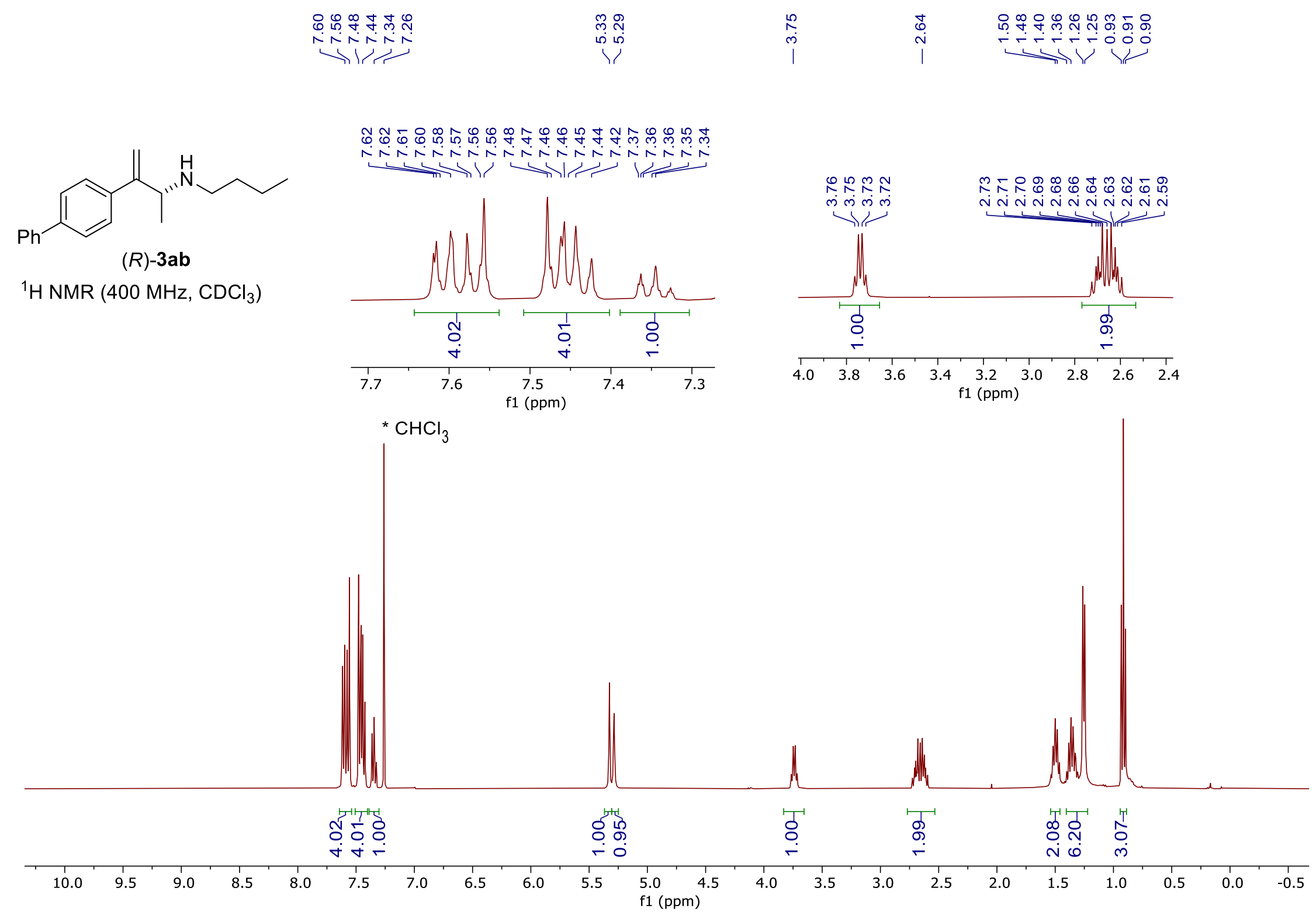




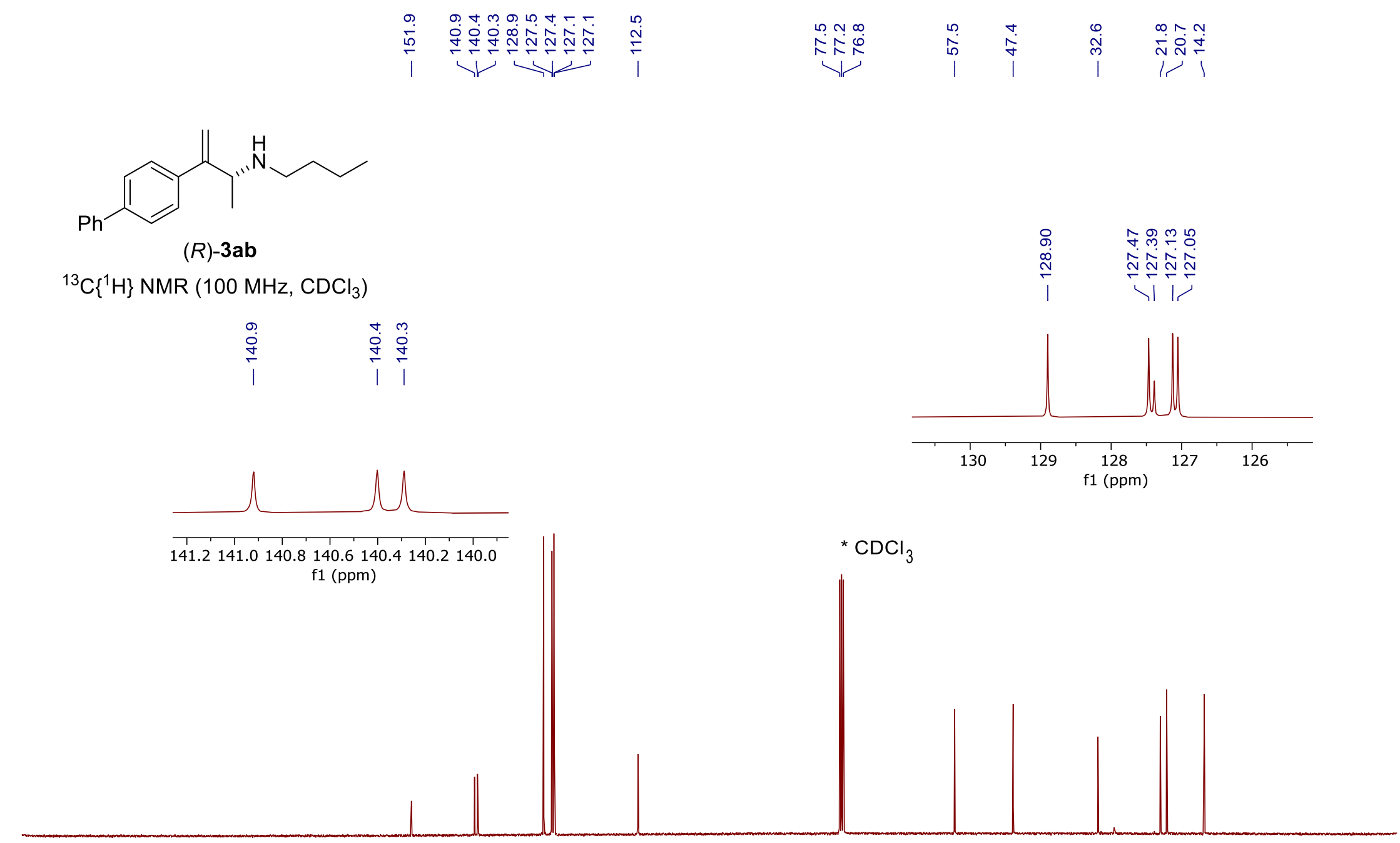

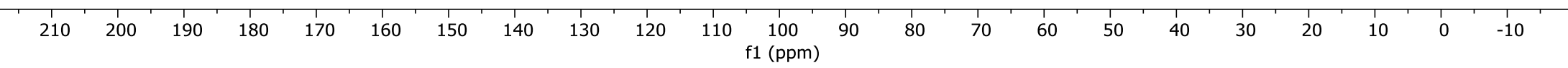




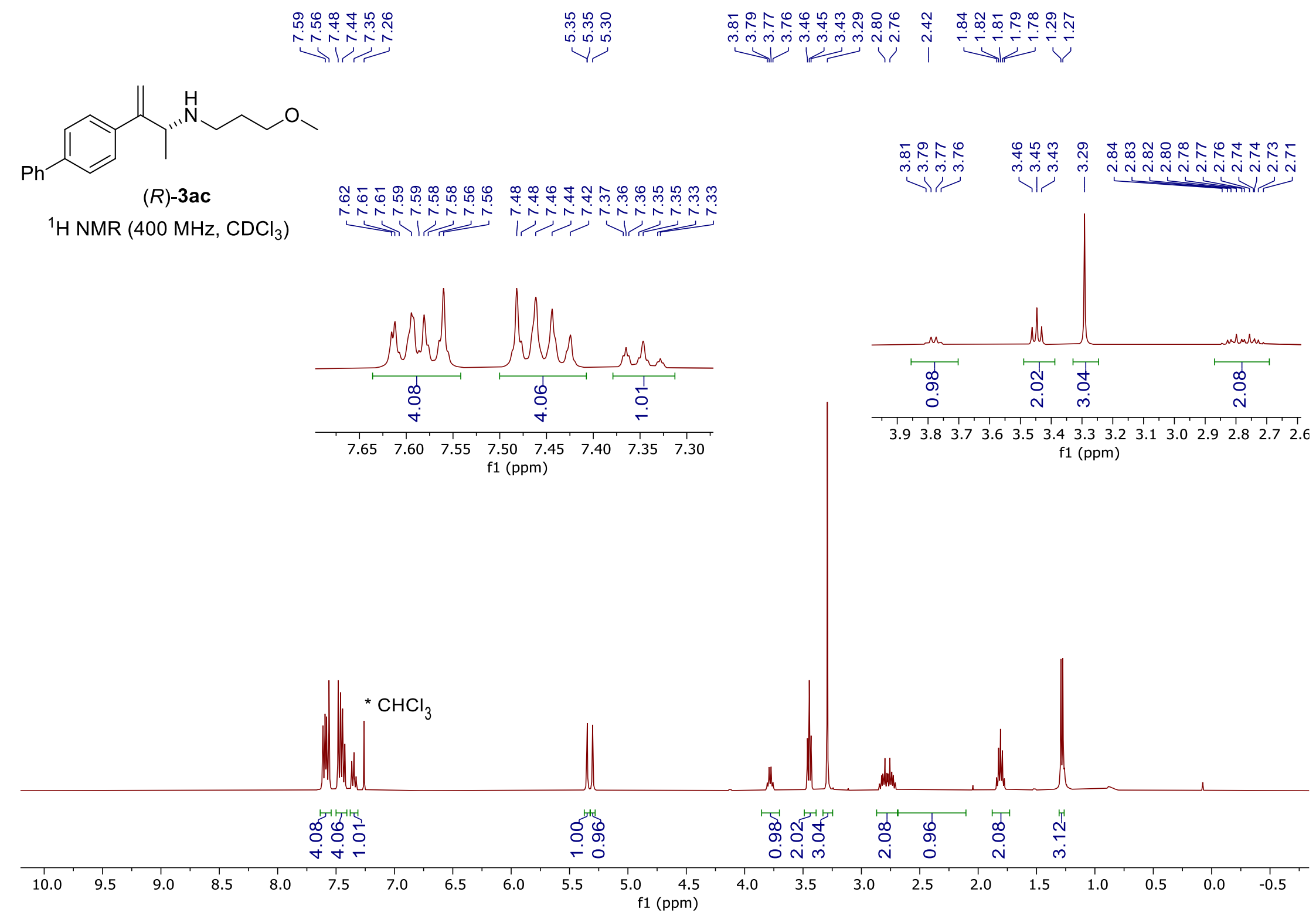




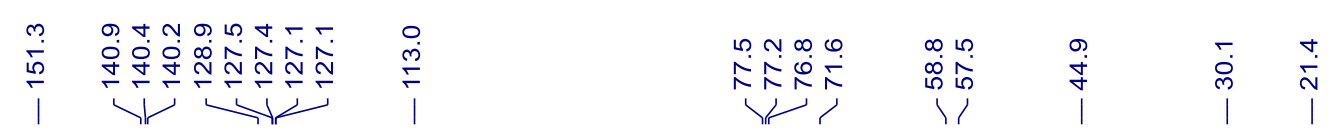

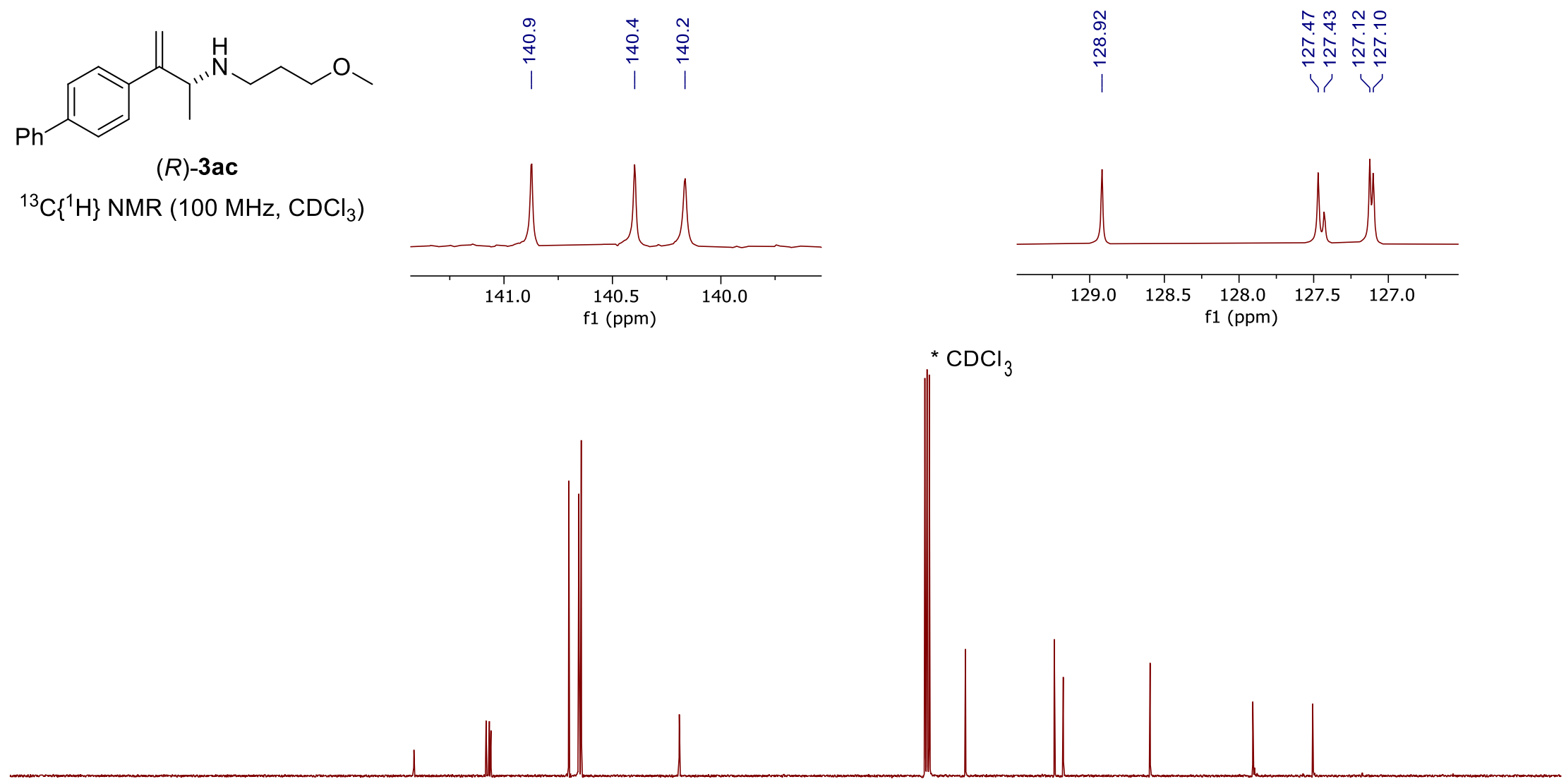

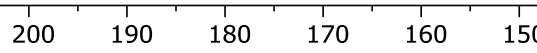

130

$120 \quad 110$

$\begin{array}{lll}100 & 90 & 80\end{array}$

70

$60 \quad 50$

$40 \quad 30$

$\begin{array}{lllll}1 & 10 & 0 & -10\end{array}$ 


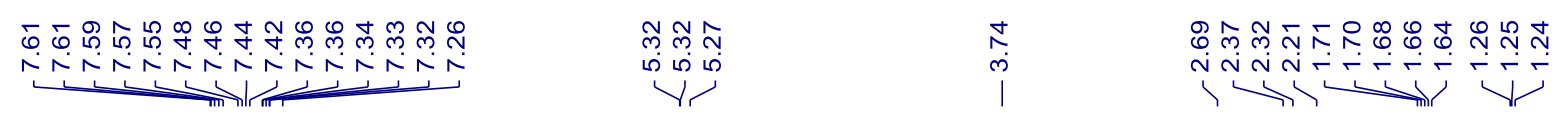<smiles>C=C(c1ccc(-c2ccccc2)cc1)[C@H](C)NCCCN(C)C</smiles>

(R)-3ad

${ }^{1} \mathrm{H}$ NMR $\left(400 \mathrm{MHz}, \mathrm{CDCl}_{3}\right)$

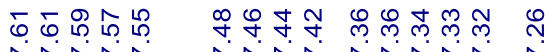

र份却 ।
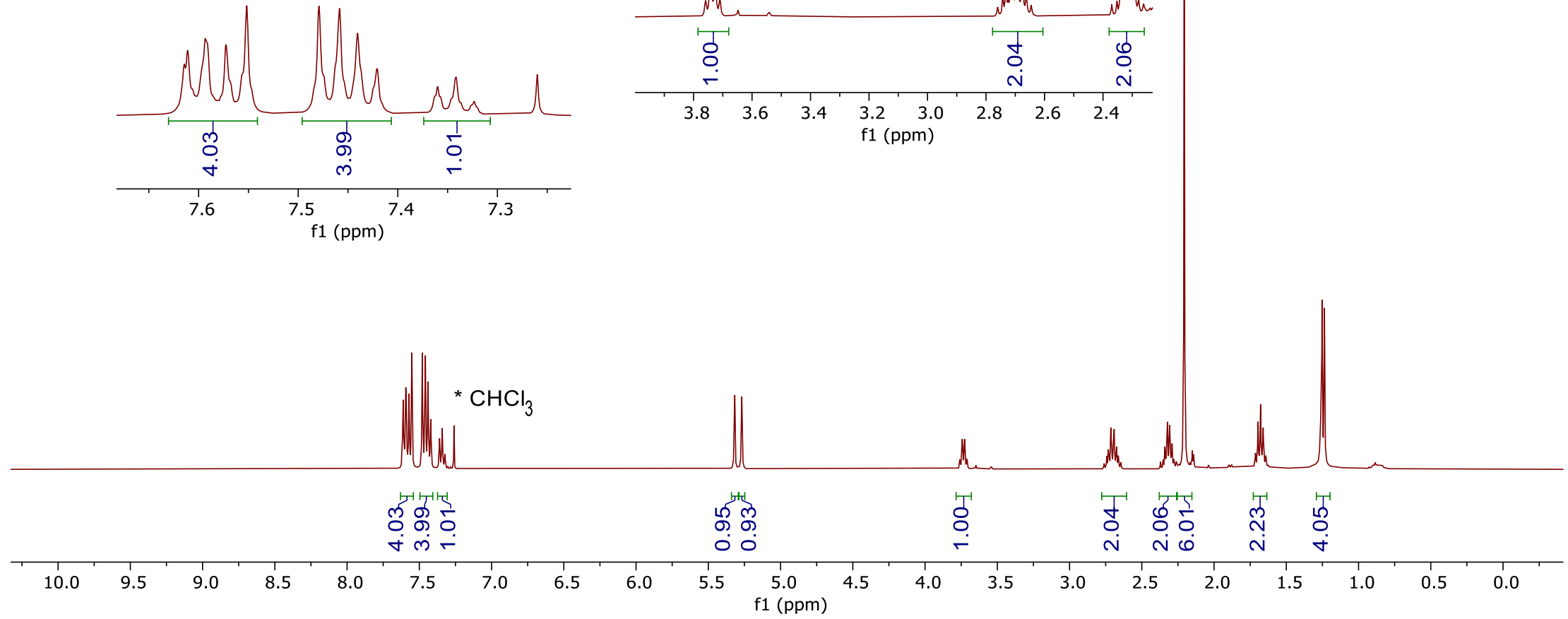


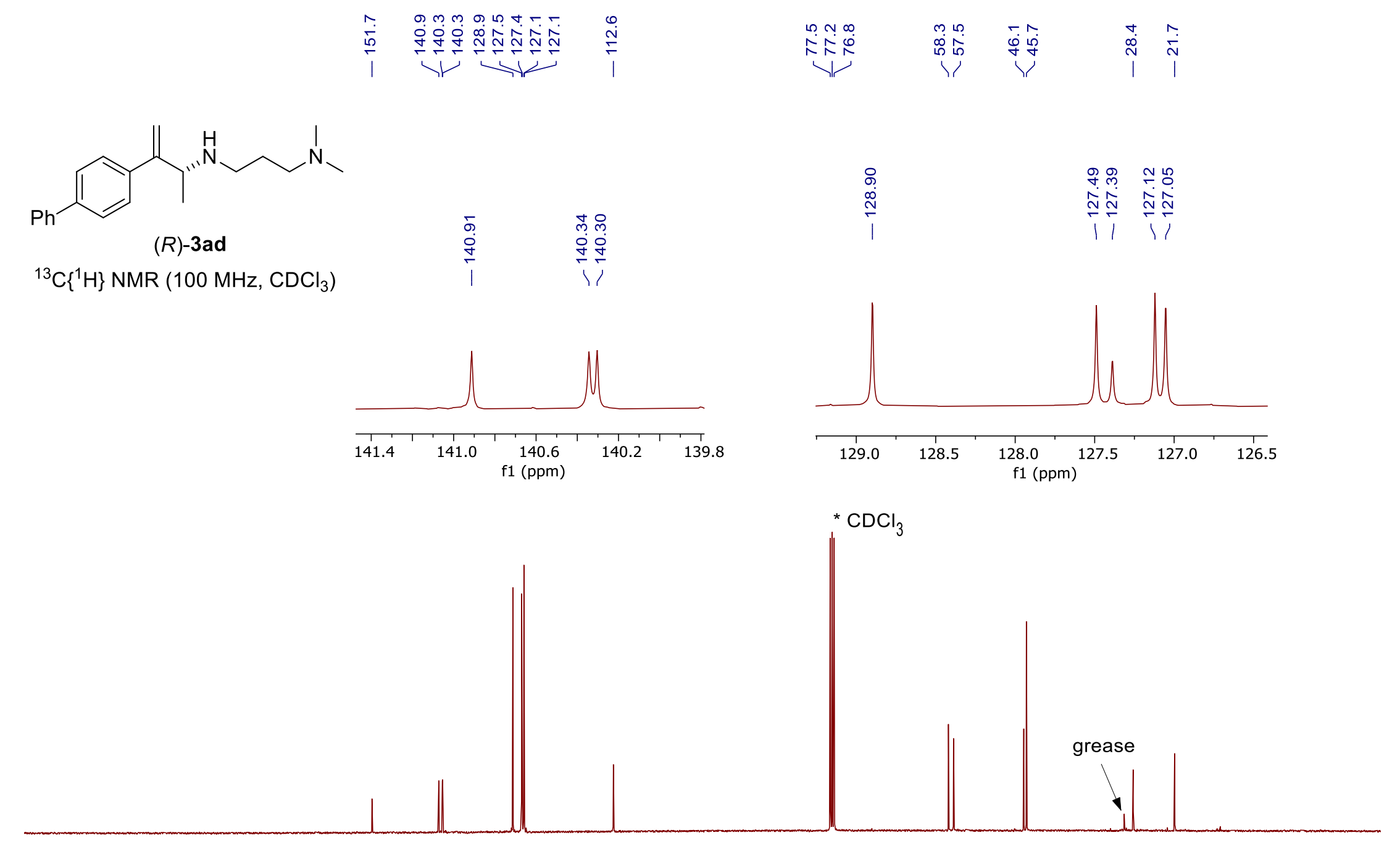

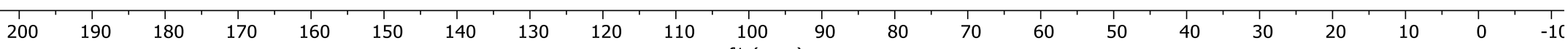




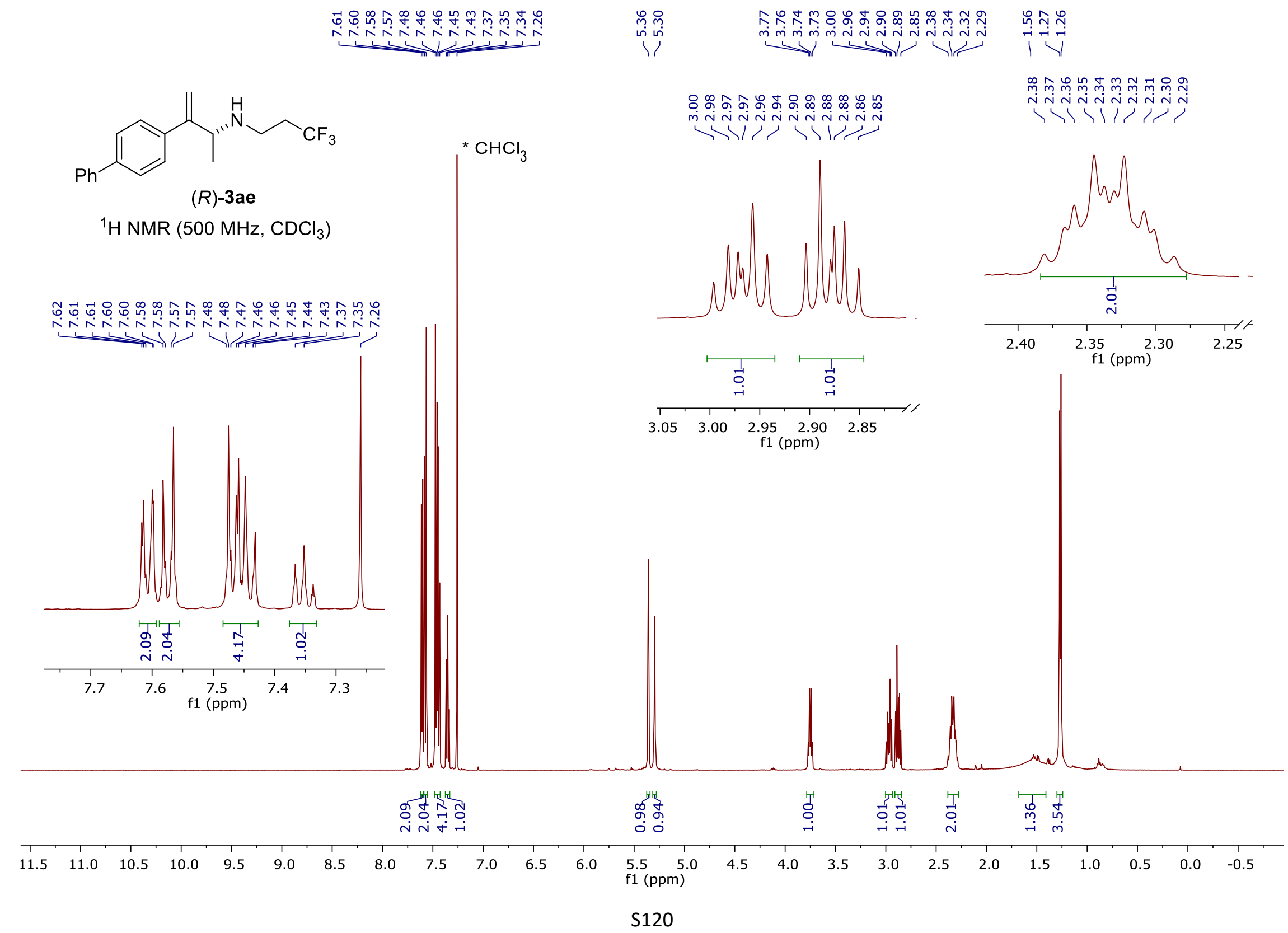




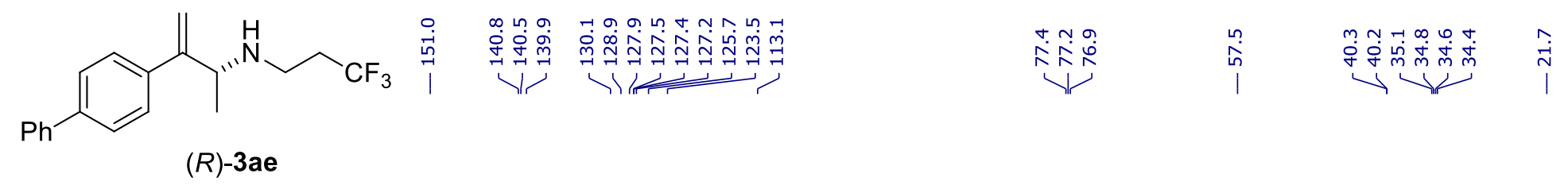

${ }^{13} \mathrm{C}\left\{{ }^{1} \mathrm{H}\right\} \mathrm{NMR}\left(130 \mathrm{MHz}, \mathrm{CDCl}_{3}\right)$

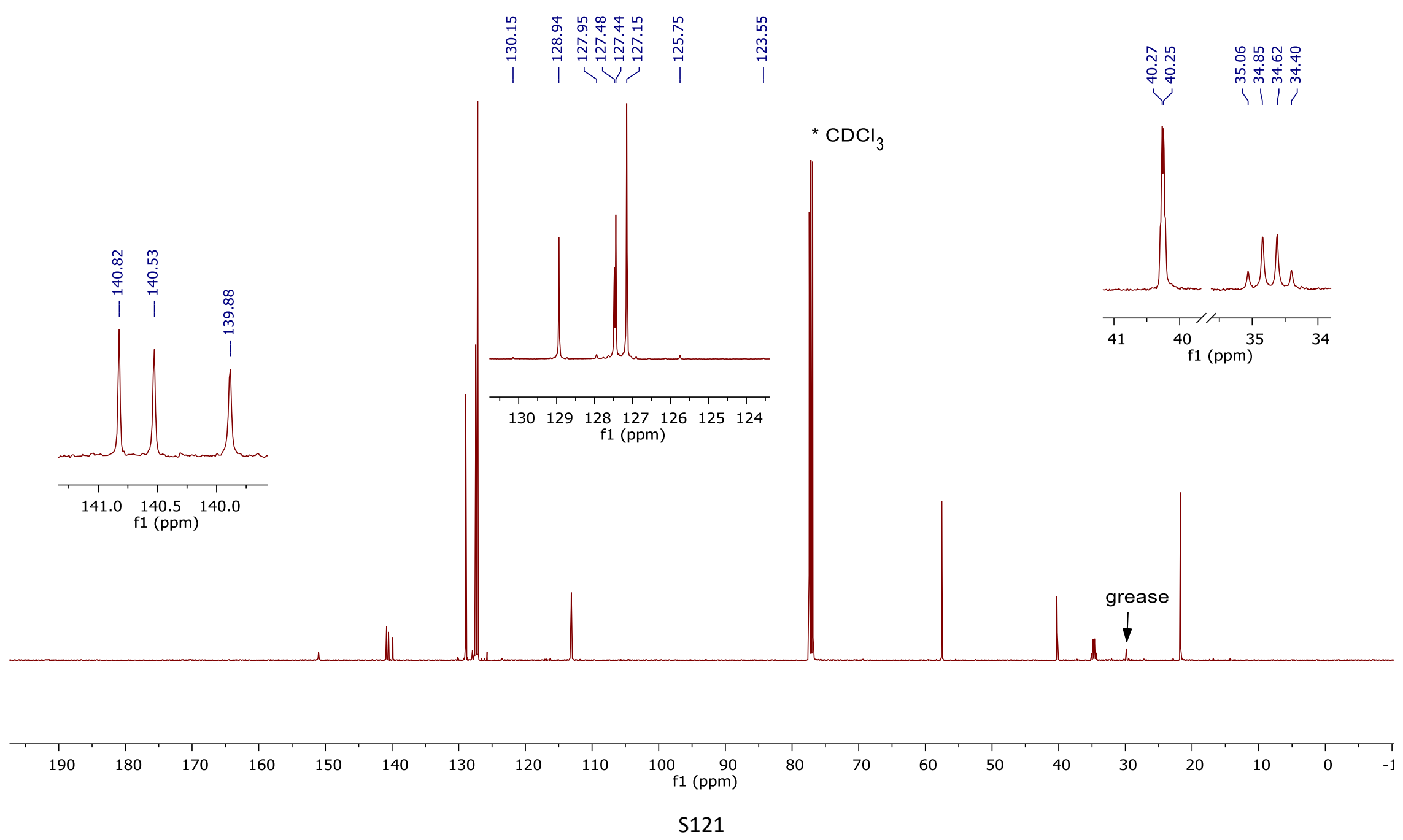




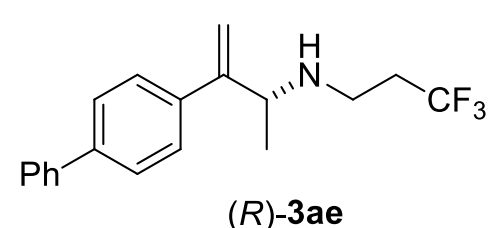

${ }^{19} \mathrm{~F}\left\{{ }^{1} \mathrm{H}\right\} \mathrm{NMR}\left(280 \mathrm{MHz}, \mathrm{CDCl}_{3}\right)$

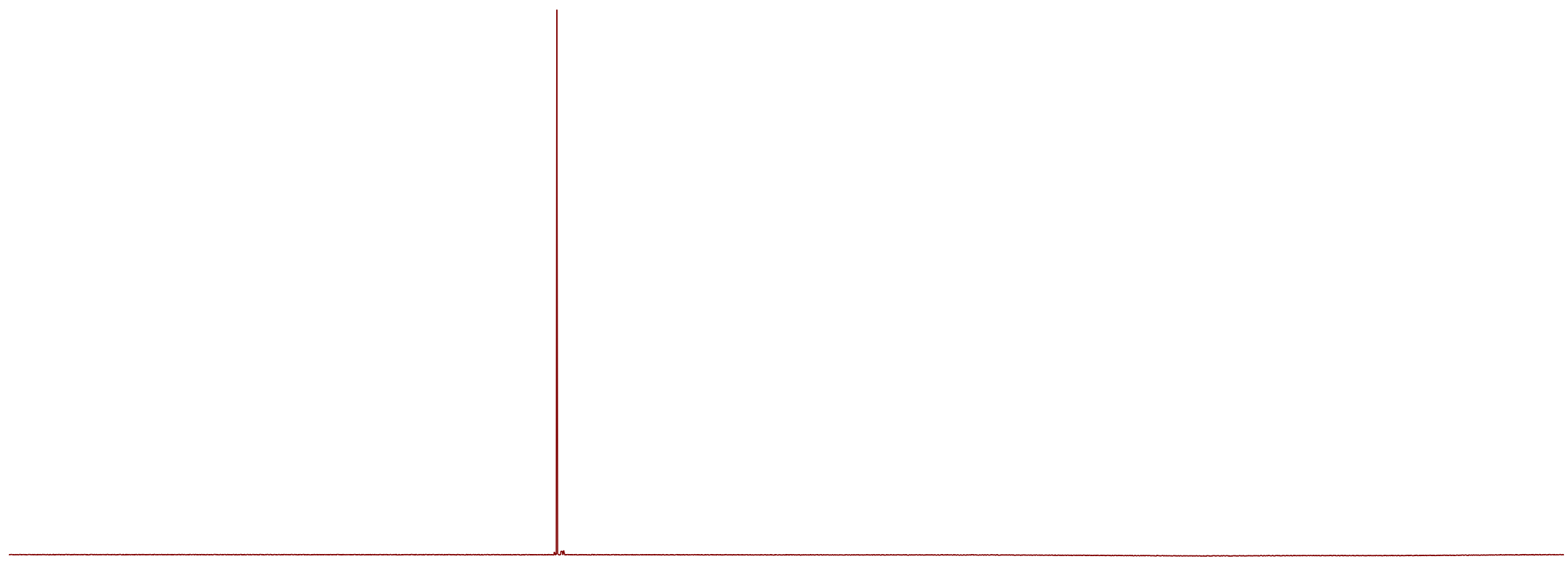

$\begin{array}{lllllllllllllllllllllllllllll}10 & 0 & -10 & -20 & -30 & -40 & -50 & -60 & -70 & -80 & -90 & -100 & -110 & -120 & -130 & -140 & -150 & -160 & -170 & -180 & -190 & -200 & -210 & \end{array}$




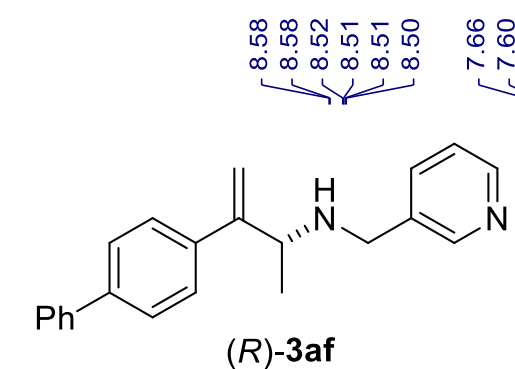

${ }^{1} \mathrm{H} \mathrm{NMR}\left(400 \mathrm{MHz}, \mathrm{CDCl}_{3}\right)$
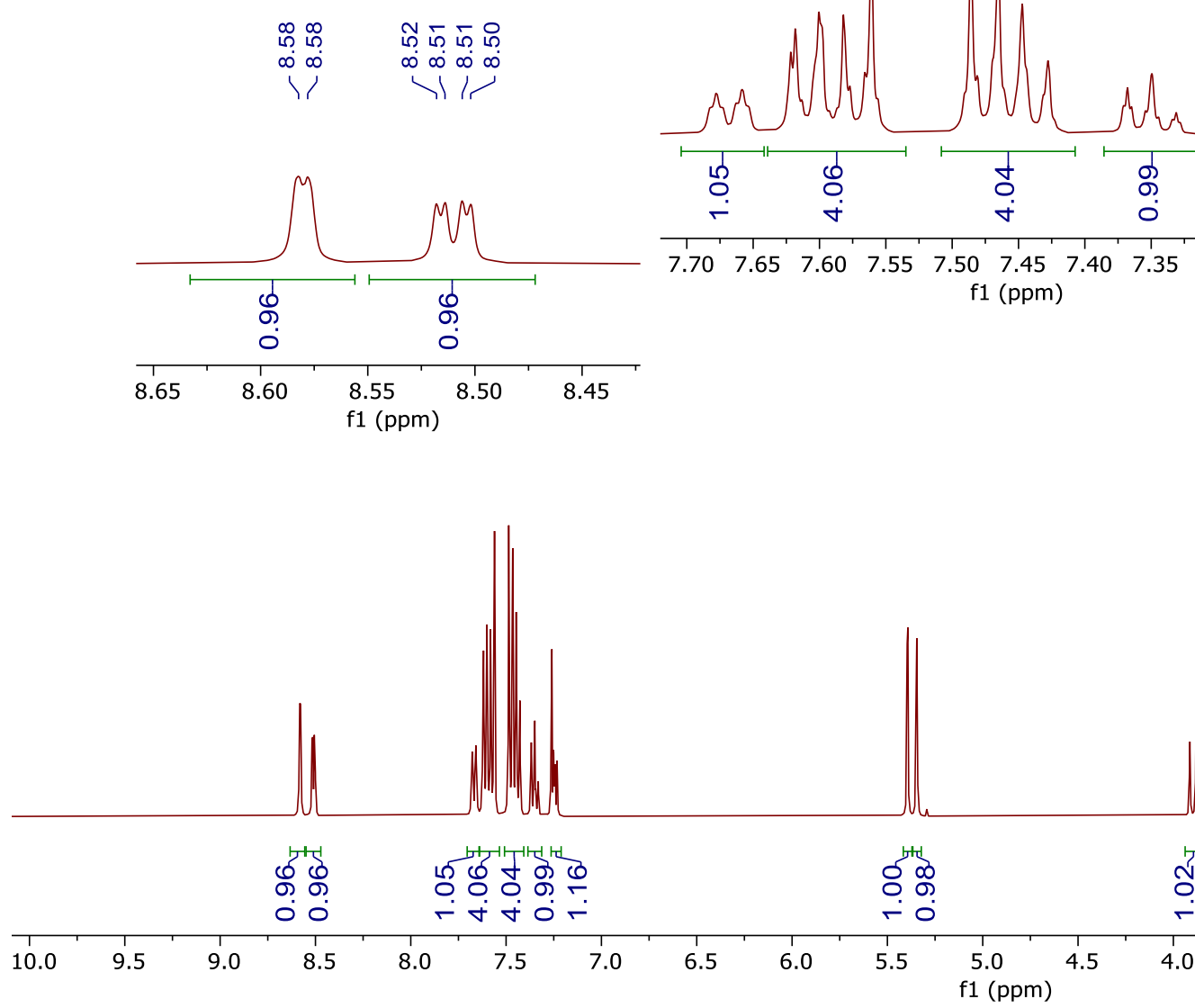

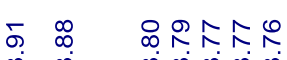

i लंखmं
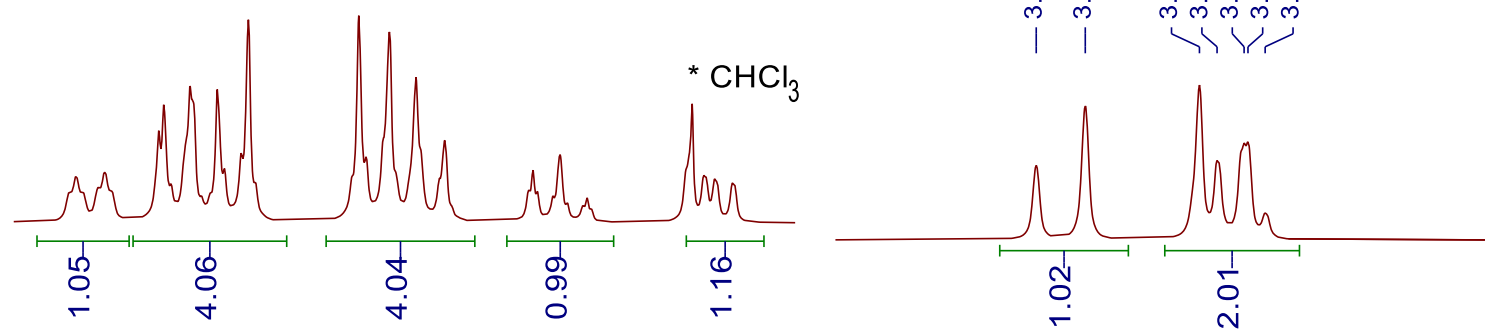

-

$\begin{array}{lllll}3.85 & 3.80 & 3.75 & 3.70 & 3.65\end{array}$

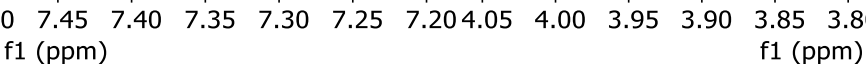

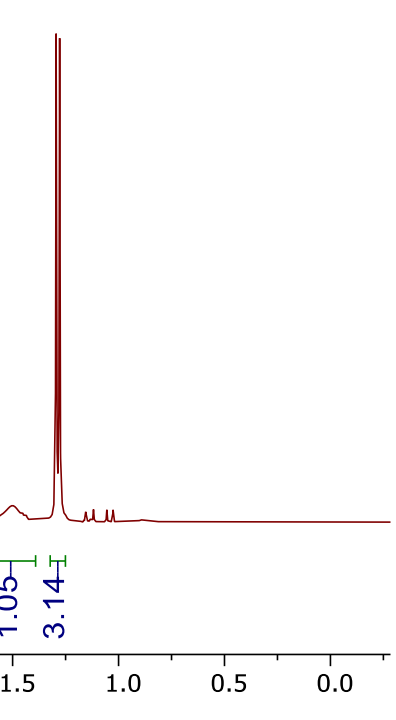


<smiles>C=C(c1ccc(-c2ccccc2)cc1)[C@H](C)NCc1cccnc1</smiles>

(R)-3af

${ }^{13} \mathrm{C}\left\{{ }^{1} \mathrm{H}\right\}$ NMR (100 MHz, $\left.\mathrm{CDCl}_{3}\right)$

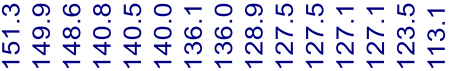

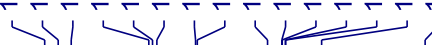

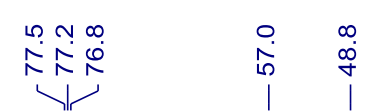

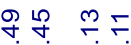

ind

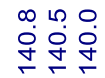

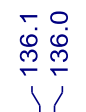

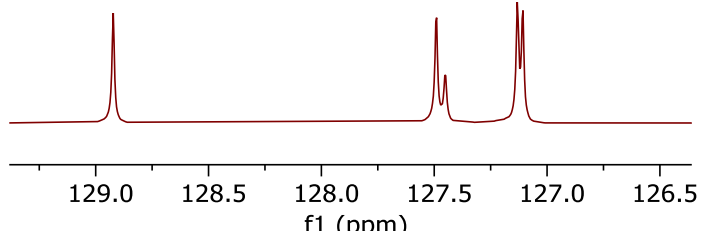

${ }^{*} \mathrm{CDCl}_{3}$
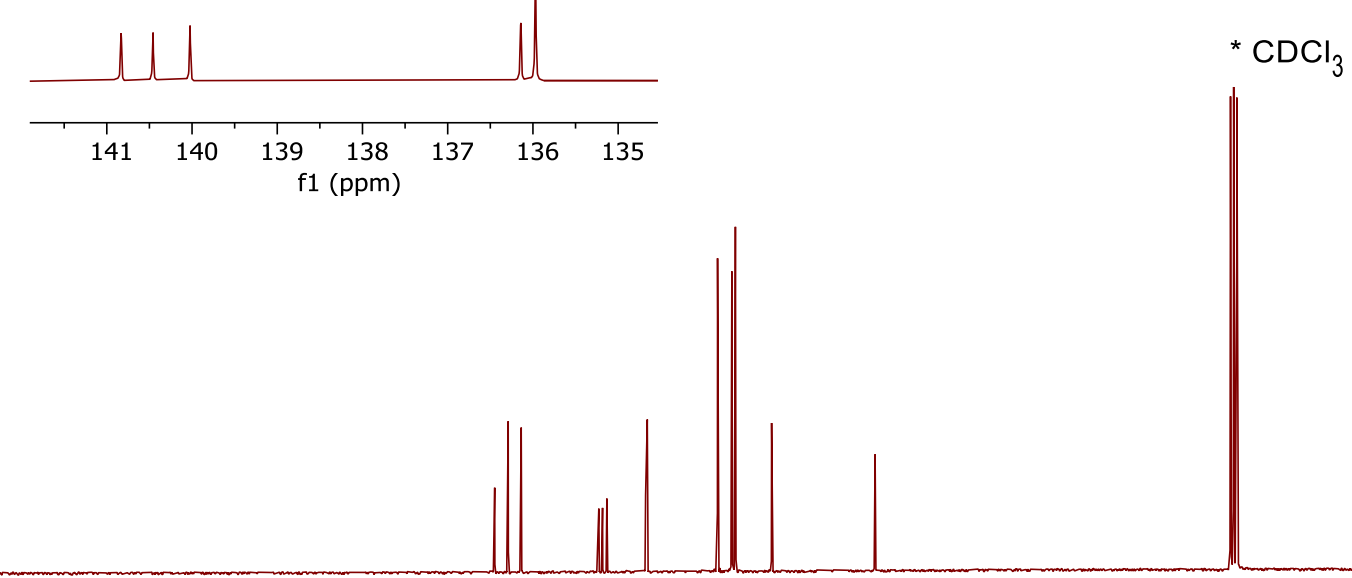

$210 \quad 200$ 


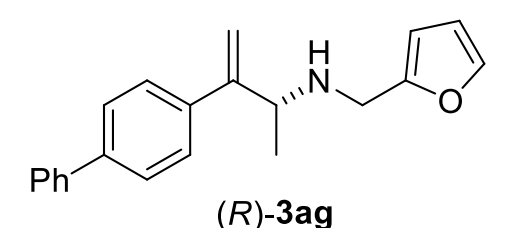

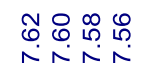

시순

$(R)$-3ag

${ }^{1} \mathrm{H}$ NMR $\left(400 \mathrm{MHz}, \mathrm{CDCl}_{3}\right)$
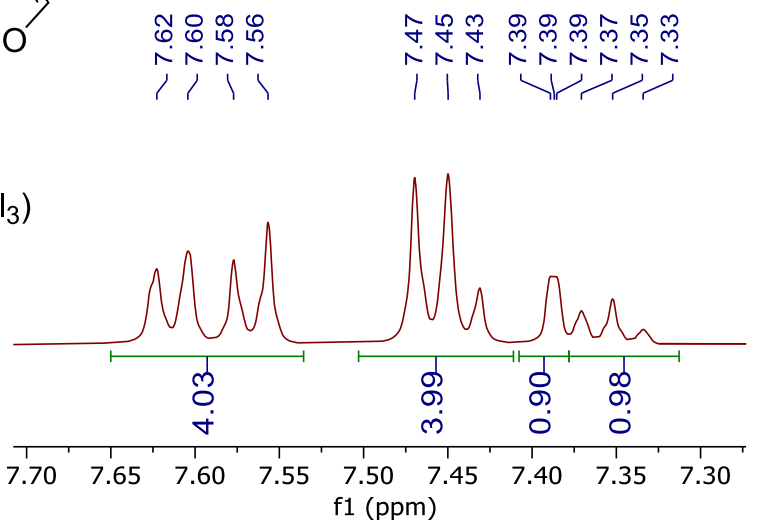

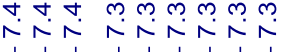

$\begin{array}{lll}1 & 1\end{array}$

IHNMR (400 MHz, CDC$$
\begin{array}{lllll}
7.70 & 7.65 & 7.60 & 7.55 & 7.50 \\
\mathrm{f} 1(\mathrm{ppm})
\end{array}
$$
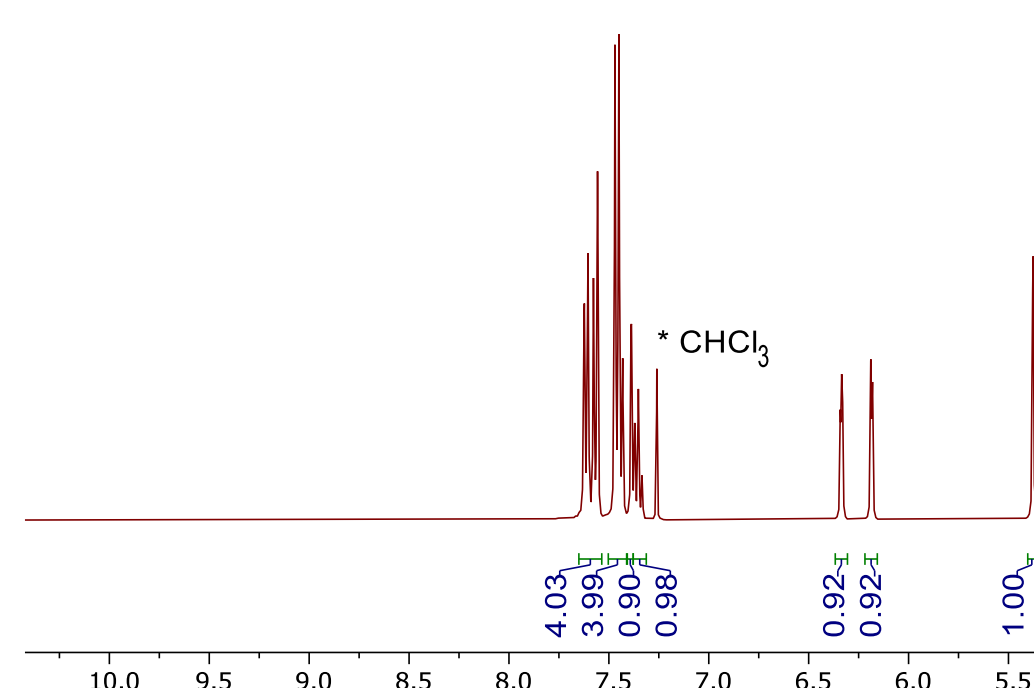$$
\text { (2) }
$$

$8.0 \quad 7.5 \quad 7.0$

$6.5 \quad 6.0$

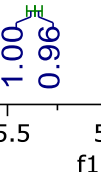

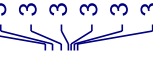
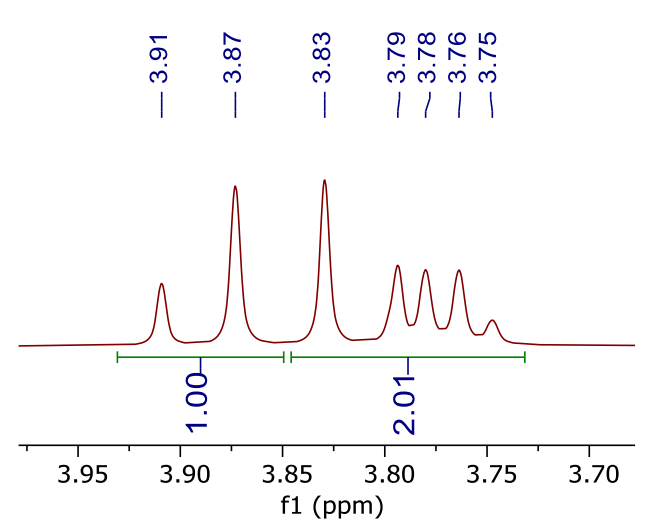

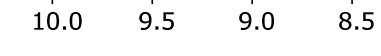

5.0
f1 (ppm) 

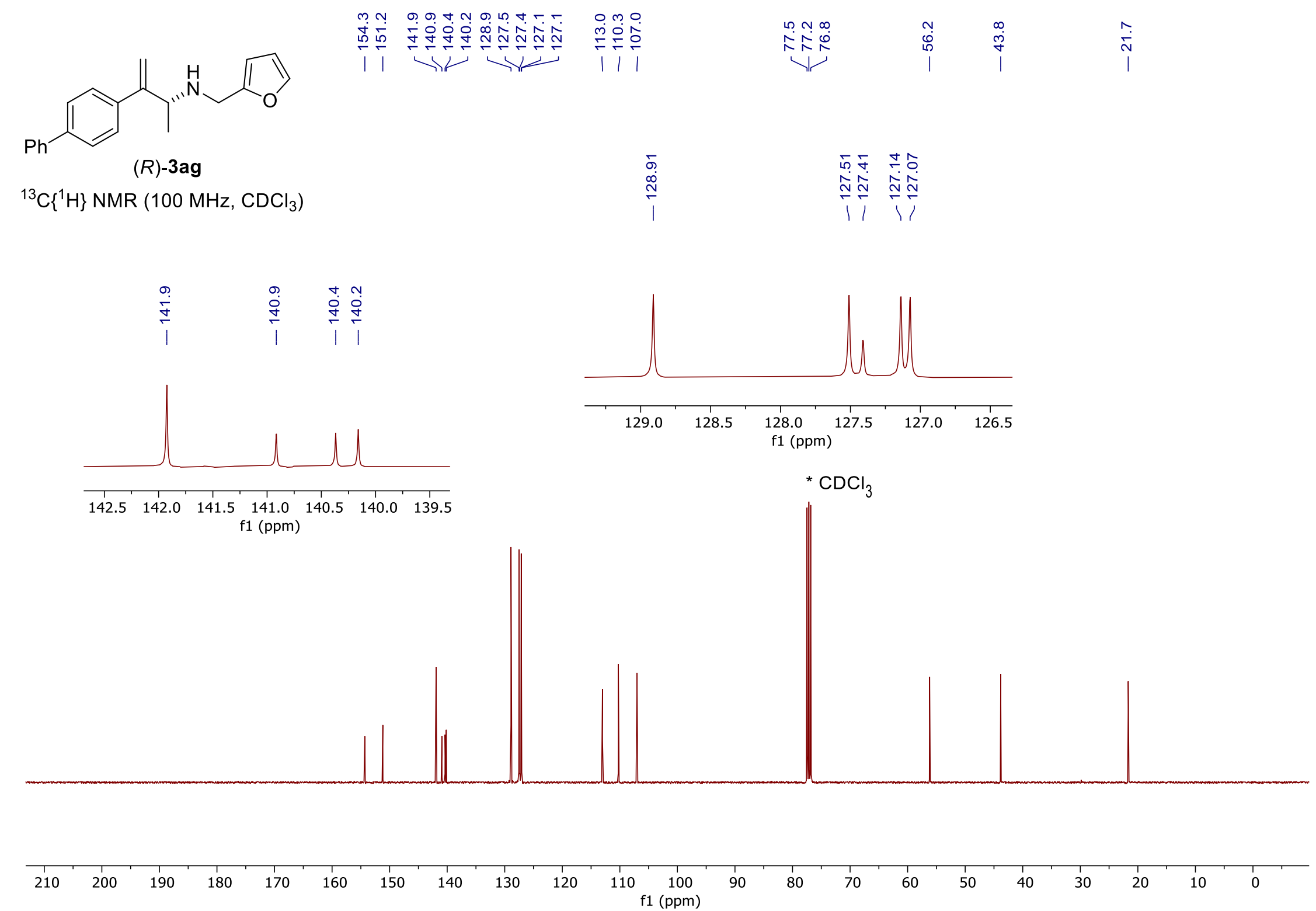


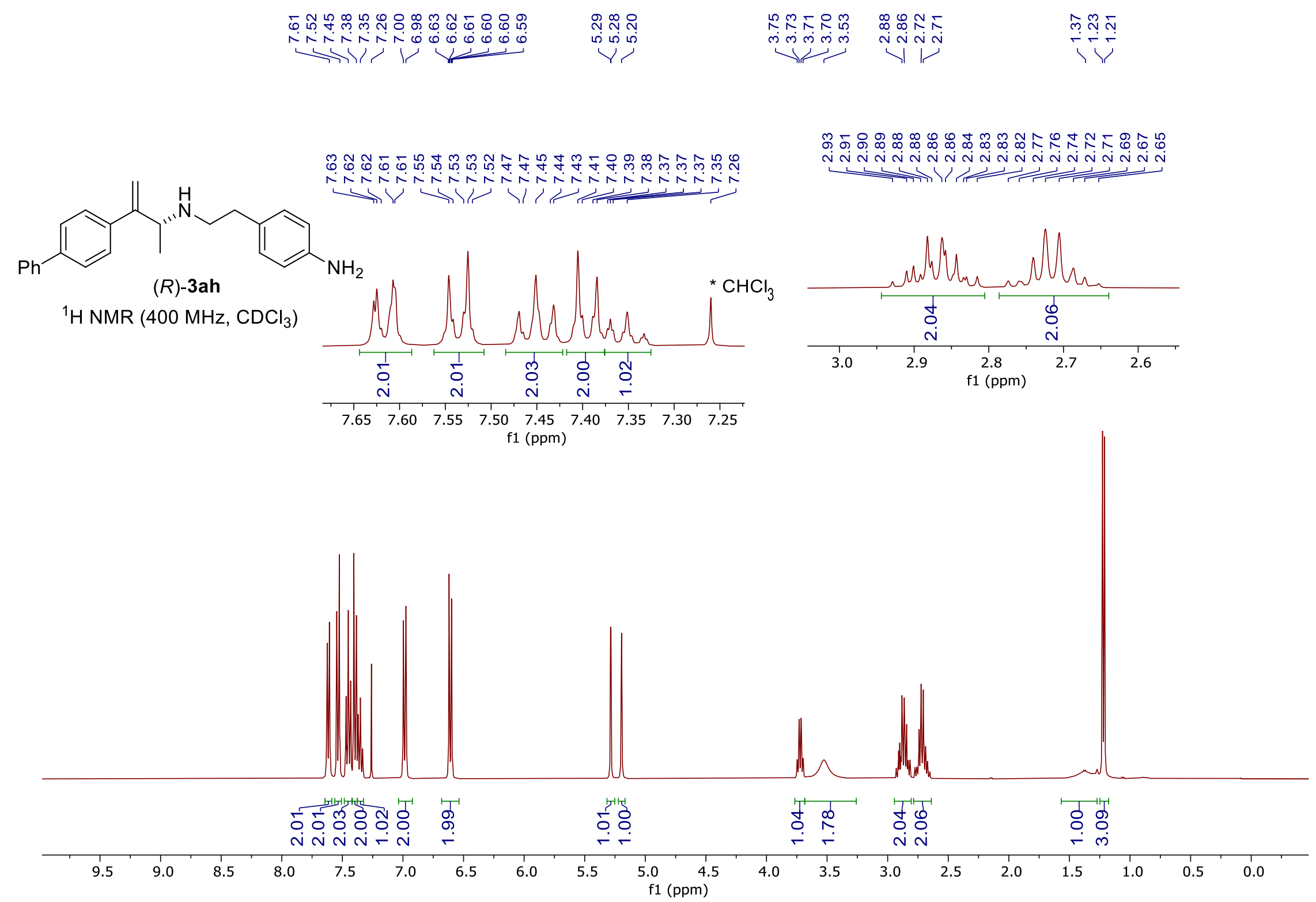




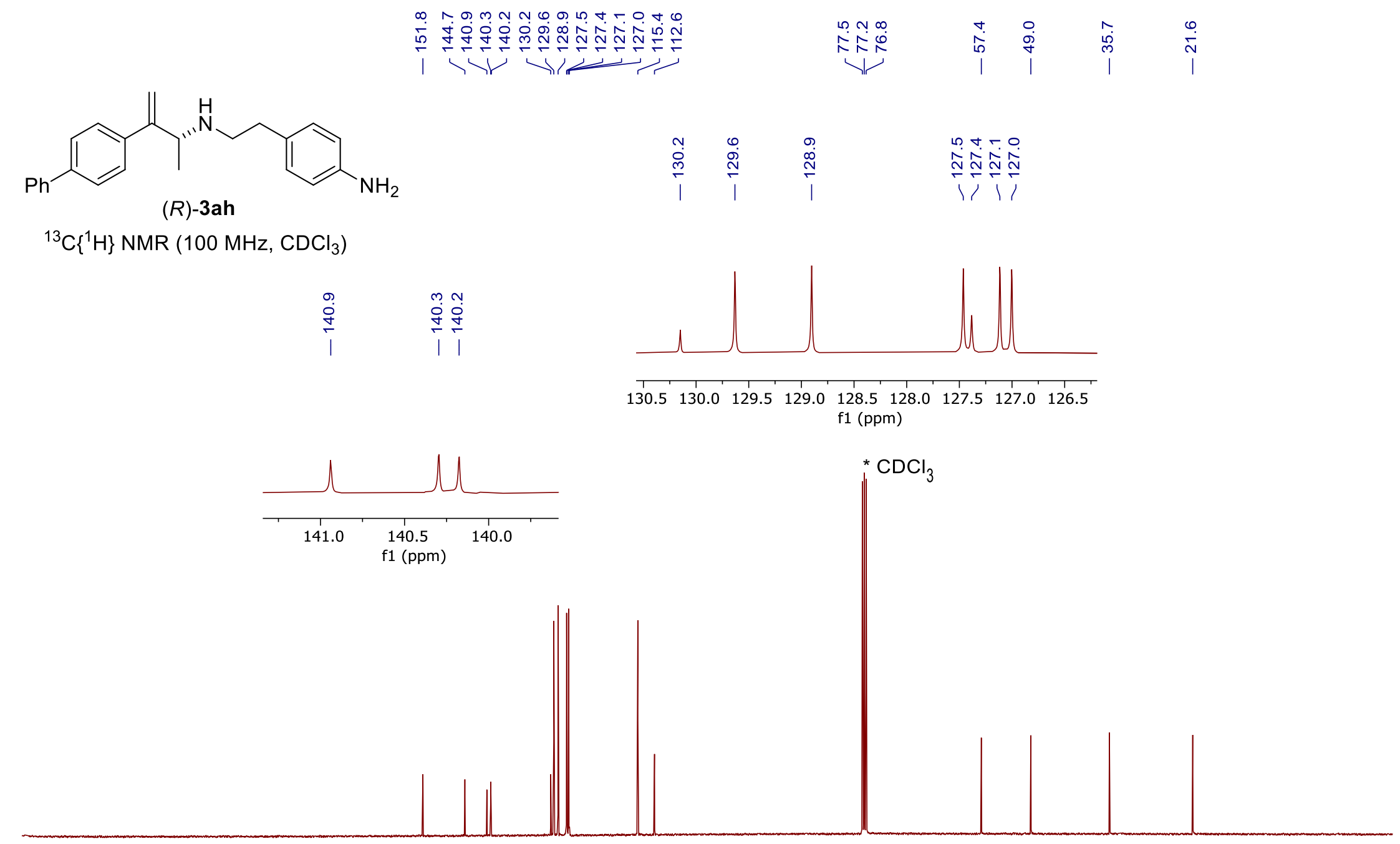

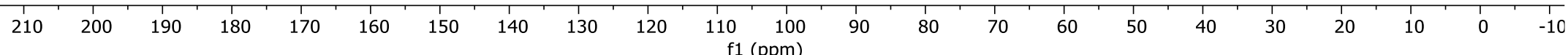




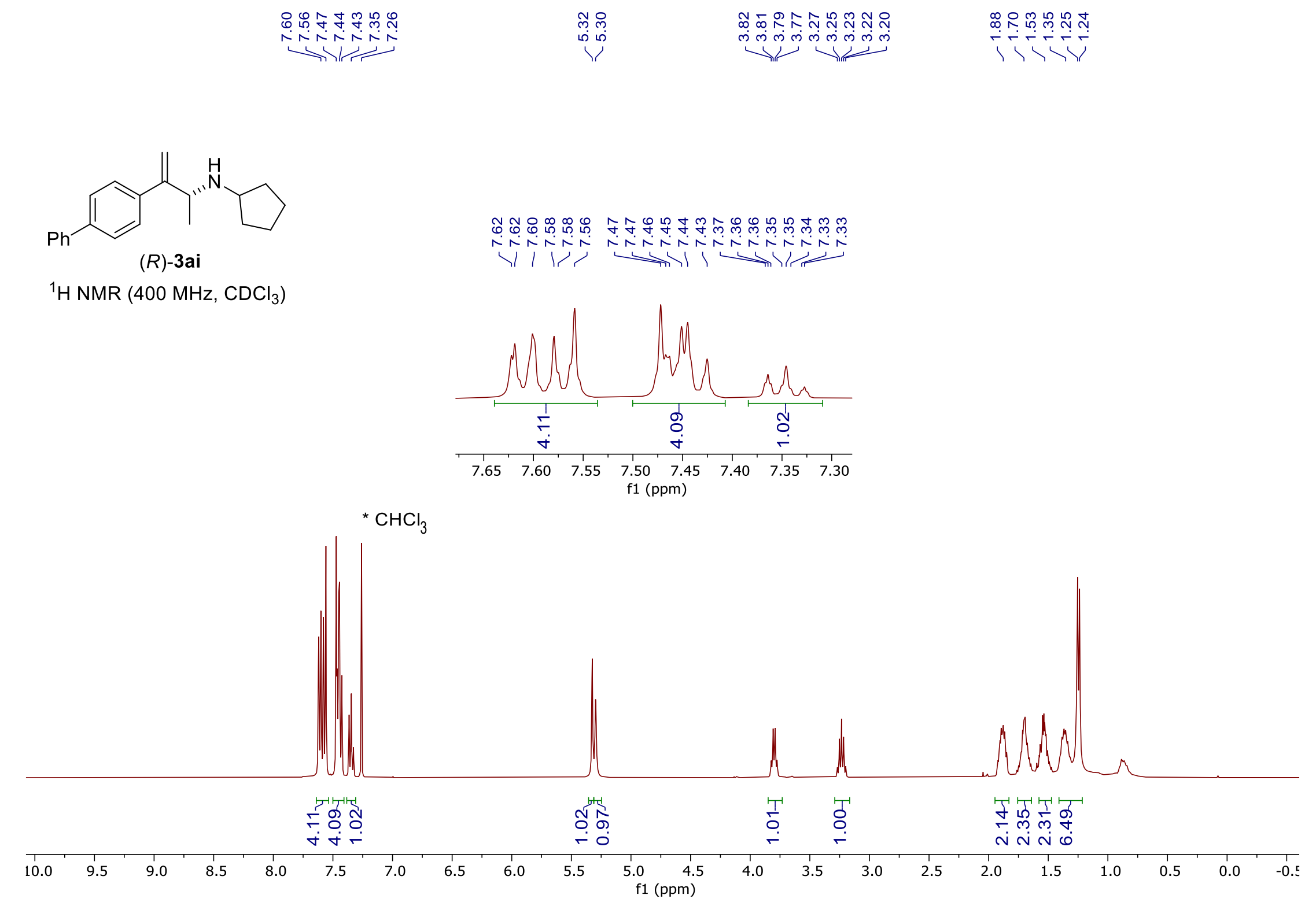




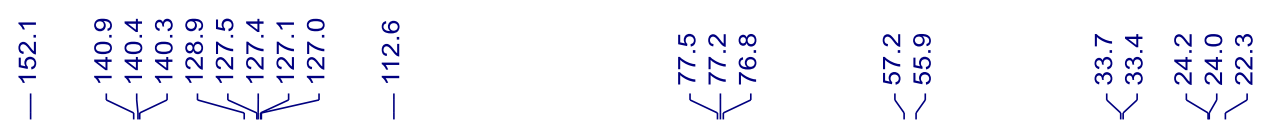

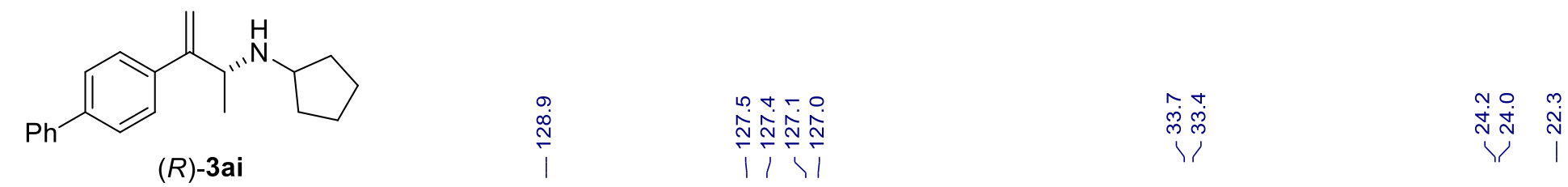

${ }^{13} \mathrm{C}\left\{{ }^{1} \mathrm{H}\right\} \mathrm{NMR}\left(100 \mathrm{MHz}, \mathrm{CDCl}_{3}\right)$
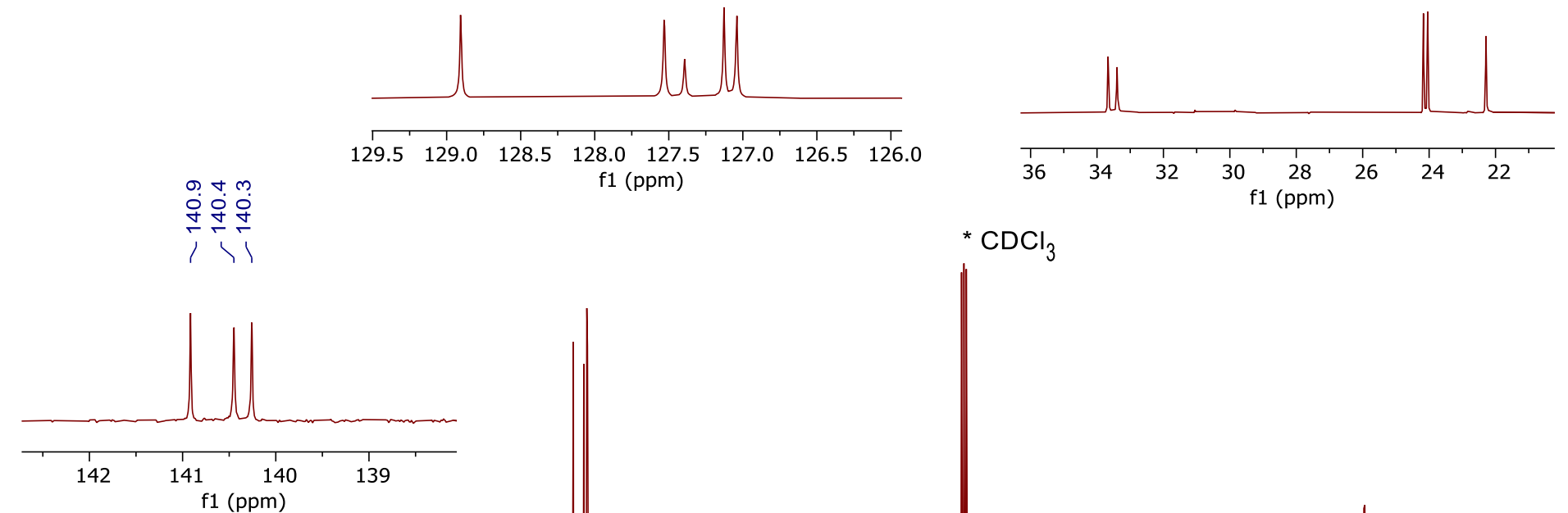

$\begin{array}{llllllllllllllllllllllllll}210 & 200 & 190 & 180 & 170 & 160 & 150 & 140 & 130 & 120 & 110 & 100 & 90 & 80 & 70 & 60 & 50 & 40 & 30 & 20 & 10 & 0 & -10 & 10\end{array}$ 


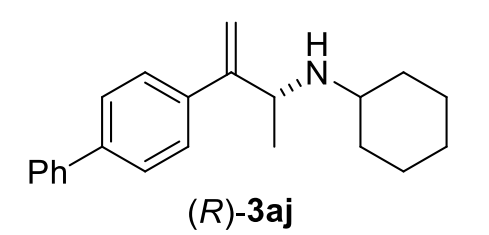
ivivis

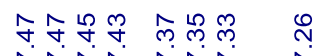

(R)-3aj

${ }^{1} \mathrm{H}$ NMR $\left(400 \mathrm{MHz}, \mathrm{CDCl}_{3}\right)$

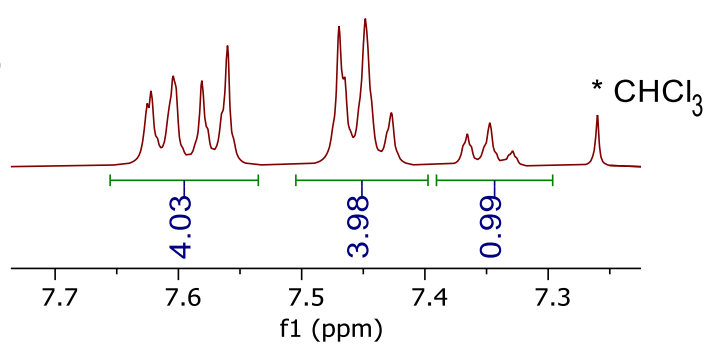

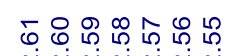

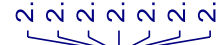

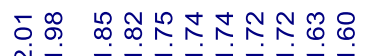

11 i

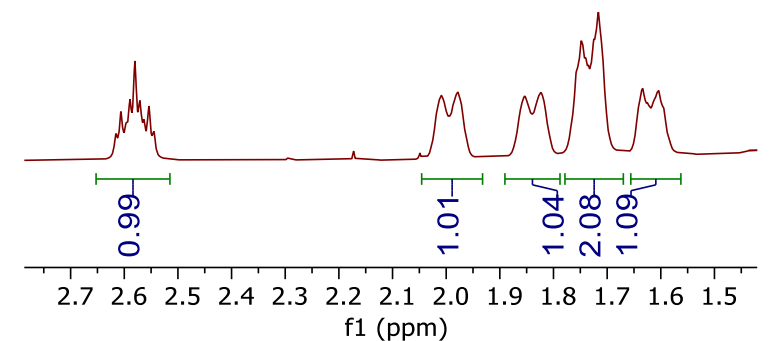

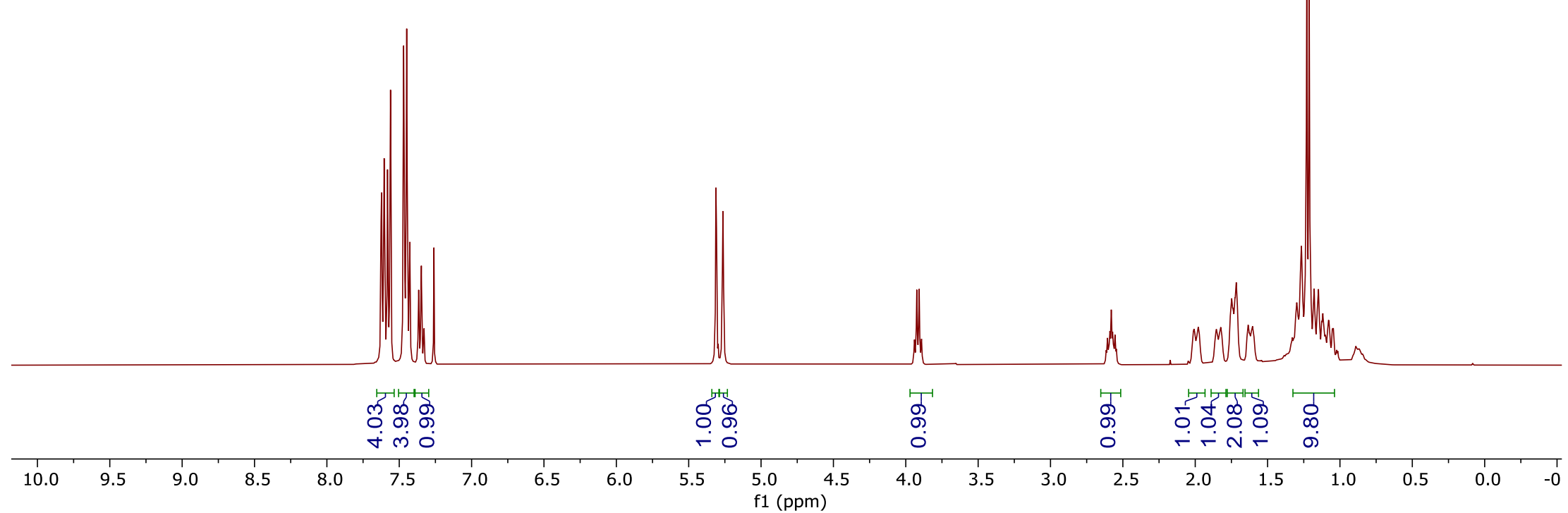


إل

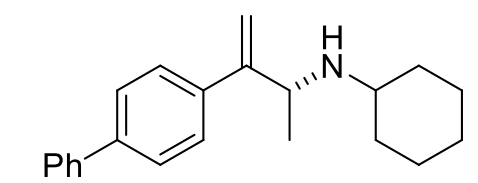

$(R)$-3aj

${ }^{13} \mathrm{C}\left\{{ }^{1} \mathrm{H}\right\} \mathrm{NMR}\left(100 \mathrm{MHz}, \mathrm{CDCl}_{3}\right)$
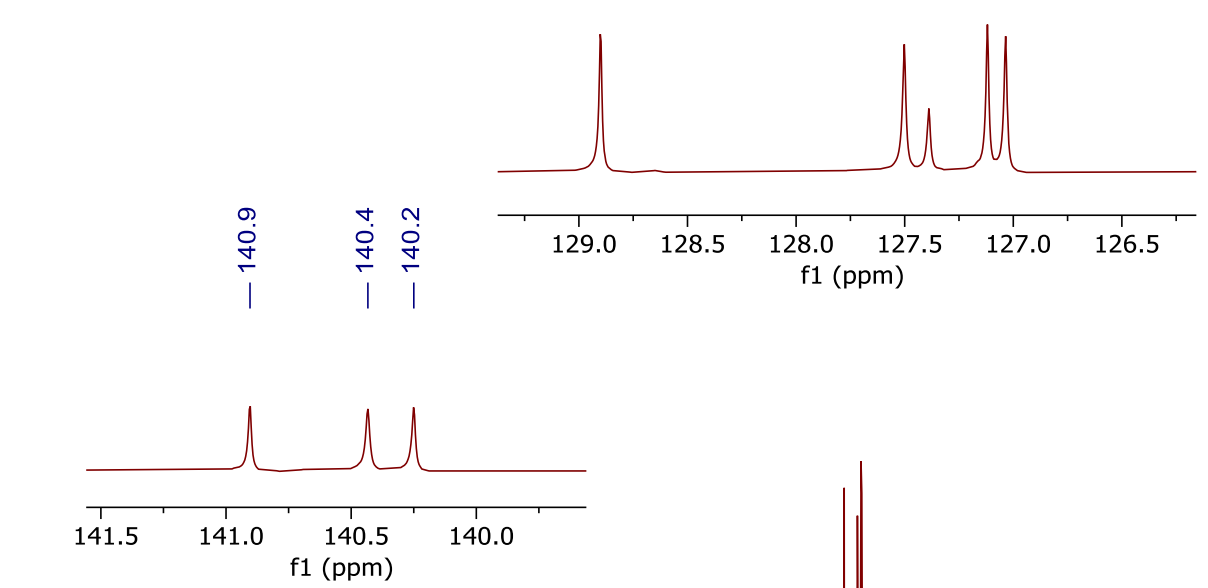

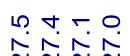

$\operatorname{lin}\left(\frac{1}{1}\right.$

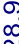

$\stackrel{\infty}{\sim}$

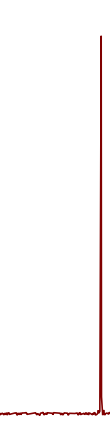

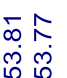

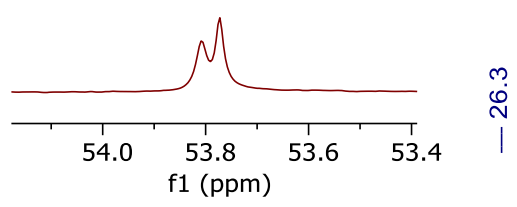

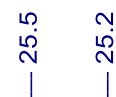

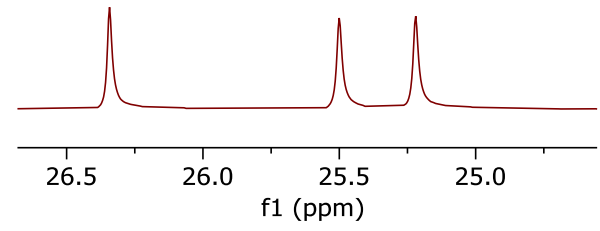

${ }^{*} \mathrm{CDCl}_{3}$

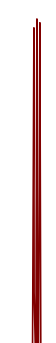




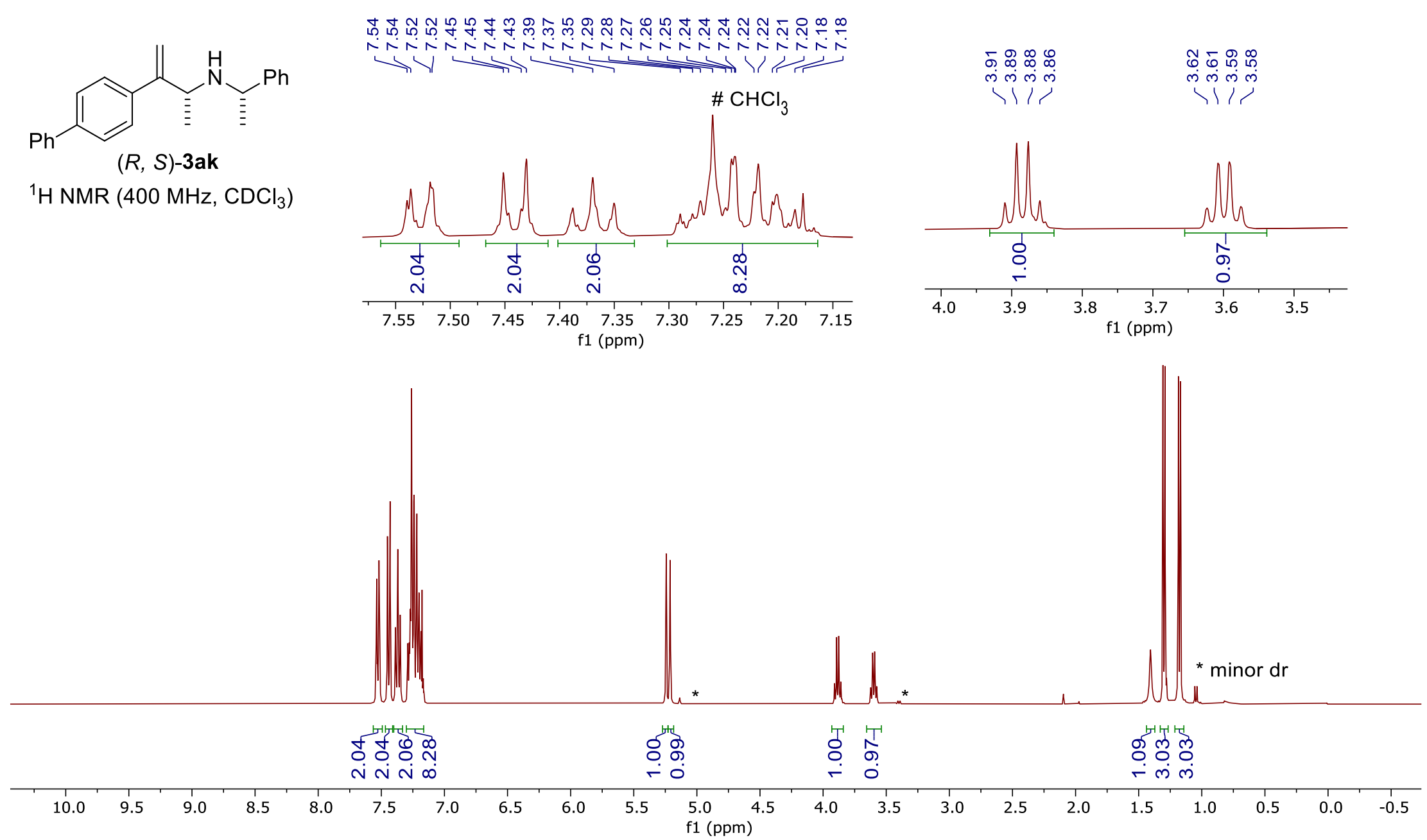


$\underbrace{\dot{j}}$

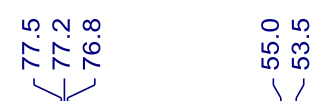

$\stackrel{\circ}{\dot{*}} \stackrel{0}{\stackrel{1}{N}}$

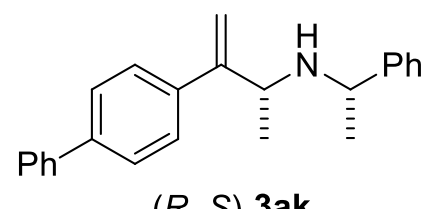

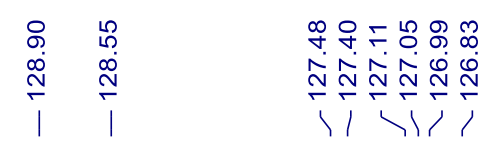

${ }^{13} \mathrm{C}\left\{{ }^{1} \mathrm{H}\right\}$ NMR $\left(100 \mathrm{MHz}, \mathrm{CDCl}_{3}\right)$
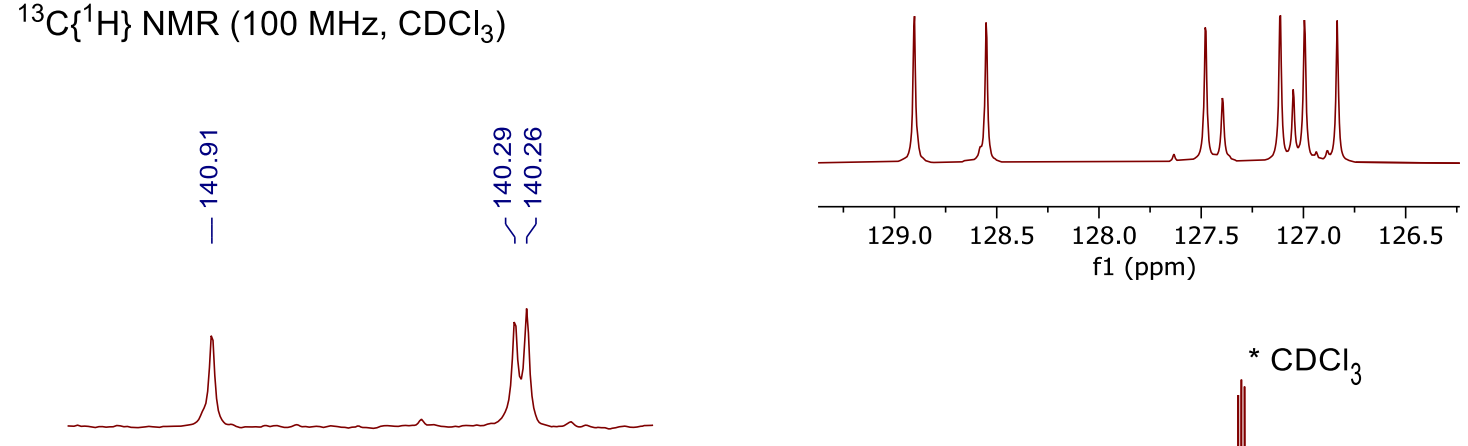

$\begin{array}{llllll}141.2 & 141.0 & 140.8 & 140.6 & 140.4 & 140.2\end{array}$ f1 (ppm)
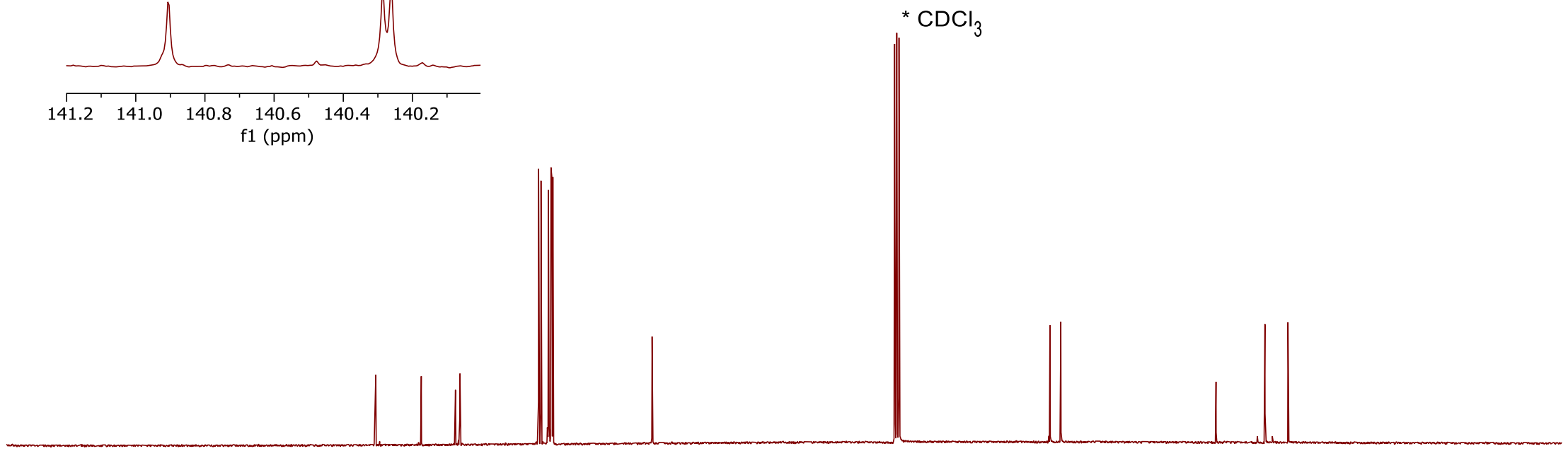

200

190

$180 \quad 170$

$160 \quad 150$

140

$130 \quad 120$

$\begin{array}{lll}110 & 100 & 90\end{array}$

f1 (ppm) 


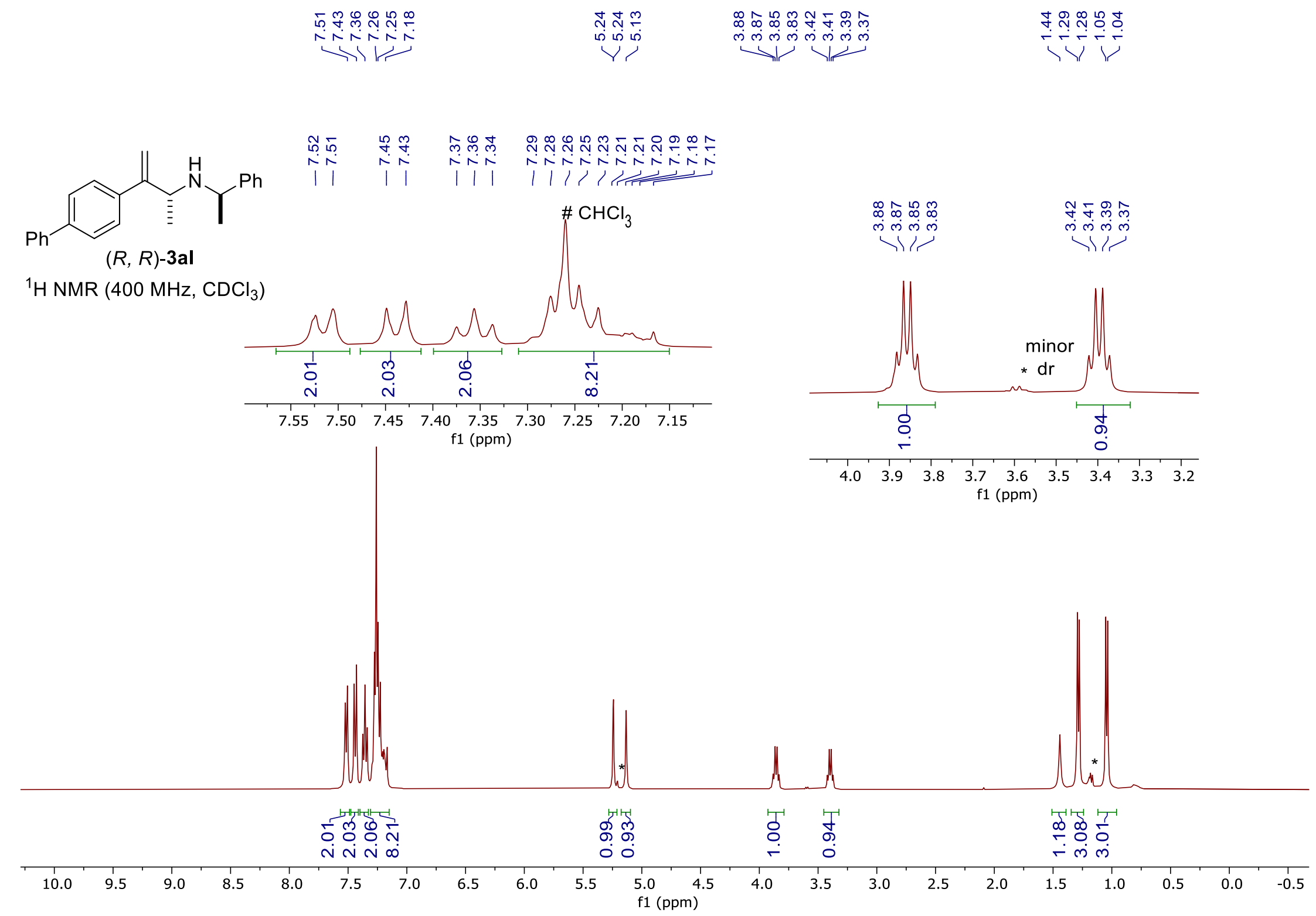




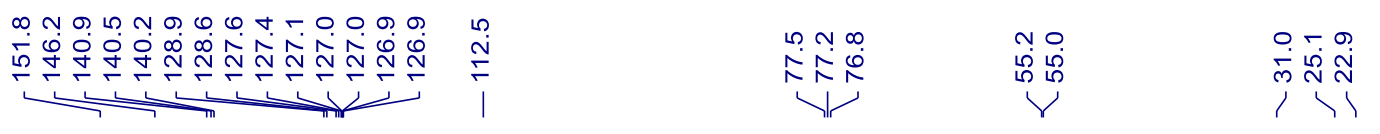

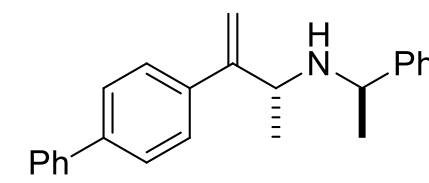

$(R, R)$-3al

${ }^{13} \mathrm{C}\left\{{ }^{1} \mathrm{H}\right\} \mathrm{NMR}\left(100 \mathrm{MHz}, \mathrm{CDCl}_{3}\right)$
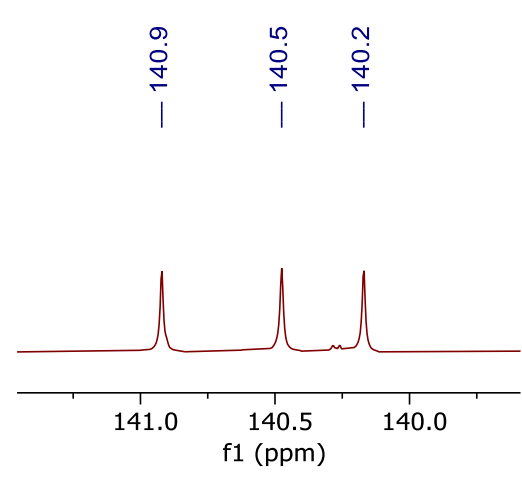

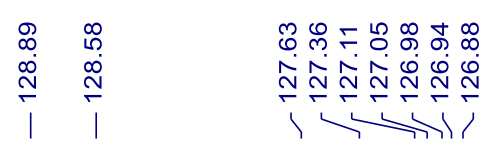

i
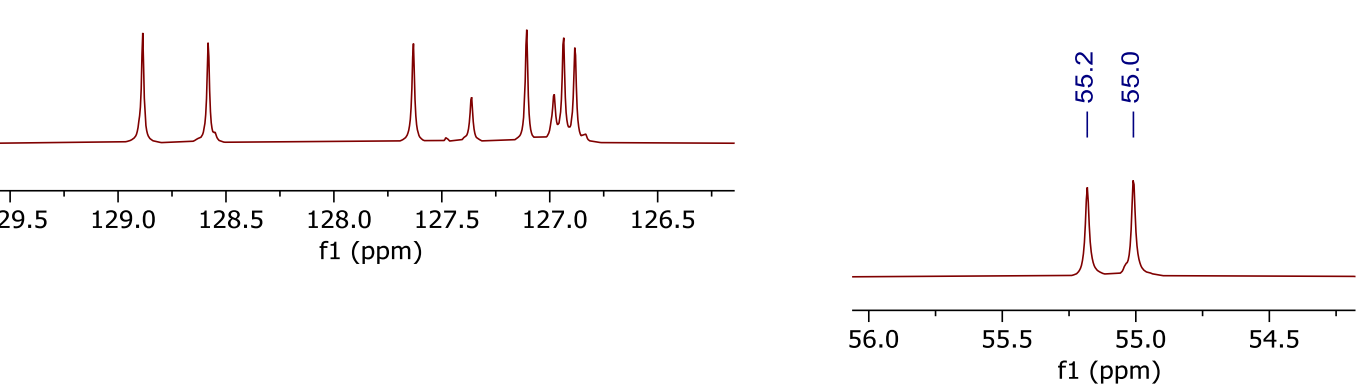

${ }^{*} \mathrm{CDCl}_{3}$
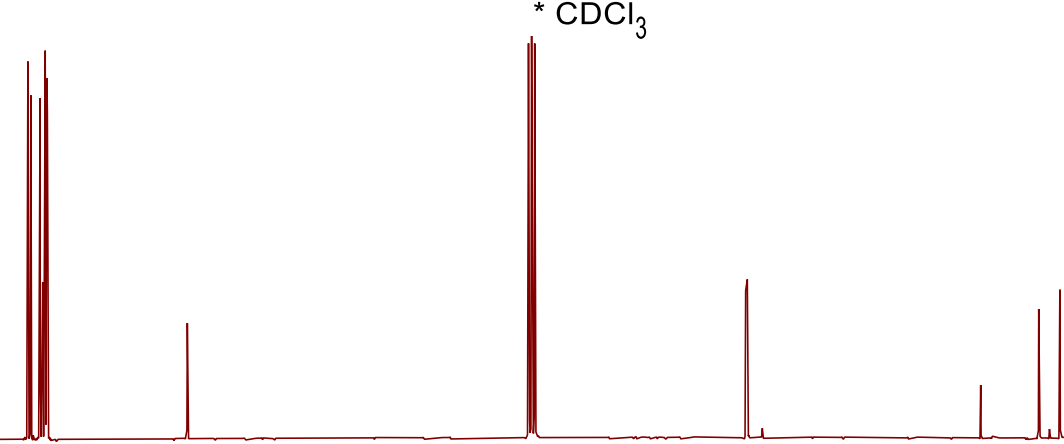


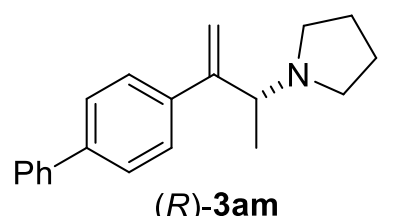

${ }^{1} \mathrm{H}$ NMR (400 MHz, $\mathrm{CDCl}_{3}$ )

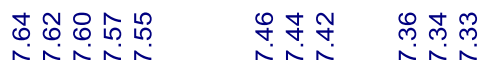

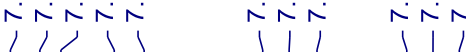

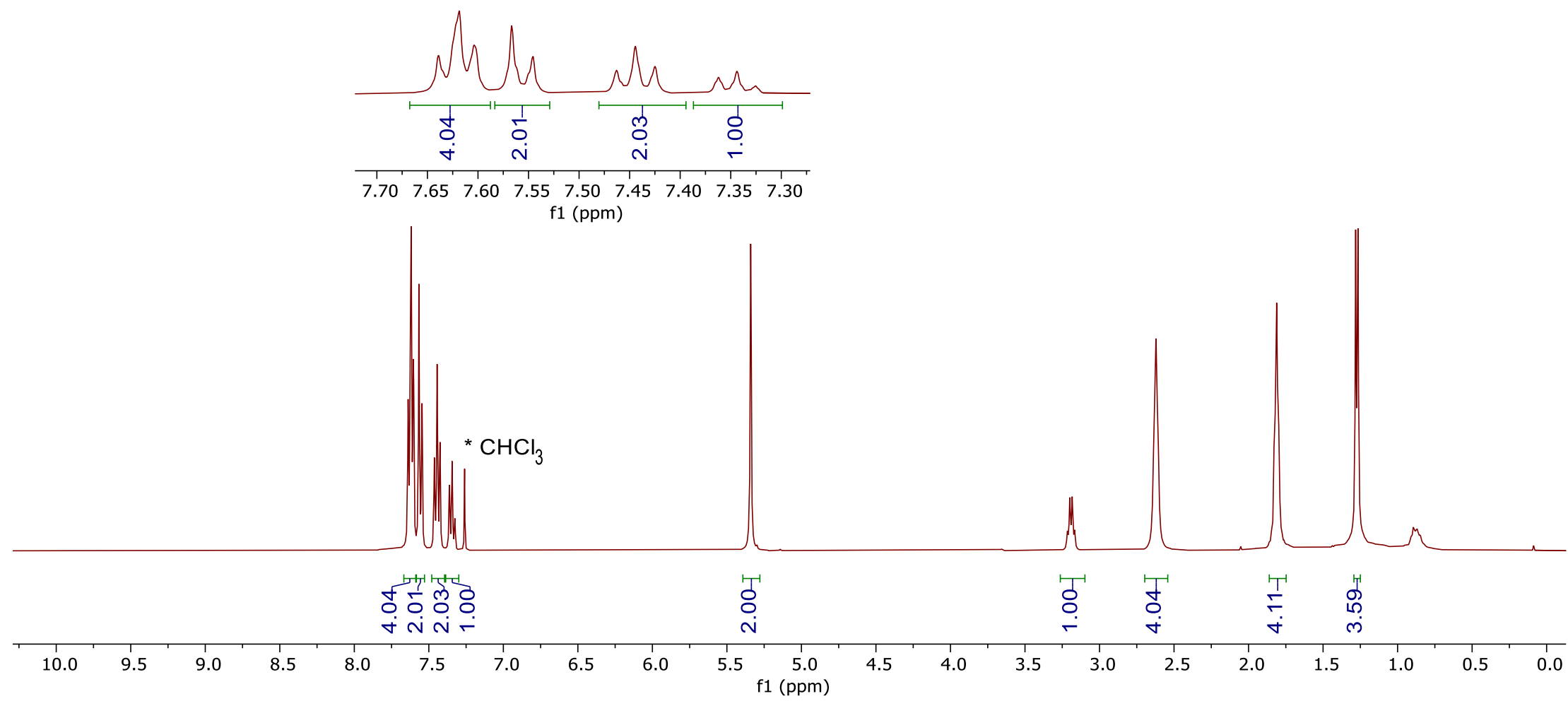




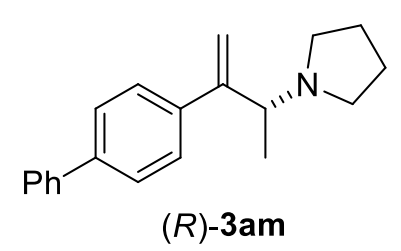

${ }^{13} \mathrm{C}\left\{{ }^{1} \mathrm{H}\right\}$ NMR (100 MHz, $\mathrm{CDCl}_{3}$ )

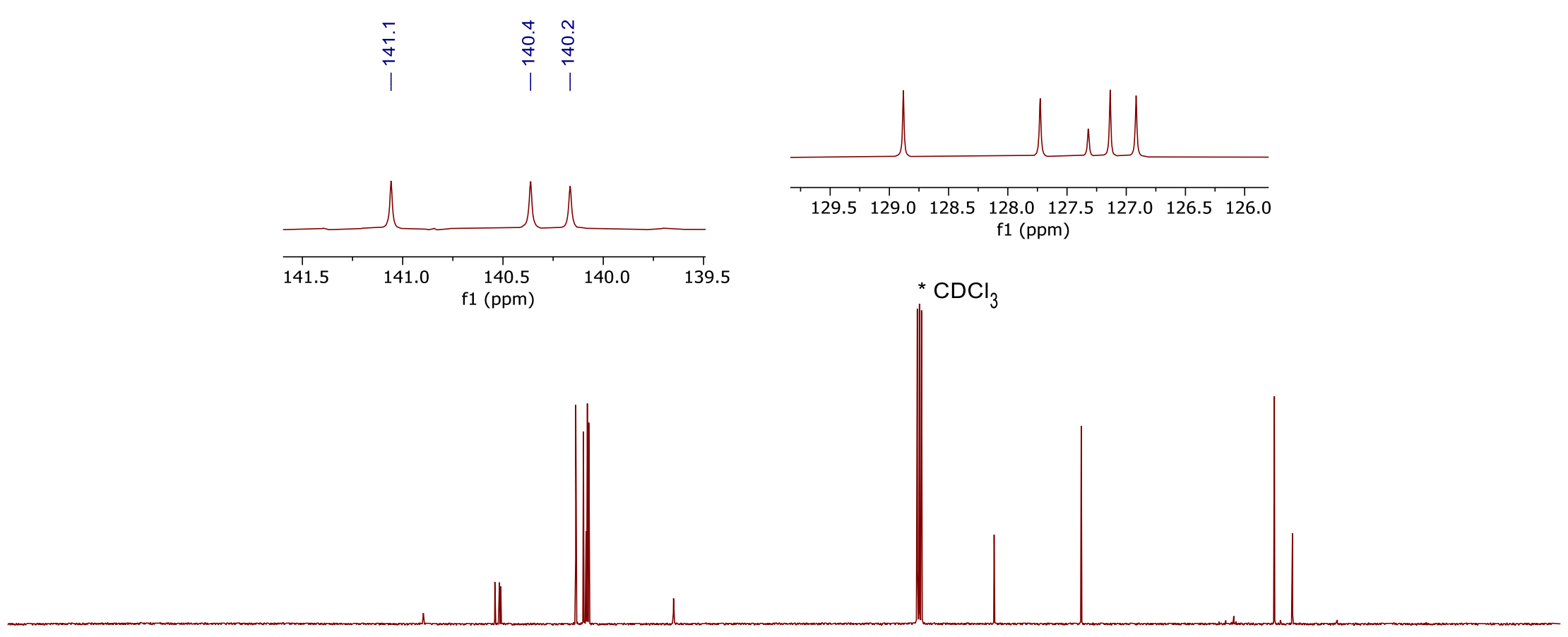

$\underbrace{\infty}_{\frac{1}{2}}$ 


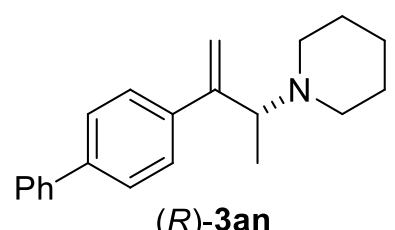

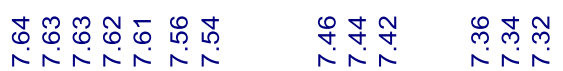

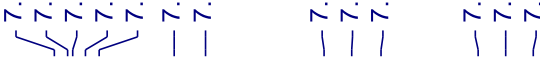

${ }^{1} \mathrm{H}$ NMR $\left(400 \mathrm{MHz}, \mathrm{CDCl}_{3}\right)$

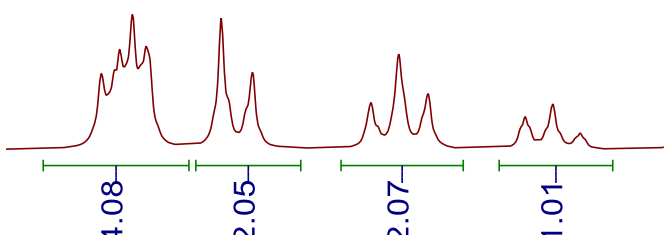

$\begin{array}{lllllllllll}7.70 & 7.65 & 7.60 & 7.55 & 7.50 & 7.45 & 7.40 & 7.35 & 7.30\end{array}$

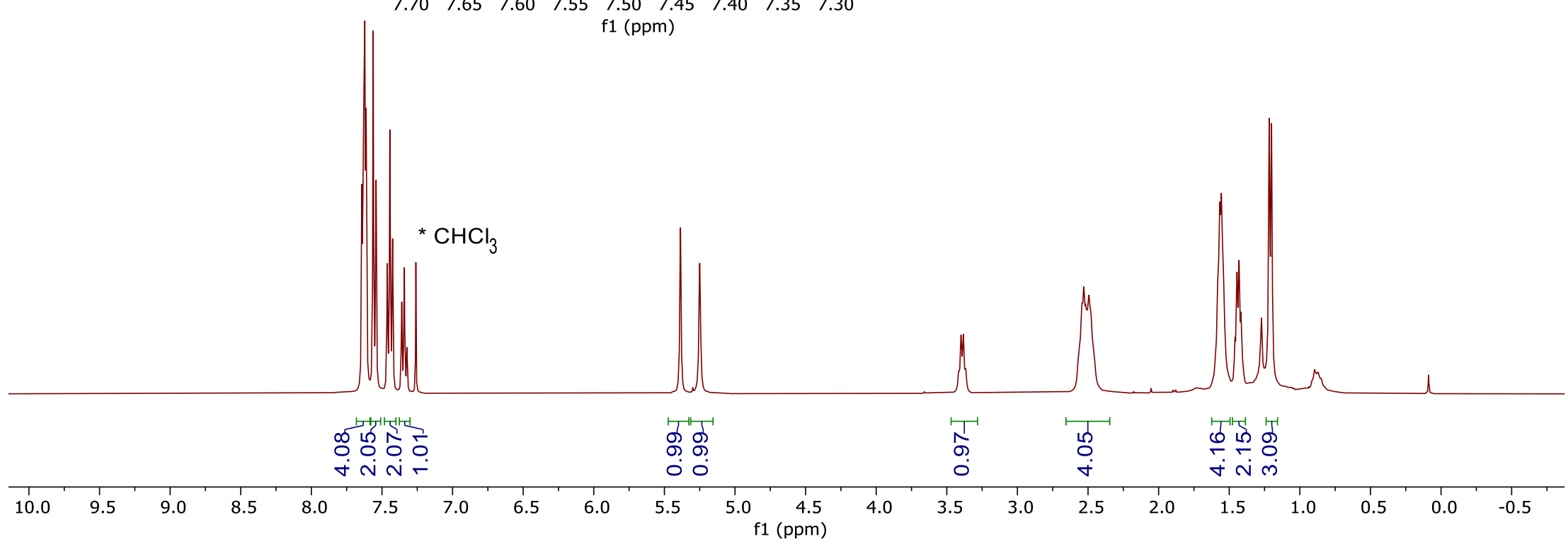




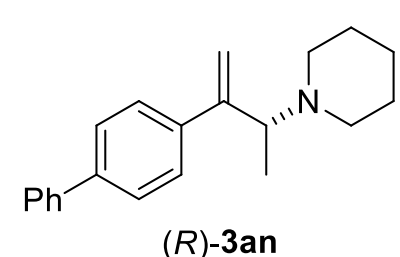

${ }^{13} \mathrm{C}\left\{{ }^{1} \mathrm{H}\right\} \operatorname{NMR}\left(100 \mathrm{MHz}, \mathrm{CDCl}_{3}\right)$

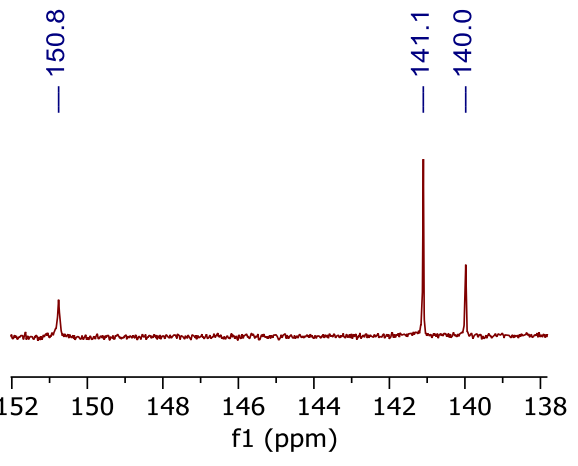

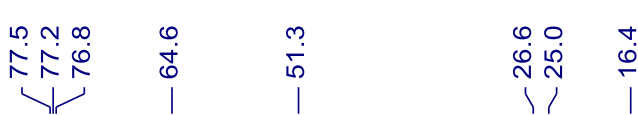

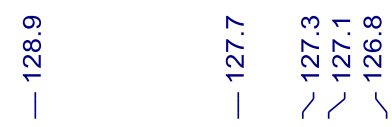

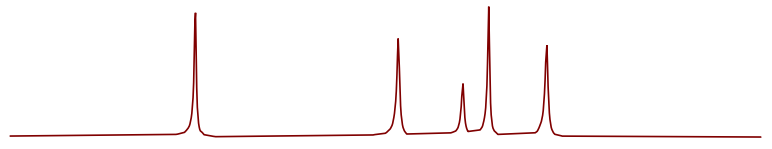

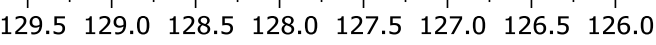
f1 (ppm)

${ }^{*} \mathrm{CDCl}_{3}$ 


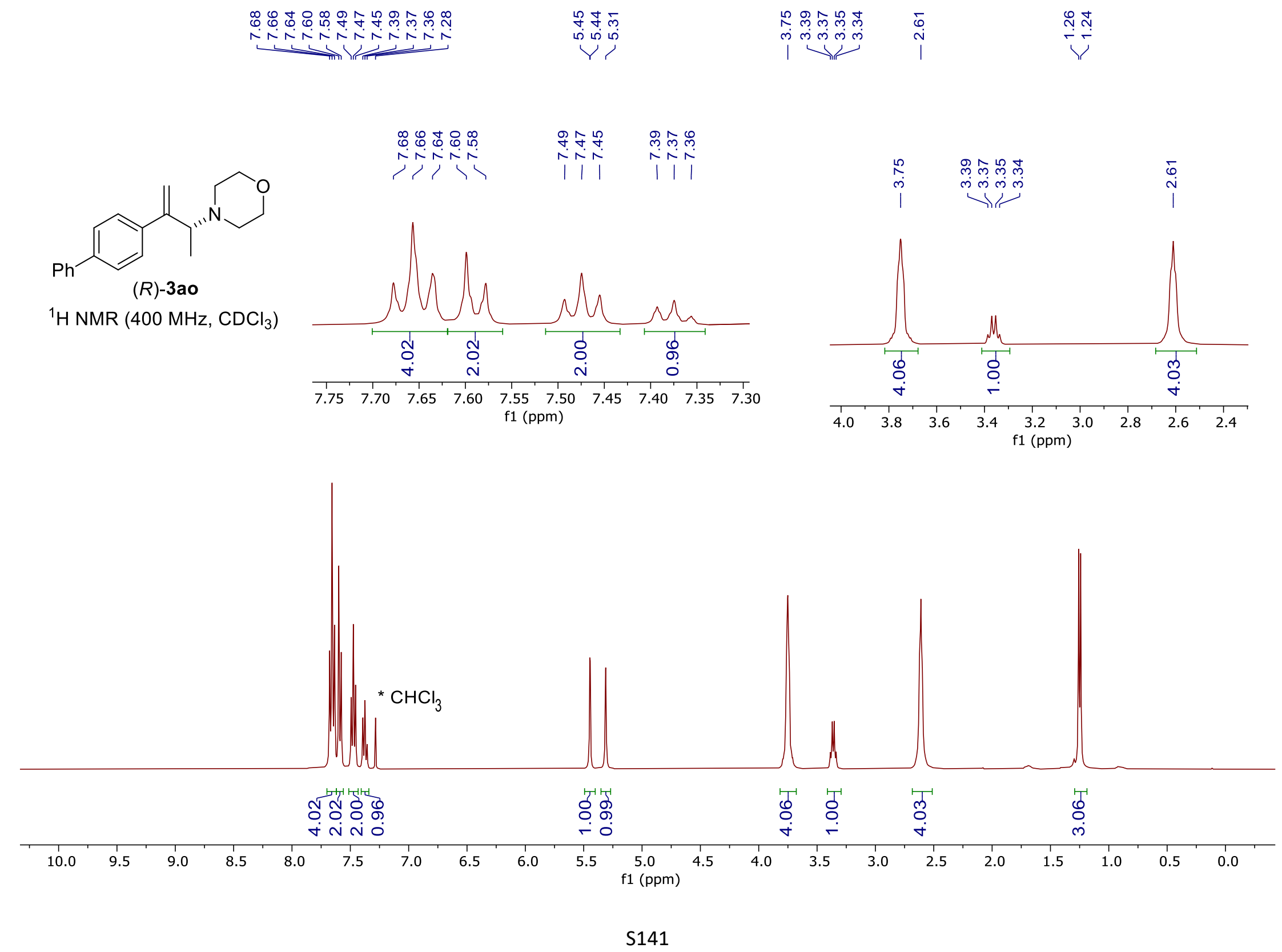




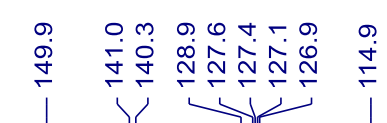

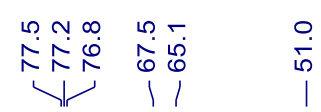

$\stackrel{\infty}{\stackrel{\infty}{+}}$

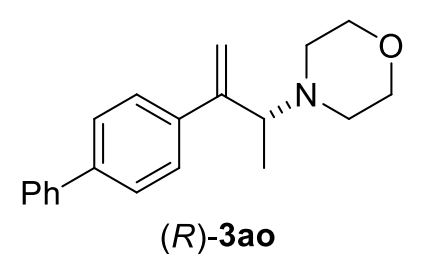

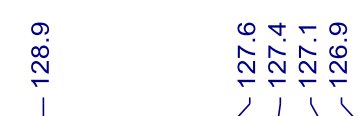

${ }^{13} \mathrm{C}\left\{{ }^{1} \mathrm{H}\right\}$ NMR $\left(100 \mathrm{MHz}, \mathrm{CDCl}_{3}\right)$

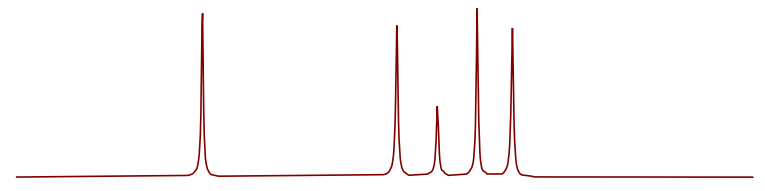

$\begin{array}{lllllllllllll}130.0 & 129.5 & 129.0 & 128.5 & 128.0 & 127.5 & 127.0 & 126.5 & 126.0 & 125.5\end{array}$ f1 (ppm)

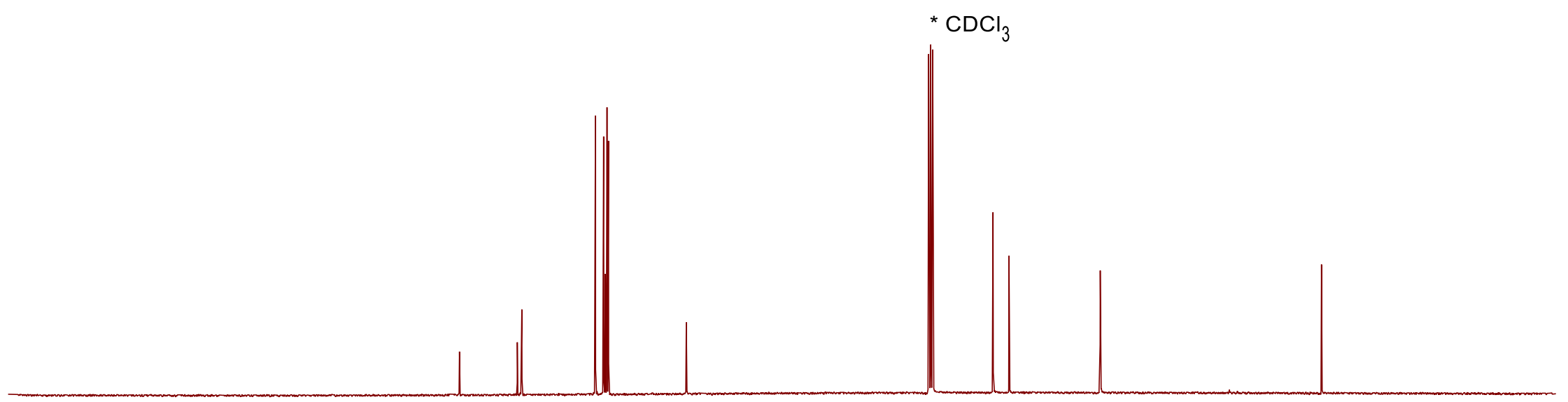

$\begin{array}{llllll}210 & 200 & 190 & 180 & 170 & 160\end{array}$

$150 \quad 140$

$130 \quad 120$

$110 \quad 100$

f1 (ppm) 


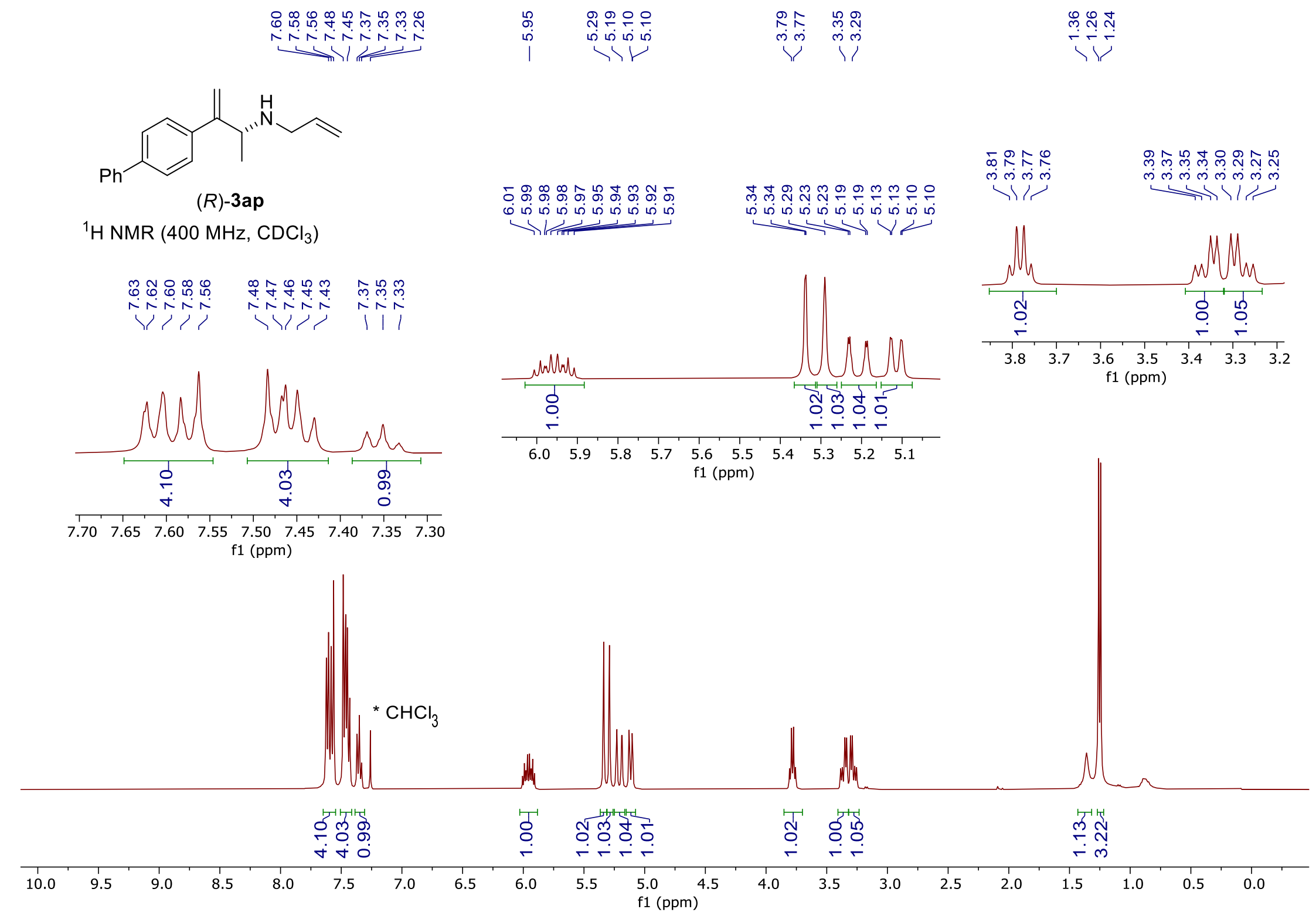




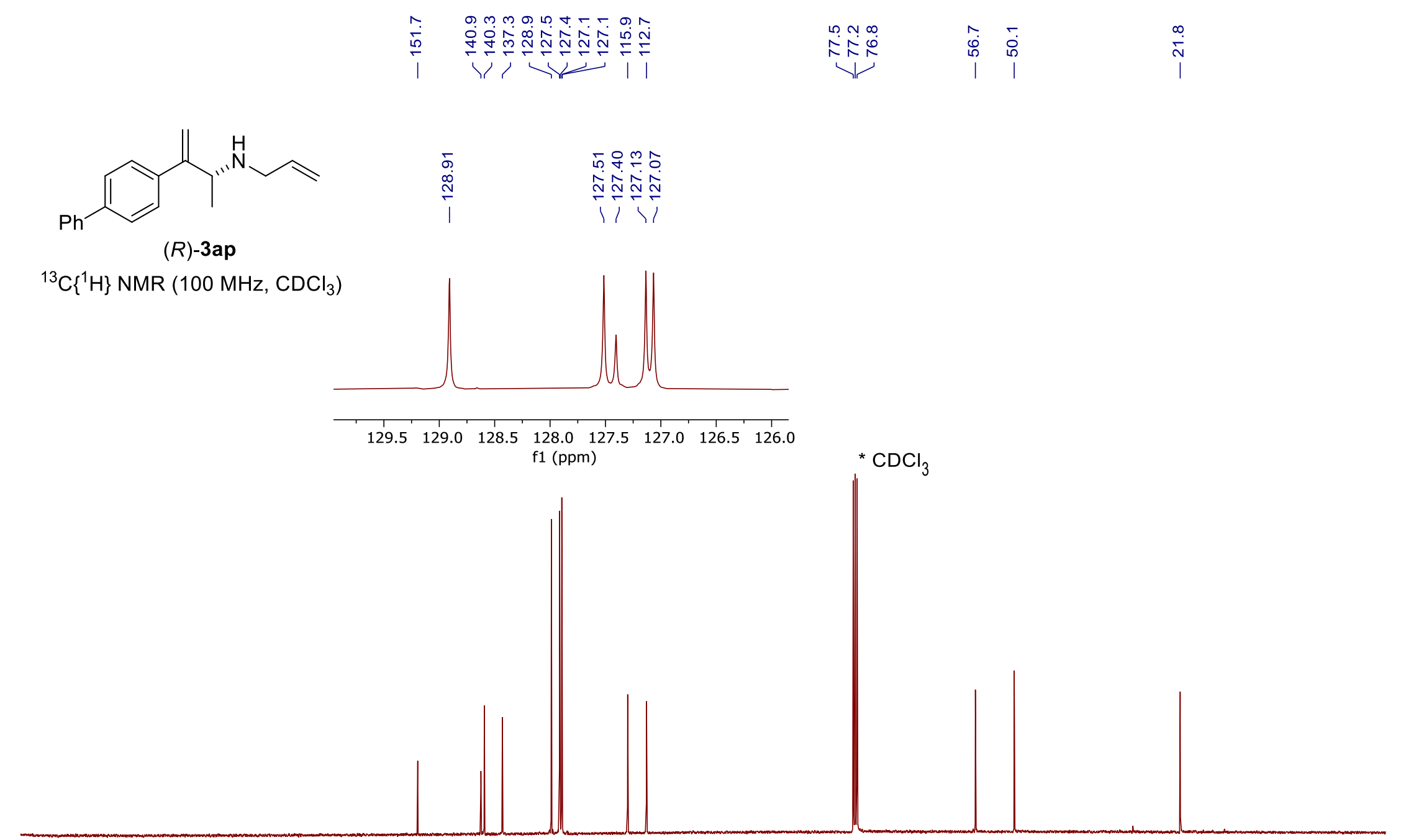

C\{ $\left.{ }^{1} \mathrm{H}\right\}$ NMR (100 MHz, C

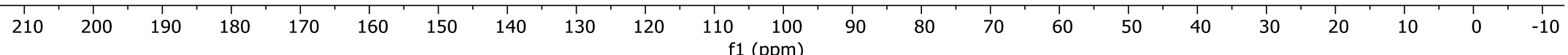




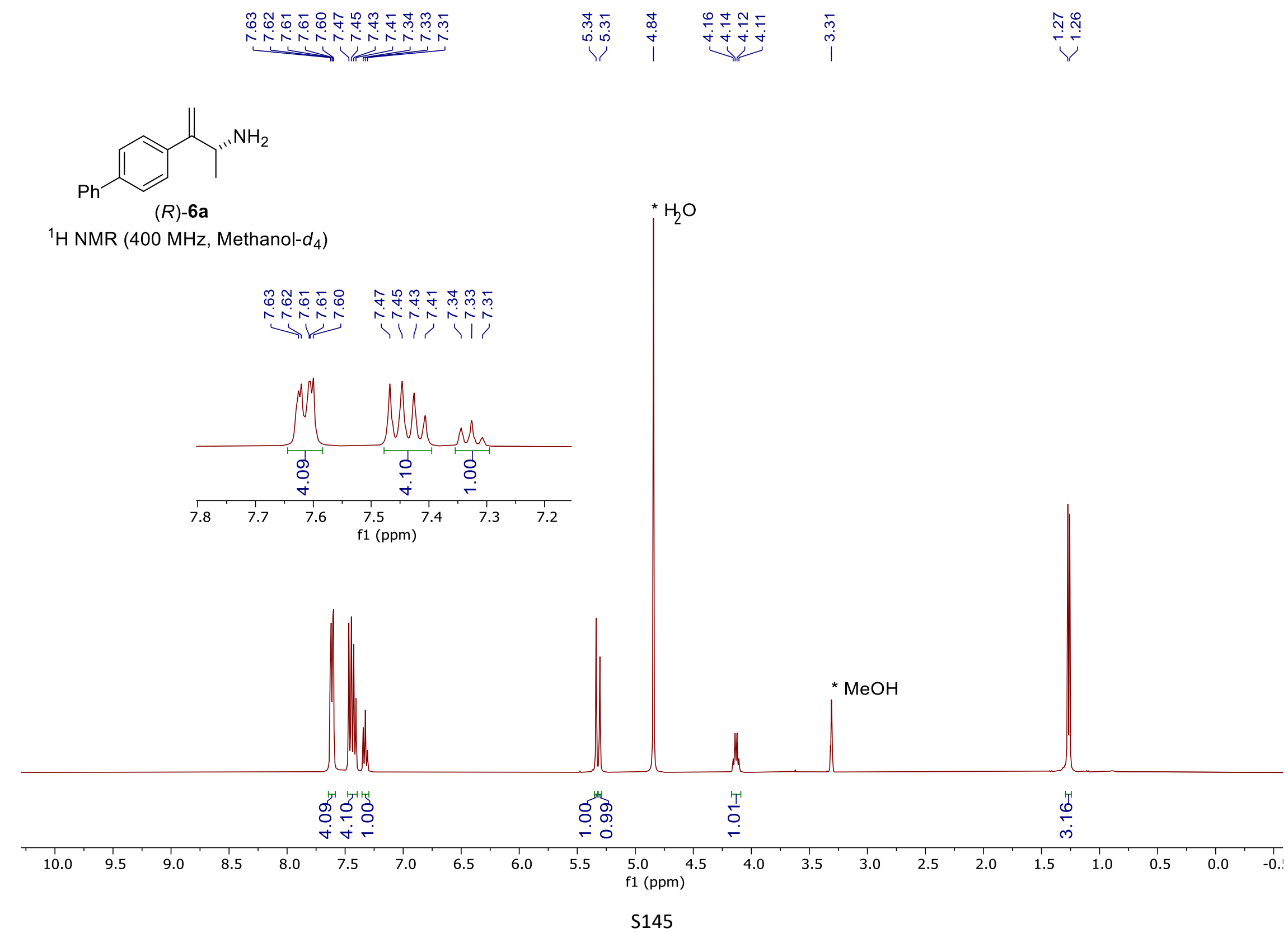




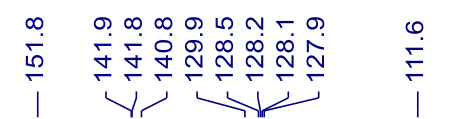<smiles>C=C(c1ccc(-c2ccccc2)cc1)[C@H](C)N</smiles>

(R)-6a

${ }^{13} \mathrm{C}\left\{{ }^{1} \mathrm{H}\right\}$ NMR $\left(100 \mathrm{MHz}\right.$, Methanol- $\left.d_{4}\right)$

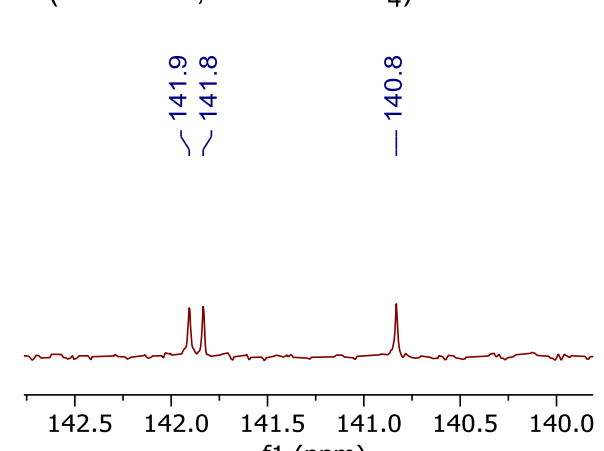

f1 (ppm)

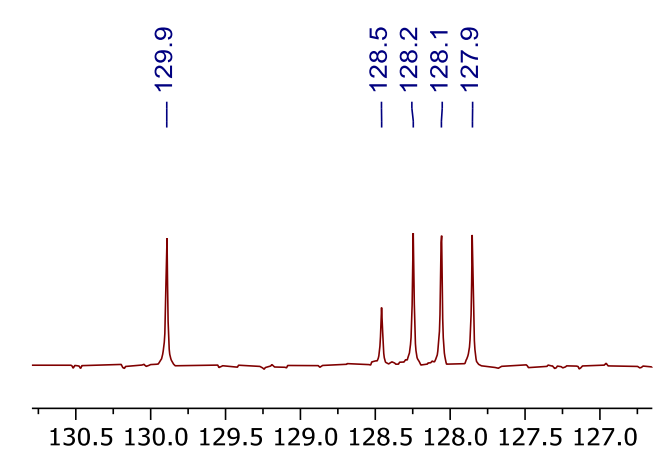

f1 (ppm)

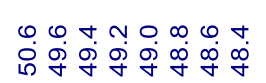

ลั่
${ }^{*} \mathrm{CD}_{3} \mathrm{OD}$

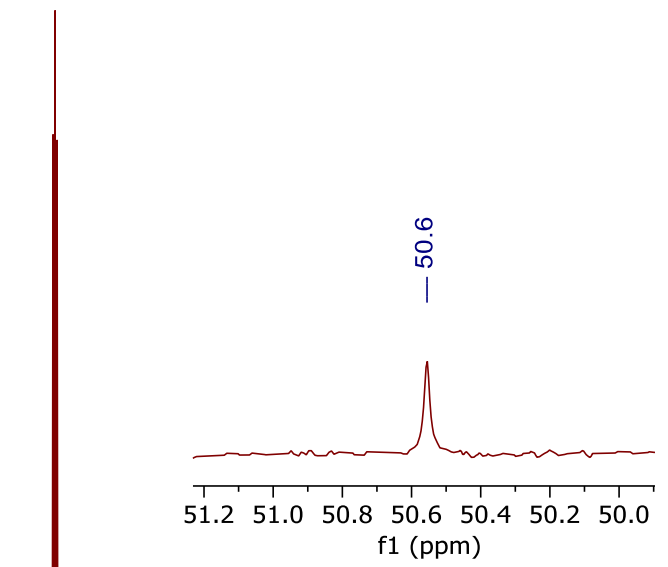

$$
\mathrm{f1} \text { (ppm) }
$$

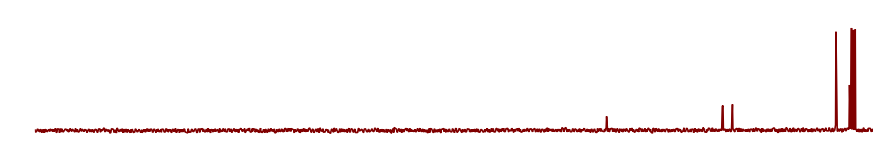


13. NMR Spectra of organometallic compounds 
* Tol-d

- Complex $\left[\left(\mathrm{L}_{1}\right)_{2} \mathrm{Ni}\right](7)$

complex $\left[\left(\mathrm{L}_{1}\right) \mathrm{Ni}(\operatorname{cod})\right](8)$

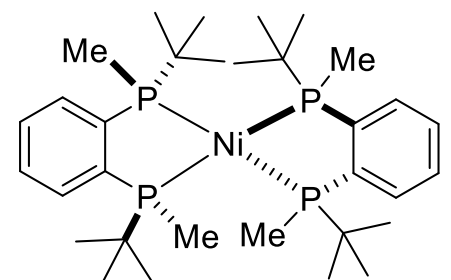

$\left[\left(\mathrm{L}_{1}\right)_{2} \mathrm{Ni}\right](\mathbf{7})$

${ }^{1} \mathrm{H}$ NMR (500 MHz, Toluene- $\left.d_{8}\right)$

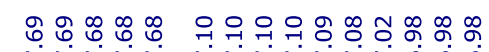

证
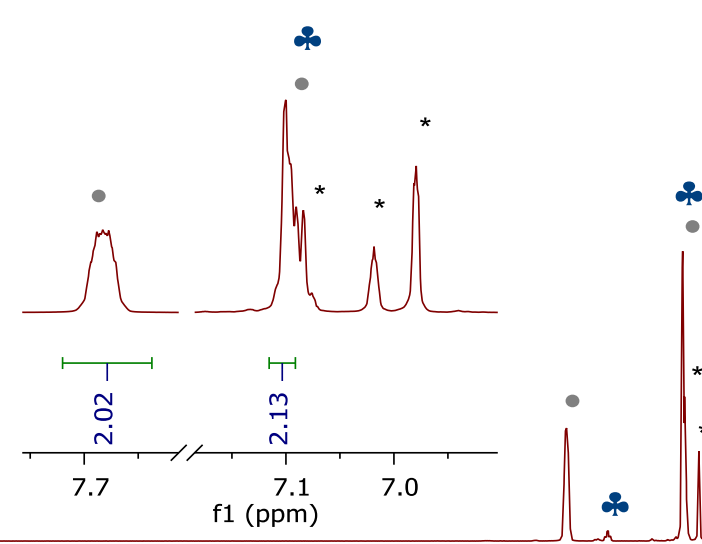

?.

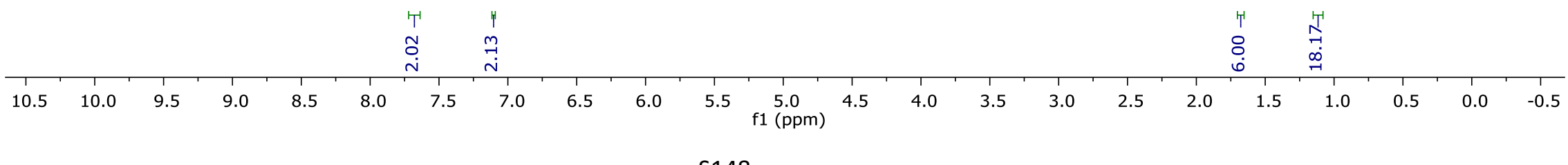




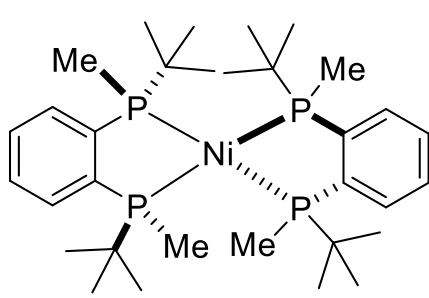

$\left[\left(\mathrm{L}_{1}\right)_{2} \mathrm{Ni}\right](7)$

${ }^{13} \mathrm{C}\left\{{ }^{1} \mathrm{H}\right\}$ NMR $\left(130 \mathrm{MHz}\right.$, Toluene- $\left.d_{8}\right)$

* Tol- $d_{n}$

- Complex $\left[\left(\mathrm{L}_{1}\right)_{2} \mathrm{Ni}\right](7)$

क. Complex $\left[\left(\mathrm{L}_{1}\right) \mathrm{Ni}(\operatorname{cod})\right](8)$

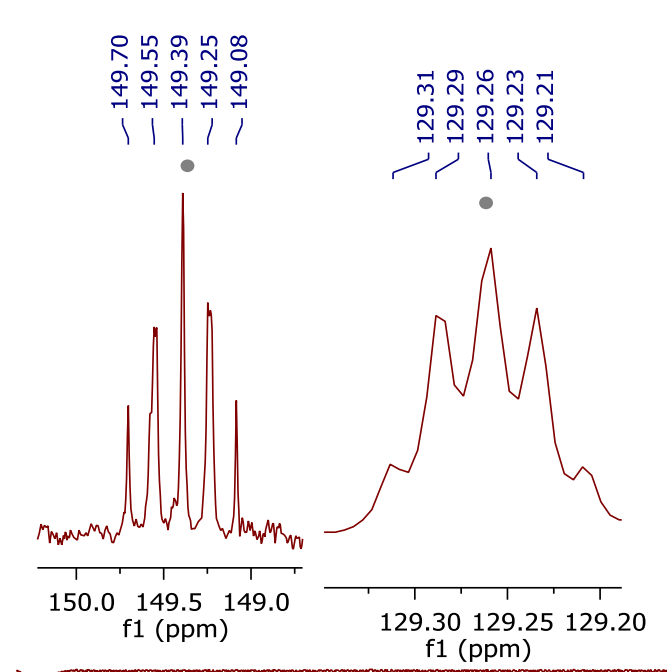

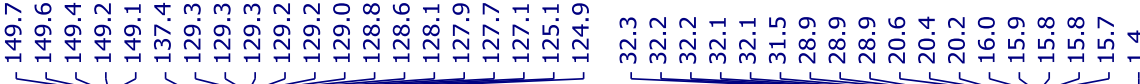

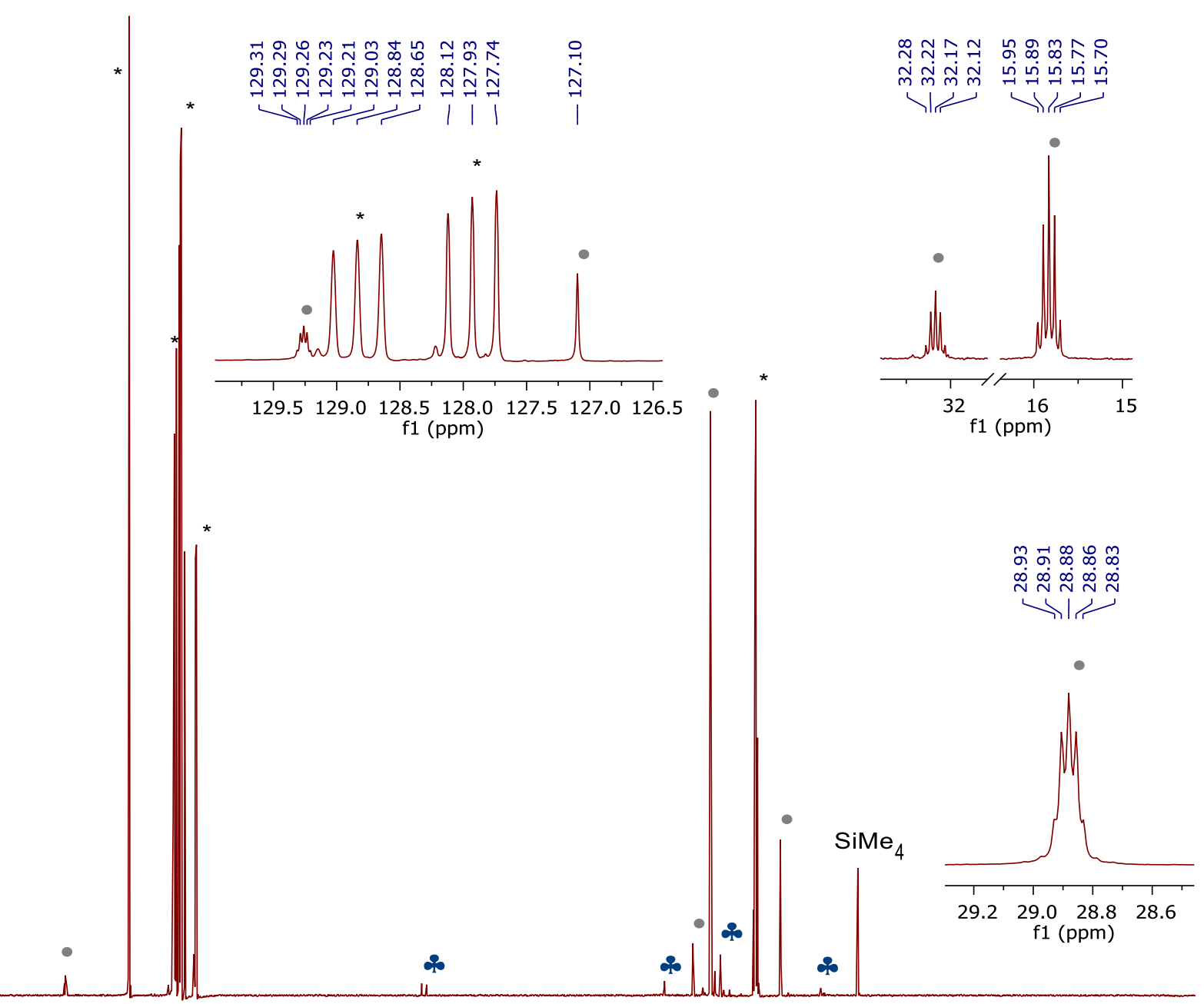

$\begin{array}{lllllllllllllllll}260 & 250 & 240 & 230 & 220 & 210 & 200 & 190 & 180 & 170 & 160 & 150 & 140 & 130 & 120 & 110 & 100\end{array}$ 


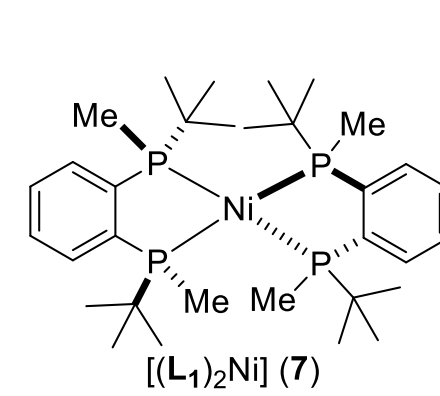

${ }^{31} \mathrm{P}$ NMR (160 MHz, Toluene- $d_{8}$ )

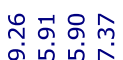

守 भ⿺辶े

r)

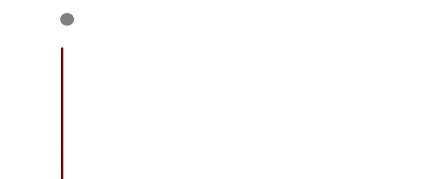

- Complex $\left[\left(\mathrm{L}_{1}\right)_{2} \mathrm{Ni}\right](7)$

- Complex $\left[\left(\mathrm{L}_{1}\right) \mathrm{Ni}(\mathrm{cod})\right](\mathbf{8})$

$\mathrm{L}_{1}$ monophosphine oxide

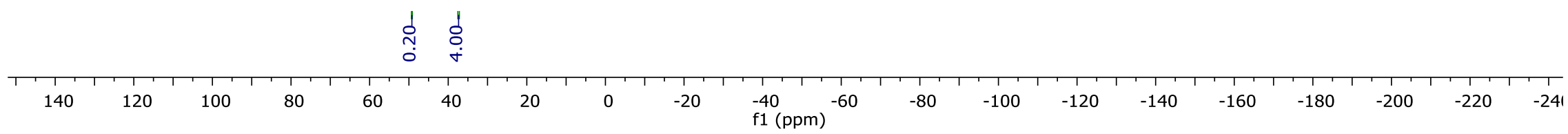




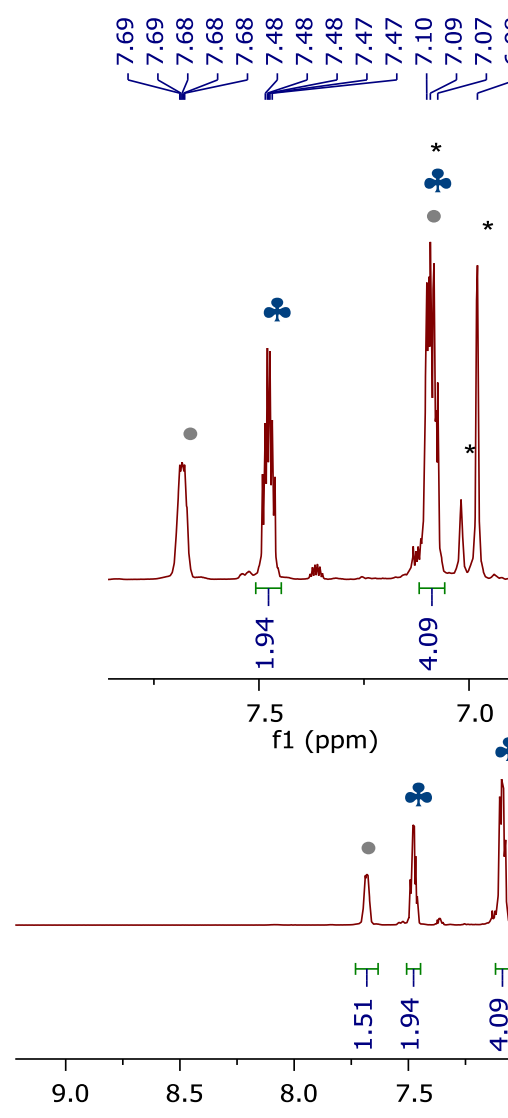

웅

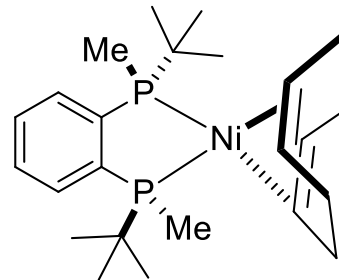

$\left[\left(\mathrm{L}_{1}\right) \mathrm{Ni}(\operatorname{cod})\right](8)$

${ }^{1} \mathrm{H}$ NMR $\left(500 \mathrm{MHz}\right.$, Toluene- $\left.d_{8}\right)$

* Tol- $d_{n}$

- Complex $\left[\left(\mathrm{L}_{1}\right)_{2} \mathrm{Ni}\right](7)$

\& Complex $\left[\left(\mathrm{L}_{1}\right) \mathrm{Ni}(\operatorname{cod})\right](8)$
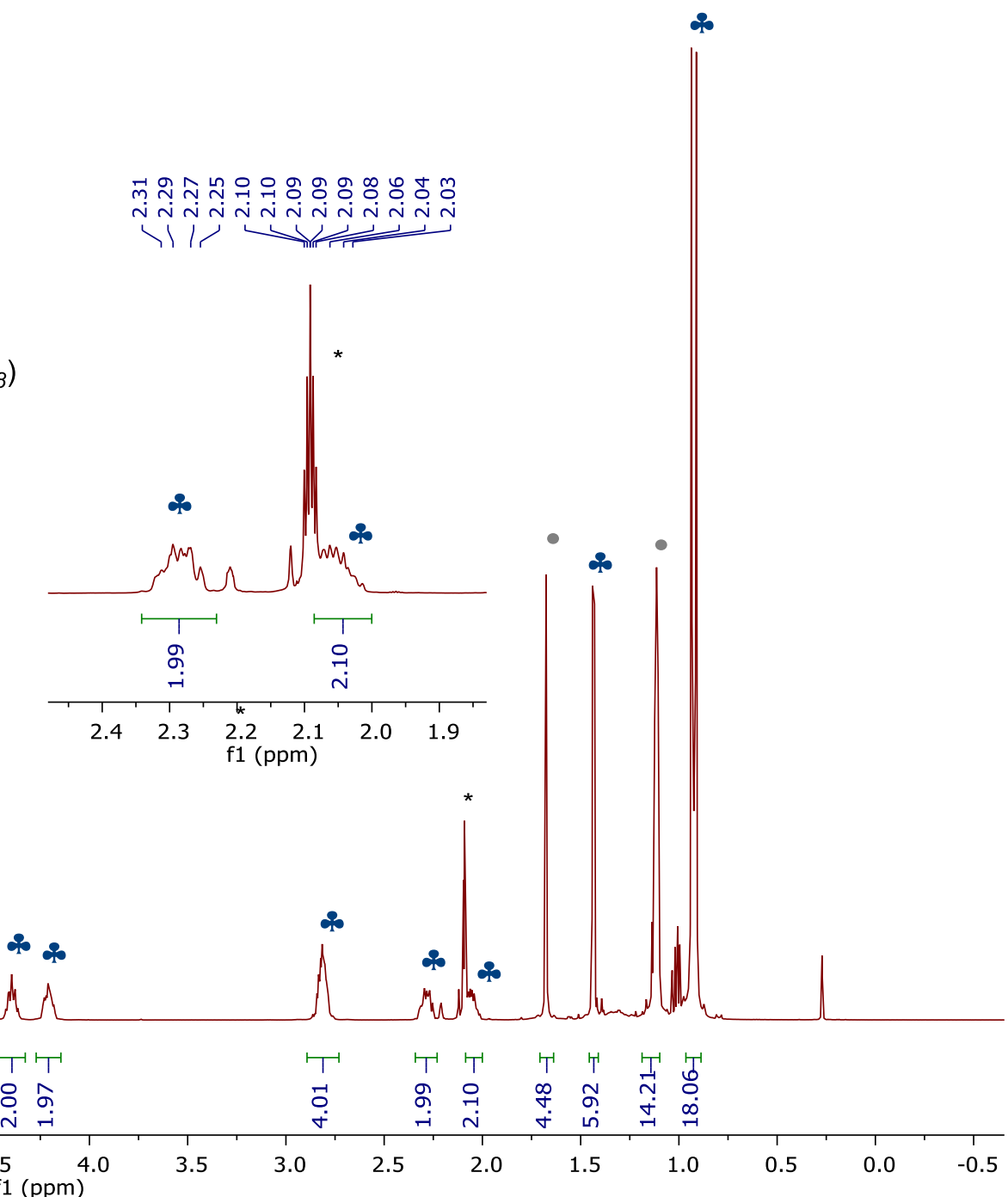


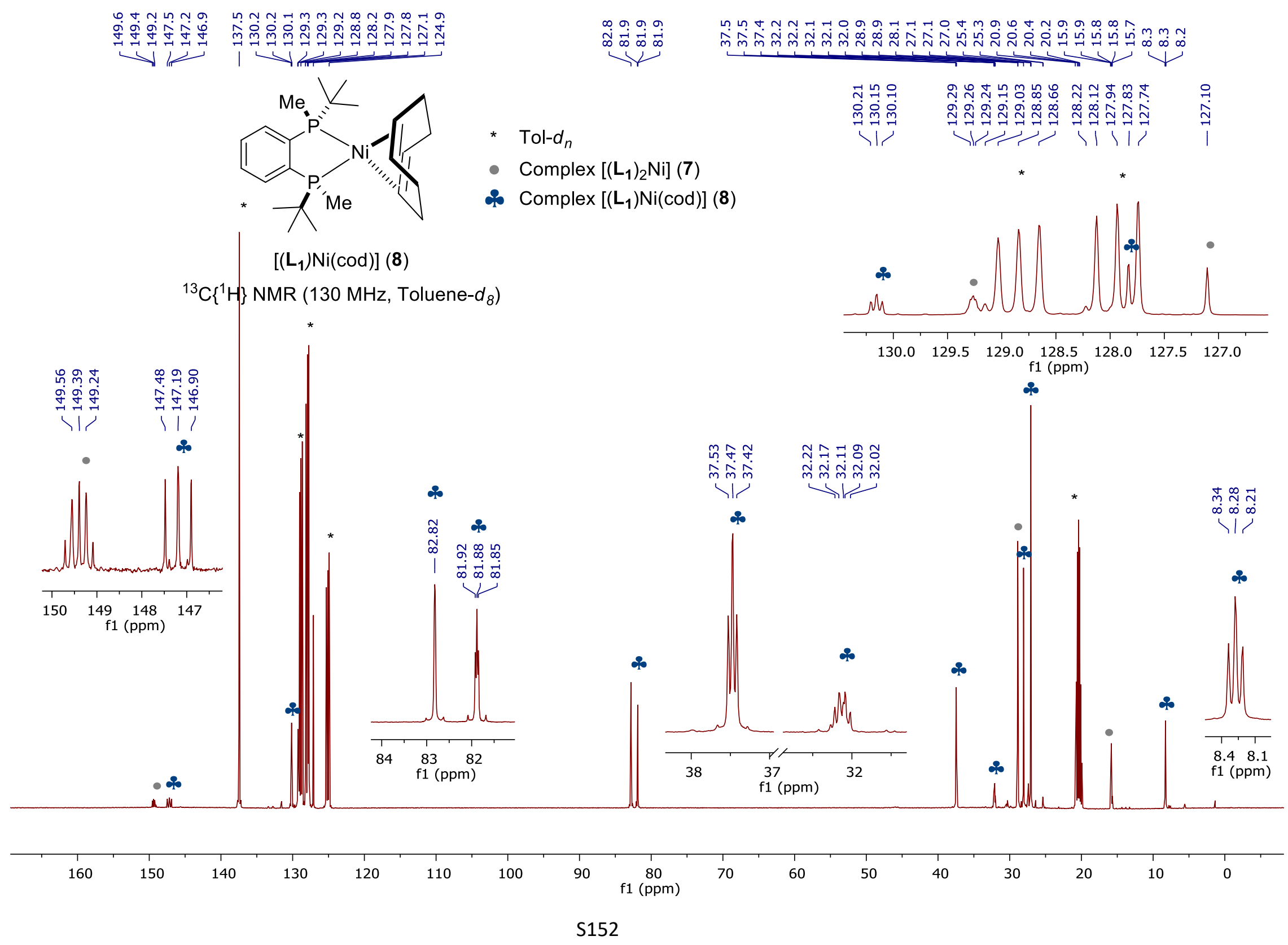




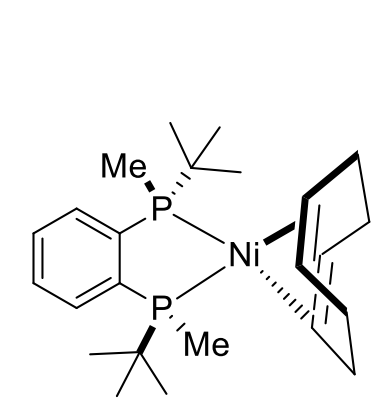

$\left[\left(\mathrm{L}_{1}\right) \mathrm{Ni}(\mathrm{cod})\right](\mathbf{8})$

${ }^{31} \mathrm{P}$ NMR (160 MHz, Toluene- $d_{8}$ ) $\begin{array}{cl}\overrightarrow{7} & 0 \\ 0 & 0 \\ 0 & 0 \\ i & i\end{array}$

- Complex $\left[\left(\mathrm{L}_{1}\right)_{2} \mathrm{Ni}\right](7)$

क. Complex $\left[\left(L_{1}\right) \mathrm{Ni}(\mathrm{cod})\right](8)$

- $\mathbf{L}_{1}$

$L_{1}$ monophosphine oxide

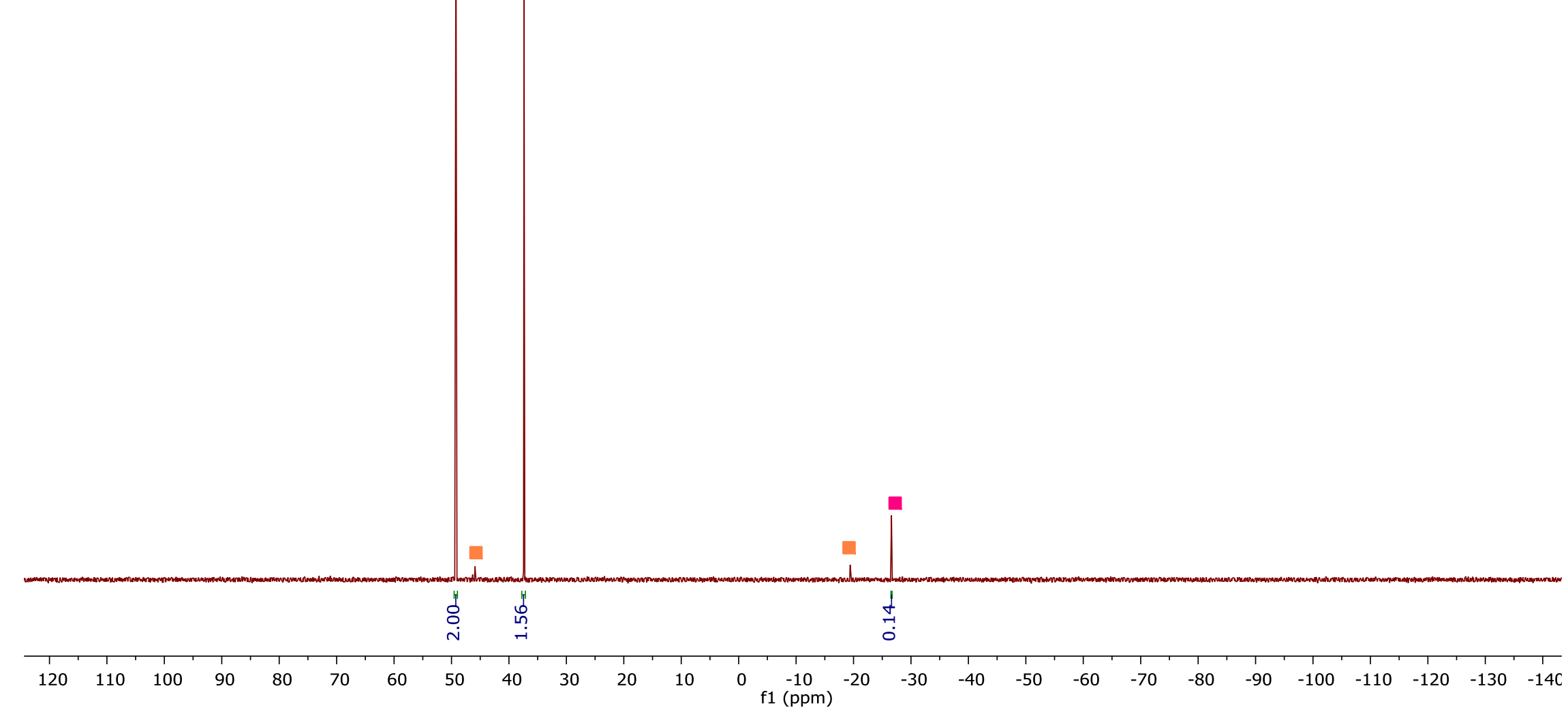




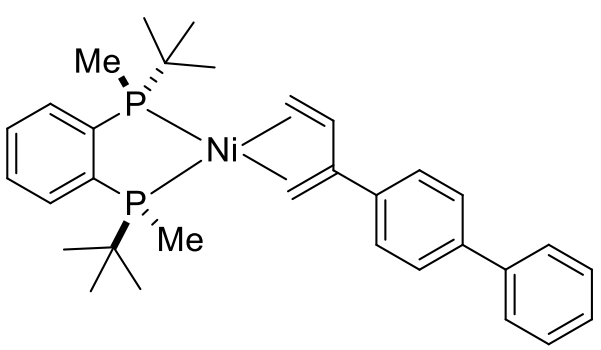

$\left[\left(\mathbf{L}_{1}\right) \mathrm{Ni}(\mathbf{1 a})\right](\mathbf{9})$

${ }^{1} \mathrm{H}$ NMR $\left(500 \mathrm{MHz}\right.$, Toluene- $\left.d_{8}\right)$

* Tol- $d_{n}$

a) Complex $\left[\left(\mathrm{L}_{1}\right) \mathrm{Ni}(\operatorname{cod})\right](8)$

$\checkmark$ Complex $\left[\left(\mathrm{L}_{1}\right) \mathrm{Ni}(1 \mathrm{a})\right](9)$

1a

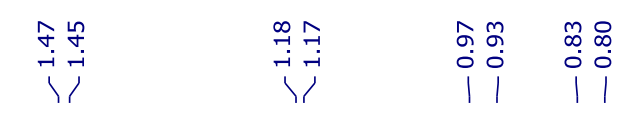
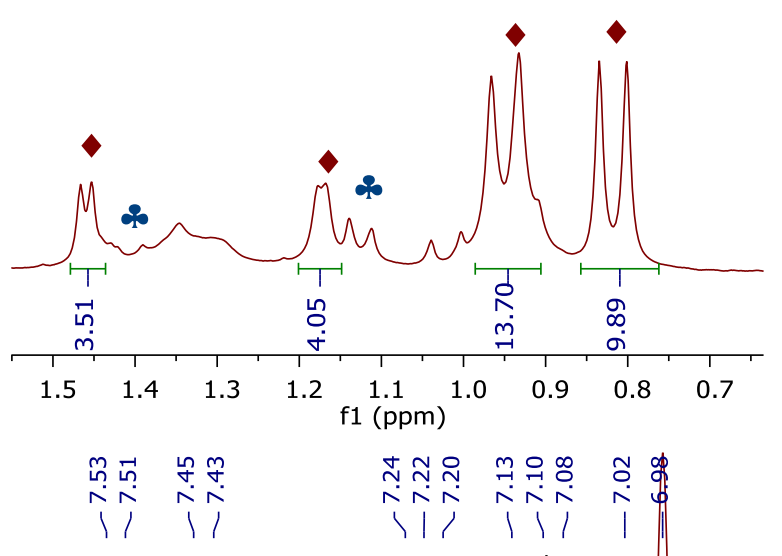

î i i
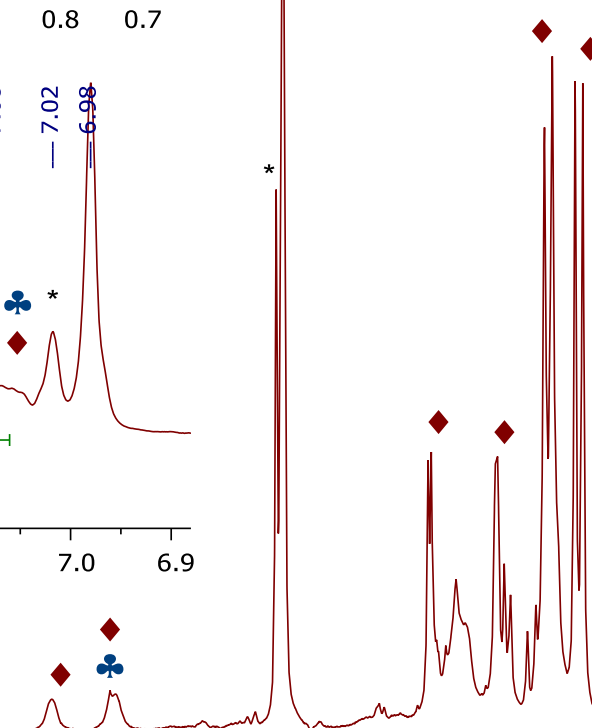

T $\begin{array}{ccc}T & T & T\end{array}$

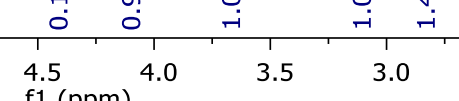

2.5 


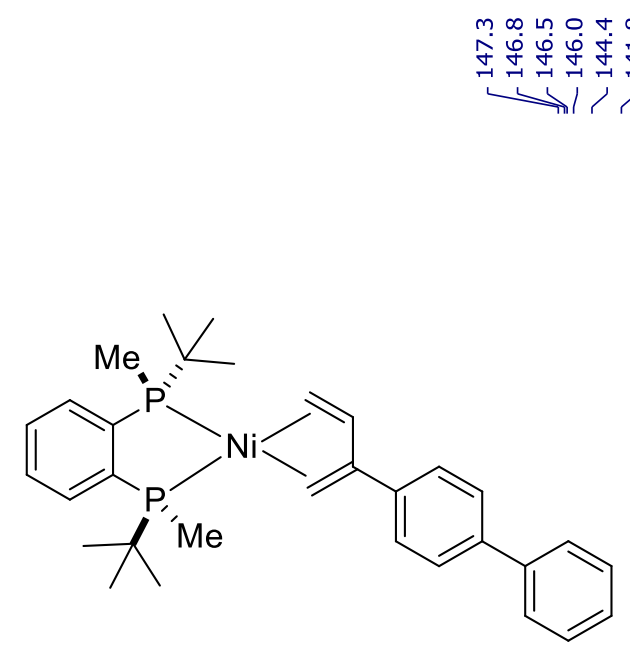

[( $\left.\left.\mathbf{L}_{1}\right) \mathrm{Ni}(\mathbf{1 a})\right](\mathbf{9})$

${ }^{13} \mathrm{C}\left\{{ }^{1} \mathrm{H}\right\}$ NMR $\left(130 \mathrm{MHz}\right.$, Toluene- $\left.d_{8}\right)$

* Tol- $d_{n}$

\&. Complex $\left[\left(\mathrm{L}_{1}\right) \mathrm{Ni}(\operatorname{cod})\right](8)$

$\checkmark$ Complex $\left[\left(\mathrm{L}_{1}\right) \mathrm{Ni}(\mathbf{1 a})\right](\mathbf{9})$

- 1a

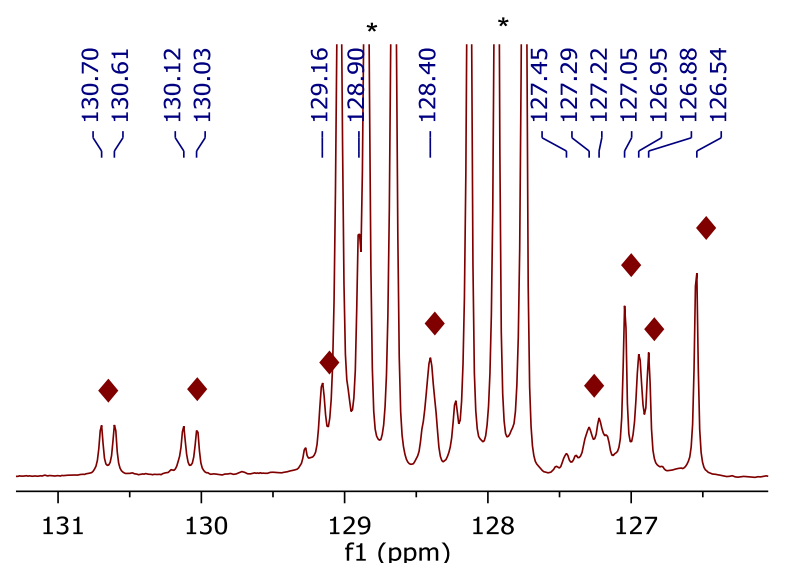

jum mos a t.m

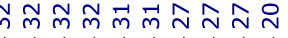
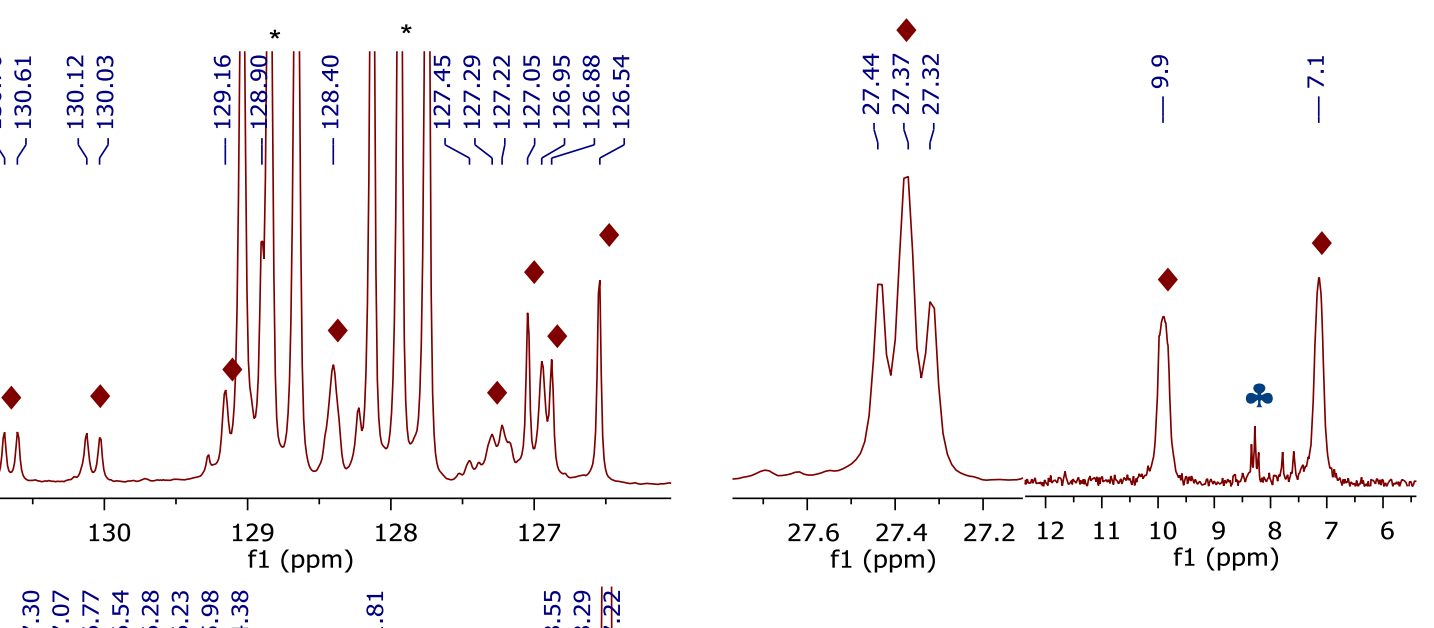

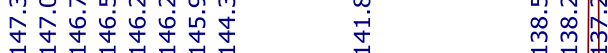

닌

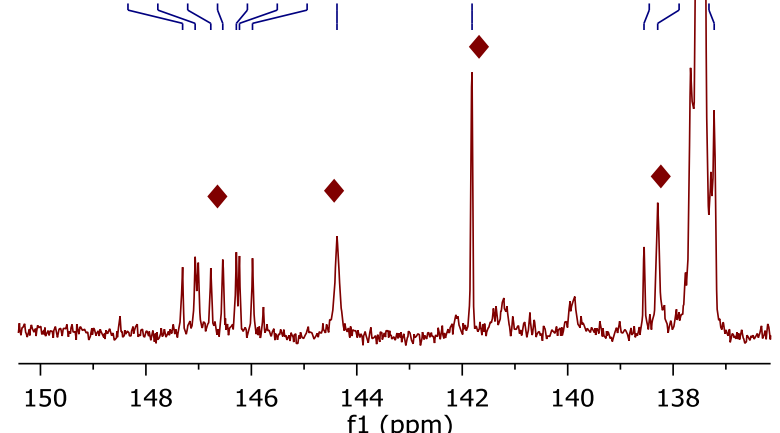

눈해
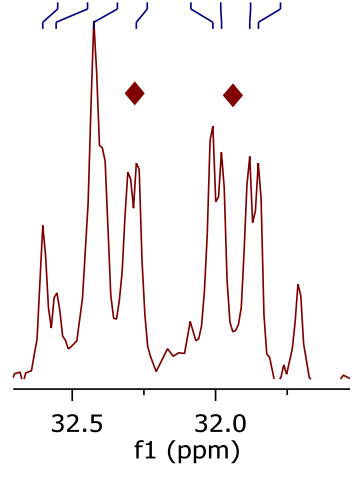
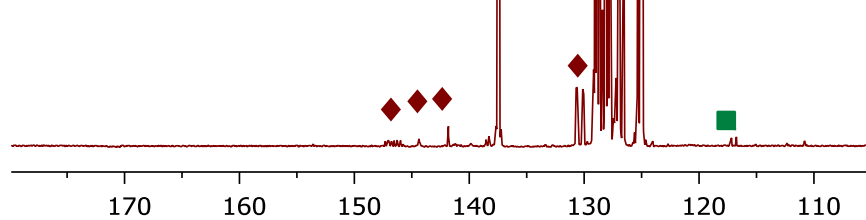

100

90 


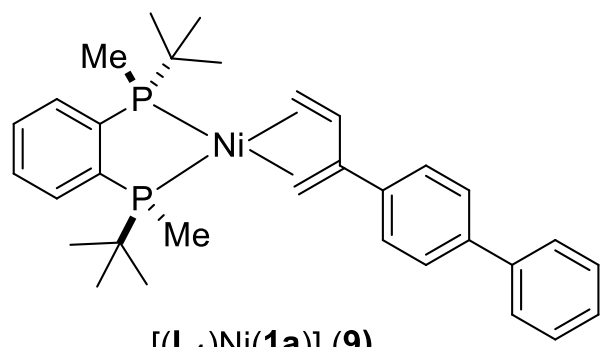

$\left[\left(\mathbf{L}_{1}\right) \mathrm{Ni}(\mathbf{1 a})\right](\mathbf{9})$

${ }^{31} \mathrm{P}$ NMR $\left(160 \mathrm{MHz}\right.$, Toluene- $d_{8}$ )

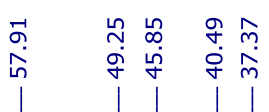

Complex $\left[\left(\mathrm{L}_{1}\right)_{2} \mathrm{Ni}\right](\mathbf{7})$

a Complex $\left[\left(\mathrm{L}_{1}\right) \mathrm{Ni}(\operatorname{cod})\right](8)$

$\checkmark$ Complex $\left[\left(\mathrm{L}_{\mathbf{1}}\right) \mathrm{Ni}(\mathbf{1} \mathbf{a})\right](\mathbf{9})$

- $\mathrm{L}_{1}$ monophosphine oxide

m̊n

पे

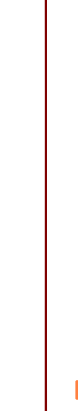
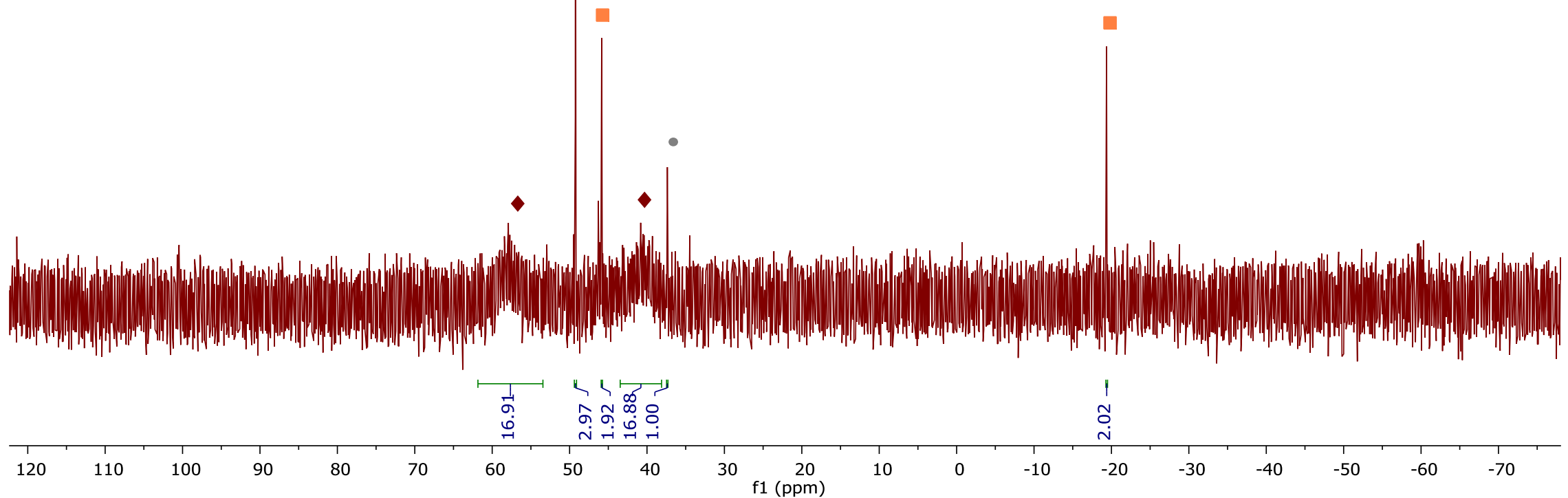
$\left[\left(\mathrm{L}_{1}\right) \mathrm{Ni}(\mathbf{1 a})\right](\mathbf{9})$

${ }^{31} \mathrm{P}$ NMR $\left(120 \mathrm{MHz}\right.$, Toluene- $\left.d_{8}\right)$

Variable temperature (168 to $298 \mathrm{~K}$ )

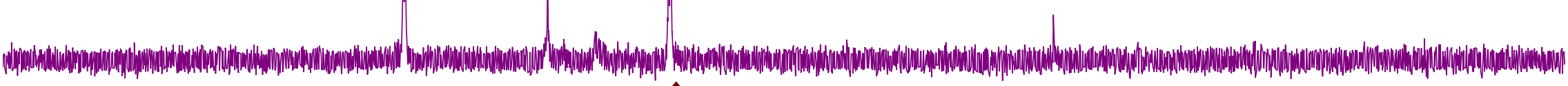

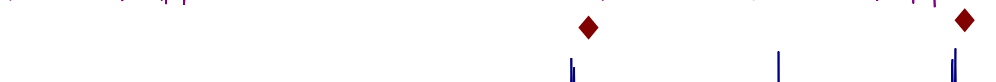

|

$\diamond$ i

\section{1}

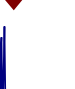

a) Complex $\left[\left(\mathrm{L}_{1}\right) \mathrm{Ni}(\operatorname{cod})\right](8)$

$\checkmark$ Complex $\left[\left(\mathrm{L}_{\mathbf{1}}\right) \mathrm{Ni}(\mathbf{1 a})\right](\mathbf{9})$

- $\mathrm{L}_{1}$ monophosphine oxide
$\mathrm{T}=168 \mathrm{~K}$

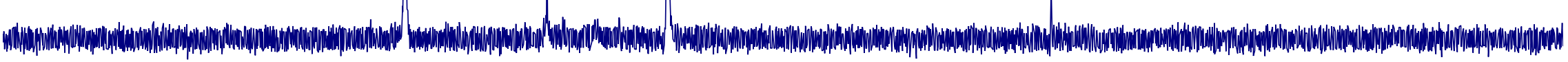
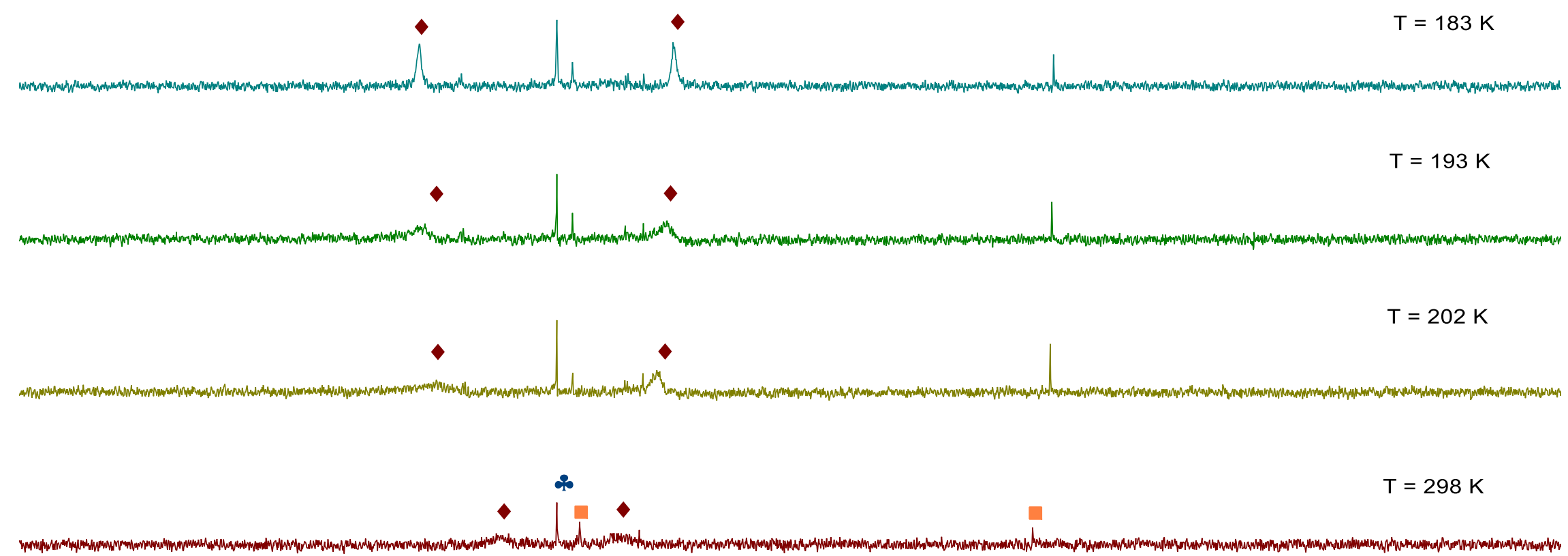


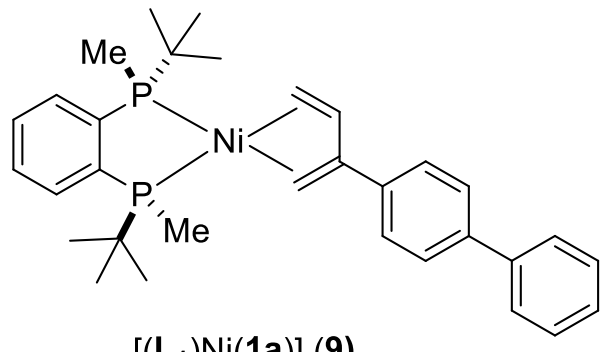

$\left[\left(\mathbf{L}_{\mathbf{1}}\right) \mathrm{Ni}(\mathbf{1 a})\right](\mathbf{9})$

${ }^{31} \mathrm{P}$ NMR $\left(120 \mathrm{MHz}\right.$, Toluene- $\left.d_{8}\right)$ $\mathrm{T}=168 \mathrm{~K}$
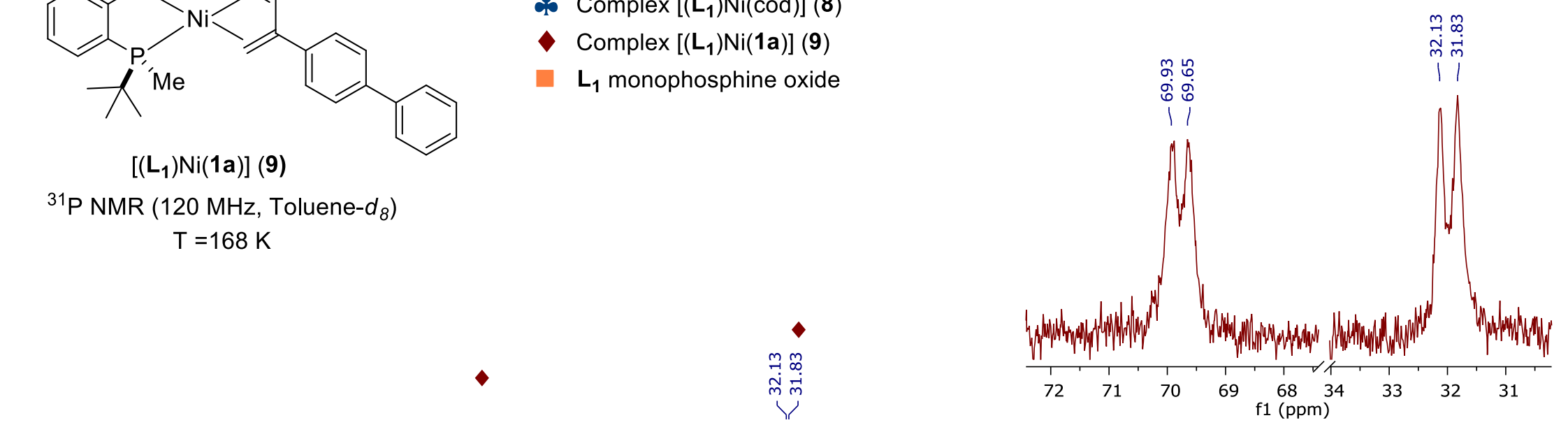

\& Complex $\left[\left(\mathrm{L}_{1}\right) \mathrm{Ni}(\mathrm{cod})\right](8)$

Complex $\left[\left(\mathrm{L}_{1}\right) \mathrm{Ni}(\mathbf{1 a})\right](\mathbf{9})$

- $\mathrm{L}_{1}$ monophosphine oxide

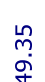

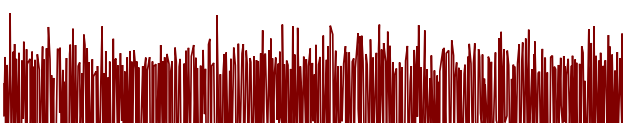

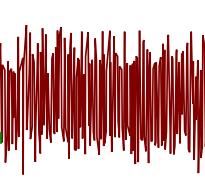

-

$110 \quad 100$

90

80

60

50

30
f1 $(\mathrm{ppm})$ 


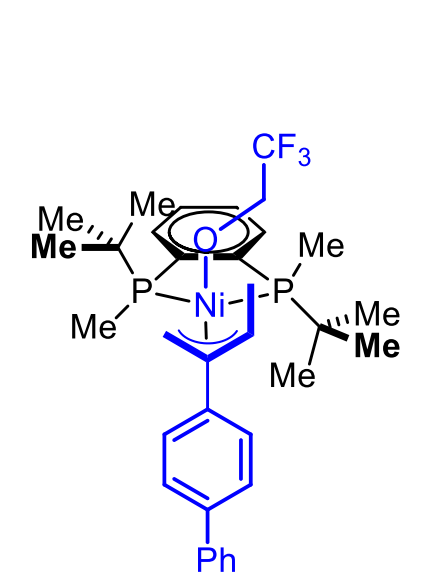

* Tol- $d_{n}$

- Complex $\left[\left(\mathrm{L}_{1}\right)_{2} \mathrm{Ni}\right](7)$

a. Complex $\left[\left(\mathrm{L}_{1}\right) \mathrm{Ni}(\mathrm{cod})\right](8)$

A. Complex $\left[\left(\mathrm{L}_{1}\right) \mathrm{Ni}\left(\eta^{3}\right.\right.$-allyl $\left.)\right]\left[\mathrm{OCH}_{2} \mathrm{CF}_{3}\right](\mathbf{1 0})$

- $\mathrm{CF}_{3} \mathrm{CH}_{2} \mathrm{OH}$

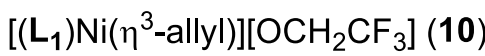

${ }^{1} \mathrm{H}$ NMR $\left(500 \mathrm{MHz}\right.$, Toluene- $\left.d_{8}\right)$

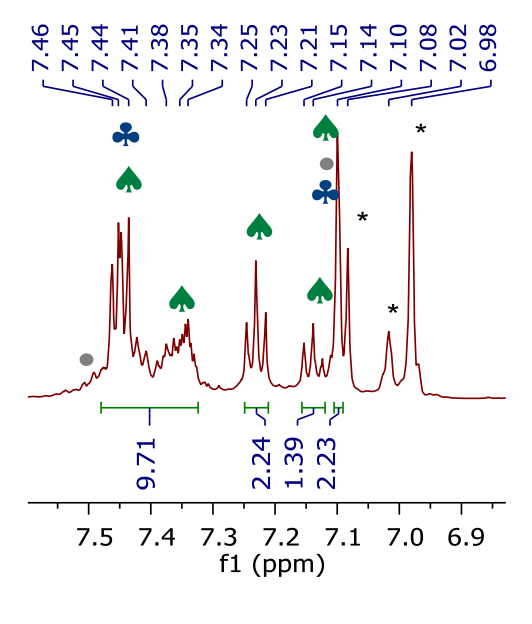

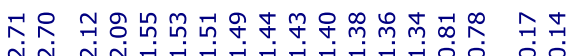

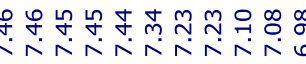

r

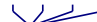

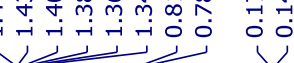

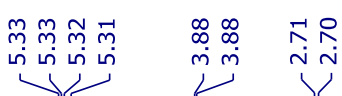

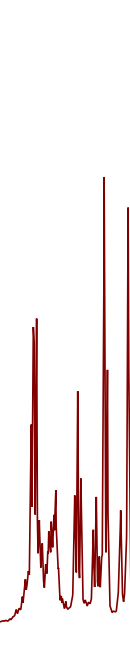

(
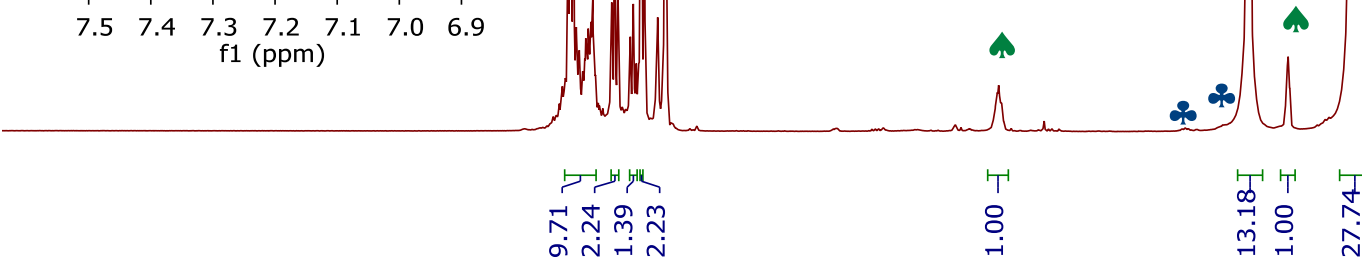

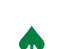

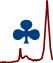
l.

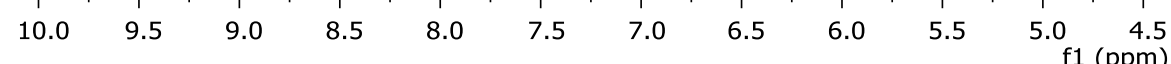




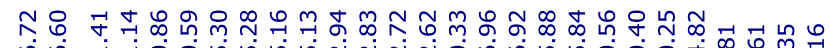

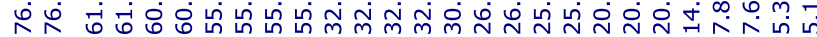

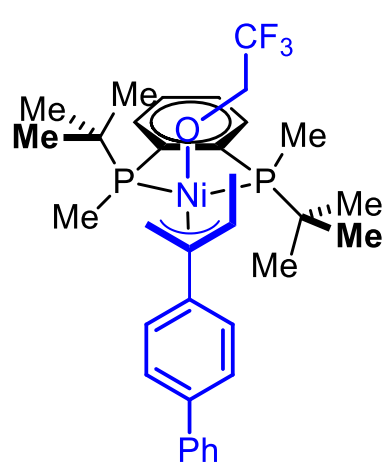

$\left[\left(\mathrm{L}_{1}\right) \mathrm{Ni}\left(\eta^{3}\right.\right.$-allyl)][OOCH $\left.\mathrm{CF}_{3}\right](\mathbf{1 0})$

${ }^{13} \mathrm{C}\left\{{ }^{1} \mathrm{H}\right\}$ NMR $\left(130 \mathrm{MHz}\right.$, Toluene- $\left.d_{8}\right)$

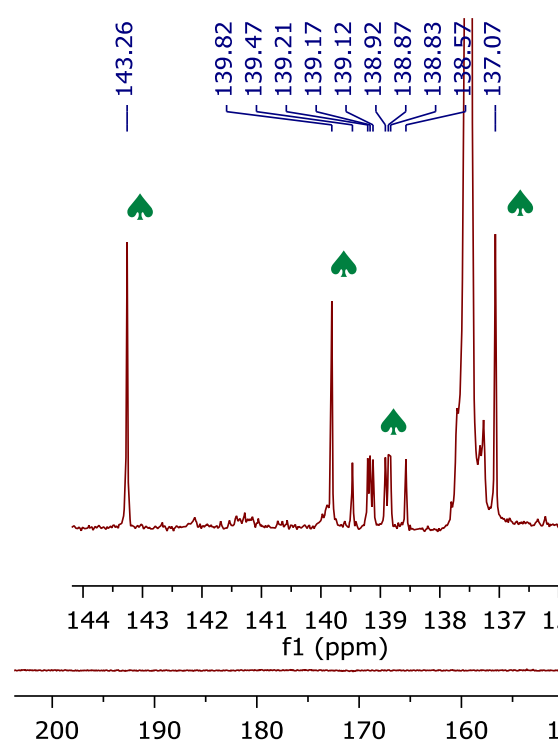

$\begin{array}{lllll}190 & 180 & 170 & 160 & 150\end{array}$ $\longrightarrow$ U
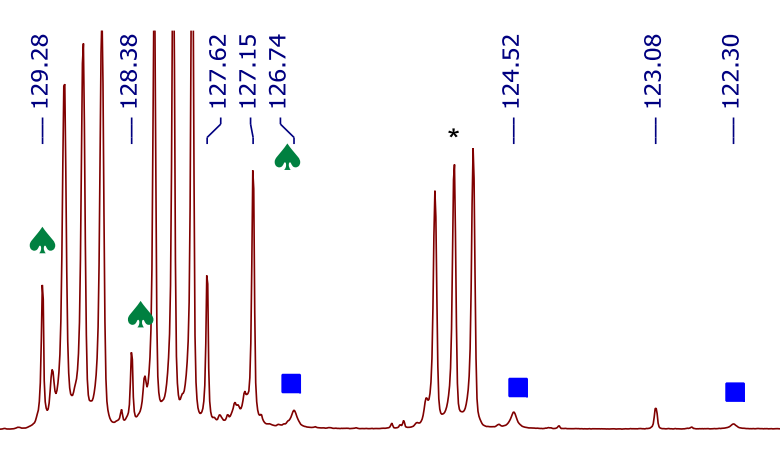

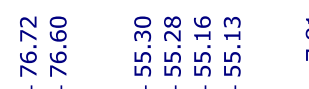

$\stackrel{\infty}{i}$

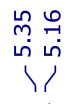

a
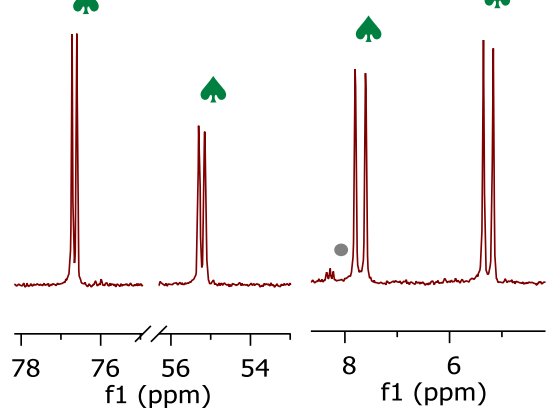

कू⿻ $\prod_{1}^{m} m$

* Tol- $d_{n}$

- Complex $\left[\left(\mathrm{L}_{1}\right)_{2} \mathrm{Ni}\right](7)$

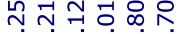

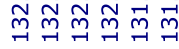

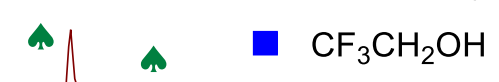

Complex $\left[\left(\mathrm{L}_{1}\right) \mathrm{Ni}(\mathrm{cod})\right](8)$

4. Complex $\left[\left(\mathrm{L}_{1}\right) \mathrm{Ni}\left(\eta^{3}\right.\right.$-allyl $\left.)\right]\left[\mathrm{OCH}_{2} \mathrm{CF}_{3}\right](10)$
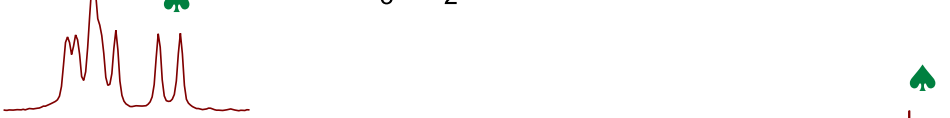

$132.5 \quad 132.0 \quad 131.5$ f1 (ppm)

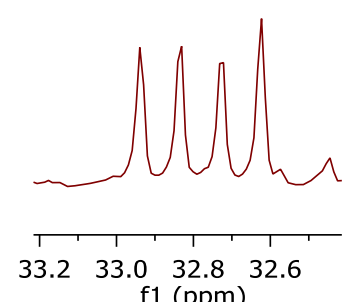

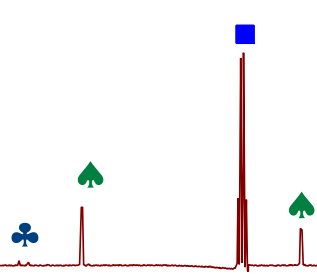




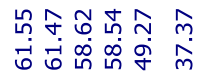

نुน

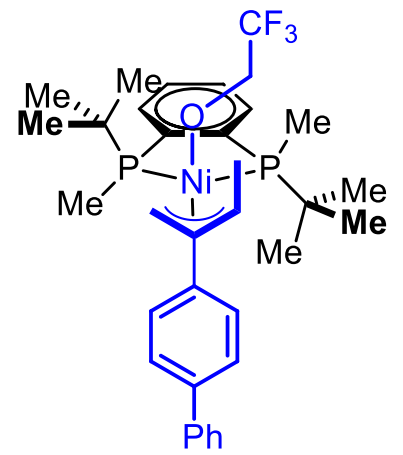

A $\quad\left[\left(\mathrm{L}_{1}\right) \mathrm{Ni}\left(\eta^{3}\right.\right.$-allyl $\left.)\right]\left[\mathrm{OCH}_{2} \mathrm{CF}_{3}\right](10)$

${ }^{31} \mathrm{P}$ NMR $\left(160 \mathrm{MHz}\right.$, Toluene- $\left.d_{8}\right)$

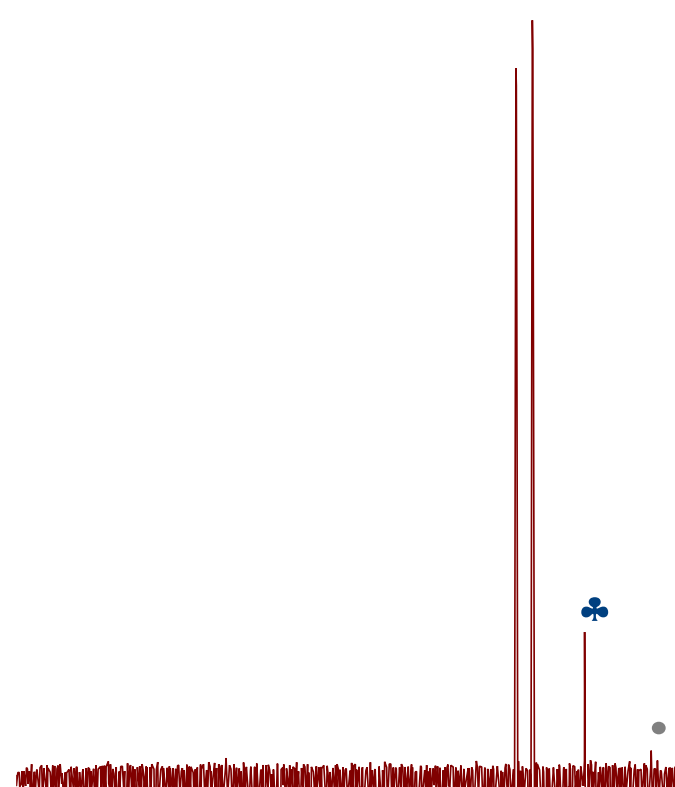

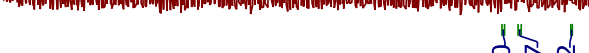

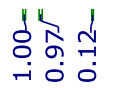

- Complex $\left[\left(\mathrm{L}_{1}\right)_{2} \mathrm{Ni}\right](7)$

is Complex $\left[\left(\mathrm{L}_{1}\right) \mathrm{Ni}(\operatorname{cod})\right](8)$

A. Complex $\left[\left(\mathrm{L}_{1}\right) \mathrm{Ni}\left(\eta^{3}\right.\right.$-allyl) $]\left[\mathrm{OCH}_{2} \mathrm{CF}_{3}\right](\mathbf{1 0})$

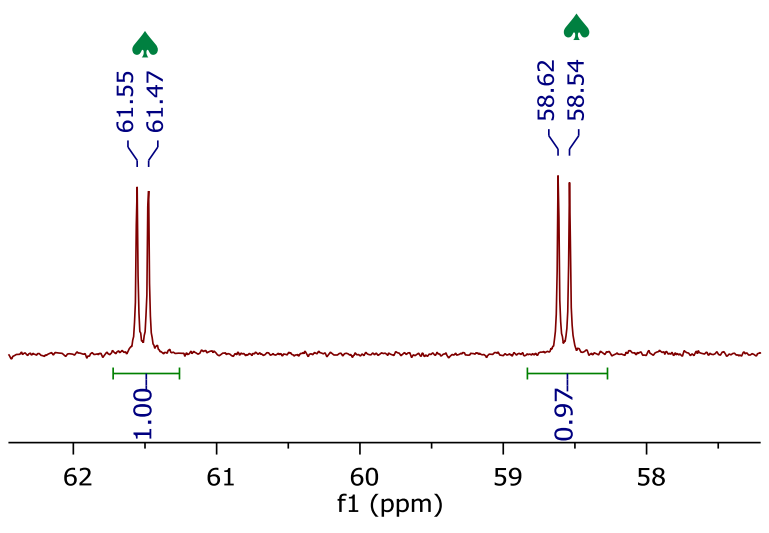

50

110

90

$70 \quad 50$

30

10

$\stackrel{-50}{f 1}$ 


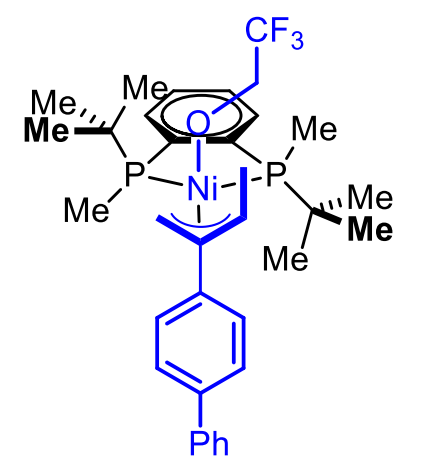

- $\mathrm{CF}_{3} \mathrm{CH}_{2} \mathrm{OH}$

$\left[\left(\mathrm{L}_{1}\right) \mathrm{Ni}\left(\eta^{3}\right.\right.$-ally) $)\left[\mathrm{OCH}_{2} \mathrm{CF}_{3}\right](\mathbf{1 0})$

${ }^{19} \mathrm{~F}\left\{{ }^{1} \mathrm{H}\right\}$ NMR $\left(280 \mathrm{MHz}\right.$, Toluene- $\left.d_{8}\right)$

$$
\substack { \infty \\
\begin{subarray}{c}{i \\
i{ \infty \\
\begin{subarray} { c } { i \\
i } } \end{subarray}
$$

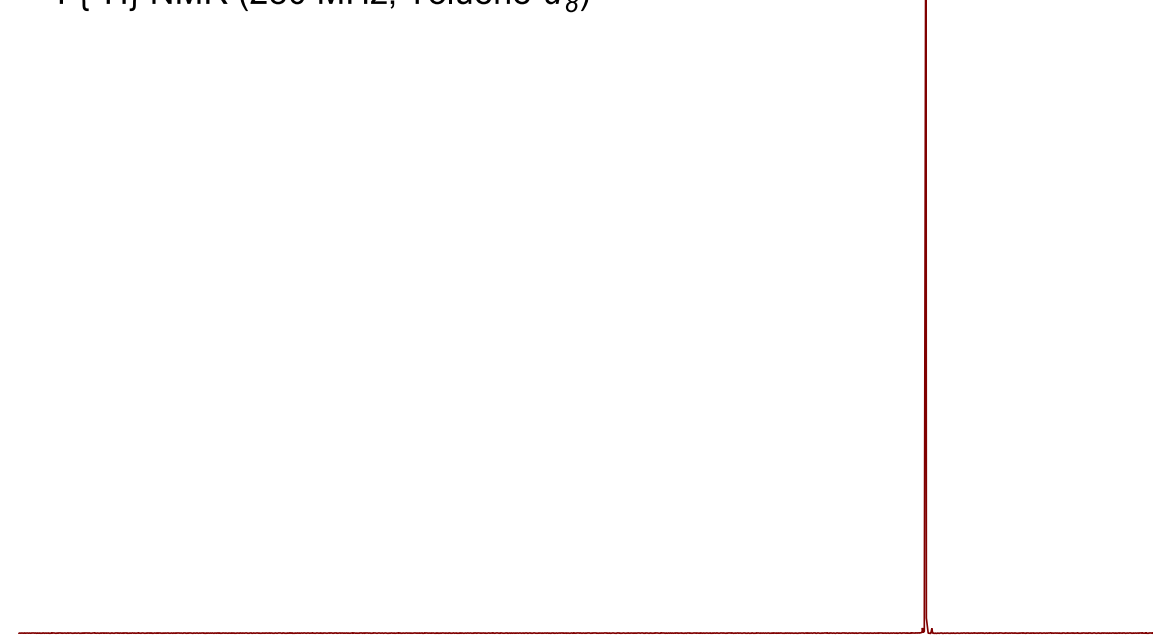

100 


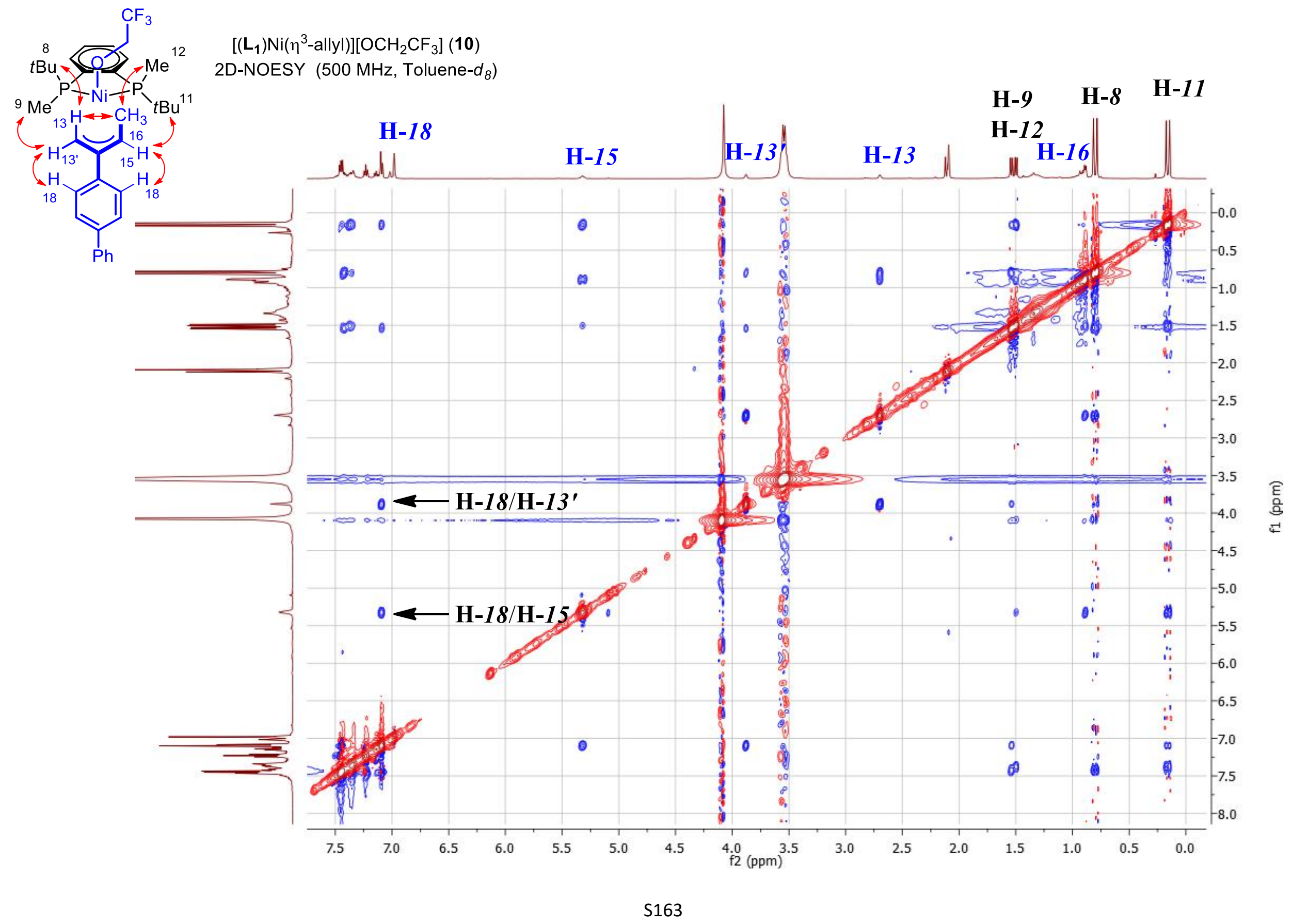




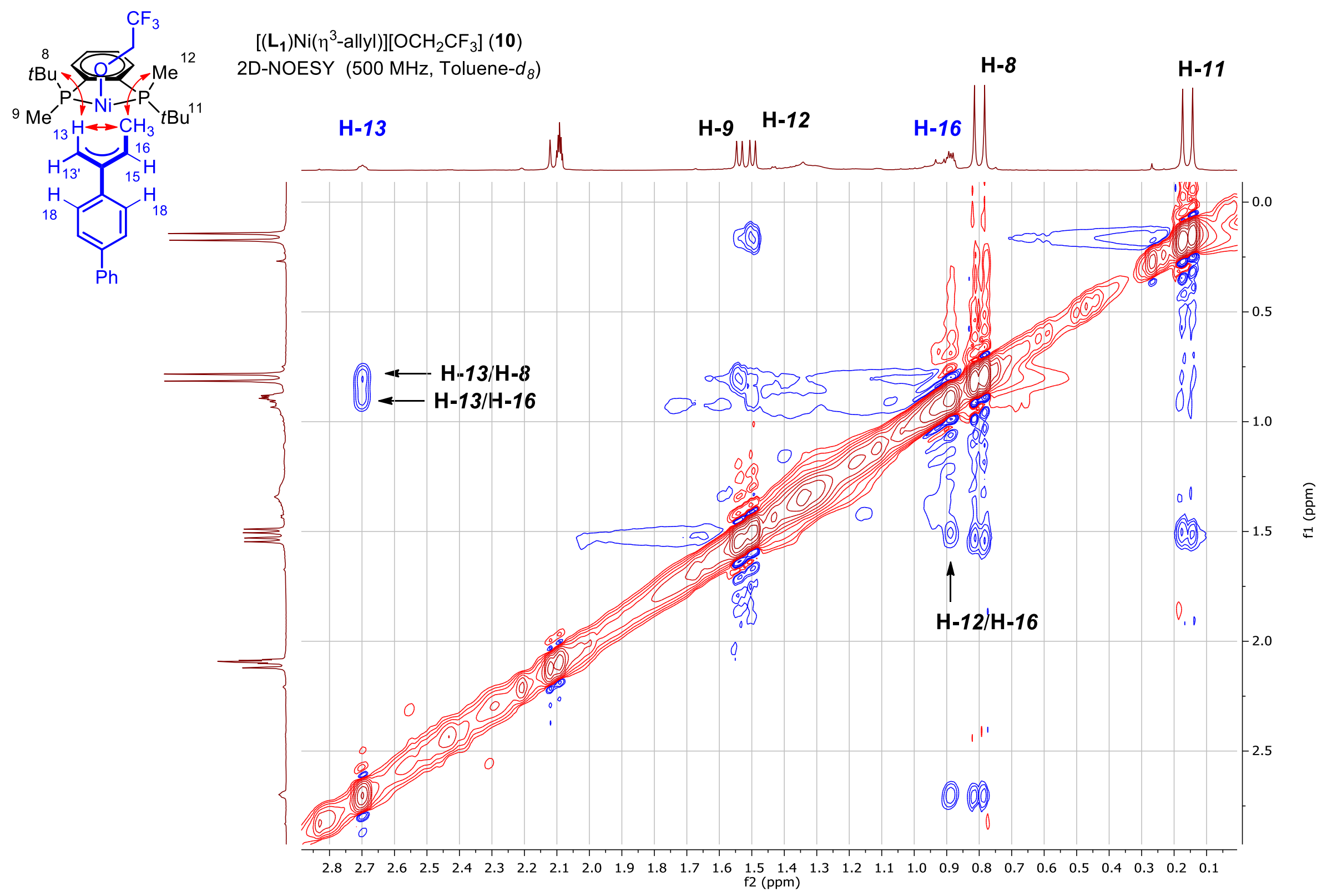




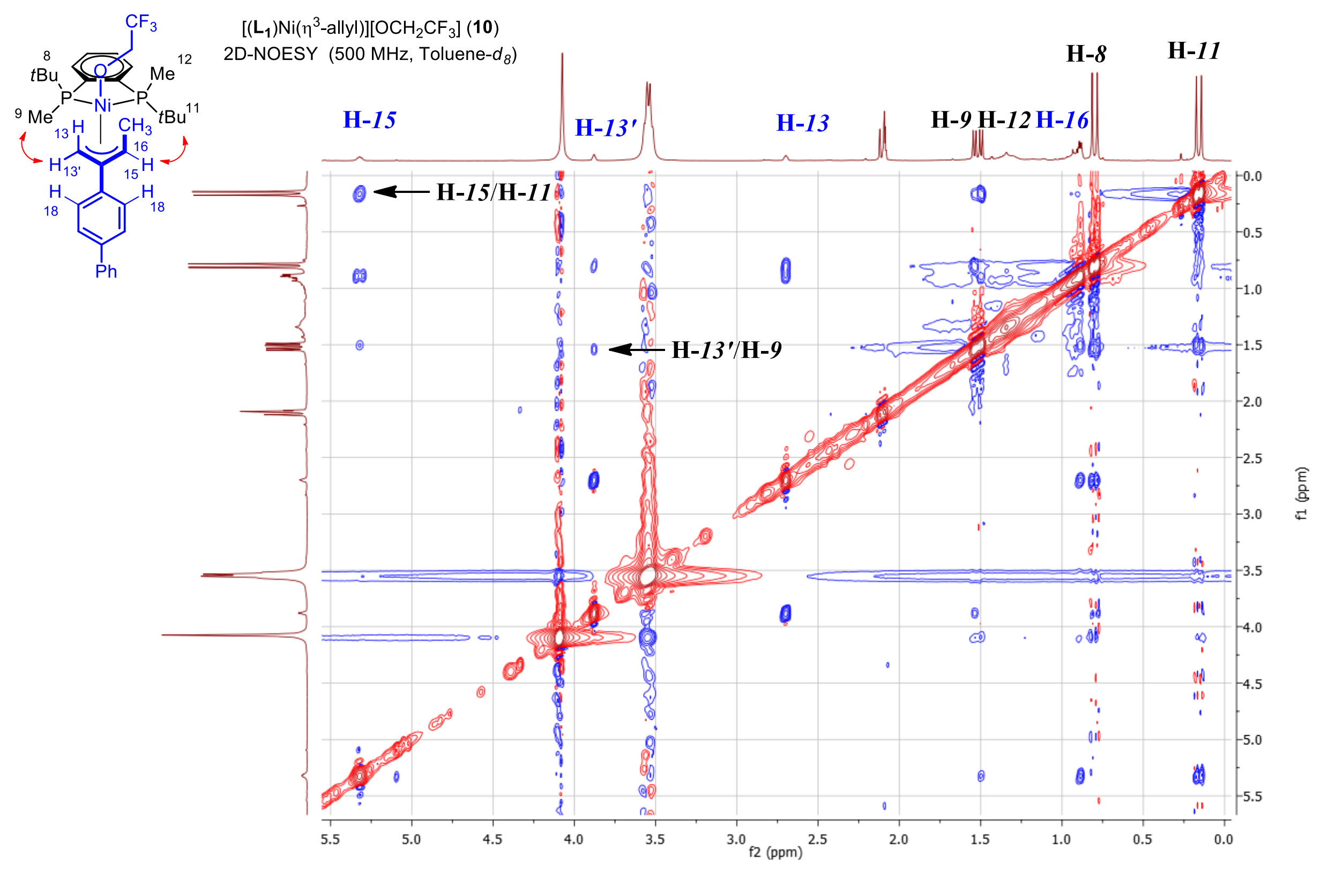




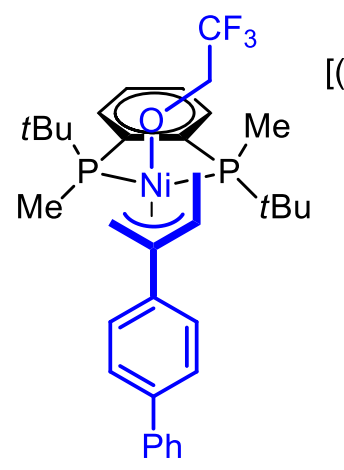

$\left[\left(\mathrm{L}_{1}\right) \mathrm{Ni}\left(\eta^{3}\right.\right.$-allyl)][ $\left[\mathrm{OCH}_{2} \mathrm{CF}_{3}\right](\mathbf{1 0})$

${ }^{19} \mathrm{~F},{ }^{1} \mathrm{H}$-HOESY (Toluene- $d_{8}$ )
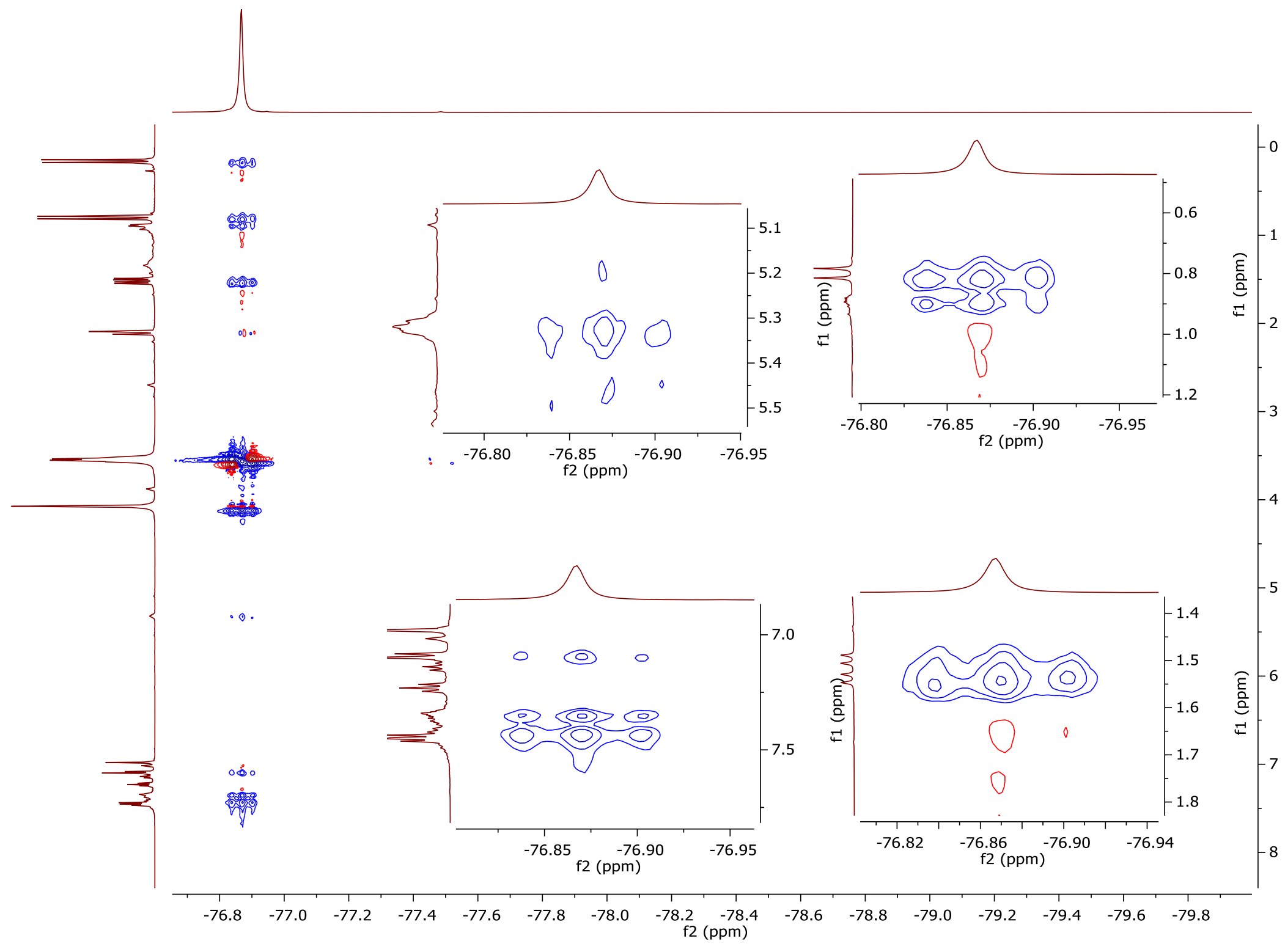

S166 


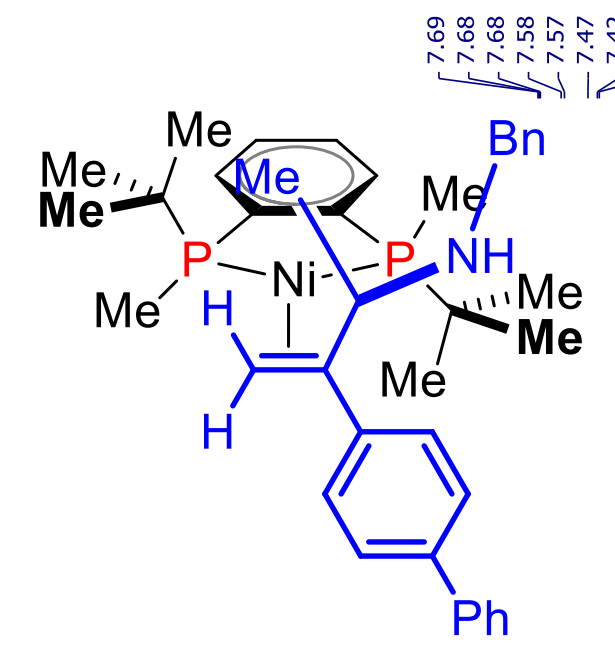

$\left[\left(\mathrm{L}_{1}\right) \mathrm{Ni}(3 \mathrm{aa})\right]$ (11-major)

${ }^{1} \mathrm{H}$ NMR (500 MHz, Toluene- $d_{8}$ )
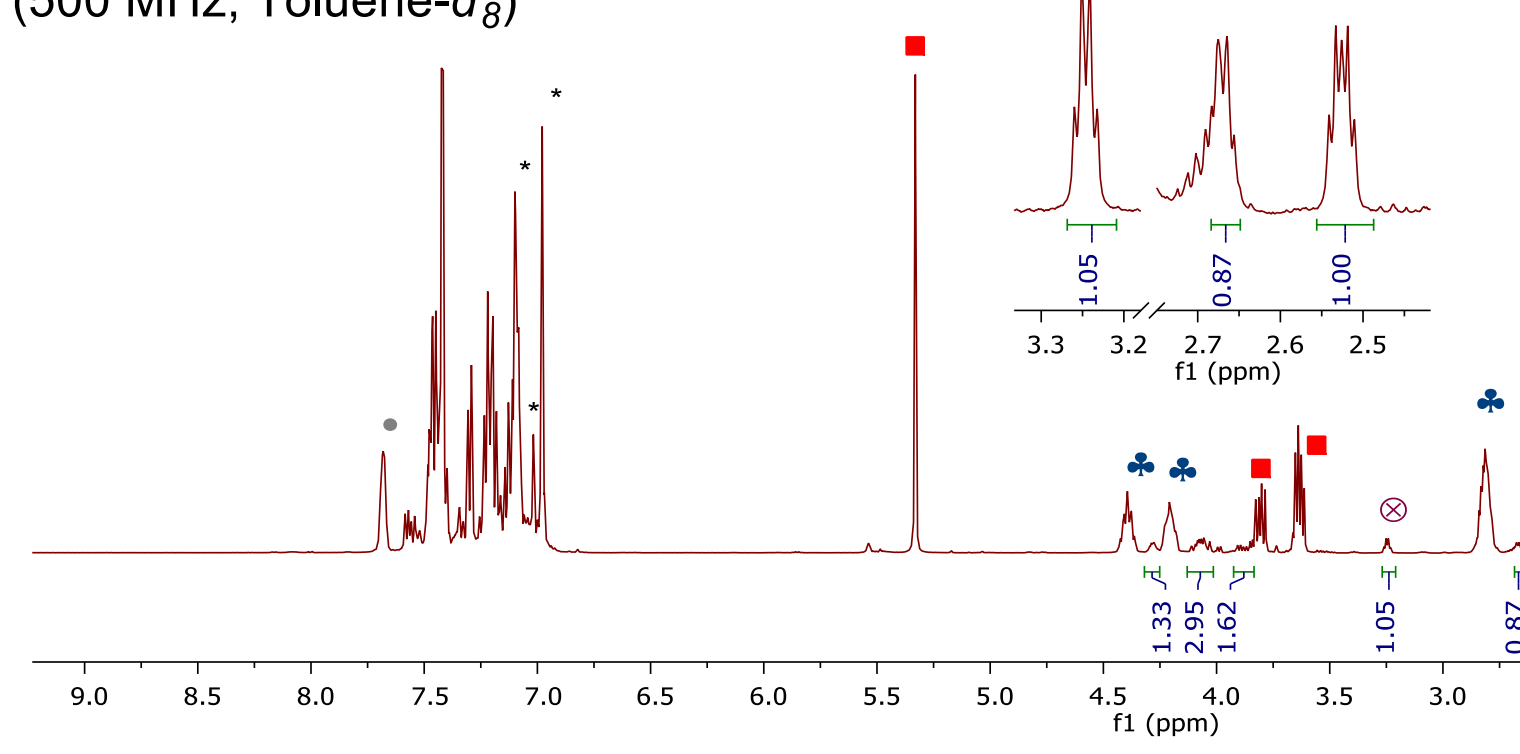

Tol- $d_{n}$

- Complex $\left[\left(\mathrm{L}_{1}\right)_{2} \mathrm{Ni}\right](7)$

a Complex $\left[\left(L_{1}\right) \mathrm{Ni}(\operatorname{cod})\right](8)$

$\otimes$ Complex $\left[\left(\mathbf{L}_{1}\right) \mathrm{Ni}(\mathbf{3 a a})\right]$ (11-major)

$\otimes$ Complex $\left[\left(L_{1}\right) \mathrm{Ni}(3 a a)\right]$ (11-minor)

3aa

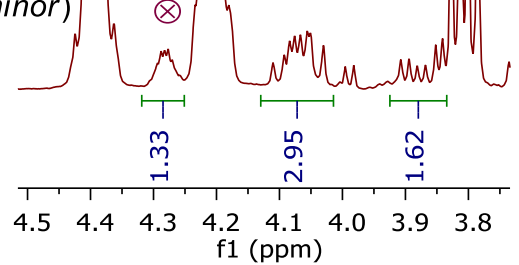

눙

$\underbrace{1}$

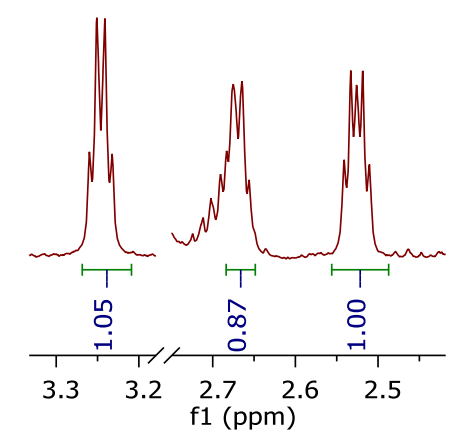

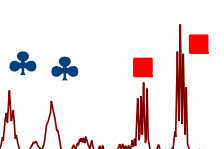

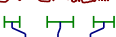




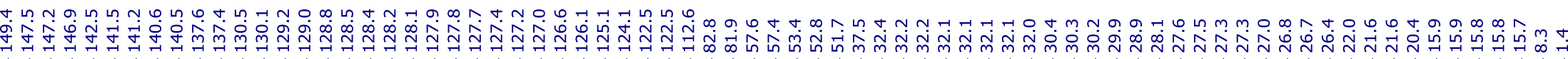

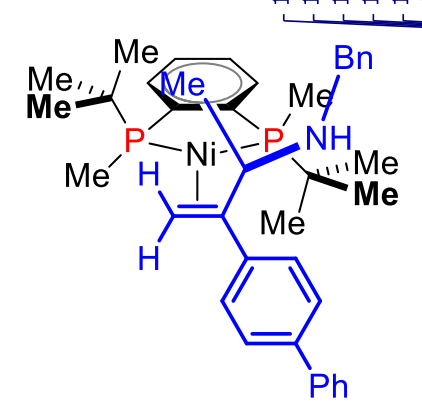

[( $\left.\left.\mathrm{L}_{1}\right) \mathrm{Ni}(\mathbf{3 a a})\right]$ (11-major)

${ }^{13} \mathrm{C}\left\{{ }^{1} \mathrm{H}\right\}$ NMR $\left(130 \mathrm{MHz}\right.$, Toluene- $\left.d_{8}\right)$

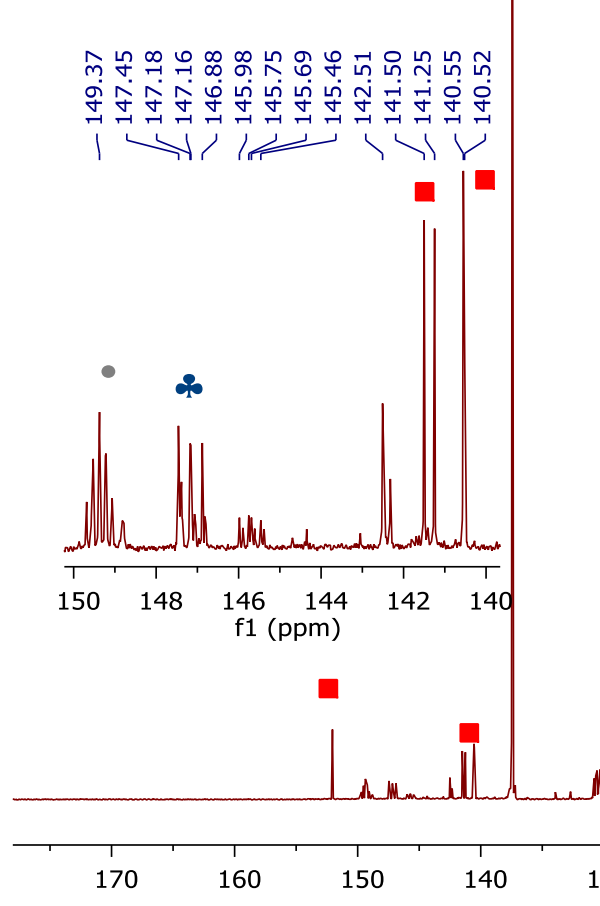

$160 \quad 150$

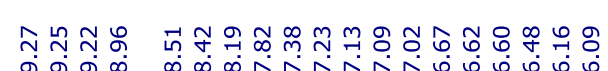

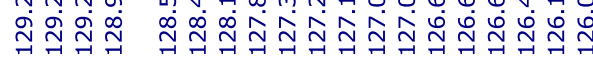

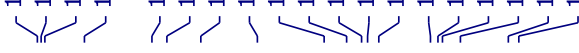

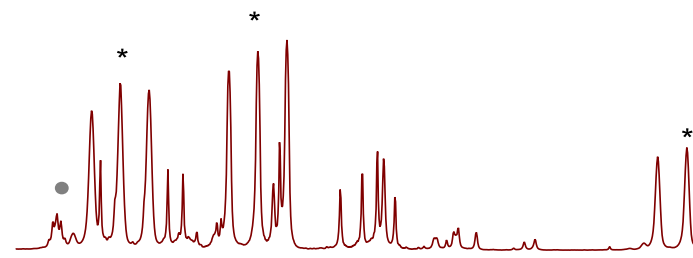

129.5129 .0128 .5128 .0127 .5127 .0126 .5126 .0125 .5125 .0 f1 1 (ppm)
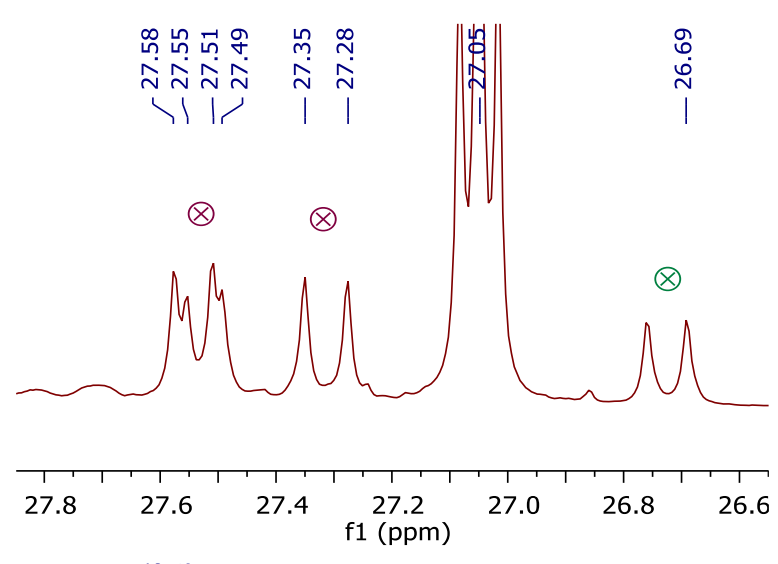

(n)

* Tol- $d_{n}$

- Complex $\left[\left(\mathrm{L}_{1}\right)_{2} \mathrm{Ni}\right](7)$

3. Complex $\left[\left(\mathrm{L}_{1}\right) \mathrm{Ni}(\mathrm{cod})\right](8)$

$\otimes$ Complex $\left[\left(\mathbf{L}_{1}\right) \mathrm{Ni}(\mathbf{3 a a})\right]$ (11-major)

$\otimes$ Complex $\left[\left(\mathrm{L}_{1}\right) \mathrm{Ni}(3 \mathbf{a a})\right]$ (11-minor)

- $3 \mathbf{a} a$

11

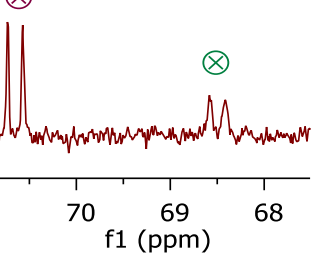

in in in$$
\mathrm{f} 1 \text { (ppm) }
$$
$70 \begin{gathered}69 \\ \mathrm{f} 1(\mathrm{ppm})\end{gathered}$ 68
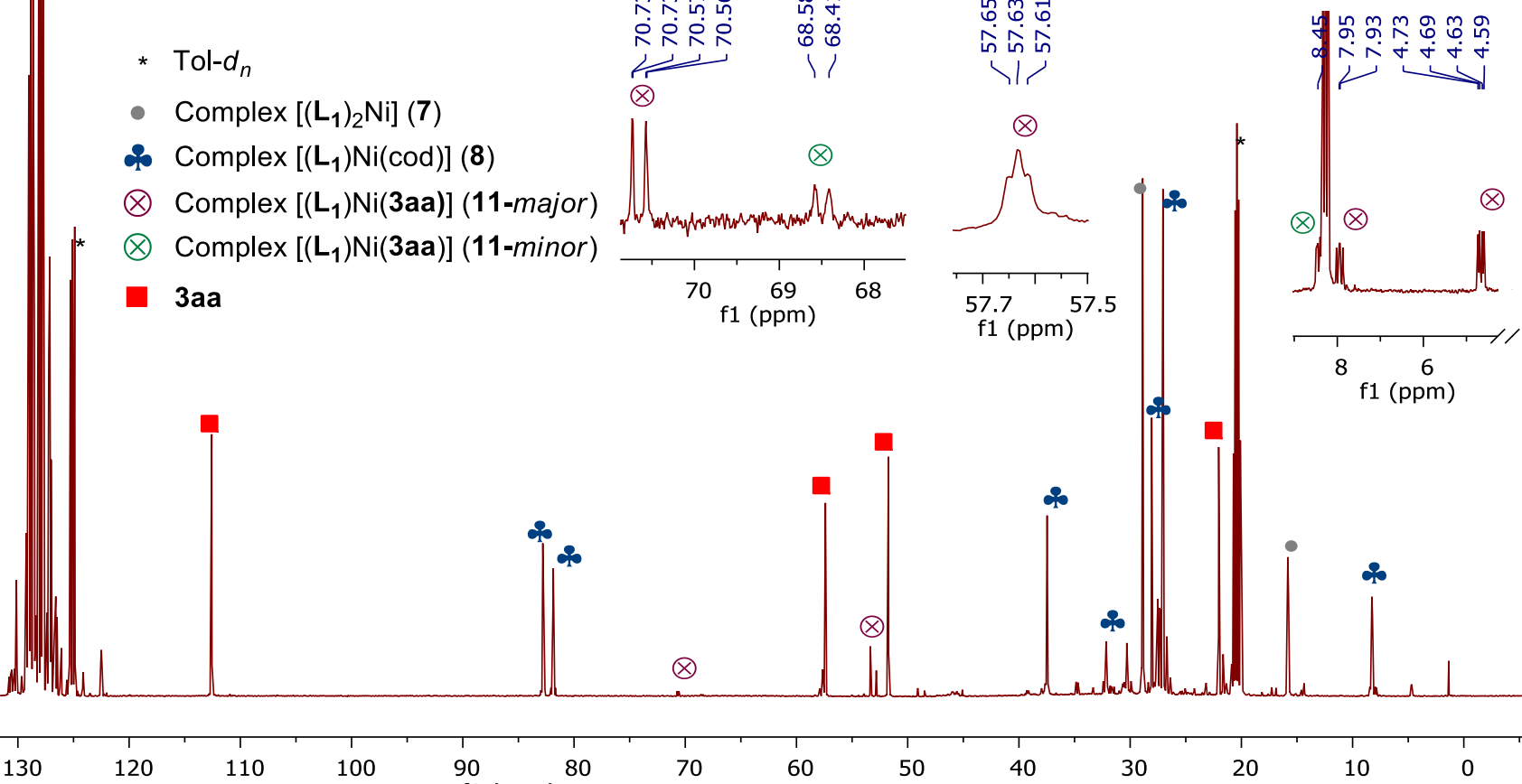

120

110

100

$90{ }^{8} 1(\mathrm{ppm})^{80}$

$\otimes$ 
$\mathrm{Me}$

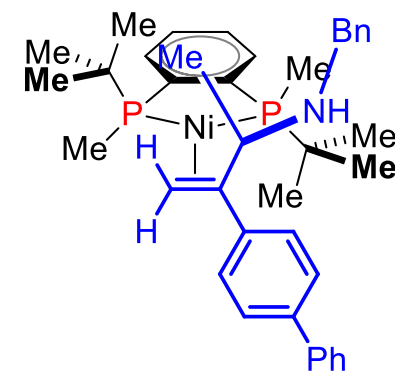

[( $\left.\left.\mathrm{L}_{1}\right) \mathrm{Ni}(\mathbf{3 a a})\right]$ (11-major)

${ }^{13} \mathrm{C}\left\{{ }^{1} \mathrm{H}\right\}$ NMR (130 MHz, Toluene- $\left.d_{8}\right)$

- Complex $\left[\left(\mathrm{L}_{1}\right)_{2} \mathrm{Ni}\right](7)$

a. Complex $\left[\left(\mathrm{L}_{1}\right) \mathrm{Ni}(\mathrm{cod})\right](8)$

$\otimes$ Complex $\left[\left(\mathrm{L}_{1}\right) \mathrm{Ni}(\mathbf{3 a a})\right]$ (11-major $)$

$\otimes$ Complex $\left[\left(\mathrm{L}_{1}\right) \mathrm{Ni}(\mathbf{3 a a})\right]$ (11-minor $)$

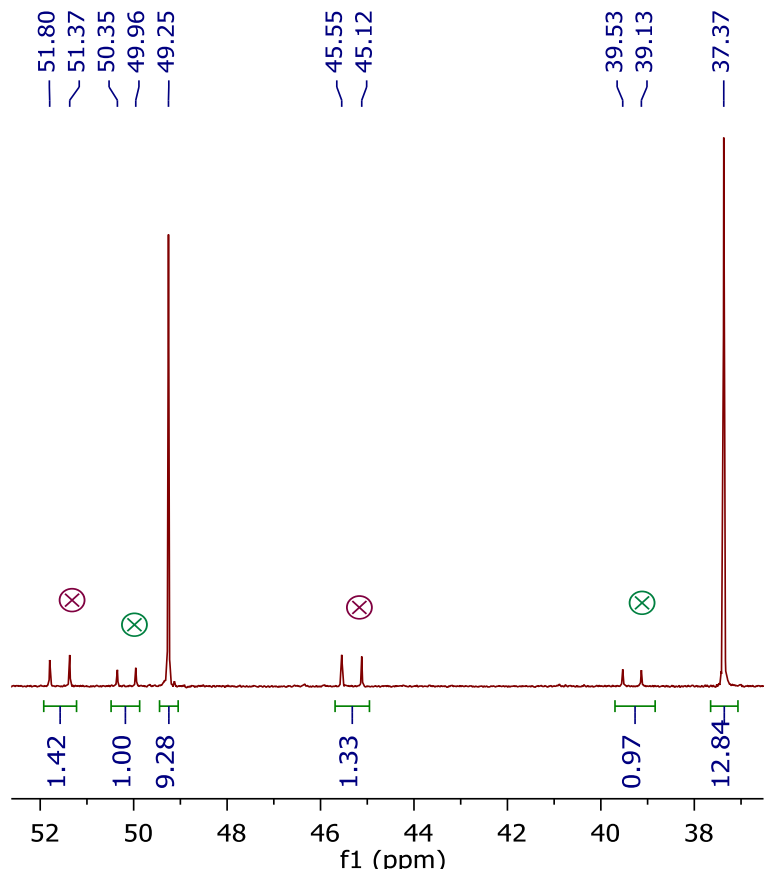

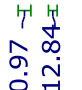

$35 \quad 30$

$25 \quad 20$

$15 \quad 10$

0

$\begin{array}{lllllll}-10 & -15 & -20 & -25 & -30 & -35 & -40\end{array}$ 


\section{X-Ray analysis}

All data were collected on an Agilent Supernova diffractometer equipped with an ATLAS CCD detector using Cu radiation. The crystal was kept at 180.01(10) K during data collection. Using Olex2, the structure was solved with the ShelXT structure solution program using Intrinsic Phasing and refined with the ShelXL refinement package using Least Squares minimization. ${ }^{5}$ Crystallographic data are found in Table S3. 
Identification code

CCDC

Empirical formula

Formula weight

Temperature/K

Crystal system

Space group

a/Å

$\mathrm{b} / \AA$

$\mathrm{c} / \AA$

$\alpha /^{\circ}$

$\beta /^{\circ}$

$\mathrm{y} /{ }^{\circ}$

Volume/Å3

Z

$\rho_{\text {calc }} \mathrm{g} / \mathrm{cm}^{3}$

$\mu / \mathrm{mm}^{-1}$

$\mathrm{F}(000)$

Crystal size $/ \mathrm{mm}^{3}$

Radiation
$3 a a$

1938704

$\mathrm{C}_{23} \mathrm{H}_{24} \mathrm{CIN}$

349.88

180.01(10)

monoclinic

$\mathrm{P} 2{ }_{1}$

10.48643(15)

7.50634(11)

12.56284(19)

90

91.0457(13)

90

988.72(2)

2

1.175

1.719

372.0

$0.957 \times 0.155 \times 0.076$

CuKa $(\lambda=1.54184)$

$2 \Theta$ range for data collection $/{ }^{\circ} 7.038$ to 141.102

Index ranges

Reflections collected

Independent reflections

Data/restraints/parameters

Goodness-of-fit on $\mathrm{F}^{2}$

Final $R$ indexes $[I>=2 \sigma(I)]$

Final $\mathrm{R}$ indexes [all data]

Largest diff. peak/hole / e $\AA^{-3}$ 0.13/-0.17

Flack parameter

$-0.012(13)$
$-12 \leq \mathrm{h} \leq 12,-9 \leq \mathrm{k} \leq 9,-15 \leq \mathrm{I} \leq 15$

15843

$3737\left[R_{\text {int }}=0.0315, R_{\text {sigma }}=0.0208\right]$

$3737 / 1 / 236$

1.033

$\mathrm{R}_{1}=0.0278, \mathrm{wR}_{2}=0.0705$

$\mathrm{R}_{1}=0.0298, \mathrm{wR}_{2}=0.0718$

Table S3. Crystal structure and data refinement for 3aa-HCl salt (CCDC 1938704) 


\section{References}

${ }^{1}$ Fiorito, D.; Folliet, S.; Liu, Y.; Mazet, C. A General Nickel-Catalyzed Kumada Vinylation for the Preparation of 2-Substituted 1,3-Dienes ACS Catal. 2018, 8, 1392.

${ }^{2}$ Doi, H. ; Sakai, T. ; Yamada, K.-I. ; Tomioka, K. N-Allyl-N-tert-butyldimethylsilylamine for chiral ligand-controlled asymmetric conjugate addition to tert-butyl alkenoates. Chem. Commun. 2004, 1850.

${ }^{3}$ Burés, J. Variable Time Normalization Analysis: General Graphical Elucidation of Reaction Orders from Concentration Profiles. Angew. Chem. Int. Ed. 2016, 55, 16084-16087.

${ }^{4}$ (a) http://supramolecular.org (b) Thordarson, P. Determining Association Constants from Titration Experiments in Supramolecular Chemistry. Chem. Soc. Rev. 2011, 40, 1305. (c) Hibbert, D. B.; Thordarson, P. The Death of the Job Plot, Transparency, Open Science and Online Tools, Uncertainty Estimation Methods and Other Developments in Supramolecular Chemistry Data Analysis. Chem. Commun. 2016, 52, 12792. (d) Fit for 1:1 model: http://app.supramolecular.org/bindfit/view/4d262417-28b2-4c9b-8a4d-3a9f4f48be85. (e) Fit for 1:2 model: http://app.supramolecular.org/bindfit/view/65f19228-fb6e-48a1-9bd1d8f2f5b5344f. (f) Fit for 2:1 model: http://app.supramolecular.org/bindfit/view/d1bb8d30-cf614fe6-afe9-b8adbe0af3e8.

${ }^{5}$ (a) Dolomanov, O. V.; Bourhis, L. J.; Gildea, R. J; Howard, J.A.K.; Puschmann, H. OLEX2: A Complete Structure Solution, Refinement and Analysis Program. J. Appl. Cryst. 2009, 42, 339. (b) Sheldrick, G. M. SHELXT - Integrated Space-Group and Crystal-Structure Determination. Acta Cryst. 2015, A71, 3. (c) Sheldrick, G. M. Crystal Structure Refinement with SHELXL. Acta Cryst. 2015, C71, 3. 